\title{
THE CHARACTERIZATION OF TA-289, A NOVEL ANTIFUNGAL FROM Fusarium $s p$.
}

Natelle C. H. Quek

A thesis submitted to Victoria University of Wellington in part fulfilment of the requirements for the degree of Master of Science in Biotechnology

School of Biological Sciences

Victoria University of Wellington, New Zealand 2011 
For Dad, my pillar,

Mi, my heart,

and Jie, my best friend. 


\section{ABSTRACT}

Natural products offer vast structural and chemical diversity highly sought after in drug discovery research. Saccharomyces cerevisiae makes an ideal model eukaryotic organism for drug mode-of-action studies owing to ease of growth, sophistication of genetic tools and overall homology to higher eukaryotes. Equisetin and a closely related novel natural product, TA-289, are cytotoxic to fermenting yeast, but seemingly less so when yeast actively respire. Cell cycle analyses by flow cytometry revealed a cell cycle block at $\mathrm{S}-\mathrm{G}_{2} / \mathrm{M}$ phase caused by TA-289; previously described oxidative stress-inducing compounds causing cell cycle delay led to further investigation in the involvement of equisetin and TA289 in mitochondrial-mediated generation of reactive oxygen species. Chemical genomic profiling involving genome-wide scans of yeast deletion mutant strains for TA-289 sensitivity revealed sensitization of genes involved in the mitochondria, DNA damage repair and oxidative stress responses, consistent with a possible mechanism-of-action at the mitochondrion. Flow cytometric detection of reactive oxygen species (ROS) generation caused by TA-289 suggests that the compound may induce cell death via ROS production. The generation of a mutant strain resistant to TA-289 also displayed resistance to a known oxidant, $\mathrm{H}_{2} \mathrm{O}_{2}$, at concentrations that were cytotoxic to wild-type cells. The resistant mutant displayed a higher basal level of ROS production compared to the wild-type parent, indicating that the resistance mutation led to an up-regulation of antioxidant capacity which provides cell survival in the presence of TA-289. Yeast mitochondrial morphology was visualized by confocal light microscopy, where it was observed that cells treated with TA-289 displayed abnormal mitochondria phenotypes, further indicating that the compound is acting primarily at the mitochondrion. Similar effects observed with equisetin treatment suggest that both compounds share the same mechanism, eliciting cell death via ROS production in the mitochondrial respiratory chain. 


\section{ACKNOWLEDGEMENTS}

And this is the part where I get to exclaim in the most stupendous fashion, "Made it, Ma! Top of the world!' (Seriously, you get the best quotes from 1950s gangster films. That, and Gilmore Girls - nothing gets better than, "May Destiny's Child break up if I count these blueberries"). But I digress, as usual.

I can write a short novel, in haiku, while standing on my head, about all the people that made it worth while, but unfortunately, the wittiness ends here. And also because, I know I don't have to make their names rhyme with an amusing inanimate object for them to understand what I'm trying to say; My most heartfelt gratitude to those who gave this silly little girl a chance, with whom I shared everything, whom I could always count on, who made it all possible, of whom I greatly admired, and who has never let me fall and will never let me fall, In This Order: Dr David Bellows, Veronica Venturi (withhair-so-curly, and takes-her-cappuccino-extra-foamy)(I couldn't resist, it wasn't that horrible was it? Cappuccinos are pretty amusing inanimate objects...), Christina Roberts, Professor Paul-grandpa-Atkinson, Dr David Maass, and Bear.

To the greater Chemical Genetics family, where much laughter of the guttural kind (which really is the best kind of laughter) were thrown and heaved; special mentions go to Darryl tangata-whenua Jones, Yee Suen my-body-mass-index-is-zero! Low, Namal I'm-comfortable-with-my-sexuality Coorey, Hannah pink-bits Hoang, and Peter littleblack-bunny Bircham. To Dr David Burton, Dr David Ackerley, Dr Jim McIntosh, and Professor John I-need-Buddhist-prayer-flags-to-help-me-get-grants Miller, whose enthusiasms in their teachings made me realise as a wayward undergraduate that Science (see, I capitalised it. Now it's a proper noun) really is actually kind of cool.

Most importantly, I would not be here, and I would not wish to be anywhere else, if I didn't have my Dad, my Mi, and my Jie. Your support and love is overwhelming (but please, don't stop!), and I am the luckiest and most proud to have you as my family. This is for you. 


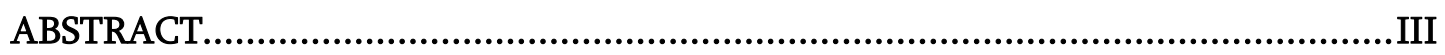

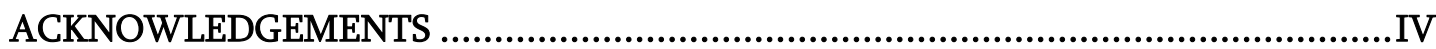

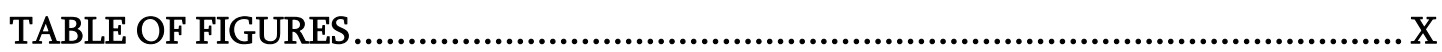

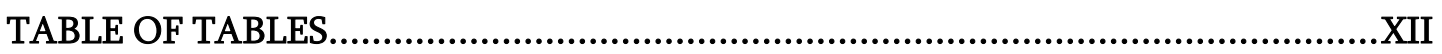

LIST OF ABBREVIATIONS ............................................................................. XIII

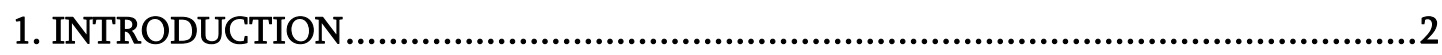

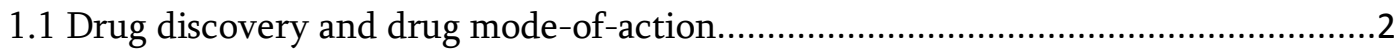

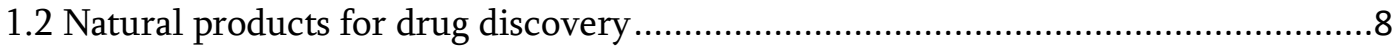

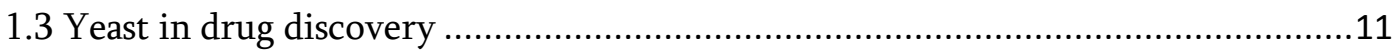

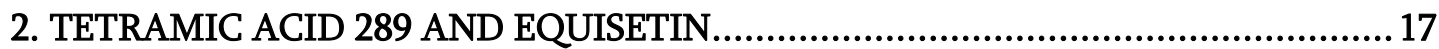

2.1 Introduction: Natural products from terrestrial microbes.....................................17

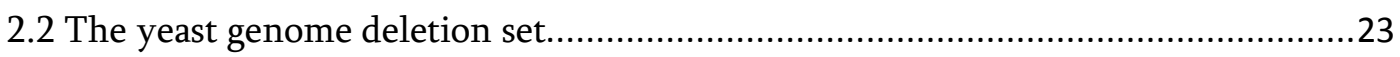

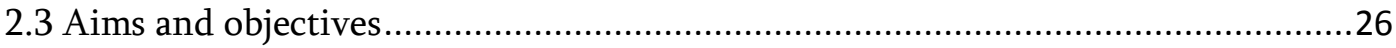

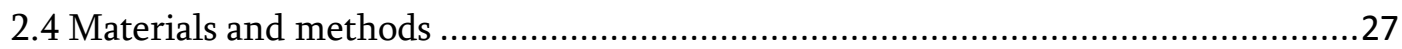

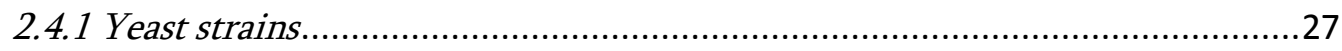

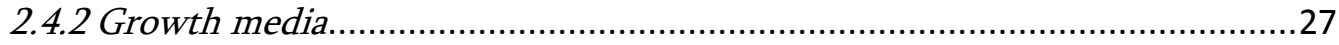

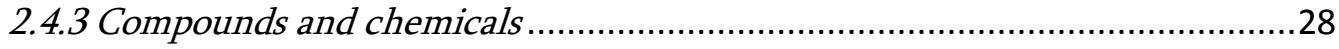

2.4.4 Growth inhibition in liquid media ............................................................29

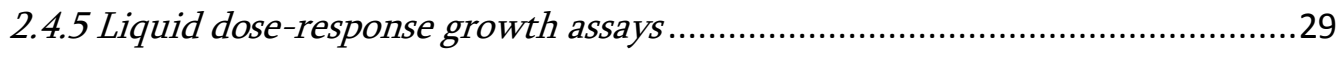

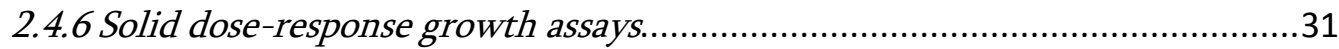

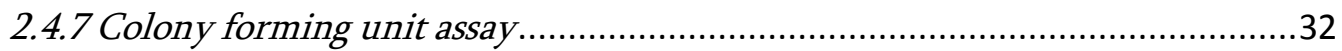

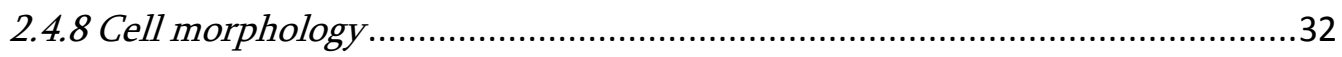

2.4.9 Cell cycle analysis by flow cytometry …....................................................33

2.4.10 Determining the presence of cell populations by flow cytometry .................34

2.4.11 Haplo-insufficiency and homozygous profiling.........................................35

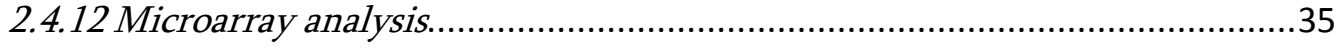

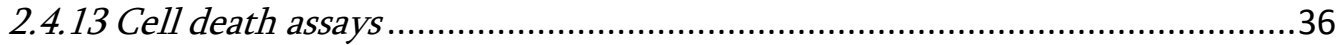

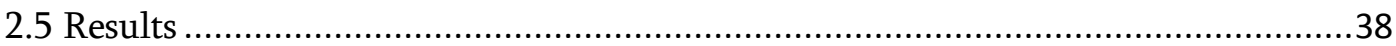


2.5.1 Initial screening of natural product isolates ....

2.5.2 TA-289 and equisetin display pH-dependent cytotoxic activities..................39

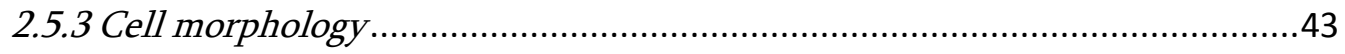

2.5.4 TA-289 and equisetin cause late phase cell cycle block...............................47

2.5.5 Chemical genomic profiling of TA-289.................................................51

2.5.6 TA-289 and equisetin: Glucose vs. Glycerol as a carbon source .....................59

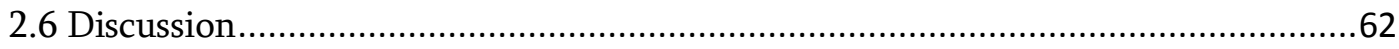

2.6.1 Biologically active compounds from terrestrial fungi...................................62

2.6.2 Effects of TA-289 and equisetin on cell morphology and cell cycle...............64

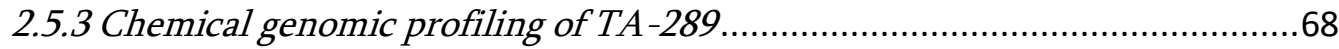

2.5.4 Glucose vs. Glycerol as a carbon source …................................................72

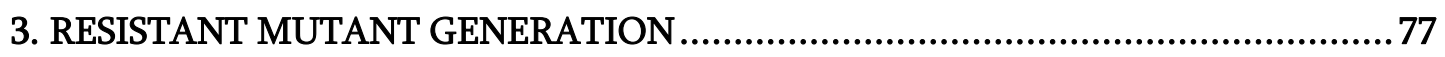

3.1 Introduction: Resistant mutant generation …......................................................77

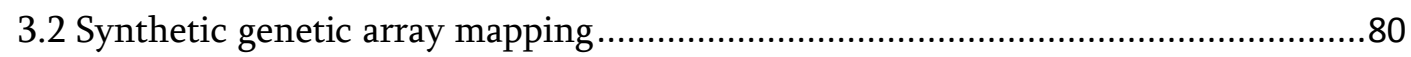

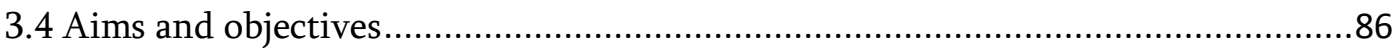

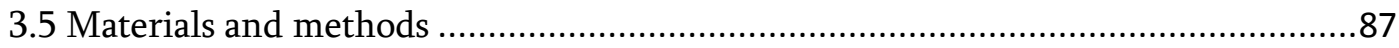

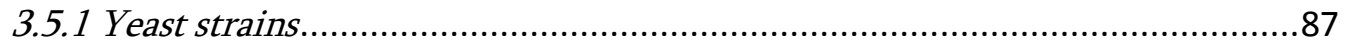

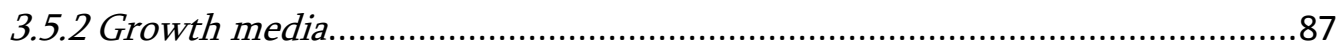

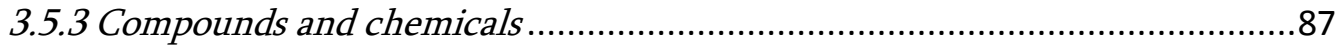

3.5.4 Resistant mutant generation by ethane methyl sulfonate mutagenesis..........88

3.5.5 Confirmation of resistance by serial spot dilution ........................................89

3.5.6 Identifying mutant phenotypes under differing stress conditions.................90

3.5.7 Creating an isogenic wild-type for mating with resistant mutants ................91

3.5.7.1 PCR amplification of the URA3 deletion cassette .................................91

3.5.7.2 High efficiency transformation with URA3 deletion cassette................92

3.5.7.3 Confirmation of transformants by colony PCR ....................................93

3.5.8 Determining dominance and/or recessiveness of resistant mutants ...............94

3.5.8.1 Generating heterozygous diploid mutants..........................................94

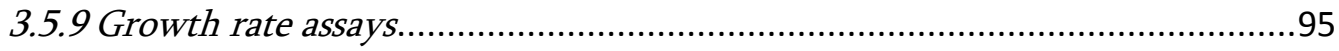

3.5.10 Proof-of-principle C-SGAM with microarray: Using cycloheximide as a pilot 
3.5.11 Microarray analysis.

3.5.12 Reactive oxygen species detection by flow cytometry ................................98

3.5.13 Cell death assays ...............................................................................99

3.5.14 C-SGAM of TA-289 resistant mutant by hydrogen peroxide .....................100

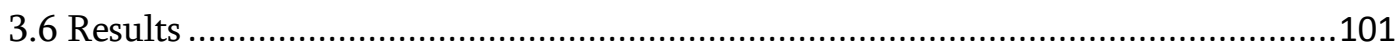

3.6.1 Resistant mutant generation.................................................................101

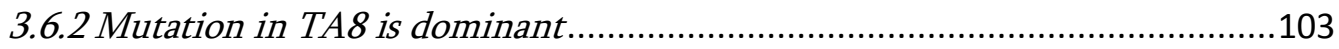

3.6.3 Proof-of-principle C-SGAM with microarray ............................................104

3.6.4 TA8 mutant displays resistance by upregulating anti-oxidant production...106

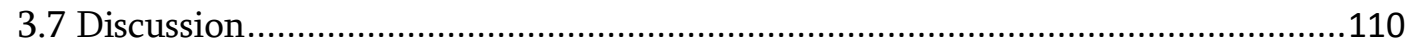

3.7.1 Resistant mutant generation.................................................................110

3.7.2 Mapping the resistance-conferring mutation .........................................112

4. REACTIVE OXYGEN SPECIES IN Saccharomyces cerevisiae .............................. 119

4.1 The role of mitochondria in the production of reactive oxygen species ..............119

4.2 Mitochondrial ROS production and yeast apoptosis ..........................................122

4.3 Inhibitors of the electron transport chain and the generation of reactive oxygen

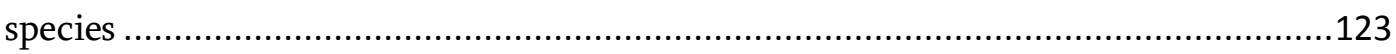

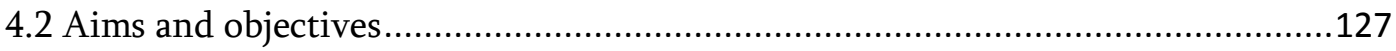

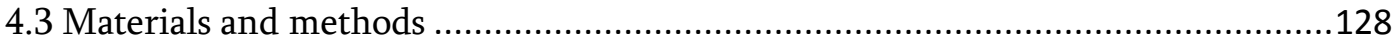

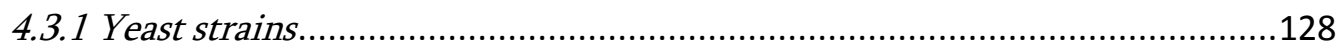

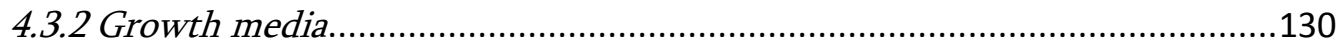

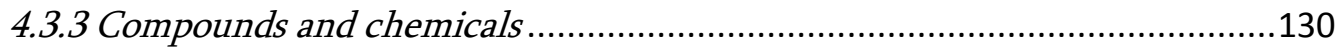

4.3.4 Detecting reactive oxygen species by flow cytometry...............................131

4.3.5 Detecting mitochondrial activity by flow cytometry ................................131

4.3.6 High-throughput visualization of mitochondria ........................................132

4.3.7 Liquid dose-response assays..................................................................132

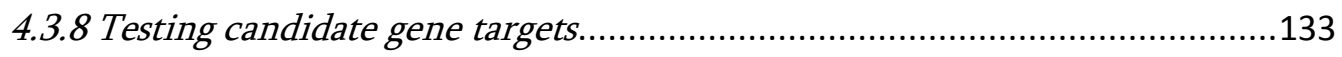

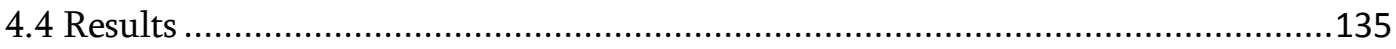

4.4.1 TA-289 causes the production of reactive oxygen species..........................135

4.4.2 TA-289 and equisetin effect mitochondria and alter mitochondrial morphology ….........................................................................................

4.4.3 High salt does not sequester potency of TA-289 and equisetin..................142 
4.4.4 Testing candidate genes: Non-ETC genes

4.4.5 Testing candidate genes: ETC genes ...........................................................146

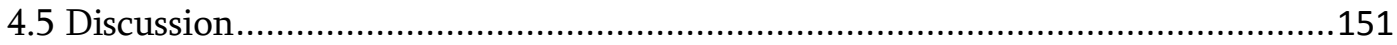

4.5.1 Effects of TA-289 and equisetin on the redox state of yeast........................151

4.5.2 The effects of TA-289 and equisetin on yeast mitochondria ........................154

4.5.3 Potential molecular targets of TA-289 and equisetin: The ETC ..................157

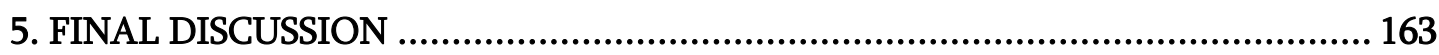

5.1 Mitochondrial-mediated ROS production in yeast ...............................................163

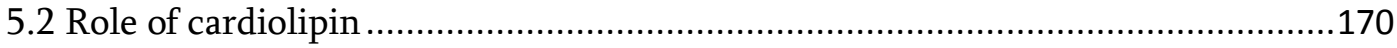

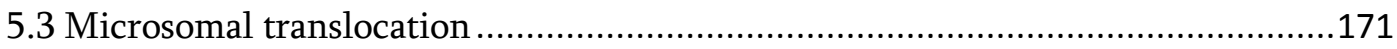

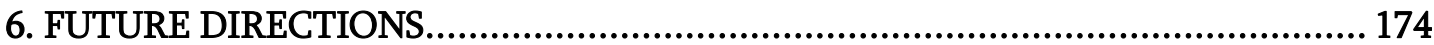

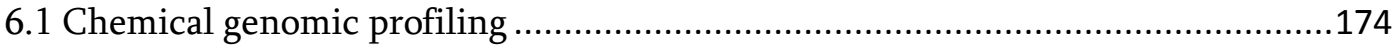

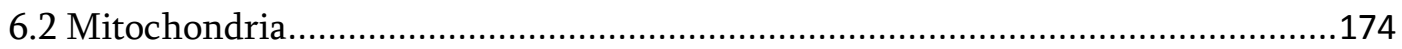

6.2.1. Mitochondrial transmembrane potential..................................................174

6.2.2. Mitochondrial deficient mutants............................................................175

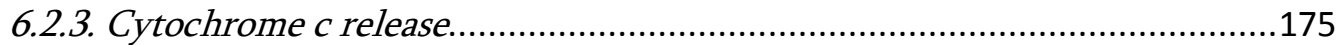

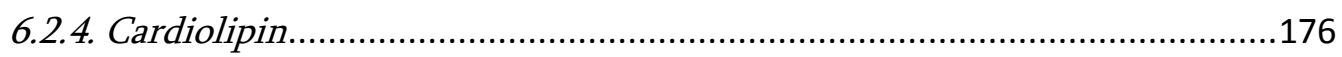

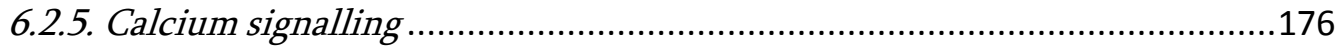

6.3 The effect of fermentable and non-fermentable carbon sources .........................177

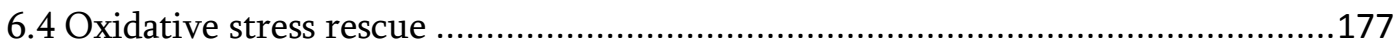

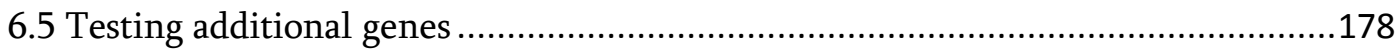

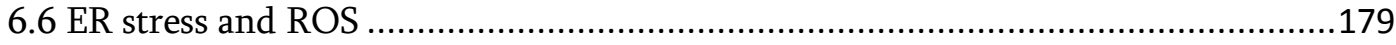

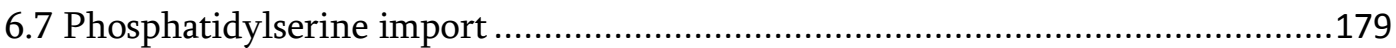

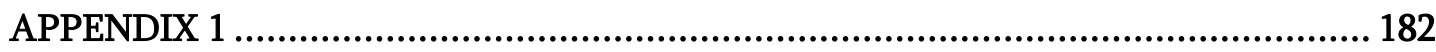

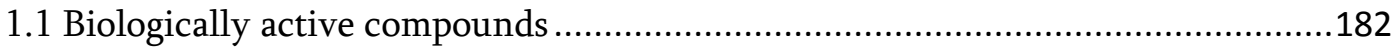

1.2 Liquid-dose response assays of TA-289 and equisetin in varying $\mathrm{pH}$ conditions ..185

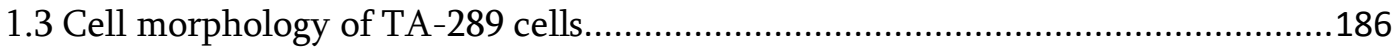

1.4 Cell cycle analysis by flow cytometry: Time-course histograms ........................187

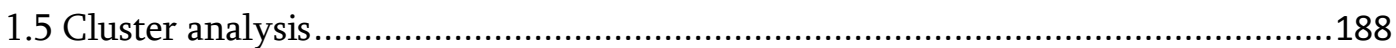


1.6 TA-289 HOM screen overlaps with L-Dopa and MPP+ 189

1.7 Cell death assays: Glucose vs. Glycerol as a carbon source....................................196

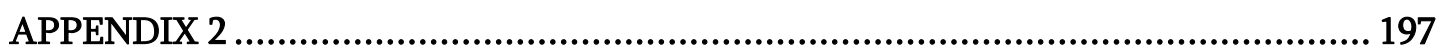

2.1 Resistant mutants and cross-resistance scores ...................................................197

2.2 Generating an isogenic MATa wild-type strain ...............................................199

2.2.1 Isogenic wild-type creation and confirmation ..........................................199

2.2.2 Making heterozygous diploid mutants .....................................................200

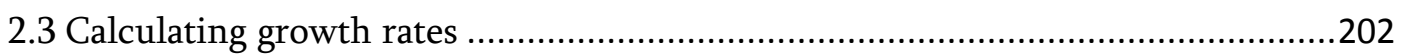

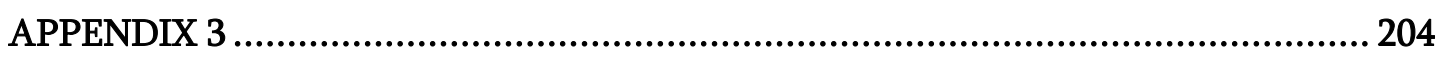

3.1 High salt does not sequester potency of TA-289 and equisetin...........................204

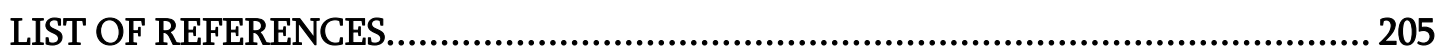




\section{TABLE OF FIGURES}

Figure 1.1. All small molecule new entities launched between 1981-2006 10

Figure 1.2. Structural diversity and biological specificity of NPs 11

$\begin{array}{lr}\text { Figure 2.1. Structures of TA-289 and equisetin } & 19\end{array}$

Figure 2.2. Chemical structures of structurally-related tetramic acid compounds 22

Figure 2.3. Bioactive natural product isolates display differential activity depending on the PDR state of the yeast cell

Figure 2.4. Growth-inhibitory effects of TA-289 are $\mathrm{pH}$-dependent

Figure 2.5. Solid dose-response assay of TA-289 and equisetin

Figure 2.6. TA-289 and equisetin are cytotoxic to $p d r \Delta$ yeast cells

Figure 2.7. TA-289 causes a small-size phenotype in yeast

Figure 2.8. Cells are present after $18 \mathrm{~h}$ treatment with TA-289 or equisetin

Figure 2.9. TA-289 and equisetin cause late-phase cell cycle block

Figure 2.10. TA-289 causes irreversible cell cycle block in $p d r \Delta$ cells

Figure 2.11. TA-289 and equisetin do not target tubulin

Figure 2.12. Close up clustergram of both TA-289 HOM screens 56

Figure 2.13. Gene localizations of HOM screen compared to whole genome

Figure 2.14. TA-289 kills pdr $\Delta$ cells within $2 \mathrm{~h}$ of treatment when glucose is used as a carbon source

Figure 2.15. TA-289 does not kill when glycerol-ethanol is used as a carbon source

Figure 3.1. A simplified flow diagram of the C-SGAM methodology 
Figure 3.3. TA-289 and equisetin resistant mutants

Figure 3.4. Mutation in TA8 is dominant

Figure 3.5. Linkage disequilibrium pattern around RPL28 locus

Figure 3.6. A close-up view of neighbouring genes to RPL28.

Figure 3.7. TA8 mutant displays a higher basal level of ROS production

Figure 3.8. TA8 is resistant to $\mathrm{H}_{2} \mathrm{O}_{2}$

Figure 3.9. TA8 is more sensitive to $\mathrm{H}_{2} \mathrm{O}_{2}$ under respiratory conditions

Figure 3.10. Parallel analysis by DNA microarray for resistance mapping

Figure 4.1. A simplified model of the mitochondrial ETC and ROS production

Figure 4.2. A simplified model of the Q-cycle at complex III and the mechanism of superoxide production

Figure 4.3. TA-289 causes production of ROS

Figure 4.4. TA-289 and equisetin have an affect on mitochondria

Figure 4.5.A. Cir2p-GFP Untreated control cells

Figure 4.5.B. Cir2p-GFP Cells treated with $50 \mu \mathrm{M}$ TA-289

Figure 4.5.C. Cir2p-GFP Cells treated with $50 \mu \mathrm{M}$ equisetin

Figure 4.6. Sensitivity of non-ETC genes to TA-289

Figure 4.7. Sensitivity of complex I and complex II genes to equisetin

Figure 4.8. Sensitivity of complex III genes to equisetin 


\section{TABLE OF TABLES}

Table 2.1. MIC values of TA-289 and equisetin in wt and $p d r \Delta$ strains under different media conditions

Table 2.2. Cell sizes of wt and $p d r \Delta$ cells treated with TA-289

Table 2.3. Percentage of both wt and $p d r \Delta$ cells in $\mathrm{G}_{1}, \mathrm{~S}$, and $\mathrm{G}_{2} / \mathrm{M}$ phase

Table 2.4. List of top 40 hypersensitive hits from HOM screens

Table 2.5. List of top 15 hypersensitive hits from the HET screen

Table 2.6. MIC values of TA-289 and equisetin in wt and $p d r \Delta$ strains under different carbon sources

Table 3.1. Table describing resistant mutants that display differential sensitivity under varying stress conditions

102

Table 4.1. Table showing percentage of live, ROS, ROS and dying, and propidium iodidepermeable cell populations

136

Table 4.2. MIC values of TA-289 and equisetin in wt and $p d r \Delta$ strains in the presence or absence of $\mathrm{NaCl}$ 


\section{LIST OF ABBREVIATIONS}

$\Delta \Psi \mathrm{m}$

ADP

ATP

bp

C-SGAM

Can

$\mathrm{CYH}$

clonNAT/NAT

DCF

DMA

DMSO

DTT

EMS

EQ

G418

GFP

HET

HIP

$\mathrm{HOM}$

IMM

kanR

natR

$\mathrm{NADH}$

ND

NP mitochondrial transmembrane potential

adenosine diphosphate

adenosine triphosphate

base pair

chemical-synthetic genetic array mapping

canavanine

cycloheximide

nourseothricin

2,7-dichlorofluorescein diacetate

deletion mutant array

dimethyl sulfoxide

dithiothreitol

ethane methyl sulfonate

equisetin

geneticin

green fluorescent protein

heterozygous deletion set

haplo-insufficiency profiling

homozygous deletion set

inner mitochondrial membrane

kanamycin resistance cassette

nourseothricin resistance cassette

reduced nicotinamide adenine dinucleotide

natural product-derived

natural product 


\begin{tabular}{|c|c|}
\hline OD & optical density \\
\hline ORF & open reading frame \\
\hline PCR & polymerase chain reaction \\
\hline PCD & programmed cell death \\
\hline PDR & pleiotropic drug resistance \\
\hline PEG & polyethylene glycol \\
\hline ROS & reactive oxygen species \\
\hline SC & synthetic complete \\
\hline $\mathrm{SD}$ & synthetic drop-out \\
\hline SDS & sodium dodecyl sulfate \\
\hline SGA & synthetic genetic array \\
\hline SGAM & synthetic genetic array mapping \\
\hline SL & synthetic lethality \\
\hline TA & tetramic acid \\
\hline TAP-tag & tandem affinity purification tag \\
\hline Thia & thialysine \\
\hline wt & wild-type \\
\hline YPD & yeast peptone dextrose \\
\hline
\end{tabular}




\section{INTRODUCTION}

\subsection{Drug discovery and drug mode-of-action}

Drug discovery research is an interdisciplinary endeavour with strong foundations in both biology and chemistry. Essential foundations of chemical theory strongly guided by pharmacology, biochemistry, and molecular biology saw a marked increase in the contribution of drug discovery to the progress of medicine in the last century (Laport, Santos et al. 2009). Advances in synthetic chemistry, especially diversity-oriented synthesis for production of natural product-like small molecule inhibitors in high-throughput (Schreiber 2000; Kumar, Kiuchi et al. 2005), and natural product isolation, coupled with improved technology available for structural elucidation has allowed the characterization of large libraries of distinct chemical entities with diverse skeletal structure. Biological assay development in combination with high-throughput screening technology (Bradner, McPherson et al. 2006) has enabled identification of numerous compounds with diverse biological activity, a significant proportion of which possess therapeutic properties.

As modern medicine becomes increasingly dependent on drug-like compounds, the continuous discovery and development of new small molecule inhibitors for drugleads is crucial. As such, the discovery of novel compounds and especially compounds with novel biological activity greatly expands the pharmaceutical arsenal with which to combat disease, in particular, disease areas dearth in 
competent drugs, or where current therapeutics are less effective now compared to when they were first applied. This is exemplified in the case of anti-bacterial, antiviral, anti-fungal, and anti-parasitic drugs that are prone to decreased or complete loss of efficacy as a result of natural evolutionary resistance (White, Marr et al. 1998; Walsh 2000). Penicillin, for example has been widely prescribed as an antibiotic, however growing resistance to penicillin has fuelled research into more potent analogues such as ampicillin, as well as the discovery of mechanistically similar antibiotics, like the cephalosporins (Wheeler 1977), and equally, mechanistically dissimilar antibiotics, such as the tetracyclines (Duggar 1948). In the case of cancer therapeutics, acquisition of resistance to anti-cancer drugs by cancer cells is a major problem, where the mechanism of resistance to anti-cancer agents appears to be multi-factorial, and varies greatly depending on the type of cancer (Gottesman 2002; Okuma, Kiguchi et al. 2003; Roos, Jost et al. 2011). For the drug discovery process, studies into mechanisms of resistance aid in the discovery of new anti-cancer drugs with differing mechanisms that are able to overcome resistance by cancer cells.

Drug target selectivity is an important factor when considering a drug-like molecule for development in the drug discovery process. While greater target selectivity often means lesser side-effects, this is not always true. Dominating the cancer therapeutic setting are anti-microtubule drugs (Morris and Fornier 2008), drugs that arrest mitosis and cause cell death. Although these drugs have been proven useful in 
targeting rapidly dividing cancer cells, non-tumorigenic cells that are actively dividing within the host, such as epithelial and hair follicle cells are also targeted (Botchkarev 2003). Therefore, there are several important factors when considering a drug-like molecule, especially one that is deserving of clinical follow-up. Drug discovery research has seen the evolution of the nature in which compounds are screen and selected for development as the need for drugs with greater efficacy, better target selectivity, and favourable pharmacokinetic properties is becoming increasingly apparent. Assays with greater sensitivity in identifying drug-target activity and specificity have become a necessity, and where possible, biological assays that mimicked settings in vivo are applied.

Molecular biology has offered drug discovery a better understanding of disease processes and the dissection of potential targets and biological pathways, especially at the genomic level (Drews 2000). As more structurally and chemically diverse small molecules are discovered, there has been a considerable effort made towards applying these small molecules to dissect cellular pathways in a manner analogous to that applied in genetics. Small molecule inhibitors or activators are employed to produce phenotypes of interest, after which the target responsible for the induced phenotype is characterized. This approach is largely beneficial for the drug discovery process, as it simultaneously identifies proteins or cellular pathways that can be potential targets for therapeutic intervention, as well as small molecules that alter 
the functions of these targets (Schreiber 2000). For instance, rapamycin, inhibitor of the target of rapamycin (TOR) proteins (Heitman, Movva et al. 1991), induces starvation responses in both yeast and mammalian cells (Singh, Sun et al. 1979). Overexpression studies in yeast revealed additional genes associated with cellular pathways of TOR, wherein these genes also display sensitivity to LY-83583, a small molecule suppressor of rapamycin-induced inhibition (Butcher, Bhullar et al. 2006). Through these studies, functional links between genes implicated in the TOR pathway affecting sensitivity to both rapamycin and LY-83583 facilitated the investigation of potential cellular targets of LY-83583, in addition to suggesting its mechanisms.

The move from drug development programs predominantly screening purified enzymes and receptors in vitro (Sturgeon, Kemmer et al. 2006), to complex cellbased phenotypic screens containing a multitude of potential targets has lead to greater emphasis on drug mode-of-action research. The development of cell-based assays therefore plays a pivotal role in targeted drug discovery, as compounds are screened for activity wherein target proteins are already in their native environment. In cell-based drug screens, the observed biological activity could be due to interactions with numerous potential targets, and so drug mode-of-action studies are often required to understand the basis of the activity of the drug, 
especially in the case where this results in its advancement as a therapeutic drug in any disease setting.

Drug target or mode-of-action identification is essential in the drug discovery process, especially in the case where side effects are concerned. Side effects often occur as a result of a drug having multiple targets, as it was in the case of the nonsteroidal anti-inflammatory drugs, salicylate and aspirin. While both drugs exert their anti-inflammatory effects through inhibition of cyclooxygenases (Vane 1994), they also have been shown to target multiple connected cellular pathways involved in the pathogenesis of the inflammatory response such as the NF- $\mathrm{B}$ (nuclear factor kappa B) pathway (Kopp and Ghosh 1994). The contribution of the potential targets to the mode-of-action of these drugs still remain undetermined (Yin, Yamamoto et al. 1998; Alpert and Vilcek 2000). Side effects also arise from a target-mediated effect, where the target(s) plays different roles in various cell types, such as the case of the immunosuppressant cyclosporine and its cellular targets, the cyclophilins. Cyclosporine interacts with cyclophilin A, where the resulting complex prevents production of pro-inflammatory molecules TNF- $\alpha$ and interleukin 2 (Serkova 2004). However, cyclosporine also interacts with cyclophilin D, a key componenet in the permeability transition pores within the mitochondrial matrix, the disruption of which results in mitochondrial swelling and dysfunction (Elrod, Wong et al. 2010). Drug target identification strategies allow for the distinction of promiscuous drugs, 
as in the first example, from pleiotropic targets. In the latter case, it can reveal undesirable disease targets, and for the former, equally undesirable compounds for therapeutic use.

Conversely, for non-therapeutic compounds, a well-defined mode-of-action may allow for their applications as biological probes, used to explore the functions and roles of their targets in cellular pathways and processes. For example, the protein synthesis inhibitor cycloheximide has been extensively used to assess the contribution of protein synthesis to a given biological phenomenon (Maia 1994; Funder 2005). Alternatively, the compound could be used to identify unknown functions of its target. The highly conserved heat shock protein 90 (HSP90) chaperone complex, for example, which is involved in steroid receptor function and the stability of oncogenic kinases is a known target for the natural product (NP) compound geldanamycin (Cardenas, Cruz et al. 1999). Genome-wide studies in the yeast Saccharomyces cerevisiae using geldanamycin as a biochemical probe allowed for the mapping of the genetic interactions of HSP90 through functional inhibition by geldanamycin. These findings revealed a vast number of genes that were dependent on HSP90 for normal cellular function, leading to the identification of the varied and essential physiological roles of HSP90 in the cell, and how its interactions influence global patterns of gene expression (Zhao, Davey et al. 2005). In the same way, genetic interactions of the TOR1C complex (the TOR complex 
targeted by rapamycin) were identified by screening yeast deletion mutants for rapamycin sensitivity. This lead to the genome-wide characterization of not only known functions of TOR, but also the construction of additional cellular pathways and processes associated with TOR signalling (Chan, Carvalho et al. 2000).

\subsection{Natural products for drug discovery}

The advent of drug screening technologies and combinatorial synthetic chemistry allowed for the generation of large chemical libraries (Kumar, Kiuchi et al. 2005), from which biologically active compounds could be identified for development as potential drugs. Historically, NP libraries were more difficult to compile due to the need for compound isolation and purification. However, advancement in isolation and structure elucidation techniques (Newman and Cragg 2007) in the latter part of the last century paved the way for competition with synthetic compound libraries in rapidly and robustly identifying biologically active NPs for drug discovery. Most importantly, NPs, unlike synthetic compounds, offered greater structural and chemical diversity, characteristics seemingly intrinsic to biologically active compounds, making them highly desirable in drug discovery research. This, coupled with efficient and robust techniques to identify diverse biologically active NPs saw a marked increase in NP drug discovery, and this is evident in the number of NPs and NP-derived compounds represented as sources of new drugs over the past 25 years (Newman and Cragg 2007). 
To date, more than half of the current pharmacopeia is dominated by NPs or combinatorial compounds derived from natural sources (Figure 1.1). These are unequivocally wide-ranging in their application: for the treatment of bacterial, viral and fungal infections, as immunosuppressants (rapamycin, first isolated from soil bacterium Streptomyces hygroscopicus (Vezina, Kudelski et al. 1975)), as antiinflammatory agents (salicylic acid, from the bark of the willow tree (Raskin 1992)), as well as in treatment of atherosclerosis (lovastatin, a hypolipidemic agent, is produced by several mold species (Moore, Bigam et al. 1985)), and in cancer therapeutics (paclitaxel, a well-established anti-cancer agent, was originally isolated from the bark of the pacific yew tree (Wani, Taylor et al. 1971; Hood, West et al. 2002)).

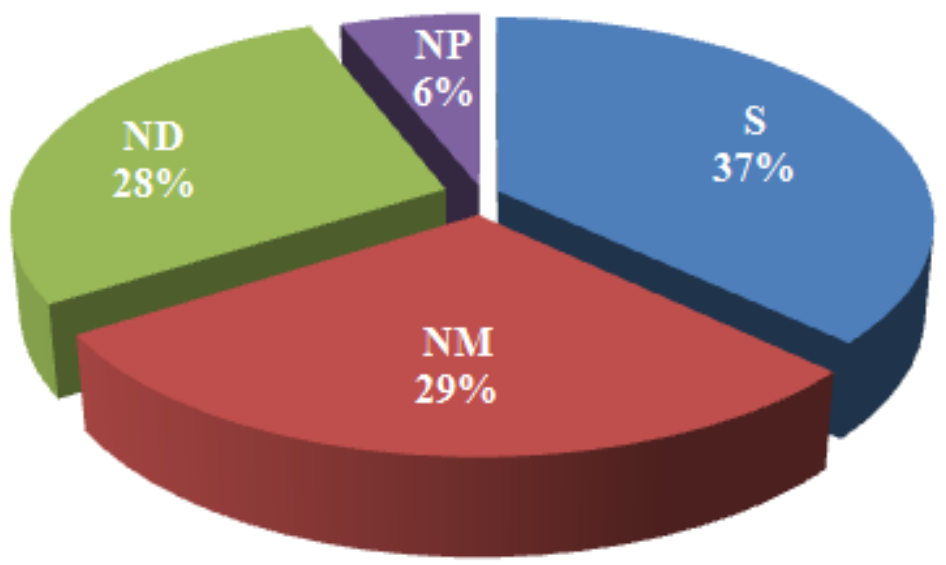

Figure 1.1. All small molecule new entities launched between 1981-2006. (S) indicates purely synthetic compounds, (NM) are synthetic compounds whose pharmacophore were of natural product origin, (ND) are compounds derived from natural products with semi-synthetic modifications, and (NP) indicates pure natural product molecules (Newman and Cragg 2007). 
In comparison to synthetic compounds, NPs possess many pharmacologically attractive properties. Overall, NPs have a greater number of chiral centers and macrocycles, greater structural rigidity, and diverse ring systems (Figure 1.2). Skeletal diversity provides the basis for higher target selectivity and uptake efficiency, and stronger binding properties (Baker, Prior et al. 2002; Feher and Schmidt 2003; Clardy and Walsh 2004; Koehn and Carter 2005). Most importantly, biologically active NPs have evolved alongside their targets, as these molecules are often produced by the host organism to serve some sort of biological function. All these properties of NPs make them highly valuable in drug discovery.
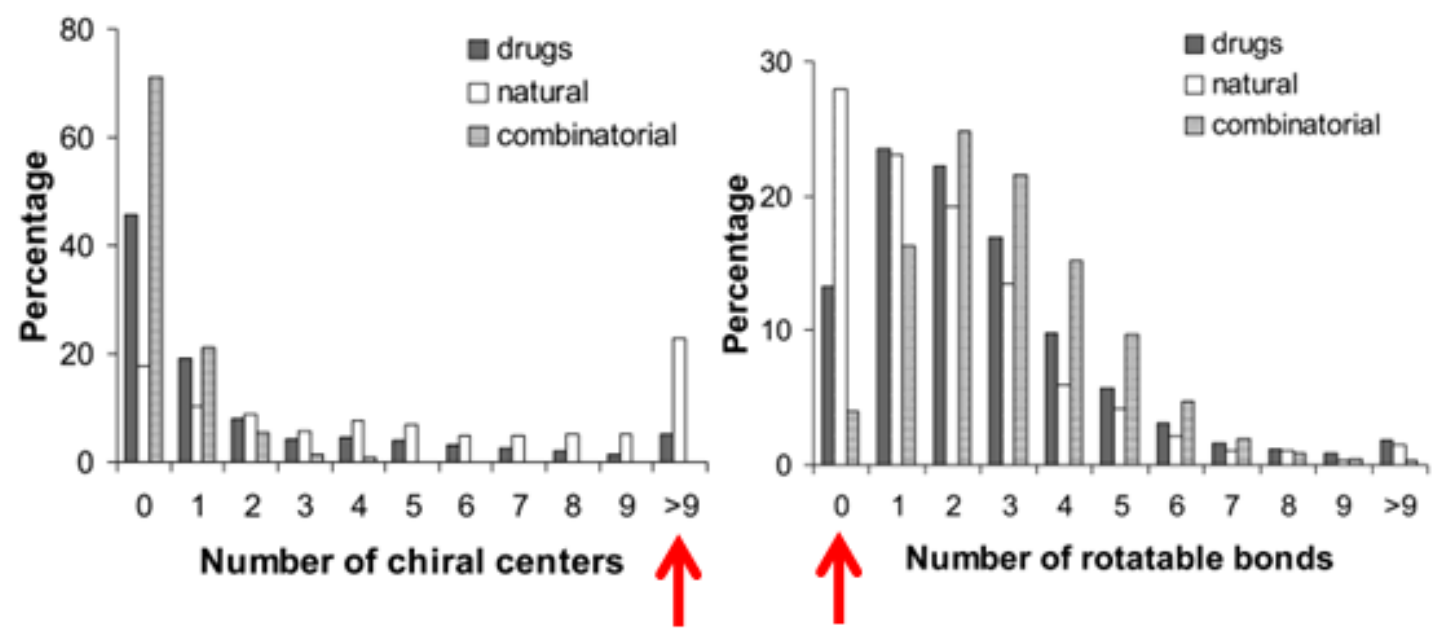

Figure 1.2. Structural diversity and biological specificity of NPs. Overall, NPs have greater number of chiral centers and low freely rotatable bonds, making NPs structurally diverse and possessing higher specificity for target-binding (Blunt, Copp et al. 2008).

The remarkable diversity encompassed by NPs is indisputable in terms of their relevance to drug discovery research, and a number of approaches have been developed to capture their intrinsic value (Gullo, McAlpine et al. 2006). Most importantly, these attributes of NPs have provided modern medicine with a plethora 
of novel drugs in a variety of disease settings, particularly in therapeutic areas with a dearth in leading drugs (Butler 2004; Simmons, Andrianasolo et al. 2005).

\subsection{Yeast in drug discovery}

The yeast Saccharomyces cerevisiae possesses many attributes that distinguish it as an ideal model eukaryotic system for diverse biological studies: it is small and inexpensive to grow, its entire genome has been sequenced, and its $~ 6300$ open reading frames (ORFs) have been made readily usable for genome-wide analyses (Hudson, Dawson et al. 1997; Winzeler, Shoemaker et al. 1999). Yeast as a screening system suffers a drawback for drug discovery due to the extensive drug-efflux pump network (referred to as pleiotropic drug resistance (PDR) pumps) they possess. The presence of these inducible efflux pumps can be challenging, as they lower the effectiveness of a large proportion of drugs (Balzi and Goffeau 1995). For drug candidates that are substrates for this drug-efflux system, the use of sensitized yeast strains wherein drug-efflux is genetically compromised circumvents this problem. Under these circumstances, effective drug concentrations become comparable to those observed in cultured mammalian cell lines (Woo, Kato et al. 1999; Miyamoto, Machida et al. 2002).

Notably, there is high cross-species conservation of genetic components between yeast and humans; $30 \%$ of known genes implicated in human diseases have yeast 
orthologues (Foury 1997; Luesch, Wu et al. 2005), and fundamental cellular processes and metabolic pathways in $S$. cerevisiae are very similar to that of humans (Auerbach, Arnoldo et al. 2005). Thus this cross-species conservation enables the utilization of yeast to discover and characterize the biological activity of compounds that may be of therapeutic relevance in humans. Additionally, the close genetic relations $S$. cerevisiae has to pathogenic fungi such as Candida albicans makes this microorganism an ideal ready-made model system for anti-fungal drug discovery and mode-of-action studies (Hughes 2002).

Many studies have employed $S$. cerevisiae to discover novel small molecule inhibitors (or less frequently, activators) and to characterize their mode-of-action within the microorganism itself. In this way, cincreasin, a small molecule inhibitor of the spindle-checkpoint was identified, and through the use of genome-wide tools available for $S$. cerevisiae, the target of cincreasin, MPS1, a protein kinase required for spindle-checkpoint function was characterized (Dorer, Zhong et al. 2005). Likewise, a class of sirtuin inhibitors were discovered through phenotypic-based chemical-genetic screening in $S$. cerevisiae. Sirtuins are a family of conserved enzymes involved in transcriptional silencing of telomeric loci (Grozinger 2001). These novel compounds were found to selectively inhibit yeast Sir2p activity in vivo and yeast Sir2p and human SIRT2 activity in vitro (Grozinger 2001). These studies demonstrate the utility and robustness of $S$. cerevisiae for drug discovery and drug 
mode-of-action studies. In addition, the findings identified potential targets for therapeutic intervention, molecules that inhibit these targets, and further refinement in understanding these target functions within the cell - this is especially in the case of the sirtuins, wherein all seven human sirtuins remain largely uncharacterized.

The foremost advantage of using $S$. cerevisiae for drug discovery is the availability of tools superlative for drug mode-of-action studies not available in aggregate in any other eukaryote species. The $S$. cerevisiae genome is easily manipulated, and most importantly, genetically tractable. The ease of which yeast undergo homologous recombination, coupled with advanced molecular biology such as robotic nucleotide synthesis has allowed the development of numerous powerful genomic tools. Various yeast libraries have been made available, including a genome-wide set of single deletion knock-outs each identifiable with unique DNA barcodes (Winzeler, Shoemaker et al. 1999), a yeast library of ORFs fused to TAP-tags (tandem affinity purification tags) (Rigaut, Shevchenko et al. 1999; Puig, Caspary et al. 2001) or GFP (green fluorescent protein) (Huh, Falvo et al. 2003), allowing systematic genomewide analyses of protein-protein interactions (Ross-Macdonald, Coelho et al. 1999) and protein localization (Niedenthal, Riles et al. 1996; Huh, Falvo et al. 2003) respectively. Furthermore, the characterization of homozygous and heterozygous gene disruption phenotypes (Tong, Evangelista et al. 2001; Giaever, Chu et al. 2002) 
has been made possible with the yeast deletion mutant libraries (Winzeler, Shoemaker et al. 1999). Many studies have since used these approaches to build a comprehensive understanding of protein regulation, expression, function, and interaction.

In contrast to mammalian-cell drug-target identification which relies heavily upon affinity-based methods to identify potential targets, the long and rich history of yeast molecular genetics methodologies affords the application of many additional approaches to identify drug targets. One of the most successful approaches to identifying drug targets in yeast has been the generation and characterization of drug-resistant mutants. Many studies have employed this approach for drug-target identification; Aureobasidin A is an anti-fungal agent with activity against both Candida sp. and S. cerevisiae (Heidler and Radding 1995). A resistant mutant strain was generated in $S$. cerevisiae to study the mechanism of aureobasidin A. The resistance was linked to a dominant mutation in a single gene, $A U R 1$, a gene encoding phosphatidylinositol:ceramide phosphoinositol transferase (IPC synthase), an enzyme required in sphyngolipid biosynthesis and is essential for the viability of S. cerevisiae (Heidler and Radding 1995).

Often, dominant mutations occur in the target of the compound of interest, and as it was in this case, aureobasidin A potently inhibited IPC synthase activity (Nagiec, 
Nagiec et al. 1997). Moreover, phosphoinositol-containing sphingolipids are unique to fungi, making the sphingolipid biosynthesis pathway an attractive target for antifungal agents (Nagiec, Nagiec et al. 1997; Yuichi S. 2004). Similarly, Thomas et. al. isolated 173 yeast mutants conferring resistance to anti-mitotic agent benomyl. Subsequent mutant characterization revealed all of the resistance conferring mutations had occurred in the same gene, TUB2, which encodes the $\beta$-tubulin subunit of yeast microtubules. Additionally, nine of the mutants conferred temperature-dependent growth, wherein these mutants showed characteristic celldivision-cycle defects at their growth-restrictive temperatures, indicating the importance of tubulin function in cell division. Mapping of TUB2 further described it as the first cell-division-cycle gene that encodes for a cytoskeletal protein with central roles in the cell cycle (Thomas, Neff et al. 1985).

The use of small molecule inhibitors in combination with the readily available genome-wide tools such as the yeast gene deletion sets has also greatly facilitated genetic analyses of a myriad of cellular processes. The discovery of the mitotic spindle checkpoint (Stearns, Hoyt et al. 1990; Chan, Carvalho et al. 2000) and identification of genes required for the correct folding of $\beta$-tubulin (Stearns, Hoyt et. al. 1990), for example, were found through studying the relative sensitivity of yeast mutants to benomyl. In this case, benomyl hyper-sensitive rather than resistant mutants were identified. These studies, in combination with the generation 
of the yeast deletion libraries have paved the way for the recently devised method of chemo-genomic profiling for drug mode-of-action, which will be further discussed in chapter two. 


\section{TETRAMIC ACID 289 AND EQUISETIN}

\subsection{Introduction: Natural products from terrestrial microbes}

Microbial natural products have played a pivotal role in drug discovery during the last century (Larsen, Smedsgaard et al. 2005). Since the discovery of penicillin G from Penicillium sp., interest in fungi, actinomycetes, and other microorganisms soared, and these became a hunting ground for novel drug leads (Larsen, Smedsgaard et al. 2005). Fungi in particular, have provided a plethora of biologically active compounds, many of which were subsequently actively developed and applied as pharmaceuticals and for industrial use (Sims, Fillmore et al. 2005). Compounds are deemed biologically active if they cause a specific response in a biological system. Specific responses detail physical interactions between the compound and a biological structure. More often than not, these responses lead to inhibition of a biological system by the compound; however stimulatory compounds have also been applied. Novel compounds displaying interesting biological activity thus become playgrounds for drug discovery and development.

In this study, a small, focused natural product library of pure compounds isolated from soil fungi provided by our collaborator Dr Stephen Bloor (Biodiscovery NZ, Auckland), were screened for biological activity in yeast, from which two compounds were chosen for further drug-target identification studies; Tetramic acid 289 (TA-289), a novel compound, and a known, structurally-related compound with 
an uncharacterized mode-of-action, equisetin (Figure 2.1). TA-289 and equisetin belong to the tetramic acid class of compounds. Naturally occurring tetramic acids and tetramic acid derivatives constitute a substantial class of compounds; almost all compounds discovered bearing the tetramic acid moiety display some sort of biological activity, the spectrum of which is remarkable in its diversity, ranging from antifungal, to antibacterial, antiviral, to anticancer properties (Royles 1995; Singh, Bisht et al. 2006) - a number of tetramic acid compounds have been screened for biological activity against HIV-1 integrase (Singh, Zink et al. 1998; Hazuda, Blau et al. 1999), gram-positive and -negative bacteria (Vesonder, Tjarks et al. 1979), plants (Wheeler, Stipanovic et al. 1999), fungi (Schobert and Schlenk 2008), and cancer cell lines (Akita 1991; Sugie, al et al. 2002) in vivo and in vitro. This family holds a distinctive structural feature, the 2,4-pyrrolidinedione ring system, a key motif found in many natural products from which biological activity is derived (Singh, Bisht et al. 2006).
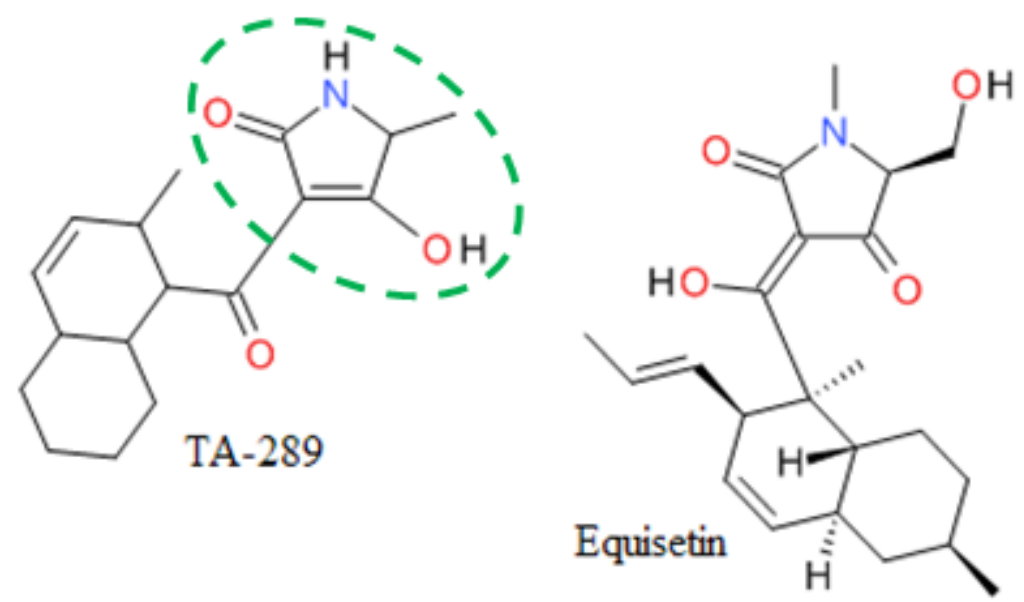

Figure 2.1. Structures of TA-289 and equisetin. Circled in green is the tetramic acid moiety. 
Equisetin, first isolated from Fusarium equiseti (Burmeister, Bennett et al. 1974), has since been shown to be produced by other fungi of the genus Fusarium (Hazuda, Blau et al. 1999; Sims, Fillmore et al. 2005), and has been successfully synthesized (Yuki, Shindo et al. 2001; Burke, Dixon et al. 2005). Equisetin displayed potent inhibitory activity against certain gram positive bacteria, including mycobacteria (Burmeister, Bennett et al. 1974). The compound was also shown to inhibit recombinant HIV-1 integrase in vitro, with reported $\mathrm{IC}_{50}$ values between 7-20 $\mu \mathrm{M}$ (Singh, Zink et al. 1998). Recently, a study on trypanosomal post-translation protein import showed that equisetin displayed inhibitory activity against translocation of post-translational proteins to the endoplasmic reticulum $\left(\mathrm{IC}_{50} 3.3 \mu \mathrm{M}\right)$ in Trypanosoma brucei (Patham, Duffy et al. 2009).

Studies conducted by König et. al. in isolated rat mitochondria found equisetin potently inhibited the uptake of succinate, ATP (adenosine triphosphate), $\mathrm{P}_{\mathrm{i}}$ (inorganic phosphate), and succinate in a dose-dependent manner. ATPase activity and $\mathrm{P}_{\mathrm{i}}$ exchange were also significantly decreased in the presence of equisetin. In this study, equisetin was proposed to specifically inhibit the substrate anion carriers of the inner mitochondrial membrane, however the exact mechanism by which equisetin inhibits these carriers has not been established (König, Kapus et al. 1993). Lundin et. al. showed an increase in inorganic mitochondrial pyrophosphatase (PPase) activity by equisetin in $S$. cerevisiae (Lundin, Deopujari et al. 1992). To date, 
the study conducted by Lundin et. al. is the only one to provide evidence of equisetin biological activity in $S$. cerevisiae. Another study examined the effects of equisetin on energy-linked reactions of chromatophores of the gram negative proteobacterium Rhodospirillum rubrum, and concluded a nonspecific action of equisetin on membranes and hydrophobic domains of proteins (Nyren and Strid 1989). Currently, the mechanism-of-action of equisetin remains uncharacterized.

Numerous compounds containing tetramic acid moieties have been isolated from various fungi, however there appears to be a distinct sub-class of equisetin-like compounds (Figure 2.2), all of which displayed potent antimicrobial activity against a number of gram positive bacteria (Staphylococcus sp., Pseudomonas sp., Enterococcus sp., Bacilli sp., and Streptococcus sp.), certain pathogenic fungi including Candida albicans (coniosetin), Cladosporium resinae and Trytophyton mentagrophytes (paecilosetin), and HeLa (CJ-17-572, CJ-21-058) and P388 (paecilosetin) tumorgenic cell lines (Schobert and Schlenk 2008). Trichosetin was found to be phytotoxic, inhibiting the growth of roots and shoots in various seedlings, as was equisetin (Wheeler, Stipanovic et al. 1999). Moreover, a dosedependent increase in electrolyte leakage and lipid peroxidation caused by trichosetin, resulting in damaged mitochondria has been observed (Marfori, Kajiyama et al. 2003). As with equisetin, CJ-17-572 and CJ-21-058 inhibit SecA activity in vitro. SecA is part of the eubacterial translocase pathway, involved in 
translocating pre-proteins and mature proteins across membranes within the cell (Bieker 1990; Sugie, al et al. 2002). The reported activities of these structurally related compounds may indicate possible similar mechanisms-of-action between TA-289 and equisetin, where often, structurally similar compounds have been shown to be comparable in their mechanisms (Ghannoum and Rice 1999). 
A.<smiles>C[C@@H]1NC(O)=C(C(=O)[C@]2(C)[C@@H](C)C=C[C@H]3CCCC[C@@H]32)C1=O</smiles>

B.<smiles>C/C=C/[C@H]1C=C[C@H]2C[C@@H](C)CC[C@H]2[C@]1(C)/C(O)=C1\C(=O)[C@H](CO)N(C)C1=O</smiles><smiles>C/C=C/[C@H]1C=C[C@H]2C[C@@H](C)CC[C@H]2[C@]1(C)/C(O)=C1/C(=O)N[C@H](CO)C1=O</smiles>

2.<smiles>C/C=C/C=C/[C@H]1C=C[C@H]2C[C@@H](C)CC[C@H]2[C@]1(C)/C(O)=C1/C(=O)N[C@H](C(C)O)C1=O</smiles>

5.<smiles>CC1=C[C@H]2C[C@@H](C)CC[C@H]2[C@@](C)(C(O)=C2C(=O)[C@H](C(C)O)N(C)C2=O)C1</smiles>

3.<smiles>C/C=C/C=C/[C@H]1C(C)=C[C@H]2C[C@H](C)CC[C@H]2[C@@]1(C)/C(O)=C1\C(=O)[C@H](CO)N(C)C1=O</smiles>

6.<smiles>C/C=C/C=C/[C@H]1C(C)=C[C@H]2CCCC[C@]2(C(=O)C2=C(O)[C@@H](CO)N(C)C2=O)C1(C)C</smiles>

7.<smiles>C/C=C/C=C/C1C(C)=C[C@H]2C[C@@H](C)CC[C@@H]2C1(C)/C(O)=C1\C(=O)N[C@H](C(C)O)C1=O</smiles>

8.<smiles>C/C=C/C1C(C)=C[C@H]2C[C@@H](C)CC[C@H]2[C@]1(C)/C(O)=C1\C(=O)[C@H](C(C)O)N(C)C1=O</smiles>

Figure 2.2. Chemical structures of structurally-related tetramic acid compounds. A) TA-289, B) Equisetin (Burmeister, Bennett et al. 1974; Vesonder, Tjarks et al. 1979), 1) Trichosetin (Marfori 2002), 2) Altersetin (Hellwig, Grothe et al. 2003), 3) Phomasetin (Cabelli 1991), 4) CJ-21-058 (Sugie, al et al. 2002), 5) CJ-17-572 (Akita 1991), 6) LL-49F233 $\alpha$ (Singh, Zaccardi et al. 1998), 7) Coniosetin (Schobert and Schlenk 2008), 8) Paecilosetin (Schobert and Schlenk 2008). 


\subsection{The yeast genome deletion set}

Following the complete genome sequencing of $S$. cerevisiae was the generation of a comprehensive set of yeast deletion strains, that is, the creation of a deletion strain for each open reading frame (ORF) in the yeast genome. As part of the deletion process, every ORF was replaced with a kanamycin-resistance cassette. In addition, each deletion was uniquely identified by the addition of a pair of $20 \mathrm{bp}$ nucleotide sequence "barcodes". Moreover, universal primer sequences flanking the unique "barcodes" allowed for mass PCR amplification and quantification of abundance of an individual mutant in a mixed population of mutant strains i.e. microarray-based phenotypic analyses (Boone, Bussey et al. 2007). From this, four yeast deletion libraries were produced: the homozygous deletion set wherein each strain is either mating type MATa, MAT $\alpha$, or as a diploid, has a non-essential gene replaced with the kanamycin-resistance cassette; the heterozygous deletion set comprising strains where one copy of each essential gene has been replaced with the kanamycinresistance cassette.

To date, each of these collections, highly valued in the yeast community as key resource tools used for large scale and systematic genetics analyses, have been employed for drug target identification and/or mode-of-action studies. In general, the expression levels of a protein can be decreased by approximately half through the deletion of a copy of a gene in a diploid yeast strain, leading to hypersensitivity to an inhibitory compound (Giaever, Shoemaker et al. 1999); conversely, the 
opposite can be achieved, where gene target overexpression confers drug resistance (Rine, Hansen et al. 1983; Launhardt, Hinnen et al. 1998). This approach exploits the concept that the gene target of a compound of interest can be determined through analysis of mutants that are hyper-sensitive to the compound compared to that of wild-type cells (Giaever, Shoemaker et al. 1999). While the use of the heterozygous deletion library takes into account nearly every gene in drug sensitivity, there is variability in the success of unambiguously determining drug targets, and that the most drug-sensitive strain does not always reveal the direct drug target (Sturgeon, Kemmer et al. 2006).

Studies involving the use of the homozygous deletion library in determining the mode-of-action of a compound of interest are based on the fact that the deletion of a gene results in a complete lack of expression of associated nonessential gene products. In principle, changes in drug sensitivity are a result of deletions in nonessential genes whose products functionally interact with the drug target (Sturgeon, Kemmer et al. 2006). In this way, Chan et al. screened 2, 226 nonessential deletion mutants with target-of-rapamycin (TOR) inhibitor rapamycin, and identified 106 mutants that displayed an increased sensitivity (Chan, Carvalho et al. 2000). Within these identified mutants were those previously shown to have rapamycin sensitivity; however the additional mutants found in this study 
broadened the knowledge of the cellular functions and components of the TOR pathway.

While homozygous genetic profiling rarely identifies drug targets directly - essential genes are not represented in this library, and many small molecule inhibitors target essential genes in yeast, this approach provides an unprecedented and comprehensive view of cellular pathways and processes that are functionally affected by the small molecule (Sturgeon, Kemmer et al. 2006). Parsons et al. compared the chemical genomic screen profiles of 12 different compounds, to a collection of synthetic-lethal interaction profiles in order to decipher more precisely potential target pathways. For example, the profile of camptothecin, one of the 12 drugs screened, was similar to the profiles obtained for DNA synthesis genes, allowing for identification of the compound's probable mode-of-action even when the direct target was not represented in the gene cluster (Parsons, Brost et al. 2004).

As more comprehensive databases and knowledge on genetic interaction networks grow and more cellular pathways are uncovered, the utility of both the heterozygous and the homozygous deletion library, screened in concert against a compound of interest will result in very powerful and informative tools for drugtarget identification in yeast (Sturgeon, Kemmer et al. 2006). 


\subsection{Aims and objectives}

The aim of this chapter was to establish basic biological activities of TA-289 and equisetin in yeast, and to ascertain a possible mechanism and/or site-of-action for both compounds. These included:

- Determining MIC of TA-289 and equisetin in liquid and solid media under different $\mathrm{pH}$ and media conditions

- Cell morphology and cell cycle analysis

- Chemical genomic profiling of TA-289 utilizing the heterozygous diploid and the homozygous diploid yeast genome deletion sets 


\subsection{Materials and methods}

\subsubsection{Yeast strains}

All $S$. cerevisiae strains used in this study are as follows:

\begin{tabular}{|c|c|}
\hline Strain & Genotype \\
\hline YCG100 (BY4743) & 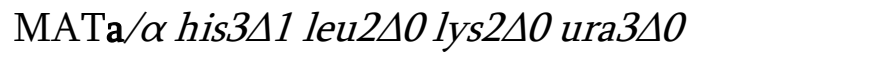 \\
\hline YCG104 (BY4742, wt) & 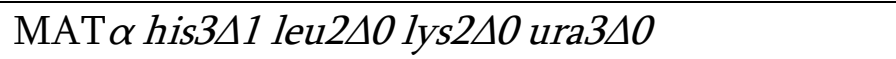 \\
\hline YCG289 (yeGFP) & 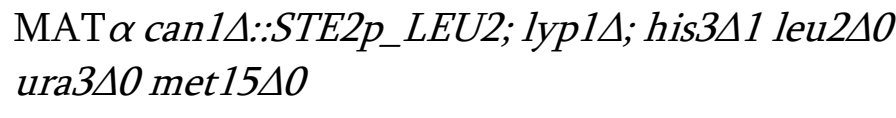 \\
\hline YCG326 $(p d r \Delta)$ & 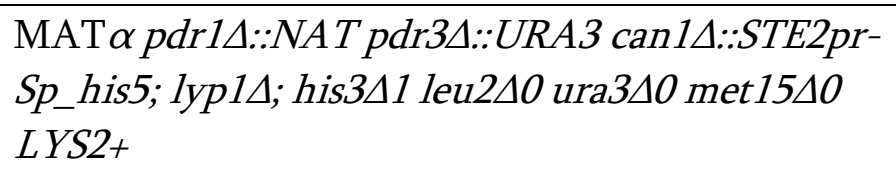 \\
\hline$t u b 2 \Delta$ & $\begin{array}{l}\text { Heterozygous diploid TUB2/tub2 }:: \text { kanR; his3 } 11 \\
\text { leu2 } \Delta 0 \text { ura3 } \Delta 0 \text { met } 15 \Delta O\end{array}$ \\
\hline
\end{tabular}

\subsubsection{Growth media}

All yeast cultures were performed in one of the following media below supplemented with either geneticin ((G418) $200 \mu \mathrm{g} / \mathrm{mL}$ final concentration), nourseothricin ((NAT) $100 \mu \mathrm{g} / \mathrm{mL}$ final concentration), or canavanine ((Can) 50 $\mu \mathrm{g} / \mathrm{mL}$ ) and thialysine (S-aminoethyl-L-cysteine hydrochloride (Thia); $50 \mu \mathrm{g} / \mathrm{mL}$ ) (Sigma-Aldrich, MO, USA), unless otherwise stated. For solid media preparation, 2\% (w/v) agar was added (denoted as “-agar”):

Yeast peptone dextrose (YPD): $1 \%(\mathrm{w} / \mathrm{v})$ yeast extract, $2 \%(\mathrm{w} / \mathrm{v})$ bacto-peptone, $2 \%$ $(\mathrm{w} / \mathrm{v})$ glucose. 
Synthetic complete (SC): $0.17 \%(\mathrm{w} / \mathrm{v})$ bacto-yeast nitrogen base (without amino acids or ammonium sulphate), $0.1 \%(\mathrm{w} / \mathrm{v})$ monosodium glutamate, $0.2 \%(\mathrm{w} / \mathrm{v})$ “amino acid mix" (Amberg 2005), 2\% (w/v) glucose.

Synthetic drop-out (SD) -Ura: as with SC but without uracil in the "amino acid mix".

SD -His/Arg/Lys: as with SC but without histidine, arginine, and lysine in the "amino acid mix".

Pre-sporulation (GNA): $1 \%(\mathrm{w} / \mathrm{v})$ yeast extract, $3.8 \%(\mathrm{w} / \mathrm{v})$ bacto-peptone, $12.5 \%$ $(\mathrm{w} / \mathrm{v})$ glucose.

Enriched sporulation (SPO): $1 \%(\mathrm{w} / \mathrm{v})$ potassium acetate, $0.1 \%(\mathrm{w} / \mathrm{v})$ yeast extract, $0.05 \%(\mathrm{w} / \mathrm{v})$ glucose, $0.01 \%(\mathrm{w} / \mathrm{v})$ "SPO amino acid mix" (Amberg 2005).

\subsubsection{Compounds and chemicals}

Received from Dr. Stephen Bloor (Biodiscovery New Zealand, Industrial Research Limited, Wellington); 88 natural product isolates derived from soil fungi Fusarium $s p$. The 88 compounds, approximated at $400 \mu \mathrm{g}$ each in mass, were provided in a 96well tissue culture plate, into which 100\% dimethyl sulfoxide (DMSO) was added to make $\approx 10 \mathrm{mM}$ working stocks of each compound. The compounds were stored at $20{ }^{\circ} \mathrm{C}$ until further use. Additional fractions of tetramic acid 289 (TA-289) and equisetin were provided (9.6 $\mathrm{mg}$ and $14.4 \mathrm{mg}$, respectively), and $10 \mathrm{mM}$ working stocks of both compounds were prepared by dissolving in DMSO and then stored at $-20^{\circ} \mathrm{C}$ until further use.

Mollisoside, kindly provided by Dr Peter Northcote (School of Chemical and Physical Sciences, Victoria University of Wellington), was prepared at a $1 \mathrm{mM}$ working stock in DMSO and stored at $-20^{\circ} \mathrm{C}$. 
Purchased from Sigma-Aldrich Life Science and Biochemicals: cycloheximide, $10 \mathrm{mg} / \mathrm{mL}$ working stock stored at $-20^{\circ} \mathrm{C}$, benomyl, $10 \mathrm{mg} / \mathrm{mL}$ working stock stored at $-20^{\circ} \mathrm{C}$, and $\mathrm{DMSO}$, stored at room temperature.

\subsubsection{Growth inhibition in liquid media}

Two yeast strains, wild-type BY4742 (wt) and YCG326, a mutant strain deficient in the two main transcription factors of the yeast drug efflux pump network, $P D R 1$ and PDR3 (pdrA) were grown in $2 \mathrm{~mL} \mathrm{SC}$ broth overnight at $30{ }^{\circ} \mathrm{C}$ on a rotating drum. The cultures were subsequently counted on a haemocytometer, further diluted to $5 \times 10^{5}$ cells $/ \mathrm{mL}$ in fresh SC, after which $100 \mu \mathrm{L}$ aliquots were dispensed into a 96well tissue culture plate to give a final concentration of $5 \times 10^{4}$ cells $/ \mathrm{mL}$. A $1 \mu \mathrm{L}$ aliquot of each of the 88 compounds (10 mM working stock concentration) was added to the culture plate to give a final concentration of $100 \mu \mathrm{M}$ of each compound. The plate was mixed by vortexing, then incubated at $30{ }^{\circ} \mathrm{C}$ for $18 \mathrm{~h}$, after which cell growth was quantified by measuring optical density (OD) at $590 \mathrm{~nm}$ using a Wallac EnVision 2102 Multilabel Plate Reader (Perkin Elmer ${ }^{\mathrm{TM}}$, Waltham, MA, USA.).

\subsubsection{Liquid dose-response growth assays}

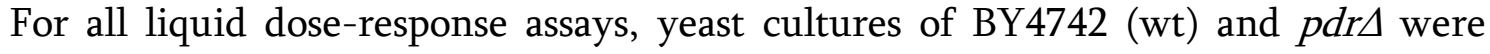
grown overnight in $2.5 \mathrm{~mL}$ SC media at $30{ }^{\circ} \mathrm{C}$ on a rotating drum. The cell concentrations of each culture were determined by haemocytometer and diluted to 
$5 \times 10^{5}$ cells $/ \mathrm{mL}$ in one of four different media solutions: 1 ) SC (normal $\mathrm{pH} \sim 4.0$ ), 2) pH 8.0 SC, buffered with 25mM HEPES (SC-H), 3) YPD (normal pH 7.0), and 4) $\mathrm{pH}$ 4.0 YPD, adjusted with 18.6 M hydrochloric acid (YPD-HCl). Then, $100 \mu \mathrm{L}$ aliquots of each media solution were dispensed into 96-well tissue culture plates to give a final concentration of $5 \times 10^{4}$ cells $/ \mathrm{mL}$.

An eight-point serial dilution of TA-289 and equisetin were prepared from the $10 \mathrm{mM}$ working stocks, from which a $1 \mu \mathrm{L}$ aliquot of each dilution point was added in triplicate to the wells containing $100 \mu \mathrm{L}$ SC or SC-H or YPD or YPD-HCl media with yeast, to achieve a final concentration range of $100 \mu \mathrm{M}, 50 \mu \mathrm{M}, 30 \mu \mathrm{M}, 25 \mu \mathrm{M}$, $10 \mu \mathrm{M}, 5 \mu \mathrm{M}, 3 \mu \mathrm{M}$, and $1 \mu \mathrm{M}$ TA-289 and equisetin. DMSO (1\% final concentration) served as a solvent control.

For liquid dose-responses using the tub2 $2: K a n R$ heterozygous diploid strain $(t u b 2 \triangle)$, a mutant strain deficient in one gene copy of TUB2, the yeast homologue of $\beta$-tubulin, overnight cultures of BY4743 and tub2 $\Delta$ were diluted in fresh SC media and dispensed into 96-well tissue culture plates as above. Eight-point serial dilutions of TA-289 and equisetin were prepared as described in the previous method and $1 \mu \mathrm{L}$ aliquots were added to the wells as per above, with $1 \%$ DMSO serving as a solvent control. Eight-point serial dilutions of benomyl was prepared from a $10 \mathrm{mg} / \mathrm{mL}$ working stock, giving a final concentration range of $100 \mu \mathrm{g} / \mathrm{mL}, 50 \mu \mathrm{g} / \mathrm{mL}$, $30 \mu \mathrm{g} / \mathrm{mL}, 25 \mu \mathrm{g} / \mathrm{mL}, 10 \mu \mathrm{g} / \mathrm{mL}, 5 \mu \mathrm{g} / \mathrm{mL}, 3 \mu \mathrm{g} / \mathrm{mL}$, and $1 \mu \mathrm{g} / \mathrm{mL}$. As with TA-289 and 
equisetin, $1 \mu \mathrm{L}$ aliquots of benomyl diluents were added to wells containing BY4743 and tub2 $\Delta$. Benomyl served as a positive control.

Plates were mixed by vortexing and incubated at $30{ }^{\circ} \mathrm{C}$ for $18 \mathrm{~h}$. Cell growth was quantified by measuring optical density (OD) at $590 \mathrm{~nm}$.

\subsubsection{Solid dose-response growth assays}

Eight-point serial dilutions of TA-289 and equisetin were prepared from $10 \mathrm{mM}$ working stocks dissolved in DMSO as in the liquid dose response assays. A $10 \mu \mathrm{L}$ volume of drug diluent was added to 24-well tissue culture plates containing $1 \mathrm{~mL}$ of SC-agar, giving a final concentration range of $100 \mu \mathrm{M}-1 \mu \mathrm{M}$. DMSO (1\% final concentration) served as a carrier control. Yeast cultures of BY4742 and pdrA were grown in $2 \mathrm{~mL}$ SC media overnight at $30^{\circ} \mathrm{C}$, after which the cultures were counted using a haemocytometer and then further diluted to $5 \times 10^{5}$ cells $/ \mathrm{mL}$ in $\mathrm{ddH}_{2} \mathrm{O}$. To each agar well, $2 \mu \mathrm{L}$ aliquots of the cell suspension were added, giving a total concentration of 200 cells per well. The plates were incubated at $30{ }^{\circ} \mathrm{C}$ for 2 days, after which they were photographed using a Canon Powershot S3-IS camera and ZoomBrowser EX remote shooting software (Canon Inc., NY, USA). Cell growth was determined by visual inspection. 


\subsubsection{Colony forming unit assay}

Briefly, BY4742 and $p d r \Delta$ yeast cultures were grown overnight in $2 \mathrm{~mL}$ SC media at $30{ }^{\circ} \mathrm{C}$ on a rotating drum, counted using a haemocytometer and further diluted to $5 \times 10^{5}$ cells/mL in fresh SC, after which $100 \mu \mathrm{L}$ aliquots were dispensed in triplicate into 96-well tissue culture plates. A $1 \mu \mathrm{L}$ aliquot of either $10 \mathrm{mM}$ TA-289, $10 \mathrm{mM}$ equisetin, $0.1 \mathrm{mM}$ cycloheximide, and $1 \mathrm{mM}$ mollisoside, were added to the culture plates, giving final concentrations of $100 \mu \mathrm{M}, 100 \mu \mathrm{M}, 1 \mu \mathrm{M}$, and $10 \mu \mathrm{M}$, respectively. In addition, 1\% DMSO served as a second positive carrier control.

The plates were mixed by vortexing, and then incubated at $30{ }^{\circ} \mathrm{C}$ for $18 \mathrm{~h}$. Following this, $100 \mu \mathrm{L}$ aliquots from each drug-treatment were transferred to sterile $1.5 \mathrm{~mL}$ microcentrifuge tubes and cells were collected by centrifugation and the cell pellets thrice-washed with $\mathrm{ddH}_{2} \mathrm{O}$. The washed cells were then counted using a haemocytometer and diluted to $5 \times 10^{3}$ cells $/ \mathrm{mL}$, and $10 \mu \mathrm{L}$ from each sample tube was plated onto petri plates containing YPD-agar, to give 50 cells per plate. The plates were incubated at $30{ }^{\circ} \mathrm{C}$ for 2 days, after which they were photographed, and cell recovery assessed by the presence of colonies on each plate.

\subsubsection{Cell morphology}

Cells from the end-point liquid dose-response assays were assessed for morphological changes using an inverted phase contrast microscope at $60 \mathrm{X}$ magnification (Olympus NZ PTY Ltd, Auckland, NZ) and then photographed (Nikon TA Macalister Ltd, Auckland, NZ). Both BY4742 and pdrA cells treated at 
$100 \mu \mathrm{M}$ and $30 \mu \mathrm{M}$ of TA-289, as well as the DMSO control were photographed. Control cells were diluted 100 fold prior to photographing for ease of manual cell counting. Cell diameter of a random sample of 50 cells from each treatment were measured on ImageJ (Image Processing and Analysis in Java, National Institutes of Health). A two-tailed t-test assuming equal variances was performed, and differences in cell size of treated vs. untreated were calculated.

\subsubsection{Cell cycle analysis by flow cytometry}

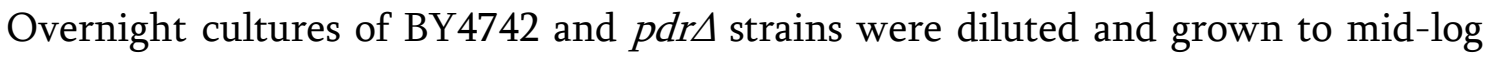
phase (cell concentration as determined on a haemocytometer; approximately $2 \times 10^{7}$ cells $/ \mathrm{mL}$ ) in $50 \mathrm{~mL}$ SC media, after which $0.5 \mathrm{~mL}$ aliquots were dispensed into sterile $15 \mathrm{~mL}$ falcon tubes. A $5 \mu \mathrm{L}$ aliquot of TA-289 and equisetin taken from working stocks of $10 \mathrm{mM}$ was added per tube to give a final concentration of $100 \mu \mathrm{M}$. DMSO (1\% final concentration) served as a carrier control. The treated and untreated cultures were mixed by vortexing and incubated for $6 \mathrm{~h}$ at $30^{\circ} \mathrm{C}$. Cells were collected by centrifugation for $5 \mathrm{~min}$ at $900 \mathrm{x} g$ at room temperature, then

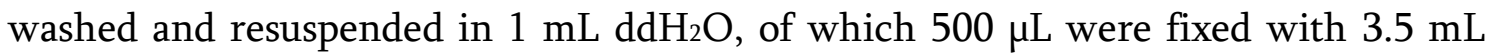
95\% ethanol. Fresh tubes containing $2 \mathrm{~mL}$ SC media were inoculated with the remaining $500 \mu \mathrm{L}$ of washed cells, and incubated at $30{ }^{\circ} \mathrm{C}$ on a rotating drum. A $500 \mu \mathrm{L}$ aliquot of cells were harvested and fixed in ethanol every 30 mins for $1.5 \mathrm{~h}$. After the last harvest, all cell cultures were incubated overnight at $4{ }^{\circ} \mathrm{C}$. 
Cells were collected by centrifugation and washed twice with $\mathrm{ddH}_{2} \mathrm{O}$. After a second centrifugation at $10000 \mathrm{x}$ g, cells were resuspended in $1 \mathrm{~mL}$ fresh $1 \mathrm{X}$ RNase solution

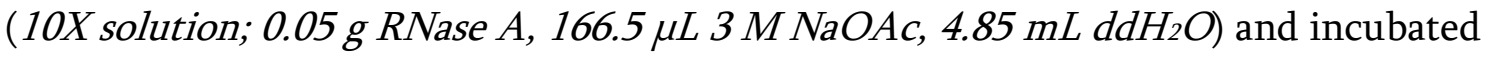
for $3 \mathrm{~h}$ at $37^{\circ} \mathrm{C}$. Cells were recovered by centrifugation, supernatant discarded, and pellets resuspended in $500 \mu \mathrm{L}$ fresh pepsin solution $(1 \mathrm{~mL}$ solution; $5 \mathrm{mg}$ pepsin, $55 \mu L 1 N$ HCl, $945 \mu L d d H 2 O$ ) an incubated for 5 mins at room temperature. Cells were collected by centrifugation, resuspended in $500 \mu \mathrm{L} 50 \mathrm{mM}$ Tris $(\mathrm{pH}$ 7.5), and stored overnight at $4{ }^{\circ} \mathrm{C}$.

Cells were stained with SYTOX Green nucleic acid stain (for each sample, $400 \mu \mathrm{L}$ 50 mM Tris (pH 7.5) containing $2 \mu \mathrm{M}$ SYTOX Green) (Haase 2001; Amberg 2005). A $400 \mu \mathrm{L}$ aliquot of cells were stained with $400 \mu \mathrm{L}$ SYTOX Green, and cell samples were sorted through the FACScan flow cytometer (BD Biosciences). Cell cycle stages were gated, analysed, and histogram plots were generated for each treatment condition with CellQuest Pro software (BD Biosciences).

\subsubsection{Determining the presence of cell populations by flow cytometry}

Cell concentrations of overnight cultures of the yeGFP strain were determined by measuring OD at $600 \mathrm{~nm}$ using a spectrophotometer (Genova Life Science, Bibby Scientific Ltd, Staffordshire, UK). These were then diluted to $5 \times 10^{5}$ cells/mL in fresh SC media. A $100 \mu \mathrm{L}$ aliquot was taken from both BY4742 and pdrA cells treated at the highest concentration of TA-289 and equisetin after $18 \mathrm{~h}$ treatment as described in the liquid dose-response assays. Into this, a $100 \mu \mathrm{L}$ aliquot of the diluted 
yeGFP culture was added, and then $400 \mu \mathrm{L} d \mathrm{dH}_{2} \mathrm{O}$. Cells were visualized on the FACScan flow cytometer (BD Biosciences, MD, USA) and contour plots were generated for each sample with CellQuest Pro software (BD Biosciences).

\subsubsection{Haplo-insufficiency and homozygous profiling}

Two chemical genomic profiling techniques utilizing the heterozygous diploid deletion set and the homozygous diploid deletion set were carried out with TA-289. These were performed according to the yeast tag microarray protocol (Rosemary Heathcott, personal communication, ESR Wellington) adapted from (Parsons, Lopez et al. 2006). A repeat microarray was performed using the homozygous diploid deletion set for TA-289.

\subsubsection{Microarray analysis}

The microarray chips were read on an Axon Microarray Scanner, where fluorescently labeled control tags were scanned at $532 \mathrm{~nm}$ and experimental samples (drug-treated) scanned at $635 \mathrm{~nm}$ (Genomics Facility, Biochemistry Department, Otago University).

The intensities of the up (UP) and down (DN) tags were normalized and $\log _{2}$ ratios of drug-treated/DMSO control data were obtained using Standardization and Normalization of Microarray Data (SNOMAD) software (http://pevsnerlab. kennedykrieger.org/snomadinput.html). Gene tags with a low fluorescent control 
signal (less than 500) were removed, the remaining log ratios of tag replicates were averaged, and deviation (D) scores calculated $(D$ score $=$ (average of log ratios - mean of average of log ratios)/standard deviation of average of log ratios). Genetic and physical interactions and gene descriptions of selected gene "hits" (D score $=<-3$, and $>-1)$ were batch-downloaded from the yeast genome database (http://www.yeastgenome.org). Interaction networks were created in Cytoscape open source platform for complex network analysis and visualization, $\mathrm{v} 2.8 .1$ ((Shannon, Markiel et al. 2003), Cytoscape Consortium). Hierarchical cluster analyses were carried out following Hillenmeyer et. al. 2008. Gene tags were grouped according to intracellular localization and compared to the yeast genome by taking random subsets of genes and statistically determining the significance of each localization, assuming a normal distribution of sampling.

\subsubsection{Cell death assays}

Cultures of BY4742, $p d r \Delta$, and TA8 were prepared by inoculating either $2 \mathrm{~mL}$ SC, or $2 \mathrm{~mL}$ SC-gly-EtOH (SC with 2\% glycerol and 2\% ethanol, instead of glucose) and grown overnight at $30{ }^{\circ} \mathrm{C}$ on a rotating drum. Cell concentrations of each culture were determined by measuring OD at $600 \mathrm{~nm}$ and subsequently diluted in either SC or SC-gly-EtOH to give a final cell concentration of $1 \times 10^{6}$ cells $/ \mathrm{mL}$.

For the BY4742 and pdrA cell death assays with TA-289 and equisetin in SC-gly$\mathrm{EtOH}$, a $100 \mu \mathrm{L}$ aliquot of diluted cells in SC-gly-EtOH were dispensed into 96-well tissue culture plates, after which $1 \mu \mathrm{L}$ aliquots of the previously described eight- 
point serial dilutions of either TA-289 or equisetin were added, giving a final concentration range of $100 \mu \mathrm{M}$ to $0.03 \mu \mathrm{M}$. After addition of the compounds, the plates were mixed by vortexing, and a $1.5 \mu \mathrm{L}$ aliquot of the treated cells were removed and spotted onto a Singer ${ }^{\odot}$ plate containing SC. The plates were then incubated at $30^{\circ} \mathrm{C}$. A $1.5 \mu \mathrm{L}$ aliquot of cells were harvested and spotted onto the SC plate every 15 mins for the first 30 mins, and then every 30 mins for $2 \mathrm{~h}$.

For BY4742 and pdrA cell death assays in $\mathrm{H}_{2} \mathrm{O}_{2}$ with SC-gly-EtOH, a $100 \mu \mathrm{L}$ aliquot of diluted cells in SC-gly-EtOH were dispensed into 96-well tissue culture plates, after which $1 \mu \mathrm{L}$ aliquots of the previously described eight-point serial dilution of $\mathrm{H}_{2} \mathrm{O}_{2}$ was added, giving a final concentration range of $9 \mathrm{mM}$ to $3 \mathrm{mM}$. The assay was performed as per cell death assays described above, however cells were harvested every hour for $4 \mathrm{~h}$.

After the final harvest, plates were allowed to dry, and then incubated for two days at $30{ }^{\circ} \mathrm{C}$. Photographs of the plates were taken on both day one and day two of incubation, and growth of colonies were assessed manually. 


\section{$\underline{2.5 \text { Results }}$}

\subsubsection{Initial screening of natural product isolates}

Biological activities of the soil microbial metabolites (88 compounds in total) were assessed by liquid growth inhibition assays, where observation of growth inhibition indicated biological activity and therefore cellular targets in yeast. Of the 88 compounds screened, 22 were identified as biologically active in BY4742 (wt) cells (>70\% growth inhibition compared to DMSO carrier controls) (Figure 2.3).

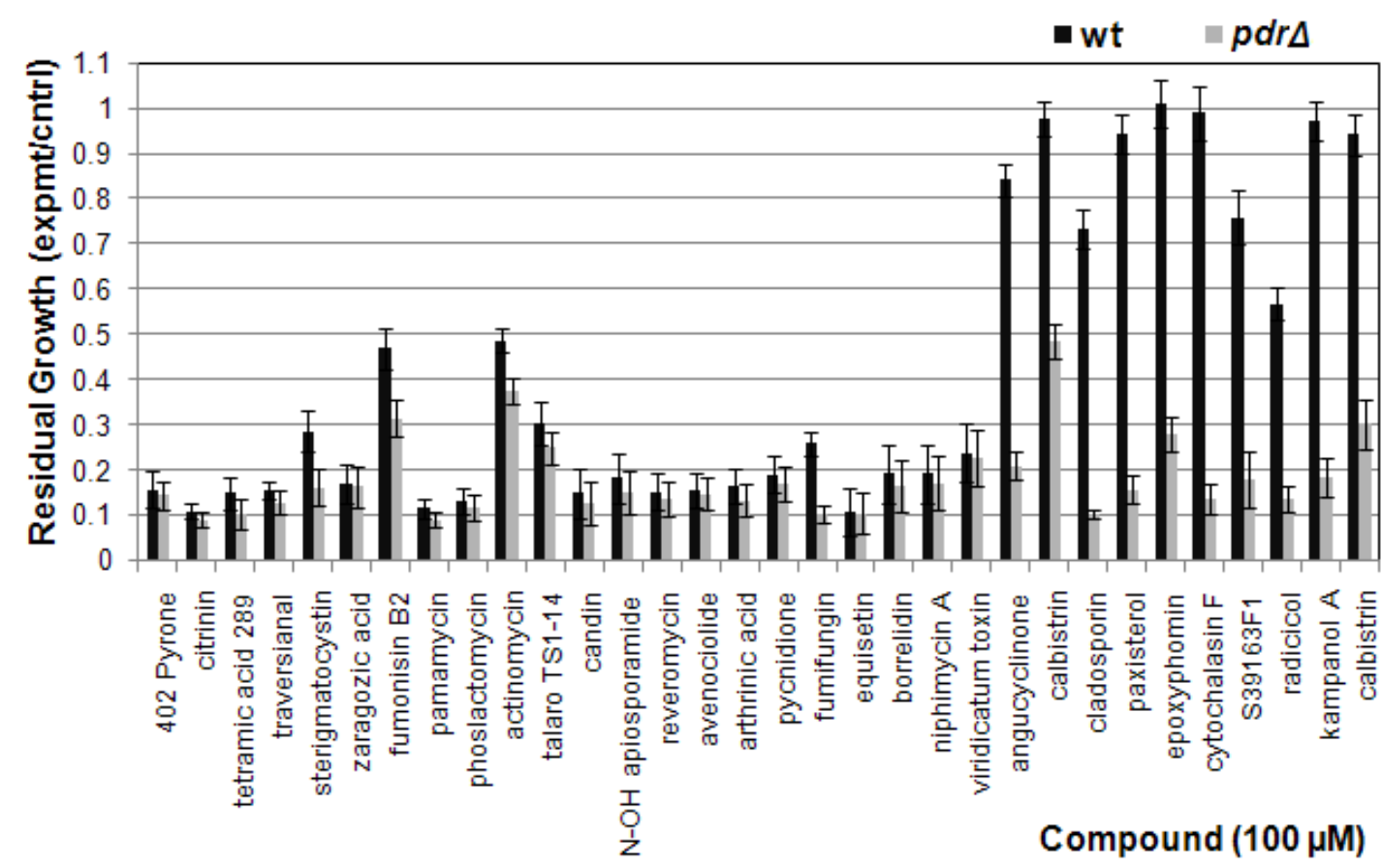

Figure 2.3. Bioactive natural product isolates display differential activity depending on the PDR state of the yeast cell. Liquid growth inhibition assays were performed using two yeast strains, wt and $p d r \Delta$, tested against 88 natural product isolates at $100 \mu \mathrm{M}$. Bioactivity of each compound was determined by calculating residual growth (experiment/control) (y-axis). The graph shows the 32 small molecules deemed as bioactive. Of these, 22 displayed no apparent difference in potency against the two yeast strains tested (402 pyrone - viridicatum toxin). On the contrary, ten compounds (angucyclinone - calbistrin; far right compounds) showed significant difference in potency levels between the two strains. 


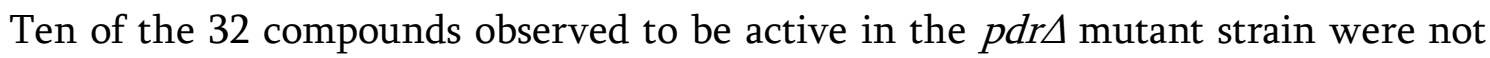
active in the BY4742 (wt) strain (Figure 2.3; angucyclinone - calbistrin), suggesting these compounds are substrates for the yeast $P D R$ efflux network pumps. A table of reported activities and/or mechanisms-of-action for the 32 biologically active compounds can be found in appendix 1.1 of this thesis. Two compounds from this screen, tetramic acid 289 (TA-289), a novel biologically active metabolite isolated by Dr Stephen Bloor, and equisetin, a known, structurally-related tetramic-acid compound with an uncharacterized mode-of-action, were chosen for further drugtarget identification studies. For this project, both compounds were chosen due to their unknown modes-of-action, and also because greater quantities were more readily available for study.

\subsubsection{TA-289 and equisetin display pH-dependent cytotoxic activities}

To determine the MIC concentrations of TA-289 and equisetin, dose-response assays in both liquid and solid media were performed. It was first observed that both TA289 and equisetin varied in potency under different media conditions. To determine whether the bioactivities of both compounds displayed media-dependent or $\mathrm{pH}$ dependent growth inhibitory activities, liquid dose-response assays were performed in two standard media types at low and high pH levels; SC (normal pH 4.0), SC-H (buffered to $\mathrm{pH} 8.0$ ), YPD (normal $\mathrm{pH} ~ 7.0$ ), and YPD-HCl (buffered to $\mathrm{pH} 4.0$ ). 
Under unbuffered conditions (SC, $\mathrm{pH} 4.0$ ), TA-289 and equisetin displayed potent growth inhibitory activities in both yeast strains tested at micromolar concentrations. When the compounds were tested in media at higher $\mathrm{pH}(\mathrm{pH} 8.0$ SC-H, and $\mathrm{pH}$ 7.0 YPD), this activity was abrogated completely for TA-289, and partially for equisetin (Figure 2.4, appendix 1.2). Interestingly, activities of TA-289 and equisetin were observed in $\mathrm{pH}$ 4.0 YPD-HCl, strongly indicating that TA-289 and equisetin display $\mathrm{pH}$-dependent growth inhibitory activities, and not because of the media type used (i.e. synthetic amino acid mix vs. yeast extract).

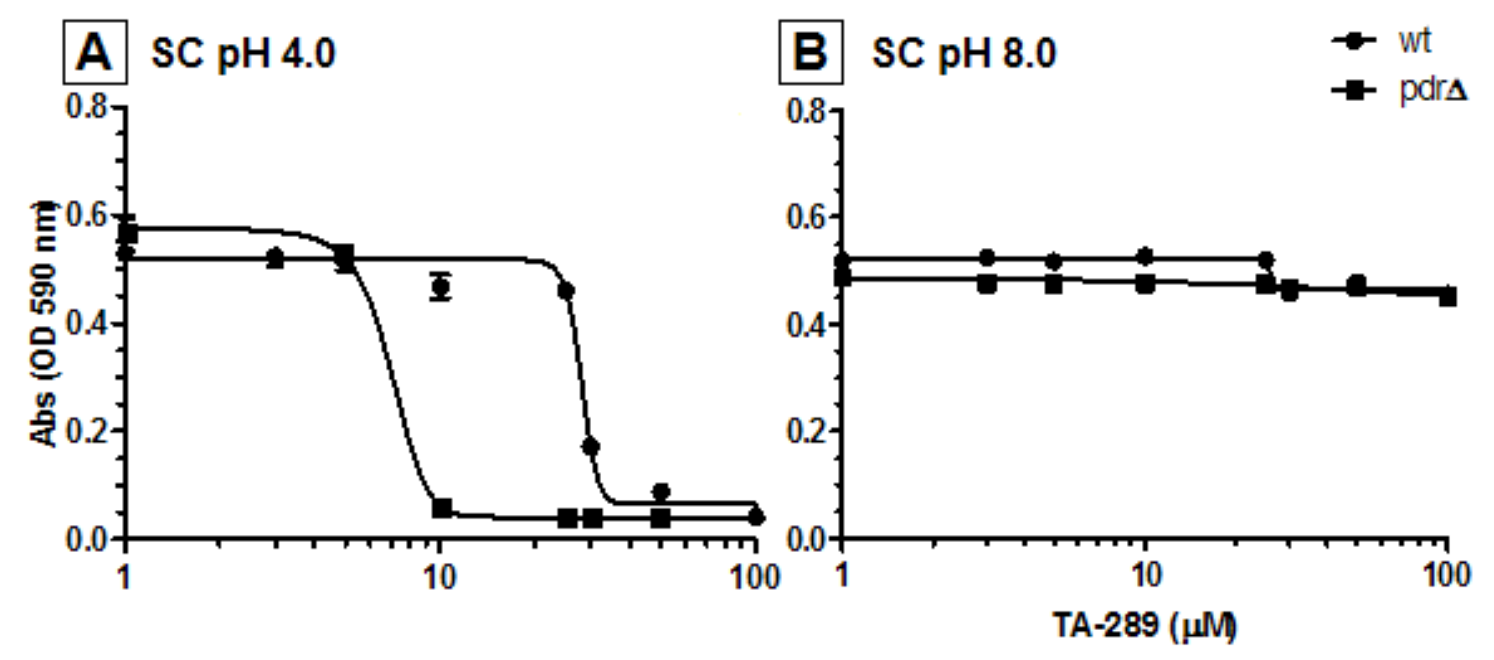

Figure 2.4. Growth-inhibitory effects of TA-289 are pH-dependent. wt (circles) and pdr $\Delta$ (squares) cells were treated with varying concentrations of TA-289 (x-axes) for $18 \mathrm{~h}$ in SC at two different $\mathrm{pH}$ conditions; $\mathrm{pH} 4.0$ (A) and $\mathrm{pH} 8.0$ (B). Cell density was measured by absorbance at OD $590 \mathrm{~nm}$ (y-axes). See appendix 1.2 for all dose-response assays.

MIC values were calculated for each compound on the two yeast strains tested, as well as under the four different media conditions (Table 2.1). TA-289 and equisetin also displayed differential activity against BY4742 and pdrA strains, where a threefold difference in potency of both compounds was observed between BY4742 and 
the $p d r \Delta$ strain. This suggests that both TA-289 and equisetin are substrates for the drug-efflux pump system in yeast. Notably, these differential growth inhibitory activities were not observed in the initial growth inhibition assays (Figure 2.3), where both compounds potently inhibited growth of both the BY4742 and pdrA strain at $100 \mu \mathrm{M}$.

Table 2.1. MIC values of TA-289 and equisetin in wt and pdrA strains under different media conditions.

\begin{tabular}{|l|c|c|c|c|}
\hline \multirow{2}{*}{ Media } & \multicolumn{2}{|c|}{ MIC $(\mu \mathrm{M})$ TA-289 } & \multicolumn{2}{c|}{ MIC $(\mu \mathrm{M})$ Equisetin } \\
\cline { 2 - 5 } & $\mathrm{wt}$ & $p d r \Delta$ & $\mathrm{wt}$ & $p d r \Delta$ \\
\hline SC (pH 4.0) & 50 & 10 & 30 & 25 \\
\hline SC-H (pH 8.0) & $>100$ & $>100$ & 50 & 50 \\
\hline YPD-HCl (pH 4.0) & 50 & 25 & 30 & 25 \\
\hline YPD (pH 7.0) & $>100$ & $>100$ & $>100$ & 50 \\
\hline
\end{tabular}

Solid media dose-response assays were conducted in $\mathrm{pH} 4.0 \mathrm{SC}$, the $\mathrm{pH}$ condition where TA-289 and equisetin displayed strongest potency in yeast. The assays revealed the minimum inhibitory concentration (MIC) value for both compounds for future experiments conducted using solid media. The wt strain was not growth inhibited by either TA-289 or equisetin at any concentration tested. The pdr $\Delta$ strain however, did not grow in $50 \mu \mathrm{M}$ TA-289 and equisetin, thus determining the MIC in solid media for both TA-289 and equisetin to be $50 \mu \mathrm{M}$ in the $p d r \Delta$ strain (Figure 2.5). 


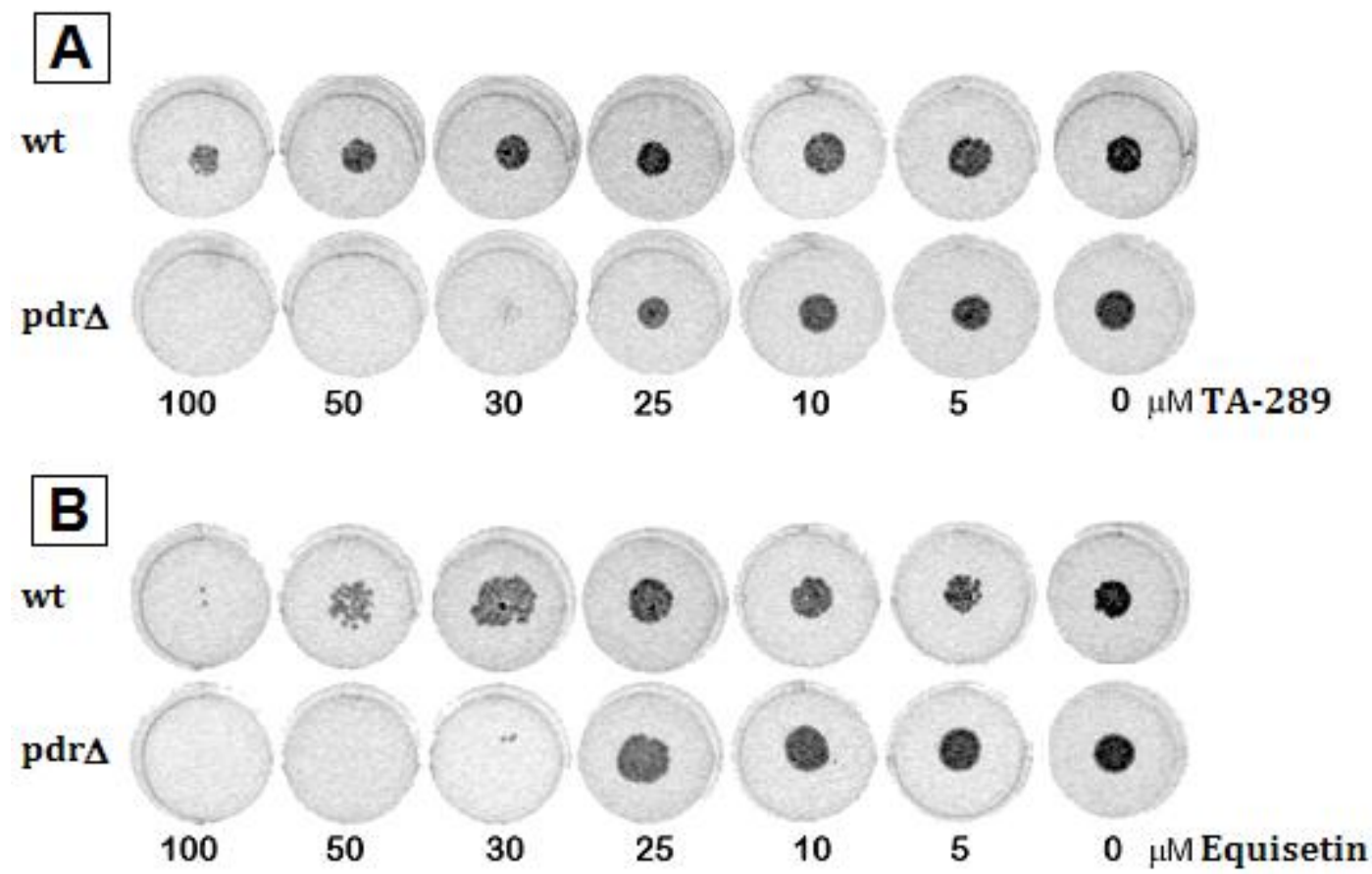

Figure 2.5. Solid dose-response assay of TA-289 and equisetin. SC media containing varying concentrations of TA-289 and equisetin were inoculated with $5 \times 10^{4}$ cells per well and incubated for 2 days at $30{ }^{\circ} \mathrm{C}$. Bioactivities of TA-289 (A) and equisetin (B) were assessed by observing colony growth of wt (BY4742) and $p d r \Delta$ compared to the respective untreated controls.

Additionally, to determine whether the bioactivities exhibited by these compounds were cytotoxic i.e. cause cell death or irreversible growth inhibition, or cytostatic (reversible), a colony forming assay was performed and the ability of cells to recover from drug treatment was assessed. Interestingly, both TA-289 and equisetin displayed cytotoxic, or at least irreversible growth inhibitory effects in only the $p d r \Delta$ cells, where the cells washed of the compounds were unable to recover after two days of incubation, compared to both DMSO- and cycloheximide-treated cells (Figure 2.6). This was not observed in BY4742 (wt) cells, and especially in wt cells 
treated with TA-289 which recovered just as well as the negative controls for cell death (DMSO and cycloheximide, which do not kill yeast). In wt cells that were treated with equisetin, cell recovery was slower and much less, however still present. This may be attributed to equisetin being more potent in yeast than TA289.

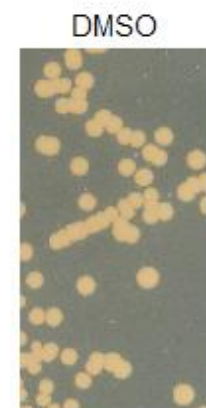

$(-)$

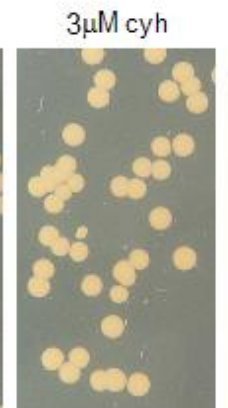

$(-)$

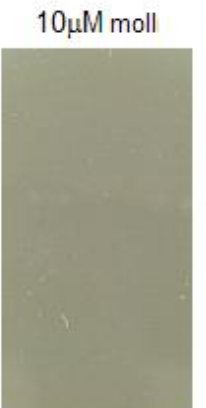

$(+)$

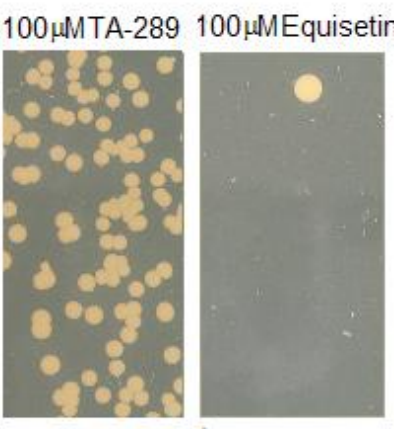

wt

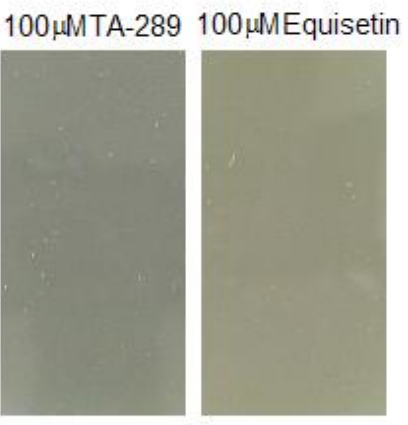

$\operatorname{pdr} \Delta$

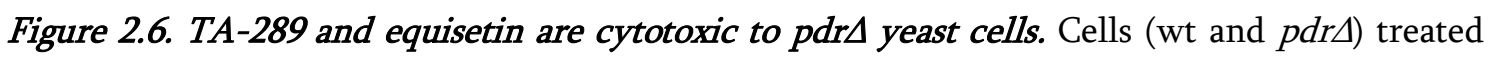
overnight with either $100 \mu \mathrm{M}$ TA-289, equisetin, $3 \mu \mathrm{M}$ cycloheximide (cyh), or $10 \mu \mathrm{M}$ mollisoside, were washed twice with $\mathrm{ddH}_{2} \mathrm{O}$, diluted, and aliquots were plated onto rich media petri plates to give a final concentration of 30 cells per plate, after which plates were incubated for 2 days at $30{ }^{\circ} \mathrm{C}$ and then cell growth observed. Cell growth was also compared to untreated cells (DMSO), and mollisoside, a positive control for cell death.

\subsubsection{Cell morphology}

To determine whether TA-289 caused gross morphological changes in yeast, TA289-treated cells were observed under a microscope and cell morphology manually examined. At the highest concentration of TA-289 $(100 \mu \mathrm{M})$, both wt and pdr $\Delta$ cells were statistically significantly smaller in size compared to untreated controls (Table 2.2, Figure 2.7), with the average cell size being 1.67 fold smaller than untreated cells. Cells treated with a lower concentration, $30 \mu \mathrm{M}$, were also smaller, at 1.27 fold 
smaller than untreated control cells. After treatment, similar phenotypes were observed in $p d r \Delta$ cells. See appendix 1.3 for photographs of $p d r \Delta$ cells. Aside from cell size, no gross morphological changes were observed.

Table 2.2. Cell sizes of wt and pdrA cells treated with TA-289. Cells were treated with either $30 \mu \mathrm{M}$ or $100 \mu \mathrm{M}$ TA-289 for $18 \mathrm{~h}$ and size of cells were measured. An un-paired student t-test was performed, and each value represents the fold decrease in cell size compared to untreated control cells. The corresponding $\mathrm{p}$-values are indicated for each treatment.

\begin{tabular}{|c|c|c|}
\hline Strain & wt & $p d r \Delta$ \\
\hline $30 \mu \mathrm{M}$ TA-289 & $1.27\left(\mathrm{p}=2.16 \times 10^{-6}\right)$ & $1.15\left(\mathrm{p}=1.15 \times 10^{-4}\right)$ \\
\hline $100 \mu \mathrm{M}$ TA-289 & $1.67\left(\mathrm{p}=3.24 \times 10^{-26}\right)$ & $1.41\left(\mathrm{p}=2.67 \times 10^{-16}\right)$ \\
\hline
\end{tabular}

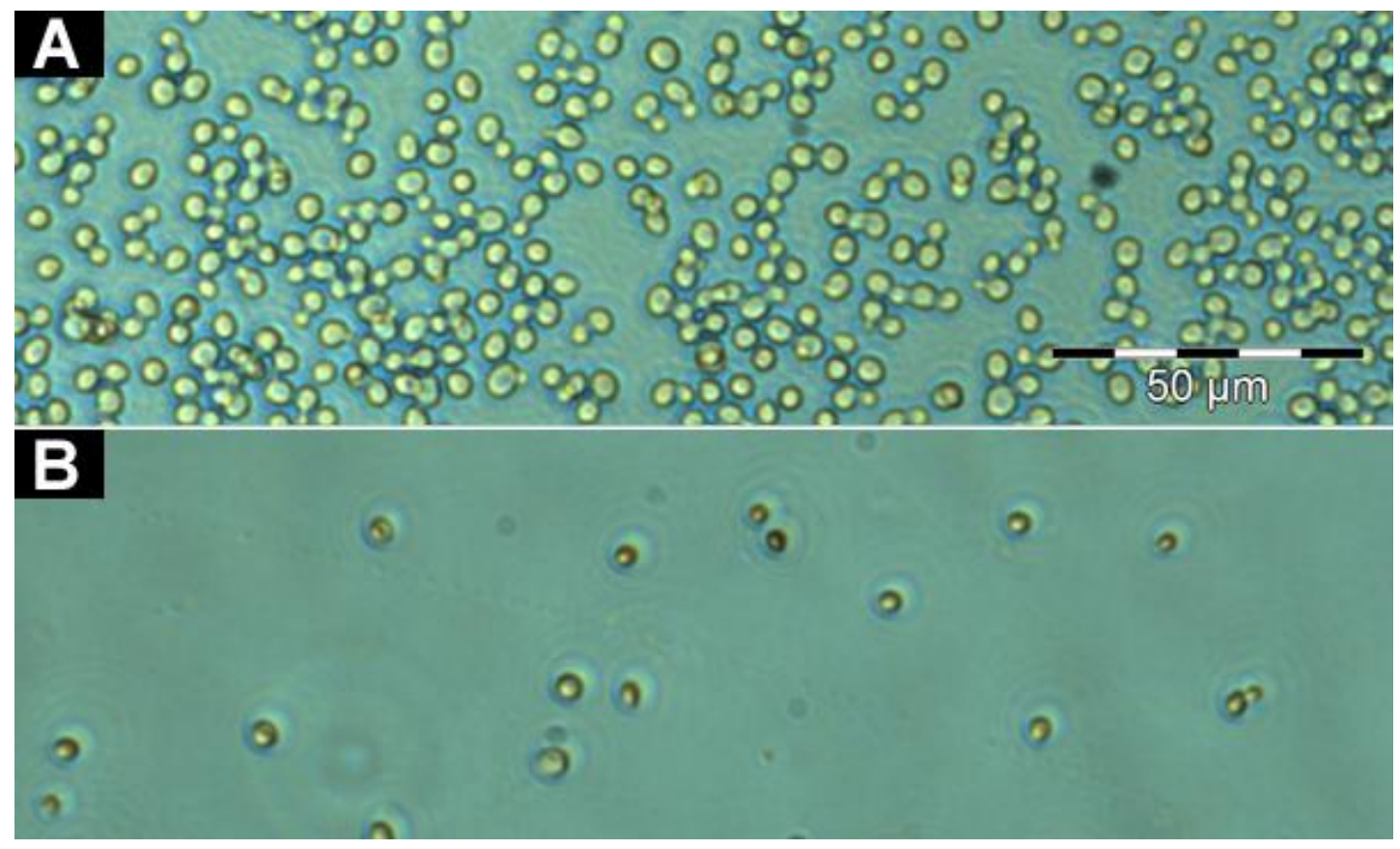

Figure 2.7. TA-289 causes a small-size phenotype in yeast. TA-289-treated (100 $\mu \mathrm{M})(\mathrm{B})$ wt cells display a small-size phenotype after $18 \mathrm{~h}$ treatment compared to DMSO control cells (A). See appendix 1.3 for all photographs. 
The presence of cells at the highest concentration of TA-289 and equisetin tested indicate that cells may be irreversibly growth inhibited (if yeast cells do not bleb and "disappear" during apoptosis (as that observed in mammalian apoptosis (Madeo, Carmona-Gutierrez et al. 2009)), it is difficult to distinguish between dead cells and irreversibly growth inhibited cells), especially in the case of $p d r \Delta$ cells,

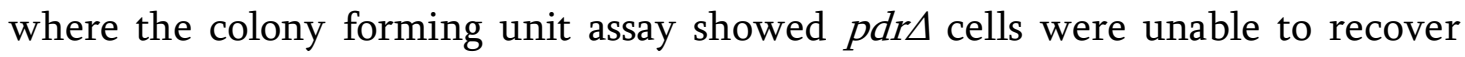
from treatment with TA-289 or equisetin. Likewise, flow cytometric analysis of treated cells taken from the end point of liquid dose-response assays compared with a control yeGFP population of the same starting cell concentration used for a liquid dose-response assay also showed the presence of cells (Figure 2.8). A higher percentage of non-yeGFP cells in the BY4742-treated samples may indicate that BY4742 cells were slowly recovering from treatment with TA-289 and equisetin. This may just be a sub-population of cells that were able to survive treatment with TA-289 and equisetin and are thus slowly growing. The larger percentage of recovering wt cells compared to $p d r \Delta$ cells is further evidence for TA-289 and equisetin being substrates for the yeast efflux pumps, where wt cells are able to actively expel TA-289 and equisetin from within the cell to aid in the recovery process. 


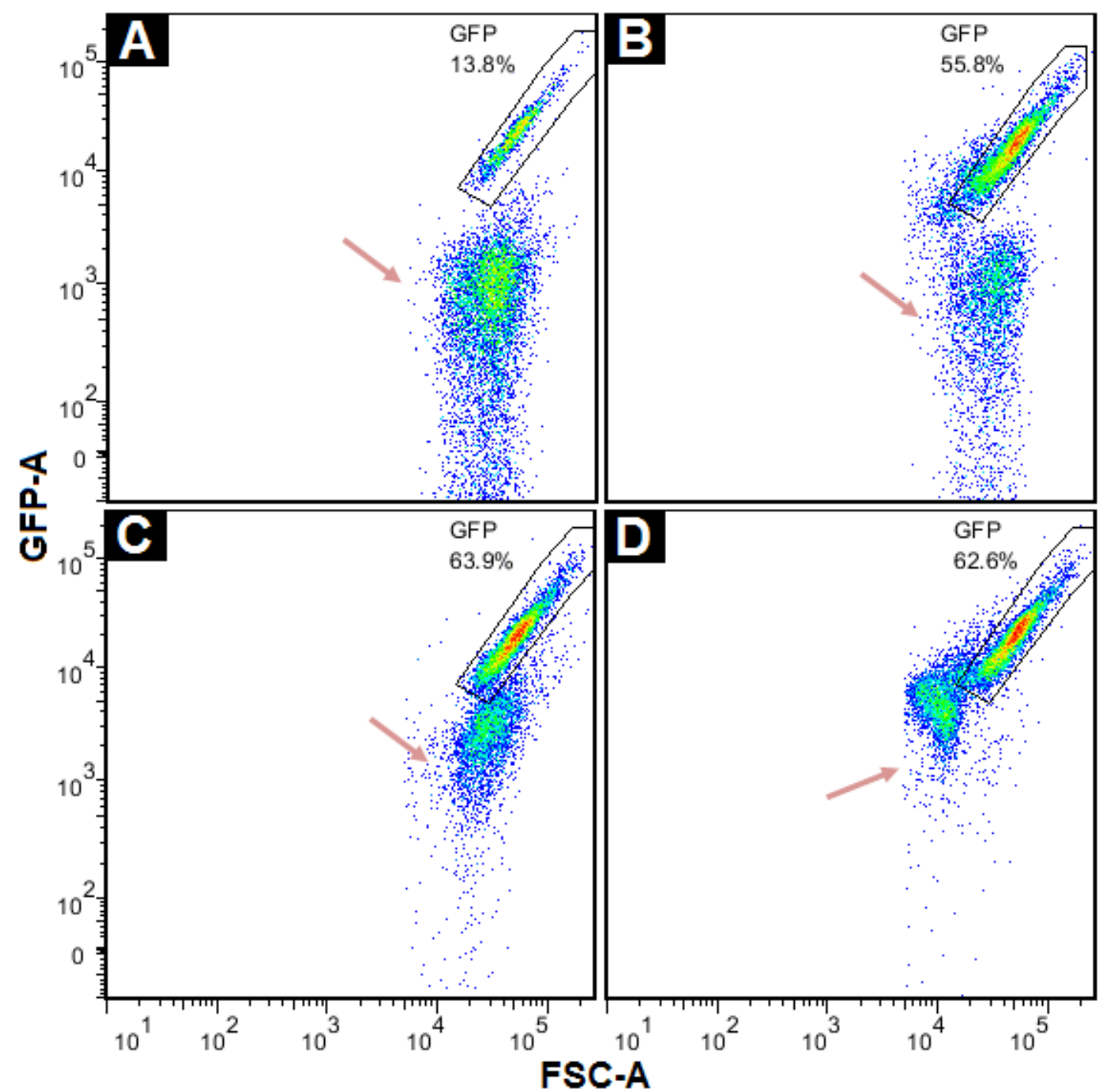

Figure 2.8. Cells are present after 18 h treatment with TA-289 or equisetin. BY4742 (wt) cells treated with $100 \mu \mathrm{M}$ TA-289 (A), equisetin (B), and $p d r \Delta$ cells treated with TA-289 (C), and equisetin (D), taken from the end point of a liquid dose-response assay were mixed with a population of yeGFP cells and visualized by flow cytometry. 


\subsubsection{TA-289 and equisetin cause late phase cell cycle block}

Flow cytometric analysis of yeast DNA content was performed to determine whether TA-289 and equisetin caused a cell cycle block at a given cell cycle stage. Cells treated with either TA-289 and equisetin revealed a decrease in the population of cells at in $\mathrm{G}_{1}$ phase, and an accumulation of cells at the later phases of the cell cycle, namely at S-G2/M phase, compared to untreated controls - this was especially so for $p d r \Delta$ cells treated with TA-289 and equisetin (Figure 2.9, Table 2.3). The observed accumulation of treated cells indicates TA-289 and equisetin inhibit growth of yeast by causing a cell cycle block at $\mathrm{S}-\mathrm{G}_{2} / \mathrm{M}$ phase.

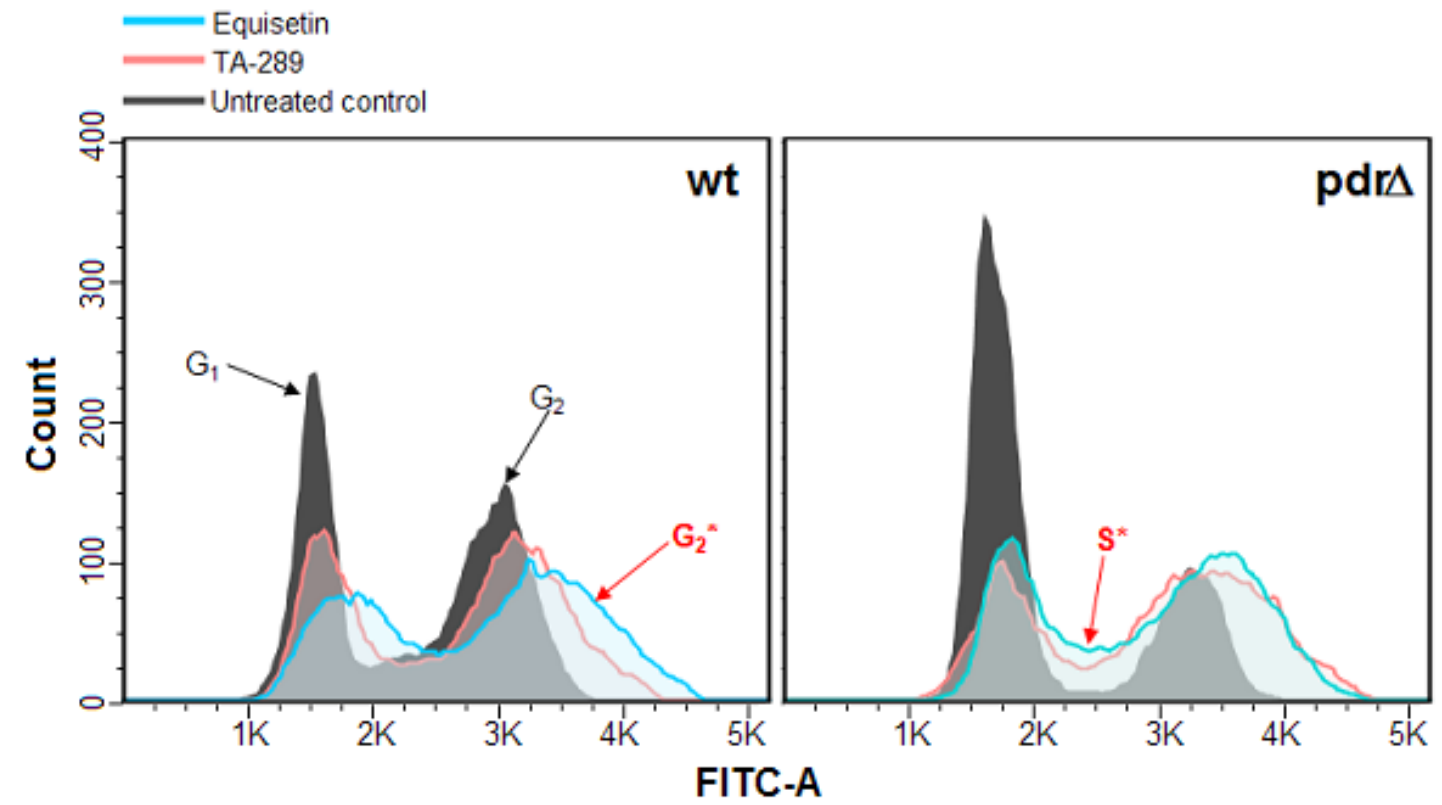

Figure 2.9. TA-289 and equisetin cause late-phase cell cycle block. BY4742 (wt) and pdrA cells treated with $100 \mu \mathrm{M}$ TA-289 or equisetin for $4 \mathrm{~h}$ were fixed and stained with SYTOX green, and subsequently visualized by flow cytometry. Histogram overlays show wt and $p d r \Delta$ untreated controls (black) compared with cells treated with equisetin (blue) and TA-289 (pink). Untreated control $\mathrm{G}_{1}$ and $\mathrm{G}_{2} / \mathrm{M}\left(\mathrm{G}_{2}\right)$ phases are indicated in black. Changes in $\mathrm{S}_{\text {and }} \mathrm{G}_{2} / \mathrm{M}$ phase of treated cells are indicated in red. 


\begin{tabular}{|c|c|c|c|c|c|c|c|c|c|}
\hline & \multicolumn{9}{|c|}{ wt } \\
\hline & \multicolumn{3}{|c|}{ TA-289 $(100 \mu \mathrm{M})$} & \multicolumn{3}{|c|}{ Equisetin $(100 \mu \mathrm{M})$} & \multicolumn{3}{|c|}{ Untreated (-) } \\
\hline $\begin{array}{l}\text { Time } \\
\text { (mins) }\end{array}$ & $\mathrm{G}_{1}$ & $\mathrm{~S}$ & $\mathrm{G}_{2} / \mathrm{M}$ & $\mathrm{G}_{1}$ & $\mathrm{~S}$ & $\mathrm{G}_{2} / \mathrm{M}$ & $\mathrm{G}_{1}$ & $\mathrm{~S}$ & $\mathrm{G}_{2} / \mathrm{M}$ \\
\hline 0 & 31 & 17 & 43 & 28 & 19 & 46 & 40 & 9 & 46 \\
\hline 30 & 42 & 16 & 38 & 34 & 17 & 42 & 52 & 9 & 35 \\
\hline 60 & 39 & 16 & 39 & 34 & 17 & 41 & 38 & 10 & 45 \\
\hline 90 & 37 & 16 & 44 & 36 & 16 & 41 & 35 & 11 & 45 \\
\hline & \multicolumn{9}{|c|}{$\operatorname{pdr} \Delta$} \\
\hline & \multicolumn{3}{|c|}{ TA-289 $(100 \mu \mathrm{M})$} & \multicolumn{3}{|c|}{ Equisetin $(100 \mu \mathrm{M})$} & \multicolumn{3}{|c|}{ Untreated (-) } \\
\hline $\begin{array}{l}\text { Time } \\
\text { (mins) }\end{array}$ & $\mathrm{G}_{1}$ & S & $\mathrm{G}_{2} / \mathrm{M}$ & $\mathrm{G}_{1}$ & $\mathrm{~S}$ & $\mathrm{G}_{2} / \mathrm{M}$ & $\mathrm{G}_{1}$ & $\mathrm{~S}$ & $\mathrm{G}_{2} / \mathrm{M}$ \\
\hline 0 & 26 & $\sqrt{16}$ & 47 & 28 & $\overline{17}$ & 49 & 68 & 4 & 25 \\
\hline 30 & 30 & 17 & 50 & 30 & 21 & 45 & 52 & 13 & 30 \\
\hline 60 & 28 & 17 & 50 & 30 & 18 & 49 & 39 & 11 & 47 \\
\hline 90 & 26 & 18 & 50 & 33 & 19 & 44 & 40 & 13 & 44 \\
\hline
\end{tabular}

Table 2.3. Percentage of both wt and pdr $\Delta$ cells in $G_{1}, S$, and $G_{2} / M$ phase of the cell cycle when treated with $100 \mu \mathrm{M}$ TA-289 or equisetin compared to untreated controls. Time (mins) indicate the time after washing cells after a $4 \mathrm{~h}$ treatment with TA-289 and equisetin. All values represented as percentages of the total gated population. Highlighted in blue are observed accumulation of $\mathrm{S}$ and $\mathrm{G} 2 / \mathrm{M}$ phase in TA-289- and equisetin-treated cells.

A time-course analysis showed that $p d r \Delta$ cells were unable to recover to a normal cell cycle distribution following withdrawal of both TA-289 and equisetin after 1.5 h, while wt cells recovered from treatment with TA-289 within 30 mins after washing, and slower recovery in equisetin (over $1 \mathrm{~h}$ after washing). These cell recovery results replicate what was observed in the colony forming unit assay (Figure 2.6), wherein wt cells treated in equisetin displayed slower recovery rates 
than wt cells treated with TA-289, while pdr $\Delta$ cells did not recover from treatment of either compounds (Figure 2.10). See appendix 1.4 for equisetin time-course histograms.

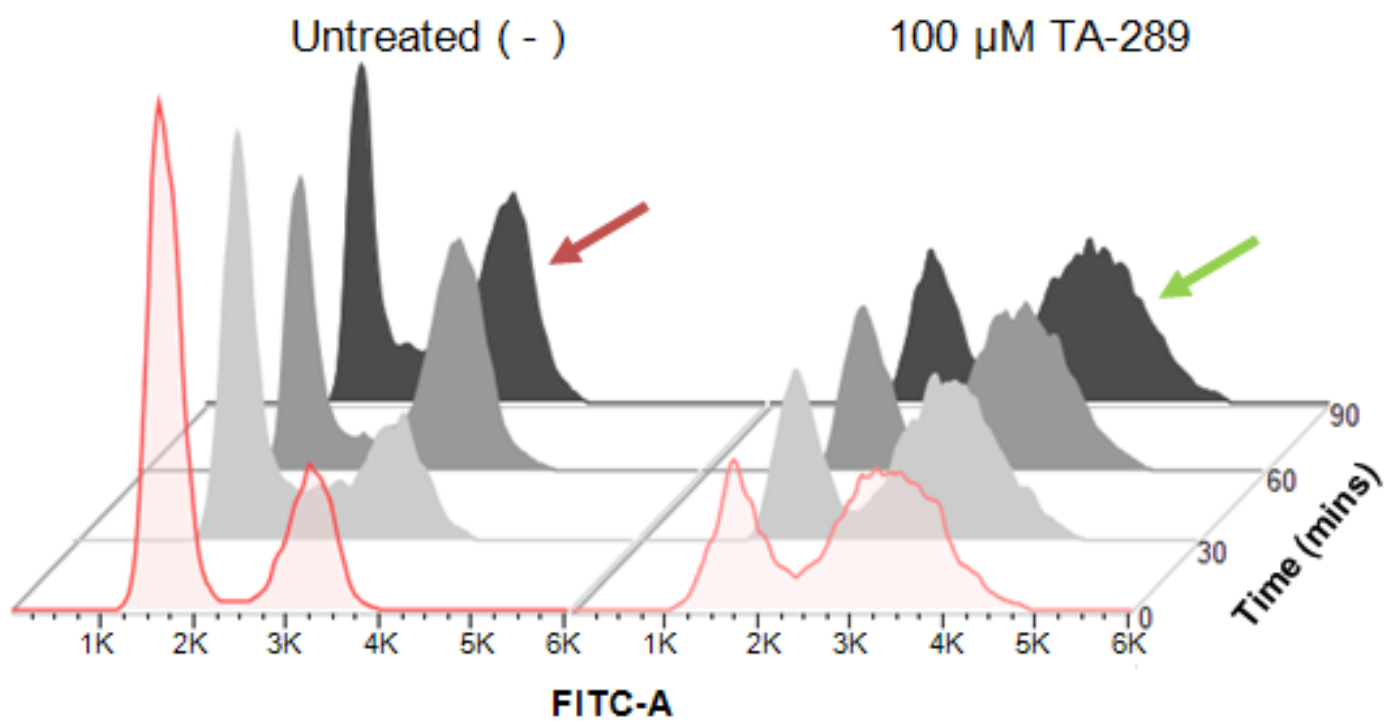

Figure 2.10. TA-289 causes irreversible cell cycle block in pdrA cells. Cells treated with either TA-289 or equisetin were washed after a $4 \mathrm{~h}$ incubation period. Cells were then harvested every 30 mins for $1.5 \mathrm{~h}$ and visualised by flow cytometry. Above shows an example of a time course histogram overlay of $p d r \Delta$ cells treated with TA-289 compared to their corresponding controls. Red arrow highlights the increase in $\mathrm{G}_{2} / \mathrm{M}$ phase from untreated cells growing normally, however this was not observed in cells treated with $100 \mu \mathrm{M}$ TA-289, which were were unable to recover from treatment 90 mins after washing (green arrow). See appendix 1.4 for all time course histograms.

Often, a cell cycle block at $\mathrm{G}_{2} / \mathrm{M}$ phase is indicative of a tubulin-targeting compound that prevents progression through mitosis (Hamel 1996). To determine whether TA289 or equisetin target tubulin, the $t u b 2 \Delta:: \operatorname{Kan} R$ heterozygous diploid was tested for sensitivity to both compounds. No differential sensitivity was observed between the tub2 $\Delta$ strain and the diploid BY4743 strain when treated with TA-289 or equisetin, 
as compared to hypersensitivity of the tub2 strain to the anti-mitotic agent benomyl (Thomas, Neff et al. 1985), suggesting that TA-289 and equisetin are not tubulin-targeting compounds (Figure 2.11).
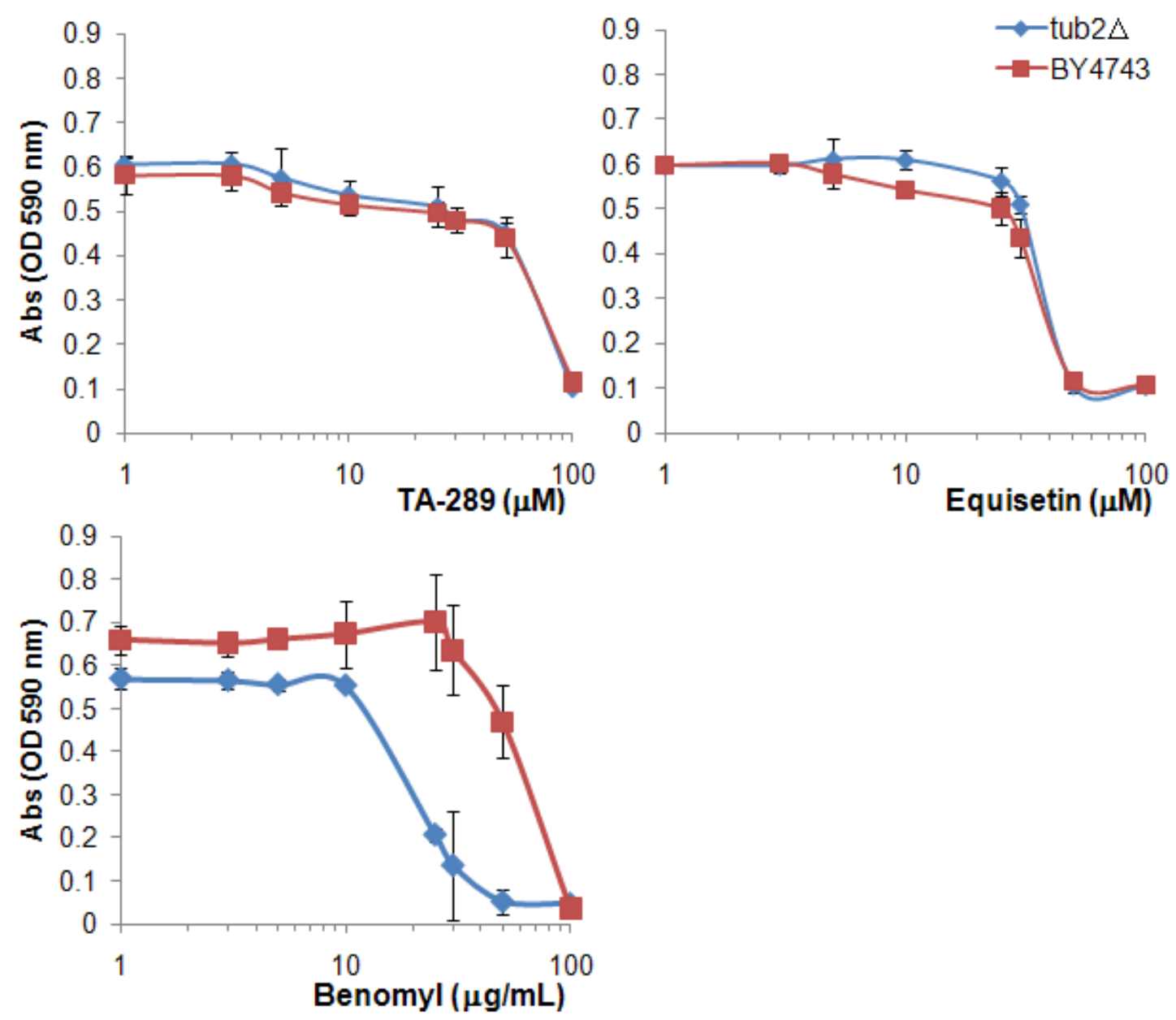

Figure 2.11. TA-289 and equisetin do not target tubulin. tub2 $\triangle$ and wt diploid (BY4743) cells treated with a serial dilution of either TA-289 or equisetin, incubated for $18 \mathrm{~h}$ at $30 \mathrm{C}$ and cell growth measured by determining OD at $590 \mathrm{~nm}$. 


\subsubsection{Chemical genomic profiling of TA-289}

Chemical genomic profiles of TA-289 were generated by performing a yeast tag gDNA microarray utilizing two yeast deletion sets, the heterozygous diploid deletion set (HET) and the homozygous diploid deletion set (HOM). These deletion pools were treated with $10 \mu \mathrm{M}$ TA-289 (a concentration that causes approximately 30\% growth inhibition) used to probe affected biological pathways most likely required to buffer the inhibitory effects of TA-289. A total of 4466 genes (after filtering and normalization of data, including up and down tags, with replicates removed) were identified in the first HOM screen, and 4021 genes in the second HOM screen. An overlap of 4016 genes was identified between the two HOM screens. A total of 5236 genes were identified in the HET screen, of which 4103 genes overlapped with the two HOM screens. A list of the top 40 sensitive gene hits from the HOM profiles (top 20 from each HOM screen), were categorized according to the biological processes from which they are involved (Table 2.4). These were selected by removing genes first identified to be multi-drug resistance genes according to a previously published large-scale chemical genomic screen data set (Hillenmeyer, Fung et al. 2008), and then filtering by deviation scores, where a D (deviation)-score of $<-3$ is deemed dubious. 


\begin{tabular}{|c|c|c|c|c|}
\hline ORF & Name & D-score & Component & Biological process \\
\hline YHR124W & NDT80 & -3.00 & Nuc & Cell cycle \\
\hline YDR123C & INO2 & -2.99 & Nuc & Phospholipid biosynthesis \\
\hline YNL097C & PHO23 & -2.97 & Nuc & Phosphate metabolism \\
\hline YPL213W & LEA1 & -2.96 & Cyt & U2 snRNP \\
\hline YNL059C & ARP5 & -2.94 & Nuc & Actin, chromatin remodeling \\
\hline YLR370C & ARC18 & -2.93 & Mito & Actin, mitochondrion localisation \\
\hline YHR154W & RTT107 & -2.93 & Nuc & DNA repair, cell cycle \\
\hline YKR007W & YKR007W & -2.92 & Endo & Microautophagy \\
\hline YJR118C & ILM1 & -2.91 & ER & Unknown, mitochondrial DNA maintenance \\
\hline YDR207C & UME6 & -2.87 & Nuc & Cell cycle \\
\hline YDR268W & MSW1 & -2.87 & Mito & Mitochondrial tryptophanyl-tRNA synthetase \\
\hline YHR021C & RPS27B & -2.86 & Cyt & Ribosomal subunit \\
\hline YEL061C & CIN8 & -2.82 & Mito & Cell cycle \\
\hline YBR194W & YBR194W & -2.81 & Cyt & Nuclear pore complex \\
\hline YHR178W & STB5 & -2.75 & Nuc & Multidrug resistance, oxidative stress response \\
\hline YGL254W & SNF4 & -2.75 & Cyt & Glucose-repression, peroxisome biogenesis \\
\hline YDL020C & RPN4 & -2.70 & Nuc & Cell cycle, stress response \\
\hline YJR033C & RAV1 & -2.69 & Cyt & Endosome \\
\hline YHL008C & FZF1 & -2.69 & Nuc & Sulfite metabolism \\
\hline YBR170C & NPL4 & -2.65 & ER & ER and mitochondrial degradation \\
\hline YJR100C & YJR100C & -2.65 & Mito & Unknown, mitochondrial genome loss \\
\hline YBR278W & DPB3 & -2.65 & Nuc & DNA polymerase II \\
\hline YMR097C & MTG1 & -2.64 & Mito & GTPase essential for respiration \\
\hline YML121W & GTR1 & -2.63 & Cyt, Vac & GTP binding protein, phosphate transport \\
\hline YNL297C & MON2 & -2.62 & Endo & Endocytosis \\
\hline YGL215W & CLG1 & -2.53 & N/A & Autophagy, Cell cycle \\
\hline YLR201C & YLR201C & -2.52 & Mito & $\begin{array}{l}\text { Ubiquinone biosynthesis, mitochondrial inner } \\
\text { membrane }\end{array}$ \\
\hline YJL082W & IML2 & -2.51 & Mito & Unknown, mitochondrial DNA maintenance \\
\hline YOL051W & GAL11 & -2.49 & N/A & RNA polymerase II mediator complex \\
\hline YKL190W & $\mathrm{CNB} 1$ & -2.48 & Calcineurin & $\begin{array}{l}\text { Regulates stress response transcription factor } \\
\text { Crz1p }\end{array}$ \\
\hline YDR438W & YDR438W & -2.46 & Mito & Mitochondrial transporter \\
\hline YPR199C & ARR1 & -2.40 & Nuc, Cyt & Transcriptional activator of bZIP \\
\hline YGL198W & YIP4 & -2.39 & Golgi & Vesicular transport \\
\hline YEL042W & GDA1 & -2.39 & Golgi & Guanosine diphosphatase \\
\hline YCL014W & BUD3 & -2.37 & Bud neck & Cell cycle \\
\hline YOL004W & SIN3 & -2.36 & N/A & Histone deacetylase \\
\hline YBR216C & YBP1 & -2.35 & Cyt & Stress response, Yap1p binding protein \\
\hline YOR330C & MIP1 & -2.34 & Mito & Mitochondrial DNA polymerase \\
\hline YBR231C & AOR1 & -2.30 & Nuc & Unknown \\
\hline YEL060C & PRB1 & -2.24 & Vac & $\begin{array}{l}\text { Vacuolar protein degradation, response to } \\
\text { starvation }\end{array}$ \\
\hline
\end{tabular}

Table 2.4. List of top 40 hypersensitive hits from HOM screens. Genes were grouped according to biological processes, and ordered according to sensitivity to TA-289, where the most negative D-score value indicates greatest sensitivity. Highlighted in blue are genes shown to be localized to the mitochondrion or the ER, and/or are involved in mitochondrial-associated processes. See supplementary file 1 for the complete list of genes identified as sensitive or resistant, their assigned sensitivity scores and associated biological processes. Key: Nuc $=$ Nucleus, Cyt $=$ Cytoplasm, Mito $=$ Mitochondrion, $\mathrm{Vac}=$ Vacuole, Endo $=$ Endosome, $\mathrm{ER}=$ Endoplasmic reticulum, Golgi $=$ Golgi apparatus. 
Of these, 11 were either involved in ER and mitochondrial processes or were localized to the mitochondrion or the ER. Previously described studies on equisetin showed the compound having effects on isolated mitochondria (König, Kapus et al. 1993) and the ER (Patham, Duffy et al. 2009). Due to their structural similarities, and the enrichment of mitochondria and ER genes identified in these chemical genomic screens for TA-289, TA-289 may share the same mechanism-of-action with equisetin. Genes associated with oxidative stress responses and DNA repair, and cell cycle-related genes were also identified, most of which were shown to be involved in mitosis and cytokinesis (CIN8, RPN4, and BUD3) which reflects on the late phase cell cycle block observed in the cell cycle analyses performed using flow cytometry (Figure 2.9).

A list of the top 15 sensitive gene hits from the HET screen is displayed below. These were selected again by removing the multi-drug resistance genes as described previously, however the most sensitive genes were selected, following the idea of haplo-insufficiency where the heterozygote strain containing the target protein will be hypersensitive (Table 2.5). 


\begin{tabular}{|c|c|c|c|c|c|}
\hline ORF & Name & D-scores & Essential & Component & Description \\
\hline YER112W & LSM4 & -3.71 & 1 & Nuc & mRNA decay \\
\hline YOR174W & MED4 & -3.70 & 1 & N/A & $\begin{array}{l}\text { Subunit of RNA polymerase } \\
\text { II mediator complex } \\
\text { essential for translation }\end{array}$ \\
\hline YPR065W & ROX1 & -3.61 & 0 & Nuc & $\begin{array}{l}\text { Heme-dependent repressor } \\
\text { of hypoxic genes }\end{array}$ \\
\hline YIL016W & SNL1 & -3.36 & 0 & Mito, ER & Unknown \\
\hline YIR025W & MND2 & -3.35 & 0 & Nuc & $\begin{array}{l}\text { Subunit of anaphase- } \\
\text { promoting complex }\end{array}$ \\
\hline YBL078C & AUT7 & -3.28 & 0 & Vac & Autophagy, mitophagy \\
\hline YDL238C & YDL238C & -3.26 & 0 & Cyt & Guanine deaminase \\
\hline YIL036W & CST6 & -3.24 & 0 & Nuc & $\begin{array}{l}\text { Leucine zipper } \\
\text { transcription factor, } \\
\text { chromosome stability }\end{array}$ \\
\hline YJL154C & VPS35 & -3.19 & 0 & Endo & $\begin{array}{l}\text { Retromer complex, } \\
\text { retrograde transport }\end{array}$ \\
\hline YNL077W & APJ1 & -3.13 & 0 & Mito, Cyt & $\begin{array}{l}\text { Putative chaperone of } \\
\text { HSP } 40\end{array}$ \\
\hline YNL131W & TOM22 & -3.06 & 1 & Mito & $\begin{array}{l}\text { Component of TOM, } \\
\text { responsible for import of } \\
\text { mitochondrially-directed } \\
\text { proteins }\end{array}$ \\
\hline YBR248C & HIS7 & -2.99 & 0 & N/A & $\begin{array}{l}\text { Imidazole glycerol } \\
\text { phosphate synthase }\end{array}$ \\
\hline YBR038W & CHS2 & -2.98 & 1 & Bud neck & $\begin{array}{l}\text { Chitin synthase II, cell } \\
\text { cycle }\end{array}$ \\
\hline YDL106C & $\mathrm{PHO} 2$ & -2.96 & 0 & Nuc & Phosphate metabolism \\
\hline
\end{tabular}

Table 2.5. List of top 15 hypersensitive hits from the HET screen. Genes were grouped according to biological processes, and ordered according to sensitivity to TA-289, where the most negative D-score value indicates greatest sensitivity. Essential genes are indicated with a value of 1, nonessentials with a value of 0 . Highlighted in blue are genes of interest, previously described to be involved in mitochondrial-related processes. See supplementary file 1 for the complete list of genes identified as sensitive or resistant, their assigned sensitivity scores and associated biological processes. Key: Nuc $=$ Nucleus, Cyt $=$ Cytoplasm, Mito $=$ Mitochondrion, Vac $=$ Vacuole, Endo $=$ Endosome, ER = endoplasmic reticulum. 
Of these 15, four were identified as essential genes, including TOM22, which plays regulatory roles in mitochondrial protein import. AUT7 has also been previously implicated in mitochondrial maintenance and degradation (i.e. mitophagy), of which the null mutant displayed abnormal mitochondrial function (Zhang, Qi et al. 2007).

Moreover, mitochondrial electron transport chain (ETC) associated genes (complexes I through V) were also identified as sensitive in both HET and HOM screens. These included NADH dehydrogenase and NADH ubiquinone oxireductase $N D E 1$ and 2, and NDI1, succinate dehydrogenase SDH2, 3, and 4, bc1 complex (cytochrome $c$ reductase) $Q C R 2,6,7,9,10$, and $C Y T 1$ and 2, ubiquinone/coenzyme Q COQ1, 3, 6, and 9 (identified in the top 40 hypersensitive genes as YLR201C in List 2.1) cytochrome c oxidase $C O X 5 A, 5 B, 7,8,9,11,14,15,16,18,19$ and $20, P E T$ (required for respiratory growth; ADP/ATP carrier, mitochondria ribosomal subunit, mitochondrial translational activator) $9,10,18,54,56,100,111,112,123,127,130$, 191, and 309, and F0-F1 ATP synthase ATP2, 3, 5, 10, 11, 16, 18, and 20 (see supplementary file 1). These results are consistent with previous indications of equisetin eliciting effects with mitochondria- and ER-related processes, and is also evidence for TA-289 exhibiting a similar mechanism.

Hierarchical cluster analyses performed on datasets from both HOM screens with a previously published large-scale chemical genomic screen dataset (Hillenmeyer, 
Fung et al. 2008) revealed similarity between the profile of TA-289 and those of Ldopa and MPP+, i.e. L-dopa and MPP+ branched off from the same subset as that of the TA-289 screens (Figure 2.12).

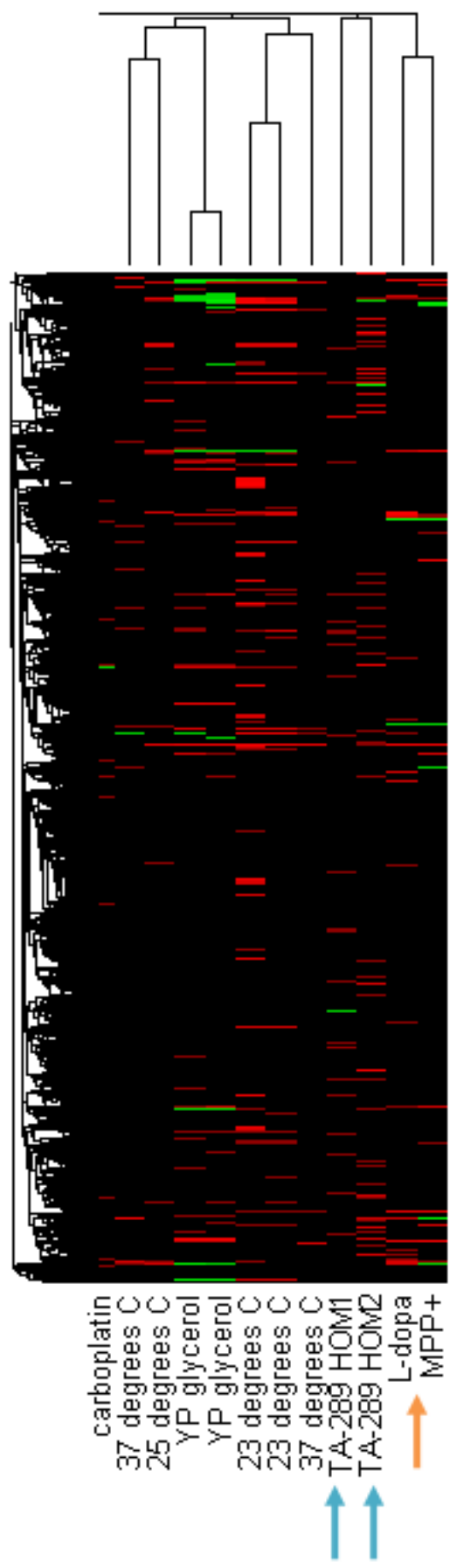

Figure 2.12. Close up clustergram of both TA-289 HOM screens (blue arrows). L-dopa and MPP+ are denoted with the orange arrow. See appendix 1.5 for the full clustergram. 
Chronic exposure to L-dopa, a drug clinically used for the treatment of Parkinson's disease, has been thought to enhance degeneration by causing oxidative stress through impairing mitochondrial respiratory capacity (Przedborski 1993). MPP+ (1methyl 4-phenylpyridinium) is a neurotoxin that acts on the electron transport chain in the mitochondria (Langston 1999). A total of 231 genes out of the combined 633 genes identified as hits (both sensitive and resistant) in both HOM screens (see supplementary file 1 for complete list and also the threshold set to determine sensitive and resistant genes) overlapped with genes identified as hits in the profile screens of L-Dopa and MPP+ (see appendix 1.6). Of these, 41 genes overlapping between the TA-289 HOM screens and the L-Dopa and MPP+ screens were identified as genes localized to the mitochondrion or the ER, and a further 16 genes were identified as those involved in DNA damage and repair and/or oxidative stress (see appendix 1.6). These, coupled with the list of sensitive genes obtained from the HET and HOM screens, suggest that TA-289 may act on the mitochondria in $S$. cerevisiae.

Protein localization data from a large-scale protein localization study (Huh, Falvo et al. 2003) of the gene hits from the second HOM screen were compared to that of the whole genome to identify enrichment or overrepresentation of any one cellular compartment. Gene hits identified in the HOM screen were primarily localized to the cytoplasm, however enrichment in the mitochondrion showed greatest significance $(\mathrm{p}<0.01)$, followed by the ER and Golgi $(\mathrm{p}<0.01)$ (Figure 2.13). 
Moreover, these biological processes, in particular the mitochondrion- and the ERrelated processes, are consistent with the previously described mechanisms of equisetin (Lundin, Deopujari et al. 1992; König, Kapus et al. 1993; Patham, Duffy et al. 2009). This is also further evidence that TA-289 and equisetin may share similar mechanisms-of-action.

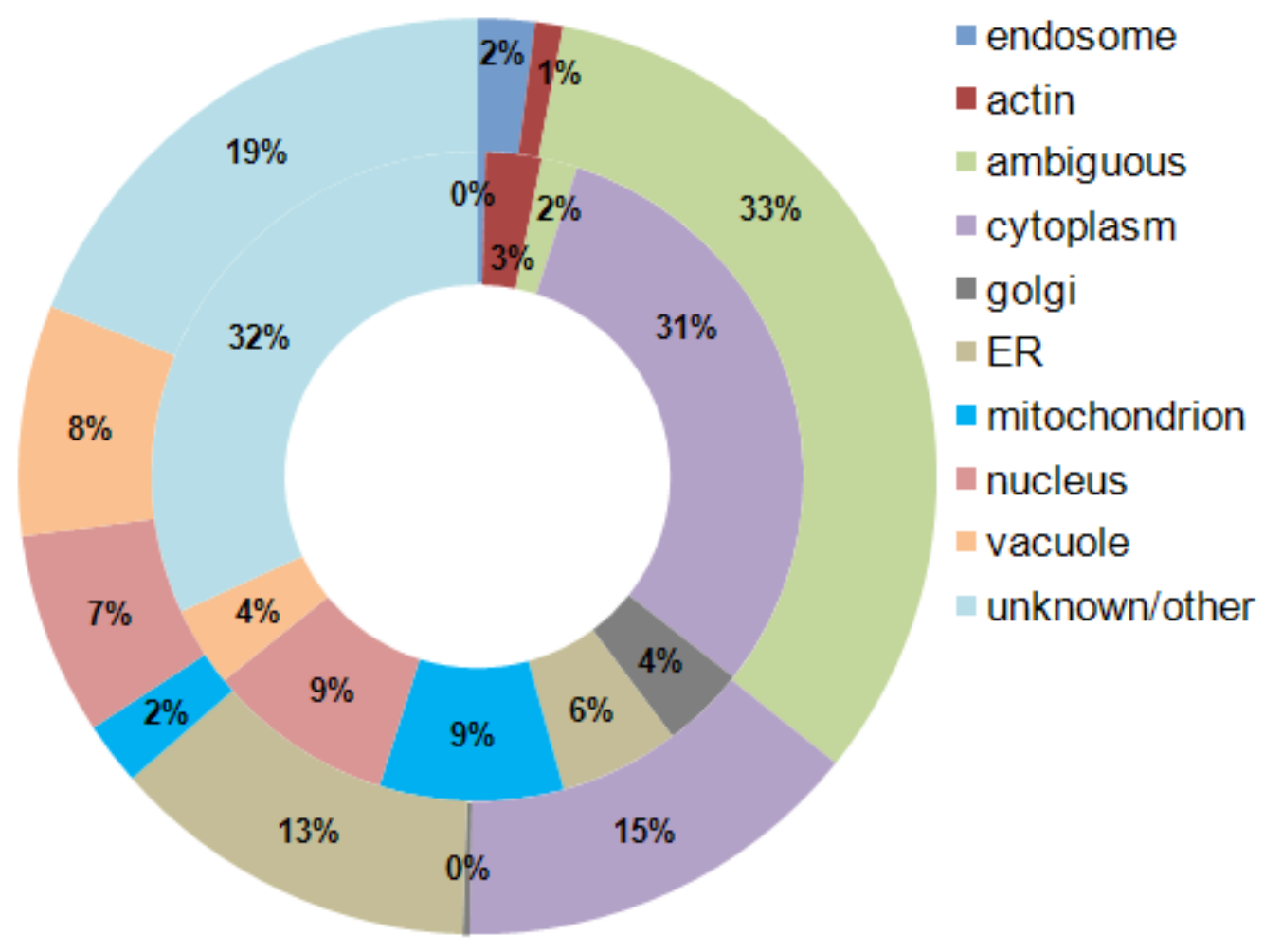

Figure 2.13. Gene localizations of HOM screen compared to whole genome. Gene hits from the second HOM screen (inner circle) were grouped according to their cellular component and compared to the whole genome localization (outer circle). 


\subsubsection{TA-289 and equisetin: Glucose vs. Glycerol as a carbon source}

In light of the previous studies which identified the mitochondria as a possible site of action for equisetin (König, Kapus et al. 1993), in combination with the evidence that TA-289 may be acting at the mitochondria from the chemical genomic profiling screens, the effects of TA-289 and equisetin on yeast cells were tested on fermentable (glucose) and non-fermentable (glycerol-ethanol) carbon sources. Firstly, liquid dose-responses were conducted using either glucose as a carbon source, or glycerol-ethanol. These determine dependency of mitochondrial respiration in the presence of TA-289 and equisetin. Prolonged treatment (three-day incubation for glucose) with TA-289 and equisetin showed that wt cells were able to adapt to growth in the presence of TA-289 and equisetin (Table 2.6). However, when glycerol-ethanol was used as the carbon source (six-day incubation for glycerol-ethanol), even after six days of incubation, cells treated with TA-289 and equisetin did not display the adapted resistance as that observed in glucose.

Table 2.6. MIC values of TA-289 and equisetin in wt and pdrA strains under different carbon sources. MIC values from the standard $18 \mathrm{~h}$ liquid dose-response assays are provided for comparison.

\begin{tabular}{|l|c|c|c|c|}
\hline \multirow{2}{*}{ Carbon source } & \multicolumn{2}{|c|}{ MIC $(\mu \mathrm{M})$ TA-289 } & \multicolumn{2}{c|}{ MIC $(\mu \mathrm{M})$ Equisetin } \\
\cline { 2 - 5 } & wt & pdr $\Delta$ & wt & dr \\
\hline $\begin{array}{l}\text { Glucose (standard } \\
18 \text { h assay) }\end{array}$ & 50 & 10 & 30 & 25 \\
\hline Glucose (day 3) & $>100$ & 10 & 50 & 25 \\
\hline Gly-EtOH (day 6) & 50 & 25 & 50 & 25 \\
\hline
\end{tabular}


Cell death assays used to determine the time at which it takes for TA-289 and equisetin to kill yeast cells were also compared between the two different carbon sources. When glucose is the carbon source, TA-289 does not kill BY4742 (wt) yeast, but kills $p d r \Delta$ cells at the highest concentration within the first 30 mins of treatment, and this potency increases within $2 \mathrm{~h}$ of treatment (Figure 2.14). The loss of potency observed when removing cells from a high concentration of TA-289 to a low concentration, i.e. from liquid and spotting onto solid, replicates the results observed in the CFU assay in chapter two (result 2.4), where BY4742 cells were able to recover.

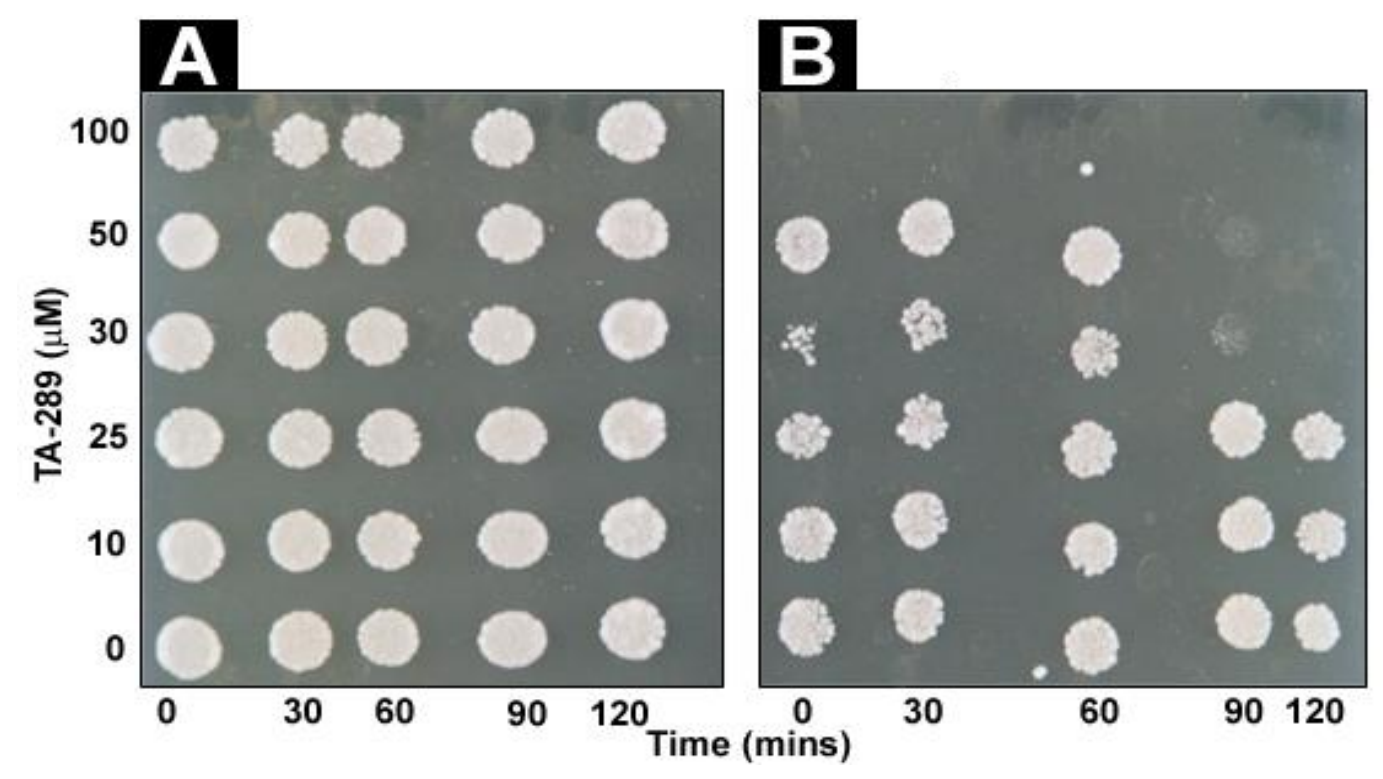

Figure 2.14. TA-289 kills pdrd cells within $2 \mathrm{~h}$ of treatment when glucose is used as a carbon source. wt (A) and pdr $(\mathrm{B})$ cells were treated with varying concentrations of TA-289 (y-axis), incubated at $30^{\circ} \mathrm{C}$ in SC, where cells were spotted onto agar at the time of treatment (0 mins), and then every 30 mins for $2 \mathrm{~h}$ ( $\mathrm{x}$-axis). 
In contrast, while cells did not gain resistance over time in glycerol-ethanol, they also do not die, as seen in pdr $\Delta$ cells where growth is observed even after $2 \mathrm{~h}$ of treatment, and where wt is inhibited only at the highest concentration at $2 \mathrm{~h}$ (Figure 2.15). Similar observations were noted for equisetin cell death assays conducted with $p d r \Delta$ cells. See appendix 1.7 for cell death assays with equisetin. An important observation to note is that TA-289 seems to kill wt cells after $2 \mathrm{~h}$ of treatment in glycerol-ethanol. This was not observed in glucose, where wt cells recovered strongly.

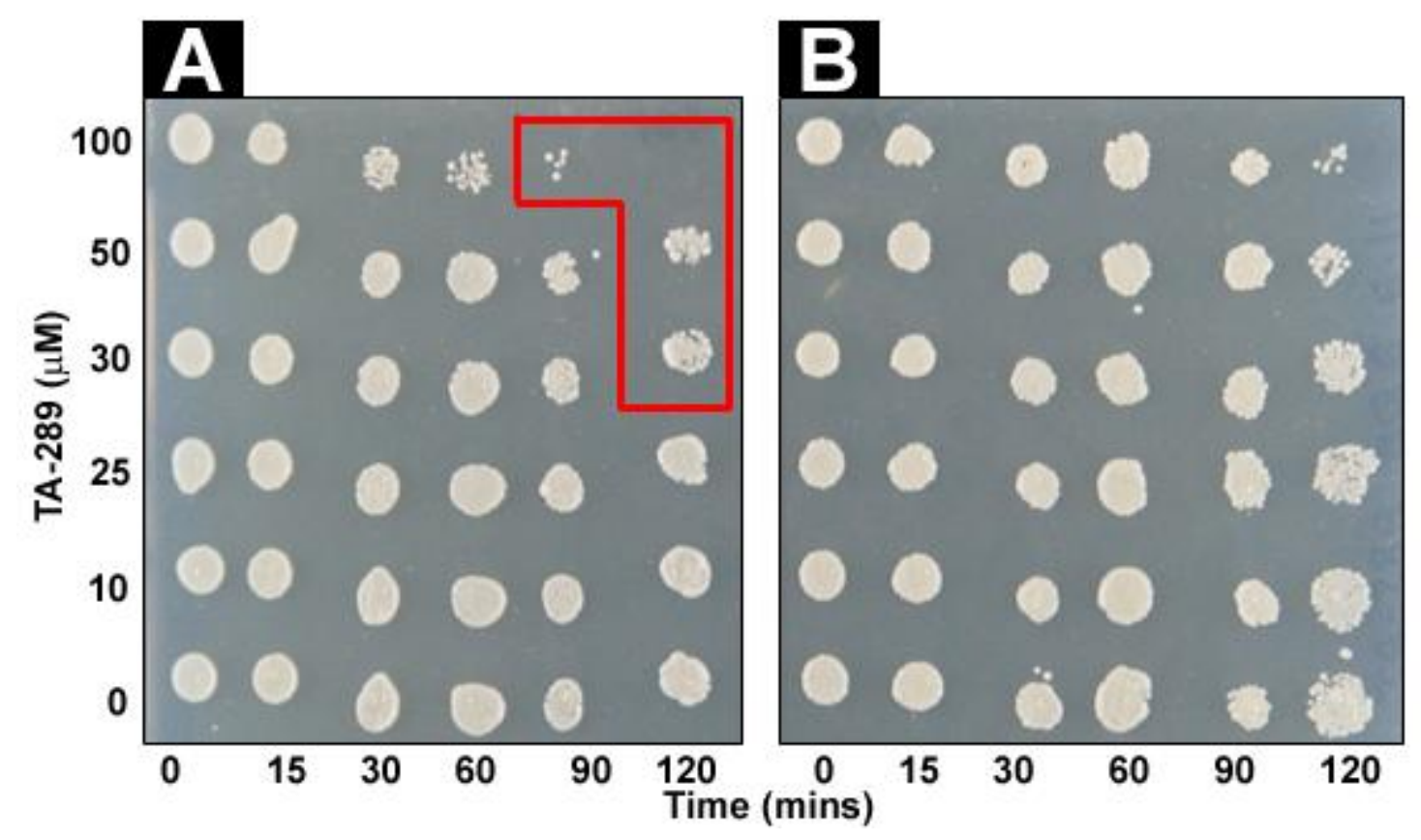

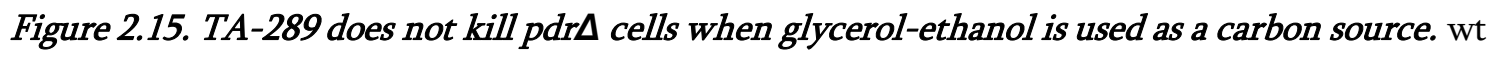
(A) and pdrA (B) cells were treated with varying concentrations of TA-289 (y-axis), incubated at $30{ }^{\circ} \mathrm{C}$ in $\mathrm{SC}+$ gly-EtOH, where cells were spotted onto agar at the time of treatment (0 mins), and then every 30 mins for $2 \mathrm{~h}$ (x-axis). Highlighted in the red box are wt cells that seem to be dead after 2 h treatment with TA-289 in glycerol-ethanol, which was not observed in glucose (Figure 2.14). 


\subsection{Discussion}

\subsubsection{Biologically active compounds from terrestrial fungi}

Fungal metabolites are diverse in biological activity, many of which possess favourable pharmacokinetic properties. A small library of natural products isolated from soil fungi was provided for drug mode-of-action study in yeast. Biological activities of the library of compounds ( 88 in total) were determined by treating two yeast strains and measuring the ability to grow in the presence of the compounds. For these initial screens, a compound was deemed biologically active where it caused greater than $70 \%$ growth inhibition compared to the solvent carrier control. Of the 88 compounds tested, 32 were identified as biologically active in BY4742 (wt) yeast, and an additional 10 compounds were identified as active only in the $p d r \Delta$ yeast strain at $100 \mu \mathrm{M}$ (Figure 2.1). Seven of the 32 compounds were novel and previously unreported, including TA-289, which was chosen for further study alongside equisetin, a known, structurally related compound (Burmeister, Bennett et al. 1974). Both compounds were isolated from terrestrial fungi of the genus Fusarium, and both displayed potent growth inhibitory effects on the two yeast strains tested. For the purpose of this study, TA-289 and equisetin were chosen due to their uncharacterized modes-of-action as well as their availability for study.

TA-289 is a novel bioactive metabolite, while the structurally related equisetin has been previously shown to exert its inhibitory activities through the mitochondrial 
substrate anion carriers in isolated rat mitochondria, and observed stimulatory effects on inorganic mitochondrial pyrophosphatase (Lundin, Deopujari et al. 1992; König, Kapus et al. 1993), in addition to inhibition of translocation of posttranslation proteins to the ER (Patham, Duffy et al. 2009).

TA-289 and equisetin display $\mathrm{pH}$-dependent growth inhibitory activities. This $\mathrm{pH}-$ dependency strongly suggests a requirement for the protonation of both compounds at low $\mathrm{pH}$ for more efficient penetrance through the cellular membrane before reaching their site of action (Cui, Hirata et al. 1996). It is speculated that once TA289 and equisetin cross permeate the cell membrane, the compounds become deprotonated as a result of higher intracellular pH levels (Valli, Sauer et al. 2005; Orij, Postmus et al. 2009) compared to that of the extracellular media, and thus become "trapped" in the cells unless they are actively removed. In yeast, the complex pleiotropic drug resistance network of efflux pumps allows efficient expulsion of xenobiotics from within the cell (Balzi, Chen et al. 1987). The generation of yeast strains deficient in the main transcription factors of these efflux pumps ( $P D R 1$ and its homologue, $P D R 3$ ) circumvents this problem. In this study, experiments were conducted using two yeast strains (BY4742 (wt) and pdrA - a strain deficient in $P D R 1$ and $P D R 3)$. Liquid and solid dose-responses revealed significant difference in the potency of both compounds between BY4742, and the $p d r \Delta$ strain. The potency of TA-289 and equisetin was significantly greater in the $p d r \Delta$ strain compared to 
BY4742. This efflux pump-sensitivity was not observed in the initial screening of the natural products library. Therefore, while growth inhibition assays performed at a single concentration serve to indicate biologically active molecules in yeast, they do not necessarily determine whether a given molecule is a substrate for yeast drugefflux pumps. This is especially so in the case where the molecule is potent in yeast, overcoming the $P D R$ network at higher concentrations and inhibiting growth of BY4742, as observed with TA-289 and equisetin. The differential sensitivity between BY4742 and pdrA cells to TA-289 and equisetin in the dose-response assays illustrates the effectiveness of making the cell more susceptible to xenobiotics through deletion of $P D R 1$ and $P D R 3$. Moreover, the increased potency observed in $\operatorname{pdr} \Delta$ cells strongly indicates both compounds as substrates for (at least one of) the yeast efflux pumps.

\subsubsection{Effects of TA-289 and equisetin on cell morphology and cell cycle}

Saccharomyces cerevisiae cells divide by a process known as budding, where a cell exiting $\mathrm{G}_{1}$ phase produces a small bud, which then continues to grow as the cell progresses through the stages of the cell cycle. As such, the size of the bud is indicative of the stage of the cell cycle in which any given cell is currently undergoing. Most importantly, cell cycle analyses and morphological studies provide leads to possible mechanisms-of-action of biologically active compounds of interest. For instance, actin inhibitor latrunctulin A causes a cell cycle block at $\mathrm{G}_{1}$ phase (small-budded phenotype) (Ayscough, Stryker et al. 1997), where perturbance of 
actin results in dysfunctional bud emergence as transport of cellular components and nutrients along actin cables are disrupted (Wen and Rubenstein 2009).

Cell cycle analysis by flow cytometry revealed cells treated with TA-289 and equisetin displayed a decrease in $\mathrm{G}_{1}$ phase and an increase in $\mathrm{S}_{-} \mathrm{G}_{2} / \mathrm{M}$ phase $4 \mathrm{~h}$ after treatment. Notably, it is difficult to distinguish between $S$ and $G_{2} / M$ phase in the yeast cell cycle, and the increase area observed as "S phase" could be for the most part, $\mathrm{G}_{2} / \mathrm{M}$. The increase of $\mathrm{G}_{2} / \mathrm{M}$ phase observed in yeast cells is often associated with tubulin-targeting compounds (Iwasaki 1992; Hamel 1996), where microtubule stabilization and/or destabilization lead to mitotic arrest. This results in a characteristic cell cycle block at $\mathrm{G}_{2} / \mathrm{M}$. A heterozygous diploid strain lacking TUB2 $(t u b 2 \Delta:: \operatorname{Kan} R)$, which encodes for the $\beta$-tubulin subunit of yeast microtubules, was tested for sensitivity to TA-289 and equisetin. In the case where microtubules are the target, a heterozygous diploid with only one wild-type copy of the target gene will be hypersensitive, as the reduced number of protein product is overwhelmed by the compound that binds to it at a lower concentration than in wild-type, a phenomenon known as drug-induced haploinsufficiency (Giaever, Shoemaker et al. 1999). The tub2 2 strain was no more sensitive to TA-289 or equisetin than the wt diploid, while it is hypersensitive to anti-mitotic agent, benomyl. This strongly suggests that neither TA-289 nor equisetin are tubulin-targeting compounds. 
Prolonged treatment (18 h) of TA-289 and equisetin cause both wt and pdr $\Delta$ cells to display a small-size phenotype, known as pyknosis, a characteristic associated with yeast apoptosis. Yeast have been shown to exhibit several key morphological features of apoptosis, and in pyknosis, reduction in cell size, chromatin condensation and subsequently DNA fragmentation indicates a dying cell (Chen, Dunigan et al. 2003). A positive correlation between reduction in cell size and the concentration of TA-289 and equisetin was tested. At the highest concentration of $100 \mu \mathrm{M}$, cells are 1.4 fold smaller than untreated controls $\left(\mathrm{P}=2.67 \times 10^{-16}\right)$. For cells treated at a lower concentration, $30 \mu \mathrm{M}$, size reduction is not as severe. These observations indicate that TA-289 and equisetin cause a dose-dependent apoptotic-like phenotype in yeast.

A decrease in potency of both TA-289 and equisetin was observed in BY4743 diploid wt yeast cells (used as a control strain when testing the tub2 2 diploid mutant) compared to that of haploid BY4742 wt yeast cells (used in normal dose-response assays), with an approximated MIC of $50 \mu \mathrm{M}$ for TA-289 in haploid BY4742 cells, compared to $>100 \mu \mathrm{M}$ in diploid BY4743 cells, and $30 \mu \mathrm{M}$ for equisetin in haploid BY4742 cells, compared to $50 \mu \mathrm{M}$ in diploids. Arguably, a haploid cell would contain half the number of target protein that would otherwise be present in a diploid cell, thus requiring less drug-target interactions to cause growth inhibitory effects. This situation leads to an apparent higher potency observed in haploid cells. Moreover, 
the decreased potency can also be attributed to the presence of two gene copies of the transcription factors (PDR1 and PDR3) and drug efflux pumps that drive most of the yeast pleiotropic drug resistance genes in the diploid BY4743 cell, leading to a more efficient efflux pump system compared to the haploid BY4742 cell. In saying that however, diploid yeast cells tend to be twice the volume compared to haploid yeast cells (Amberg, Burke et al. 2005), and therefore the effective $P D R$ protein concentration may be similar between a diploid and a haploid ell.

Cell growth inhibition is indicative of a biologically active molecule. To determine whether these inhibitory effects are irreversible, cells treated with TA-289 and equisetin were washed and allowed to recover on rich media. Interestingly, BY4742 cells treated with TA-289 and equisetin were able to recover after washing, while pdr $\Delta$ cells did not recover. This was also observed in the time-course cell cycle analysis of treated cells by flow cytometry, where BY4742 cells recovered within 1.5 $h$ after washing off TA-289 and equisetin, as indicated by a return of the $\mathrm{G}_{1}$ peak in TA-289-treated BY4742 cells, and a slower return in equisetin-treated BY4742 cells. The slower recovery of BY4742 cells treated with equisetin may be attributed to the fact that equisetin is more potent to yeast than TA-289, and this increased potency is postulated as a result of the compounds ability to either more effectively enter the cell and/or bind to its target(s) with higher affinity. As TA-289 and equisetin are substrates of the yeast efflux pumps, it is possible that BY4742 wt cells were able to 
remove TA-289 and equisetin from within the cell at a sufficient rate before the compounds were able to elicit their irreversible inhibitory effects. This allowed BY4742 cells to recover from treatment with TA-289 and equisetin, in contrast to the more sensitive $p d r \Delta$ cells. As such, it can be suggested that TA-289 and equisetin cause either cell death or irreversible growth inhibition in yeast, however the effect is only reflected in $p d r \Delta$ cells lacking in an efficient efflux pump network. However, cell death assays observed in result 2.5.6 indicate the ability of TA-289 and equisetin to kill wt yeast cells in respiratory conditions (in glycerol-ethanol). This will be further discussed in section 2.5.4 of this discussion.

\subsubsection{Chemical genomic profiling of TA-289}

The advent of yeast genomics technologies have allowed for genome-wide study of the mechanisms of a biologically active compound. Chemical genomic profiling in particular utilises an inhibitory compound as a chemical probe, hand-in-hand with the yeast genome deletion sets to reveal affected biological processes. These in turn provide leads into further understanding the mechanism of a compound. In this study, two yeast genome deletion sets were screened against TA-289; the homozygous diploid deletion set (HOM), comprising of the complete homozygous deletion of nonessential genes from the $S$. cerevisiae genome, and the heterozygous diploid deletion set (HET), which are heterozygous deletions that thus include the essential genes of the yeast genome. While haplo-insufficiency profiling (as performed in the HET screen) is based on identifying the most sensitive hit as the 
drug target, often this may not be so straight forward. Nevertheless, the combination of both screens allows for greater understanding of the mechanisms of TA-289.

To this end, a number of gene deletions identified as sensitizing to TA-289 from the HOM and HET screens (top 40 and top 15, respectively) were associated with oxidative stress and DNA damage (RTT107, RPN4, STB5, CNB1, DPB3, and YBP1), mitochondria (ARC18, ILM1, ILM2, MSW1, CIN8, NPL4, YJR100C, MTG1, YLR201C (COQ9), YDR438W, MIP1, SNL1, AUT7, APJ1, and TOM22), translation and/or transcription, and regulation of transcription from RNA polymerase II promoter (RPS27B, INO2, PHO2, PHO23, SNF4, FZF1, GAL11, ARR1, LSM4, and MED4), endocytosis, vacuolar transport, and ER (VPS35, YKR007W, RAV1, GTR1, MON2, YIP4, GDA1, PRB1, and SIN3). Hierarchical cluster analyses showed both HOM screens displayed similarities (wherein they branched from the same subset in the cluster analyses) with L-Dopa and $\mathrm{MPP}+$, a therapeutic drug and a neurotoxin thought to exert at least some of their biological effects by acting at the mitochondria and causing oxidative stress (Przedborski 1993; Langston 1999). Additionally, when localizations of gene hits from one of the HOM screens were compared to whole genome localization, a significant overrepresentation was found in genes localized to actin, the nucleus, Golgi, ER, and most significantly at the mitochondrion. 
One of the top ten sensitive heterozygous deletion strains from the profiling screens was aut $7 \Delta$, an autophagy-related gene involved in autophagic removal of mitochondria induced by apoptotic factors. The aut7 null mutant displays reduced mitochondrial potential, reduced electron transport chain activity, cell cycle delay, and elevated ROS (Zhang, Qi et al. 2007). Haplo-insufficiency profiling has previously been shown to identify the mode-of-action of a biologically active compound (Baetz, McHardy et al. 2004; Lum, Armour et al. 2004), however, success can be variable, and in many cases the most sensitive hits from HET screens are often those involved in the consequential cellular processing following blocking of the mechanism of the drug rather than the target itself (Sturgeon, Kemmer et al. 2006). As such, it is possible that an aut7 null mutant strain displays significant sensitivity to TA-289 as it is unable to degrade dysfunctional mitochondria and other damaged proteins induced by TA-289.

Other interesting gene deletions that sensitized cells to TA-289 included tom22 4 , a component of translocase complex responsible for mitochondrial protein import, of which the deletion mutant displays cell cycle progression delay (Sopko, Huang et al. 2006) and abnormal mitochondrial morphology (Altmann and Westermann 2005). Likewise, while it was not represented as a top hypersensitive hit in the HET screen, the ppa2 $\triangle$ heterozygote still displayed relative sensitivity. $P P A 2$ is a mitochondrial inorganic pyrophosphatase required for mitochondrial function, the absence of 
which results in abnormal mitochondrial morphology (Dimmer, Fritz et al. 2002); equisetin has been previously showed to stimulate $P P A 2$ activity (Lundin, Deopujari et al. 1992). Mitochondrial porin POR1, a voltage-dependent anion channel is required for mitochondrial membrane osmotic stability and permeability. Por1p has been previously identified as a putative target for SFK1, a mitochondrial respiratory chain inhibitor. The por $1 \Delta$ heterozygote was identified as sensitive to TA-289 in one of the HOM screens and in the HET screen.

DNA damage repair and oxidative stress response genes identified in these screens included those that were previously identified as genes that sensitize most to DNAdamaging and oxidative stress inducing agents such as $\mathrm{H}_{2} \mathrm{O}_{2} ; R A D 1, R A D 5, R A D$, RAD17, $R A D 18, R A D 55, R A D 57, R A D 59, M M S 4$ (response to damage by alkylating agents) (Xiao, Chow et al. 1998), MEC3 (part of the rad7p complex) (Cardone, Revers et al. 2006), RPL20A (a ribosomal protein) (Planta and Mager 1998), and YIM1 (mitochondrial inner membrane protease) (Birrell, Brown et al. 2002). Most interestingly, and perhaps highlighting the potential of the mitochondrion being the site of action of TA-289 (and possibly equisetin, as previously described by König et. al.), is the large number of genes associated with the electron transport chain (ETC). This included genes involved in complexes I through $\mathrm{V}(\mathrm{NDE}, S D H, C O Q, C O X$, and $Q C R)$, and genes associated with ADP/ATP carriers (PET). In concert, many of the genes identified in the chemical genomic profiling of TA-289 suggest two factors, 
firstly, that the ER and especially the mitochondrial processes (due to the significant enrichment of genes localized to the mitochondrion) identified in these screens were consistent with previous reports of inhibition of post-translational protein import to the ER (Patham, Duffy et al. 2009), and also the substrate anion carriers of the mitochondrial respiratory chain by equisetin (König, Kapus et al. 1993), and secondly, is further evidence that TA-289 and equisetin may share similar a similar mechanism-of-action.

\subsubsection{Glucose vs. Glycerol as a carbon source}

Yeast are able to ferment and respire. In the presence of glucose, yeast preferentially undergo fermentation, whereas in glycerol-ethanol, they actively respire. Yeast cells displayed differences in growth inhibition when treated with TA-289 or equisetin depending on the carbon source used; either fermentative (glucose) or respiratory (glycerol-ethanol). The ability for cells to adapt to treatment of the two compounds appeared to be dependent on carbon source, in that TA-289 and equisetin kill yeast in glucose, but yeast cell death occurs slower in glycerol. Often, compounds targeting the mitochondria, in particular the mitochondrial respiratory chain, display greater potency (or are only biologically active) in respiring conditions (Li, Mo et al. 2005). In the case of TA-289 and equisetin, both compounds were active in fermentative and respiratory conditions. Therefore, if the target(s) is involved in the mitochondrial respiratory chain (as indicated by gene hits associated with the mitochondria, the significant localization of gene hits at the mitochondrion, and the 
mitochondrial respiratory chain i.e. ETC), then it is presumed that within a fermenting cell, there is some basal level of respiration, as TA-289 and equisetin are still growth inhibitory towards yeast.

Interestingly, when glycerol-ethanol is used as a carbon source, cells are more resistant to the death caused by TA-289 and equisetin, as observed in the cell death assays where even at $100 \mu \mathrm{M}, p d r \Delta$ yeast cells were only severely inhibited after two hours, compared to the complete death of cells within an hour of treatment when glucose is used. In glucose, yeast respire very little, and assuming that due to the low basal level of respiration, the target (presumably a protein) is also expressed at low levels, requiring less amount of drug (in this case, TA-289 and equisetin) to bind to the target in order to cause an effect. This will then result in a seemingly more potent effect of both TA-289 and equisetin under fermentative conditions.

However, when comparing the cell death results to the liquid growth dose-responses over an $18 \mathrm{~h}$ time period, cells are no less resistant to TA-289 and equisetin in glycerol-ethanol compared to glucose in liquid. Moreover, over prolonged exposure of wt cells to TA-289 and equisetin in liquid, cells are able to gain resistance in glucose, but not glycerol-ethanol. Therefore an alternative and a more probable scenario, is that when the cell is fermenting, the target of TA-289 and equisetin, though expressed, is non-essential, and overtime the cell is able to adapt by 
upregulating some sort of multi-drug resistance mechanism. This suggests that when glycerol-ethanol is the carbon source and cells are actively respiring, the target thus becomes essential, possibly because it is required for respiration, and so TA-289 and equisetin seem to inhibit the growth of cells in liquid over a longer period of time (up to six days).

Most importantly, while TA-289 kills $p d r \Delta$ cells within the first hour of treatment in glucose (as observed in the cell death assay figure 2.14), wt cells recovered. However, some wt cell death was observed in glycerol-ethanol (figure 2.15). This strongly indicates that the respiratory state of yeast is critical for the ability of TA289 to kill, and is further evidence that the mitochondria is heavily involved in the way which TA-289 causes yeast cell death. Interestingly, while $p d r \Delta$ cells were extremely susceptible to TA-289 in glucose, they showed resistance in glycerolethanol. This is unexpected because TA-289 has been shown to be a substrate for the yeast efflux pumps, and thus should be more potent to $p d r \Delta$ cells than it is to wt cells. It is speculated that this resistance may be due to pdr $\Delta$ cells holding higher antioxidant capacity than wt cells, where it is observed that $p d r \Delta$ cells were more resistant to TA-289 in glycerol-ethanol (figure 2.15). It is therefore suggested that the antioxidant capacity of a cell plays a more key role in a cell's ability to grow in the presence of TA-289 than that of the efflux pump capacity. Similar results were observed for cell death assays conducted with equisetin in different carbon sources. 
Possibly, greater yeast cell death may be observed over a longer period of time, as cell death assays were only recorded for $2 \mathrm{~h}$.

$S$. cerevisiae display similar cell death characteristics to that of mammalian apoptosis, including caspase-initiated apoptosis, and apoptotic phenotypes such as pyknosis (as observed in morphological assessment of yeast cells treated with TA289 in result 2.5.3); a reduction in cell size, chromatin condensation and DNA fragmentation (Madeo, Carmona-Gutierrez et al. 2009). The apoptotic pathway in yeast is similar to the intrinsic apoptotic pathway in mammalian cells, and is regulated by several key mechanisms including mitochondrial release of apoptosisinducing factor AIF1 (Wissing, Ludovico et al. 2004), yeast suicide protein YSP1 (Pozniakovsky, Knorre et al. 2005), cytochrome $c$ (Skulachev 1996), and activation of yeast metacaspase MCA1 (Madeo, Herker et al. 2002). The mitochondrion has been shown to be an important regulator of programmed cell death (PCD) (Skulachev 1996; Madeo, Herker et al. 2004; Eisenberg, Büttner et al. 2007). The differential potency and time it takes for yeast to die in the presence of TA-289 and equisetin in different metabolic states (i.e. fermentative or respiratory) is indication that PCD upon treatment with TA-289 and equisetin may be due to impairment of mitochondrial function as a result of the binding of both compounds to a target(s) that is intimately associated with the mitochondrion. 
The chemical genomic profiling screens of TA-289 allowed for the identification for potential mechanisms of the compound, with particular interest centred around the mitochondrial-related processes (König, Kapus et al. 1993). The previously described mechanisms of equisetin and the genes identified in the microarray screens for TA289 indicate that both compounds may share similar modes-of-action. The ability of TA-289 and equisetin to kill yeast appears to be dependent on carbon source, where the capacity for cells to respire may play a role in sensitizing to or giving resistance to TA-289 and equisetin, and that this strongly indicates a potential site-of-action at the mitochondria. 


\section{RESISTANT MUTANT GENERATION}

\subsection{Introduction: Resistant mutant generation}

Drug resistant mutant generation and characterization as an approach to determine the modes-of-action of various biologically active compounds has been successfully applied in various model organisms. In $S$. cerevisiae, its ease of manipulation and genetic tractability makes it an ideal eukaryotic organism to generate drug resistant mutants and to determine the gene(s) conferring resistance. In this way, the targets of many biologically active small molecules have been successfully identified in $S$. cerevisiae, including nystatin (Ahmed and Woods 1967), oligomycin (Wakabaya.K and Gunge 1970), reveromycin (Miyamoto, Machida et al. 2002), and rapamycin (Singh, Sun et al. 1979).

However, while the generation of mutants resistant to inhibitory compounds is fairly straightforward, the same cannot be said for identifying the gene in which the mutation has occurred, and determining the relationship of the mutation to the target of the compound of interest. Resistance to a growth inhibitory small molecule can occur in many ways, often depending on the consequence of the target-protein function and of the interaction of the target with the compound, as well as the nature of the mutation itself. A mutation affecting resistance can arise from, 1) the up-regulation of an adaptive response such as the multidrug resistance pumps to more efficiently export xenobiotics (Balzi and Goffeau 1995), 2) alteration of a gene 
that is required to activate and/or inactivate a compound, 3) alteration of a gene that affects the target(s) of a compound, or less usually but more simply 4) alteration of a gene that is the target of the compound. In the case of cycloheximide, which elicits its growth inhibitory effects by inhibiting protein synthesis through targeting the ribosome, a number of mutants found to confer resistance to cycloheximide ranged from recessive, to semi-dominant, and dominant mutations (Middlekauff, Hino et al. 1957; Wilkie and Lee 1965; Sutton, Ares et al. 1978; Stocklein and Piepersberg 1980; Fried and Warner 1982; Kaaufer, Fried et al. 1983).

For compounds that target large complexes with multiple subunits such as cycloheximide, it is not surprising that mutations in any one of the subunits within the ribosomal complex has been shown to provide resistance with such a spectrum of characteristics (Sutton, Ares et al. 1978; Stocklein and Piepersberg 1980; Fried and Warner 1982; Kaaufer, Fried et al. 1983). Likewise, resistance to small molecules that cause growth inhibition and/or death by the generation of reactive oxygen species, such as hydrogen peroxide, is a common example of an adaptive mechanism, wherein cells up-regulate, through a variety of means, intracellular anti-oxidant capacity to counteract the generation of reactive oxygen species (Dawes 2004; Thorpe, Fong et al. 2004). 
Underpinning any of these possibilities in explaining and characterizing a drug resistant mutant is the necessity of first determining the mutant lesion. An approach commonly used to determine a site of mutation is to create a plasmid-borne genomic library of a resistant mutant and transform the library into the parental strain from which the resistant mutant was derived. The fragment that gives rise to resistance is selected by growing the transformed parental strain on drug-containing media, after which the fragment is sequenced and the point of mutation identified (Fried and Warner 1981). This was applied to identify the cellular targets of reveromycin A, where strains containing a high-copy plasmid overexpressing the ILS1 (encoding tRNA synthetase, IleRS) gene conferred resistance to reveromycin A. Inhibition of tRNA synthetase in vivo was also observed, further indicating IleRS as the target of reveromycin A (Miyamoto, Machida et al. 2002).

Another approach takes advantage of a microarray-based growth assay combined with a commercially available plasmid library of molecular barcoded ORFs, to transform a drug resistant mutant. This approach allows identification of recessive mutations through mapping by complementation, as the transformant that receives the wild-type copy of the drug resistance gene will be unable to grow in the presence of the drug. As each ORF is uniquely barcoded, identification on an oligonucleotide microarray is possible, and the mutant gene may be subsequently cloned and sequenced (Ho, Magtanong et al. 2009). 
To date, while many approaches are used to determine drug resistance-conferring mutations, the challenge remains in identifying the true target of a small molecule of interest - lest we forget that some biologically active compounds have multiple targets, a classic example being salicylate and aspirin (Kopp and Ghosh 1994; Vane 1994). As mentioned earlier, there are many ways a cell can become resistant to a drug. Moreover, there is no correlation between the dominant-recessive nature of a resistant mutant, and the nature of the gene as a drug target. Nevertheless, the characterization of the nature of a drug resistant allele undoubtedly provides valuable information, both in mapping the mutation itself, and towards understanding the mechanism of the drug.

\subsection{Synthetic genetic array mapping}

SGA (Synthetic Genetic Array) analysis (Tong, Evangelista et al. 2001), is an assay that explores phenotypic enhancement between gene pairs. SGA analysis, in short, involves crossing a query strain comprising the deletion mutant of interest carrying a nourseothricin (NAT) antibiotic resistance marker to the genome-wide homozygous deletion set carrying the kanamycin (G418) resistance marker in an ordered array, en masse. Double mutants are thus able to grow in the presence of both antibiotics (Boone and Tong, 2005). These basic attributes of SGA have also proven to be a powerful tool for a high-throughput mapping of mutations (Jorgensen, Nelson et al. 2002) in a procedure called SGA mapping (SGAM). This methodology relies on a key fact, where if the query allele, in this case an unknown 
mutation, and the deletion mutant array (DMA) allele are the same, only one antibiotic marker will be present, causing cells to die when selected on both antibiotics. This appears as a visible no-growth hole in SGA culture plates.

Linkage disequilibrium is a phenomenon whereby an allele occupying a locus at any given site on a chromosome does not segregate independently from another allele occupying a second nearby locus because of low meiotic recombination frequency of the adjacent or nearly adjacent alleles. In the case just described, the characteristic no-growth/growth of query/array genes occupying the same locus will spread to approximately half-dozen or so genes on either side of that locus, forming a clear visual display or linkage group of no-growth. This effectively maps the location of the query mutation on the SGA array. A novel application of the SGAM method in our laboratory has been to map drug resistant mutations, such that essentially, the drug/drug resistant mutant pair replaces the query antibiotic/antibiotic marker pair described above; a procedure called C-SGAM (chemical-synthetic genetic array mapping) (Ploi Yibmantasiri, manuscript in preparation).

The C-SGAM method of mutation mapping utilizes the generation of a mutant yeast strain resistant to a bioactive molecule of interest that is crossed with the yeast deletion set following the SGA methodology. As described, taking advantage of drug 
resistance as a selectable marker, the final pinning procedure in the SGA therefore involves double mutant selection on drug-containing (to select for the presence of the resistant mutation) and G418 (geneticin)-containing media (to ensure the presence of the original $\mathrm{xxx} \Delta$ genes from the DMA) (Figure 3.1). In the event where the mutation allele occupies the same locus as a particular gene in the deletion array $(\operatorname{xxx} \Delta)$, recombination cannot occur as both resistant alleles cannot occupy the same locus, resulting in inviable progeny when grown in the presence of both G418 and the drug of interest. A symmetrical linkage pattern is therefore often observed where growth defect is inversely proportional to the probability of meiotic recombination between neighbouring genes of the gene target (Figure 3.2) i.e. linkage disequilibrium. This pattern identifies the position of the locus of resistance, thus narrowing the area of the genome which may contain the drug target (or at the very least, the resistance-conferring gene mutation) of the compound of interest. 


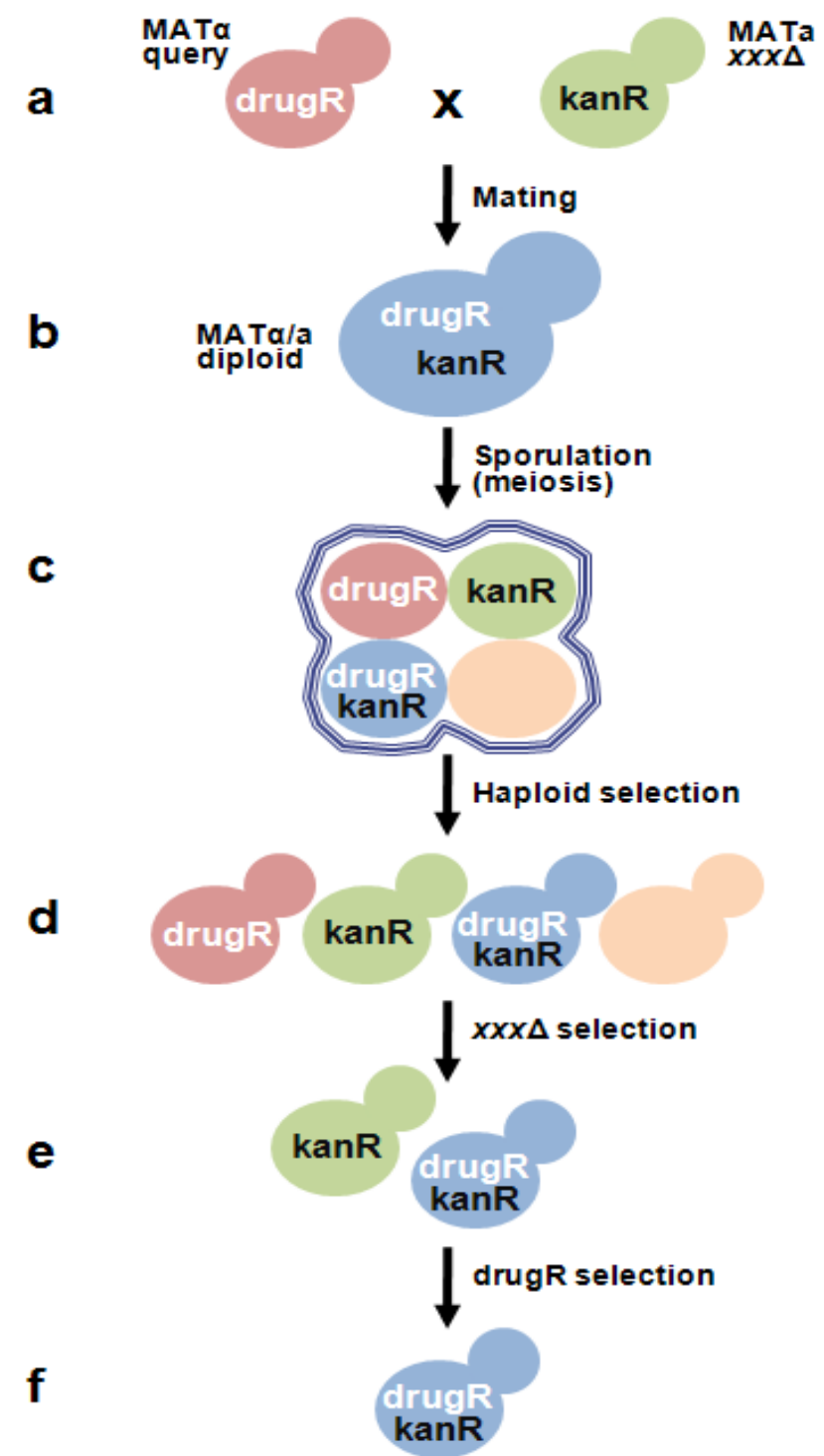

Figure 3.1. A simplified flow diagram of the C-SGAM methodology. (a) A MAT $\alpha$ strain carrying the drug-resistant mutation of interest (drugR - aptly named, the "query strain"), is crossed with the haploid yeast deletion set, all of which are of mating type MATa, and in place of every gene deletion $(\mathrm{xxx} \Delta)$ is a dominant kanamycin-resistance $(\mathrm{kanR})$ selectable marker to produce a diploid strain (b). The resulting heterozygous diploids are induced to sporulate (c), after which only the MATa meiotic progeny that express certain SGA reporters are selected (d). (e) Selecting for kanR ensures original deletion array mutants are present $(\operatorname{xxx} \Delta)$, and the final double mutant selection step in drug- and G418-containing media (f), selects for double mutants containing the mutation of interest (drugR) and the deletion mutant from the array (kanR). 

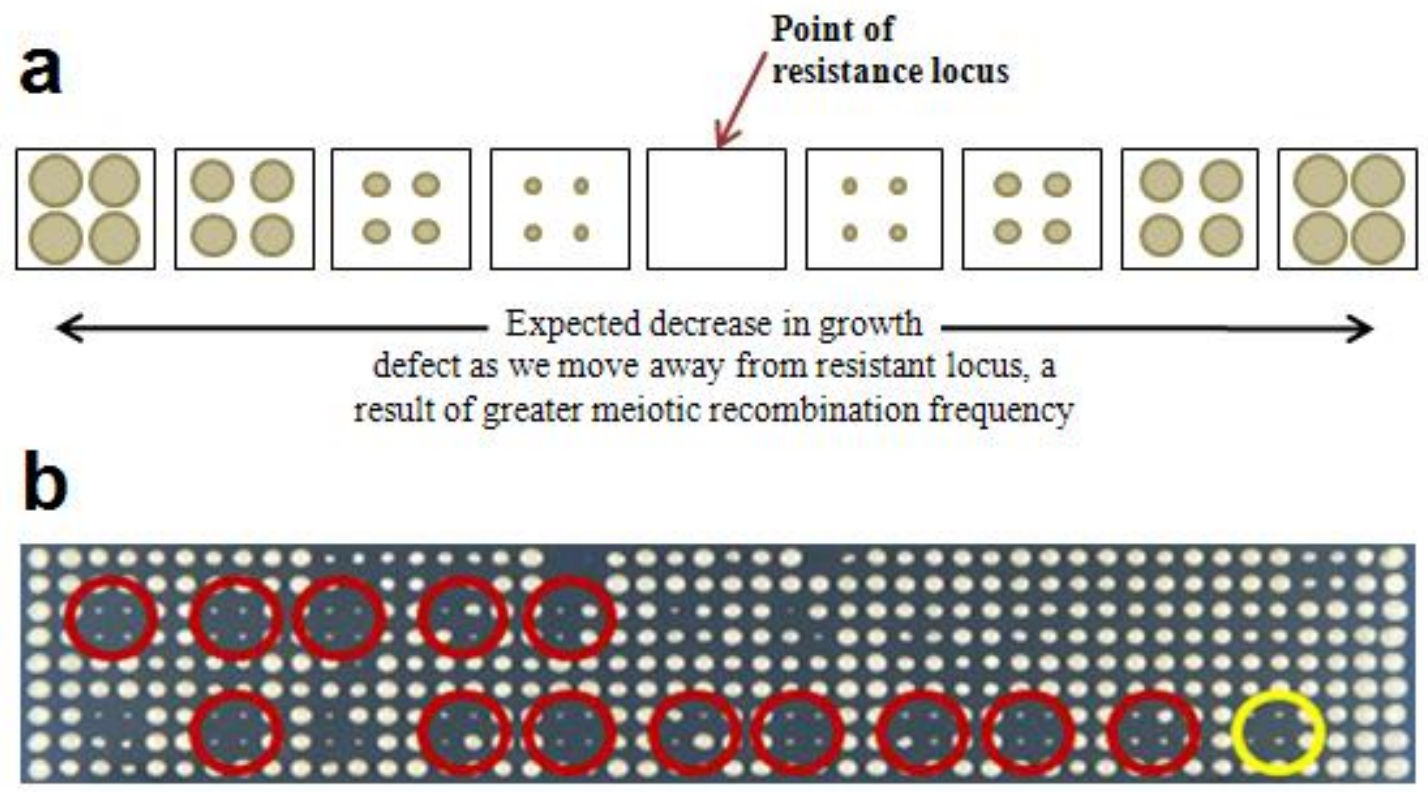

Figure 3.2. Visual representation of linkage disequilibrium. The chromosomal location of a resistant allele can be identified by observing the growth patterns of yeast colonies on an arrayed plate containing double selection media (a). An example of a linkage pattern is shown in (b), where the target gene $A D H 2$ is highlighted in yellow and linkage genes are in red. Figure (b) obtained from Bede P. Busby 2007 final project report.

A major flaw in the SGA methodology - this is especially so when utilizing SGA methods to map drug resistant mutations i.e. the C-SGAM - lies in the quantity of biologically active compounds required to perform the final selection on a large volume of solid media. In the case where a compound of interest is limited in quantity, as it is often with purified natural products, and it is so for the novel compound, TA-289, the application of a microarray-based growth assay may be used as a mapping tool, i.e. measuring the amount of barcode present for each gene deletion from a genome-wide pool of "array mutants" grown in a small volume in the presence of the compound. Fluorescence intensities are then used to identify linkage disequilibrium patterns on a microarray chip, paralleling the colony growth 
used to identify patterns on agar plates in a standard SGAM method. This novel method of mapping by microarray will allow the assessment of gene fitness through pooled competitive growth, and as such requires much less drug than what is needed for SGA. 


\subsection{Aims and objectives}

The aim of this chapter was to explore the mechanisms-of-action of TA-289 and equisetin through the generation of mutants resistant to TA-289 and equisetin, and then by mapping the resistance-conferring gene(s) by combining two established yeast methodologies:

- Resistance mapping

- Microarray growth assay

Part of this study also involved a proof-of-principle for the novel method of combining the C-SGAM methodology with mapping by microarray at the final SGA selection step by using a characterized mutant and the compound to which it is resistant as a pilot study. 


\subsection{Materials and methods}

\subsubsection{Yeast strains}

All $S$. cerevisiae strains used in this study are as follows:

\begin{tabular}{|c|c|}
\hline Strain & Genotype \\
\hline YCG191 (CYH') & $\begin{array}{l}\text { MAT } \alpha \text { can1 }:: S T E 2 p \_L E U 2 ; 1 y p 1 \Delta ; \text { his3 } 11 \text { leu2 } 20 \\
\text { ura3 } \Delta 0 \text { met } 15 \Delta 0\end{array}$ \\
\hline YCG326 (pdr $\Delta)$ & 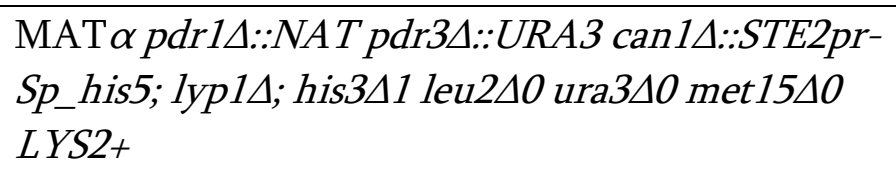 \\
\hline YCG387 & 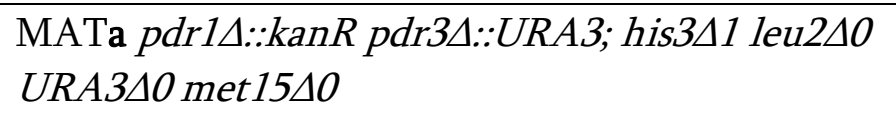 \\
\hline $\operatorname{pdr} 1 \Delta(\operatorname{pdr} 1 \Delta-\mathrm{p})$ & Derived from $B Y 4741$; pdr1A::kanR \\
\hline
\end{tabular}

\subsubsection{Growth media}

All yeast cultures were performed in one of the following media as prescribed previously, supplemented with either G418 (200 $\mu \mathrm{g} / \mathrm{mL}$ final concentration), NAT (100 $\mu \mathrm{g} / \mathrm{mL}$ final concentration), or Can $(50 \mu \mathrm{g} / \mathrm{mL})$ and Thia $(50 \mu \mathrm{g} / \mathrm{mL})$ (SigmaAldrich), unless otherwise stated.

\subsubsection{Compounds and chemicals}

Purchased from Sigma-Aldrich Life Science and Biochemicals: Ethane methyl sulfonate (EMS) stored at room temperature, sodium thiosulfate $\left(\mathrm{Na}_{2} \mathrm{~S}_{2} \mathrm{O}_{3}\right)$ at $50 \%$ and $5 \%$ working concentrations prepared in $\mathrm{ddH}_{2} \mathrm{O}$ and stored at room temperature, dibasic and monobasic sodium phosphate $\left(\mathrm{Na}_{2} \mathrm{HPO}_{4}, \mathrm{NaH}_{2} \mathrm{PO}_{4}\right.$, respectively) at 
$0.2 \mathrm{M}$ working stocks prepared in $\mathrm{ddH}_{2} \mathrm{O}$ and stored at room temperature, cycloheximide, $10 \mathrm{mg} / \mathrm{mL}$ working stock prepared in DMSO and stored at $-20{ }^{\circ} \mathrm{C}$, dithiothreitol (DTT), $1 \mathrm{M}$ working stock prepared in $\mathrm{ddH}_{2} \mathrm{O}$ and stored at $-20{ }^{\circ} \mathrm{C}$, hydrogen peroxide $\left(\mathrm{H}_{2} \mathrm{O}_{2}\right)$, 3\% working stock prepared in $\mathrm{ddH}_{2} \mathrm{O}$ and stored in the dark at $4{ }^{\circ} \mathrm{C}$.

\subsubsection{Resistant mutant generation by ethane methyl sulfonate mutagenesis}

Liquid cultures of YCG326 ( $p d r \Delta$ ) were grown overnight in $2 \mathrm{~mL}$ SC media at $30{ }^{\circ} \mathrm{C}$ on a rotating drum. Mutagenesis was adapted from a standard protocol (Amberg 2005). Cells were collected by centrifugation, washed once with $1 \mathrm{~mL} \mathrm{ddH}_{2} \mathrm{O}$, and pellets resuspended in $1 \mathrm{~mL} 0.1 \mathrm{M}$ sodium phosphate buffer ( $\mathrm{pH}$ 7.0). To one culture, $30 \mu \mathrm{L}$ of ethane methyl sulfonate (EMS) was added, giving a final concentration of 3\% EMS, while the other culture remained as an unmutagenized control.

Cells were lightly vortexed and incubated at $30{ }^{\circ} \mathrm{C}$ for $1 \mathrm{~h}$ on a rotating wheel, after which the cells were collected by centrifugation, supernatant discarded into a beaker containing $50 \%$ sodium thiosulfate, and pellets gently washed twice with

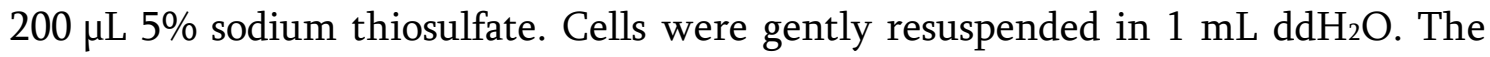
cultures were transferred to fresh, sterilized $15 \mathrm{~mL}$ falcon tubes, further centrifuged, and cell pellets resuspended in $2 \mathrm{~mL}$ YPD, after which the tubes were incubated at $30{ }^{\circ} \mathrm{C}$ for $2 \mathrm{~h}$ on a rotating wheel. 
A $20 \mu \mathrm{L}$ aliquot of the mutagenized and unmutagenized cultures were inoculated on to petri plates containing $20 \mathrm{~mL}$ SC, or SC with either $60 \mu \mathrm{M}$ TA-289, or $60 \mu \mathrm{M}$ equisetin, giving a final concentration of 20000 cells per plate. The plates were incubated at $30{ }^{\circ} \mathrm{C}$ for three to four days, and a selection of mutants, both spontaneous and EMS-generated, and of various sizes, were picked off and restreaked onto SD -Ura +NAT petri plates, to attain single colonies with the appropriate selection markers.

\subsubsection{Confirmation of resistance by serial spot dilution}

Single colonies from each mutant (so named from here on as TA1-TA22 and EQ1EQ21), as well as the parental strain used to generate resistant mutants, $p d r \Delta$, were inoculated into $2 \mathrm{~mL} \mathrm{SC}$ liquid media and grown overnight at $30^{\circ} \mathrm{C}$. Cells were then collected by brief centrifugation, washed twice, and resuspended in $d \mathrm{dH}_{2} \mathrm{O}$. A $100 \mu \mathrm{L}$ aliquot from each culture was dispensed to a 96-well plate, and the OD at $590 \mathrm{~nm}$ was measured to gauge the cell concentration of each culture. Where appropriate, cell cultures were diluted to give $1 \times 10^{8}$ cells $/ \mathrm{mL}$. These were then serially diluted ten-fold, thrice, in 96-well plates, from which $1.5 \mu \mathrm{L}$ of each cell diluent was inoculated on to SC + NAT containing either $60 \mu \mathrm{M}$ TA-289 or $60 \mu \mathrm{M}$ equisetin, giving final cell concentrations of 150 000, 15000 , and 1500 cells per well, respectively.

All mutant strains and the parental strain $(p d r \Delta)$ were tested against both compounds to not only confirm resistance, but also to identify the possibility of 
cross-resistance. Non-drug containing plates (SC +NAT only) served as carrier controls. Excess moisture was removed by drying the plates in a laminar flow hood, and then incubated at $30^{\circ} \mathrm{C}$ for three days, after which they were photographed and colony sizes scored according to the level of resistance compared to controls; a score of 1 = little growth (weak resistance), 2 = fair growth, 3 = good growth, 4 = very good growth (strong resistance, grows like parental strain $(p d r \Delta)$ in untreated conditions).

\subsubsection{Identifying mutant phenotypes under differing stress conditions}

Briefly, cell concentrations of overnight cultures of all confirmed resistant mutants (TA1-22, EQ1-21), as well as the parental strain used to generate the mutants, $p d r \Delta$, were determined and where necessary diluted to give $1 \times 10^{8}$ cells $/ \mathrm{mL}$. These mutants were transferred to a 96-well tissue culture plate and arrayed in quadruplicates in 384 format on a Singer ${ }^{\odot}$ plate using a Singer RoToR $^{\odot}$ HDA (Singer Instruments Co. Ltd., Somerset, UK), with the parental strain bordering the arrayed mutants. The mutant and parental cultures were then further arrayed onto Singer ${ }^{\odot}$ plates containing either SC, SC-Gly-EtOH (SC containing 2\% glycerol and 2\% ethanol, instead of glucose), SC-Gal (SC containing 2\% galactose, instead of glucose), $\mathrm{SC}+50 \mathrm{nM}$ cycloheximide, or SC + 0.4 mM DTT. The plates were then incubated at three different temperatures; $25^{\circ} \mathrm{C}, 30^{\circ} \mathrm{C}$, and $37{ }^{\circ} \mathrm{C}$ for two days. Photographs of the plates were taken using ZoomBrowser EX remote shooting software (Canon) after two days and analysed by Colony HT (Collins, Schuldiner et al. 2006). 


\subsubsection{Creating an isogenic wild-type for mating with resistant mutants}

\subsubsection{PCR amplification of the URA3 deletion cassette}

Primers used for this PCR were as follows:

\begin{tabular}{|l|l|l|}
\hline Primer \# & Primer spec & Primer Sequence \\
\hline 353 & PDR3 forward deletion & $\begin{array}{l}\text { ACTGCATCAGCAGTTTTATTAATTTTT } \\
\text { TCTTATTGCGTGACCGCAACATGGAG } \\
\text { GCCCAGAATACCCT }\end{array}$ \\
\hline 354 & PDR3 reverse deletion & $\begin{array}{l}\text { CCATTTACTATGGTTATGCTCTGCTTC } \\
\text { CCTATTTCTTTTGCGTTTCAGTATAGC } \\
\text { GACCAGCATTCAC }\end{array}$ \\
\hline
\end{tabular}

Briefly, $5 \mu \mathrm{L}$ of purified pAG60 plasmid (containing the $U R A 3$ gene) was added to a PCR tube containing $20 \mu \mathrm{L}$ of PCR reagent mix $(14.15 \mu \mathrm{L}$ ddH2O, $5 \mu \mathrm{L}$ Q buffer (Qiagen), $2.5 \mu \mathrm{L}$ 10x PCR buffer, $1 \mu \mathrm{L} 20 \mathrm{mM}$ dNTPs (5 mM each dNTP), $0.75 \mu \mathrm{L}$ $50 \mathrm{mM} \mathrm{MgCl} 2,0.25 \mu \mathrm{L} 100 \mathrm{mM}$ forward deletion primer, $0.25 \mu \mathrm{L} 100 \mathrm{mM}$ reverse deletion primer, $0.1 \mu \mathrm{L}$ Hot Star taq). A second tube containing no template served as a negative control. Reaction controls for the PCR were: $94{ }^{\circ} \mathrm{C}$ for 15 mins for enzyme activation and template denaturation, 35 cycles of $95{ }^{\circ} \mathrm{C}$ for $45 \mathrm{~s}$ for template denaturation, $54{ }^{\circ} \mathrm{C}$ for $40 \mathrm{~s}$ for primer annealing, $68{ }^{\circ} \mathrm{C}$ for $1 \mathrm{~min}$ for primer extension, and $68^{\circ} \mathrm{C}$ for 10 mins to complete any partially extended primers. PCR products were kept at $10{ }^{\circ} \mathrm{C}$ to prevent heat denaturation. A $5 \mu \mathrm{L}$ aliquot of the PCR product and an aliquot of the purified pAG60 plasmid were run on a $1 \%$ agarose gel stained with ethidium bromide at $100 \mathrm{~V}$ for 25 mins, after which the gel 
was visualized under UV, a photo taken of the gel and expected PCR product band sizes determined according to the $1 \mathrm{~kb}$ plus ladder (Invitrogen NZ ltd). Expected band size for the pAG60 plasmid was $3944 \mathrm{bp}$, and for the $U R A 3$ deletion cassette PCR product, $\sim 1600 \mathrm{bp}$.

\subsubsection{High efficiency transformation with URA3 deletion cassette}

An overnight culture of a MATa $p d r 1 \Delta:: k a n R(p d r 1 \Delta-\mathrm{p})$ strain was grown in $2 \mathrm{~mL}$ SC media at $30^{\circ} \mathrm{C}$, after which the cell concentration of the culture was determined by measuring OD at $600 \mathrm{~nm}$ using a spectrophotometer (Genova Life Science), and subsequently diluted to give $1 \times 10^{7}$ cells/mL in a $15 \mathrm{~mL}$ volume. The culture was then incubated in a shaking incubator at $30{ }^{\circ} \mathrm{C}$ for a further $7 \mathrm{~h}$ to ensure a cell culture at mid-log phase (as determined by counting on a haemocytometer). Following the high-efficiency transformation protocol (Gietz and Schiestl 2007), the cells were transformed with the URA3 deletion cassette amplified with deletion primers with homology to both the $U R A 3$ cassette and to the flanking regions of the $P D R 3$ open reading frame to generate a MATa $p d r 1 \Delta:: k a n R$ pdr3A::URA3 strain (referred here on as YCG387).

Transformants were streaked onto SD -Ura +G418 plates to obtain single colonies for PCR confirmation. The same colony used for PCR confirmation was used to inoculate $10 \mathrm{~mL}$ YPD media and grown overnight at $30{ }^{\circ} \mathrm{C}$ in a shaking incubator to 
stationary phase, after which the cells were collection by centrifugation, washed, and resuspended in $1 \mathrm{~mL}$ YP with $15 \%$ glycerol. The $1 \mathrm{~mL}$ culture was then transferred to a cryotube and stored in $-80^{\circ} \mathrm{C}$ until further use.

\subsubsection{Confirmation of transformants by colony $P C R$}

Two transformant colonies and the $p d r 1 \Delta-\mathrm{p}$ parental strain used for this transformation (to serve as a negative control) were incubated in $50 \mu \mathrm{L} 1 \mathrm{mg} / \mathrm{mL}$ zymolase in $\mathrm{ddH}_{2} \mathrm{O}$ at $30{ }^{\circ} \mathrm{C}$ for 30 mins. A $5 \mu \mathrm{L}$ aliquot from each of the cellzymolase suspension was added to the PCR tubes; pair tubes were made for each cell-zymolase suspension. Into each tube $20 \mu \mathrm{L}$ PCR reagent mix (containing $162 \mu \mathrm{L}$ ddH ${ }_{2} \mathrm{O}, 25 \mu \mathrm{L}$ 10x PCR buffer, $8 \mu \mathrm{L} 20$ mM dNTPs (5 mM each dNTP), $2 \mu \mathrm{L} 100 \mathrm{mM}$ forward deletion primer, $2 \mu \mathrm{L} 100 \mathrm{mM}$ reverse deletion primer, $1 \mu \mathrm{L}$ Hot Star taq) containing the appropriate primers were added. Primers used were:

\begin{tabular}{|l|l|l|l|}
\hline Primer & Primer spec & Primer Sequence & $\begin{array}{l}\text { Expected } \\
\text { size }\end{array}$ \\
\hline 368 & URA3 forward internal & GACACCTGGAGTTGGATT & \multirow{2}{*}{$880 \mathrm{bp}$} \\
\hline 369 & $U R A 3$ reverse external & TTATGAACACGCACAGGC & \\
\hline 370 & URA3 forward external & AATTCAACGCGTCTGTGAGG & \multirow{2}{*}{$761 \mathrm{bp}$} \\
\cline { 1 - 3 } 371 & URA3 reverse internal & TACCGCCTAGGTAACCAT & \\
\hline
\end{tabular}

Reaction controls for the PCR were: $94{ }^{\circ} \mathrm{C}$ for 15 mins for enzyme activation and template denaturation, 35 cycles of $95{ }^{\circ} \mathrm{C}$ for $45 \mathrm{~s}$ for template denaturation, $54{ }^{\circ} \mathrm{C}$ 
for $40 \mathrm{~s}$ for primer annealing, $68^{\circ} \mathrm{C}$ for $1 \mathrm{~min}$ for primer extension, and $68^{\circ} \mathrm{C}$ for 10 mins to complete any partially extended primers. PCR products were kept at $10{ }^{\circ} \mathrm{C}$ to prevent heat denaturation. A $5 \mu \mathrm{L}$ aliquot of each PCR product were run on a $1 \%$ agarose gel stained with ethidium bromide at $100 \mathrm{~V}$ for 25 mins, after which the gel was visualized under UV, a photo taken of the gel and expected PCR product band sizes determined according to the $1 \mathrm{~kb}$ plus ladder (Invitrogen).

\subsubsection{Determining dominance and/or recessiveness of resistant mutants}

\subsubsection{Generating heterozygous diploid mutants}

Briefly, the strongest confirmed resistant mutant, TA8, as well as the parental strain used to generate the resistant mutants (YCG326, pdr $\Delta$ ) were mated with the isogenic MATa wild-type strain (YCG387) and then replica-plated onto SD -Ura +NAT +G418 to select for diploids, namely, the mutants that have successfully mated with the isogenic wt strain. The desired diploids were re-streaked onto fresh SD -Ura $+\mathrm{NAT}+\mathrm{G} 418$ petri plates and grown for two days at $30^{\circ} \mathrm{C}$ to attain single colonies. These heterozygous diploids were used to determine dominance and recessiveness of the mutations.

Cell concentrations of overnight cultures of TA8, pdrA parental, along with the corresponding heterozygous diploids were determined by measuring OD at $600 \mathrm{~nm}$ 
on a spectrophotometer (Genova Life Science), and subsequently diluted to $5 \times 10^{5}$ cells/mL. Each yeast culture was then serially diluted ten-fold, four times, after which a $10 \mu \mathrm{L}$ aliquot from each dilution was spotted onto petri plates containing $60 \mu \mathrm{M}$ TA-289, as well as the corresponding untreated control plates containing only SC media. Excess moisture was removed by drying the plates in a laminar flow hood, incubated for two days at $30^{\circ} \mathrm{C}$, and then photographed. The level of resistance was determined by colony growth of heterozygous diploids mutants to original haploid resistant mutants on drug-containing plates.

\subsubsection{Growth rate assays}

Overnight cultures of $p d r \Delta$ and of the TA8 resistant mutant were prepared by inoculating into $2 \mathrm{~mL}$ SC media and subsequently incubated overnight at $30^{\circ} \mathrm{C}$. Cell concentrations were determined by measuring $\mathrm{OD}$ at $600 \mathrm{~nm}$ on a spectrophotometer (Genova Life Science), and the cultures diluted to $2 \times 10^{7}$ cells $/ \mathrm{mL}$ in fresh SC media. A $500 \mu \mathrm{L}$ aliquot of each diluted culture was dispensed in triplicate into the centre wells of a 48-well tissue culture plate, and surrounding these, wells containing $500 \mu \mathrm{L}$ of $\mathrm{ddH}_{2} \mathrm{O}$ throughout the entire plate. The plate was then placed in the Wallac EnVision 2102 Multilabel Plate Reader (Perkin Elmer ${ }^{\mathrm{TM}}$, Waltham, Mass.) at $30^{\circ} \mathrm{C}$. The plate was mixed and absorbance read at OD $590 \mathrm{~nm}$ every 15 mins for $18 \mathrm{~h}$. Raw OD data obtained were natural log (ln) transformed and fitted with the LINEST function in Microscoft Excel (2007) to a straight line, which gave estimates of the gradient and y-intercept. These were used 
to calculate growth rates of both strains. See appendix 2.3 for full calculations of doubling-time for each strain.

\subsubsection{Proof-of-principle C-SGAM with microarray: Using cycloheximide as a pilot}

A $10 \mathrm{~mL}$ overnight culture of YCG191, a cycloheximide resistant mutant $\left(\mathrm{CYH}^{\mathrm{r}}\right)$, was divided and $100 \mu \mathrm{L}$ aliquots transferred to a 96-well tissue culture plate and arrayed in 384 format onto YPD +NAT Singer ${ }^{\odot}$ plates. Quadruplicate plates were arrayed and incubated at $30{ }^{\circ} \mathrm{C}$ for two days in preparation for mating with the homozygous haploid MATa deletion set. SGA analysis was carried out using the Singer RoToR ${ }^{\odot}$ HDA (Singer Instruments Co. Ltd., Somerset, UK) following the protocol set out by Tong and Boone 2006.

Duplicate sets of the second-to-last selection stage of the SGA (SD -His/Arg/Lys +Can +Thia +G418) were generated, one set of which was subsequently pinned onto SD -His/Arg/Lys +Can +Thia +G418 +10 $\mu \mathrm{g} / \mathrm{mL}$ cycloheximide.

For the proof-of-principle mapping by microarray, the border colonies of the second set of plates were removed and the remaining colonies from each plate were pooled and $15 \mathrm{~mL}$ aliquots were freeze dried and stored at $-80{ }^{\circ} \mathrm{C}$ (Mike Cook, School of Biological Sciences, University of Edinburgh, UK, personal communication). A 500 $\mu \mathrm{L}$ aliquot of the pooled cells was used to inoculate $10 \mathrm{~mL}$ fresh $\mathrm{SC}+\mathrm{G} 418$ and incubated overnight at $30{ }^{\circ} \mathrm{C}$ on a rotating drum. Cell concentrations of the overnight cultures were determined by measuring $O D$ at $600 \mathrm{~nm}$ on a 
spectrophotometer (Genova Life Science), and subsequently diluted to $1 \times 10^{7}$ cells $/ \mathrm{mL}$ in $10 \mathrm{~mL}$ fresh SC + G418. A $100 \mu \mathrm{L}$ aliquot of $1 \mathrm{mg} / \mathrm{mL}$ cycloheximide was added to the yeast culture, giving a final concentration of 10 $\mu \mathrm{g} / \mathrm{mL}$ cycloheximide. DMSO (1\% final concentration) served as a carrier control. The cultures were mixed by brief vortexing, and incubated at $30{ }^{\circ} \mathrm{C}$ on a rotating drum for $15 \mathrm{~h}$.

Following the first overnight incubation, the cultures were further diluted to $1 \times 10^{7}$ cells/mL in $10 \mathrm{~mL}$ fresh $\mathrm{SC}+\mathrm{G} 418$, and treated with the same concentration of cycloheximide or DMSO, then incubated for another $15 \mathrm{~h}$ at $30{ }^{\circ} \mathrm{C}$. Genomic DNA of both treated and untreated cultures were precipitated, purified, quantified, amplified, and hybridized onto arrays following the MasterPure DNA Purification Kit (Epicentre Biosciences) and the yeast tag microarray protocol (Rosemary Heathcott, personal communication, ESR Wellington) adapted from Parsons 2006.

\subsubsection{Microarray analysis}

The microarray chips were read on an Axon Microarray Scanner, where fluorescently labeled control tags were scanned at $532 \mathrm{~nm}$ and experimental samples (drug-treated) scanned at $635 \mathrm{~nm}$ (Genomics Facility, Biochemistry Department, Otago University). 
The intensities of the up and down tags were normalized and $\log _{2}$ ratios of drugtreated/DMSO control data were obtained using Standardization and Normalization of Microarray Data (SNOMAD) software (http://www.pevsnerlab. kennedykrieger.org/snomadinput.html). Gene tags with a low fluorescent control signal (less than 500) were removed, the remaining log ratios of tag replicates were averaged, and deviation (D) scores calculated $(D$ score $=$ (average of log ratios - mean of average of $\log$ ratios)/standard deviation of average of log ratios). Gene tags were ordered according to chromosome position and the region surrounding the RPL28 locus of resistance was plotted against the respective $\log _{2}$ ratios.

\subsubsection{Reactive oxygen species detection by flow cytometry}

Cell concentrations of overnight cultures of $p d r \Delta$ and the haploid TA-289 resistant mutant, TA8, were determined by measuring $\mathrm{OD}$ at $600 \mathrm{~nm}$, and subsequently diluted to $1 \times 10^{7}$ cells/mL in fresh SC media. pdr $\Delta$ cells were treated with $\mathrm{H}_{2} \mathrm{O}_{2}$ (1 mM final concentration) and incubated for 30 mins at $30{ }^{\circ} \mathrm{C}$. Following each incubation period, cells were treated with DCF (50 $\mu \mathrm{M}$ final concentration) and further incubated at $30{ }^{\circ} \mathrm{C}$ for 15 mins. Cells were then placed on ice, diluted with

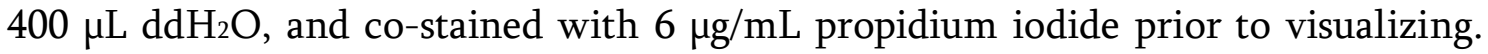
The flow cytometry data obtained were analysed on FlowJo version 7.6.1 flow cytometry analysis software (Tree Star Inc., Ashland, OR, USA). Cell populations 
were manually gated according to the untreated control and recorded as percentage of total population within the sample.

\subsubsection{Cell death assays}

Overnight cultures of $p d r \Delta$ and TA8 were prepared by inoculating either $2 \mathrm{~mL}$ SC, or $2 \mathrm{~mL} \mathrm{SC}+\mathrm{gly}-\mathrm{EtOH}$, and grown overnight at $30{ }^{\circ} \mathrm{C}$ on a rotating drum. Cell concentrations of each culture were determined by measuring OD at $600 \mathrm{~nm}$ and subsequently diluted in either fresh SC or SC + gly-EtOH to give a final cell concentration of $1 \times 10^{6}$ cells $/ \mathrm{mL}$.

For the TA8 and pdr $\Delta$ cell death assays in $\mathrm{H}_{2} \mathrm{O}_{2}$, an eight-point serial dilution of $\mathrm{H}_{2} \mathrm{O}_{2}$ was prepared in $\mathrm{ddH}_{2} \mathrm{O}$ to give a concentration range from $0.9 \mathrm{M}$ to $0.3 \mathrm{M}$. A $100 \mu \mathrm{L}$ aliquot of diluted cells in either SC or SC +gly-EtOH were dispensed into 96well tissue culture plates, into which $1 \mu \mathrm{L}$ aliquots of each $\mathrm{H}_{2} \mathrm{O}_{2}$ diluent were added, giving final concentrations of $9 \mathrm{mM}$ to $3 \mathrm{mM}$. After addition of $\mathrm{H}_{2} \mathrm{O}_{2}$, the plates were mixed by vortexing, and a $1.5 \mu \mathrm{L}$ aliquot of the treated cells were removed and spotted onto a Singer ${ }^{\odot}$ plate containing SC. DMSO (1\% final concentration) served as a carrier control for both assays. The plates were then incubated at $30{ }^{\circ} \mathrm{C}$. A $1.5 \mu \mathrm{L}$ aliquot of cells were harvested and spotted onto the SC plate every 30 mins for $3 \mathrm{~h}$ for cells in SC, and every 30 mins after the first hour for $2.5 \mathrm{~h}$ for cells in SC +gly$\mathrm{EtOH}$. 


\subsubsection{C-SGAM of TA-289 resistant mutant by hydrogen peroxide}

A $10 \mathrm{~mL}$ overnight culture of the TA-289 resistant mutant, TA8, was transferred to a 96-well tissue culture plate $(100 \mu \mathrm{L}$ aliquots per well), and then arrayed in 384 format onto SC Singer ${ }^{\odot}$ plates. Triplicate plates were arrayed and incubated at $30{ }^{\circ} \mathrm{C}$ for two days in preparation for mating with the homozygous haploid MATa deletion set. SGA analysis was carried out using the Singer RoToR ${ }^{\odot}$ HDA (Singer Instruments Co. Ltd., Somerset, UK) following the protocol set out by Tong and Boone 2006 with slight modifications; After the haploid selection step, cells were twice treated with $6 \mathrm{mM} \mathrm{H} \mathrm{H}_{2}$ prior to the final selection with $\mathrm{G} 418$. 


\section{$\underline{3.6 \text { Results }}$}

\subsubsection{Resistant mutant generation}

Mutants resistant to TA-289 and equisetin were generated by exposure to EMS and also by spontaneous mutations. A total of 22 spontaneous and EMS-generated mutants resistant to TA-289 (TA1-TA22), and 21 resistant to equisetin (EQ1-EQ21) were collected. These mutants were confirmed by a serial-spot dilution with $60 \mu \mathrm{M}$ TA-289 or equisetin. In addition, the mutants were also tested for cross-resistance by testing TA mutants on $60 \mu \mathrm{M}$ equisetin, and EQ mutants on $60 \mu \mathrm{M}$ TA-289. All 43 mutants were also grown in the presence of xenobiotics $(1 \mathrm{mg} / \mathrm{mL}$ cycloheximide (CYH), $0.4 \mathrm{mM} \mathrm{DTT})$, or in different carbon sources ( $2 \%$ galactose and $2 \%$ glyEtOH), and all were subjected to normal $\left(30^{\circ} \mathrm{C}\right.$, ) high $\left(37^{\circ} \mathrm{C}\right)$, and sub-optimum $\left(25^{\circ} \mathrm{C}\right)$ temperature conditions. Identifying conditional mutants provides an insight into the mutation itself, in addition, it may provide a growth-restrictive condition to be used in the mapping procedure instead of using a small molecule inhibitor (i.e. TA-289 and equisetin) as a probe.

Of the 22 TA-289 resistant mutants, five were cross-resistant to equisetin. Of the 21 equisetin resistant mutants, six were cross-resistant to TA-289 (a score of $>2$ was deemed resistant, where a score of 4 was considered strong resistance. See appendix 2.1 for table of scores and figure of mutants) (Figure 3.3). When tested under different stress conditions, six of the 43 mutants displayed differential sensitivity 
compared to the parental strain $p d r \Delta$ when galactose (Gal) or glycerol-ethanol (Gly$\mathrm{EtOH}$ ) was used as a carbon source. One mutant, EQ2, did not grow in Gal or Gly$\mathrm{EtOH}$ at $25{ }^{\circ} \mathrm{C}$ and $37^{\circ} \mathrm{C}$, but was sensitive only to $\mathrm{Gly}$-EtOH at $30{ }^{\circ} \mathrm{C}$. No mutants displayed altered sensitivity to CYH or DTT at any tested temperatures (Table 3.1).

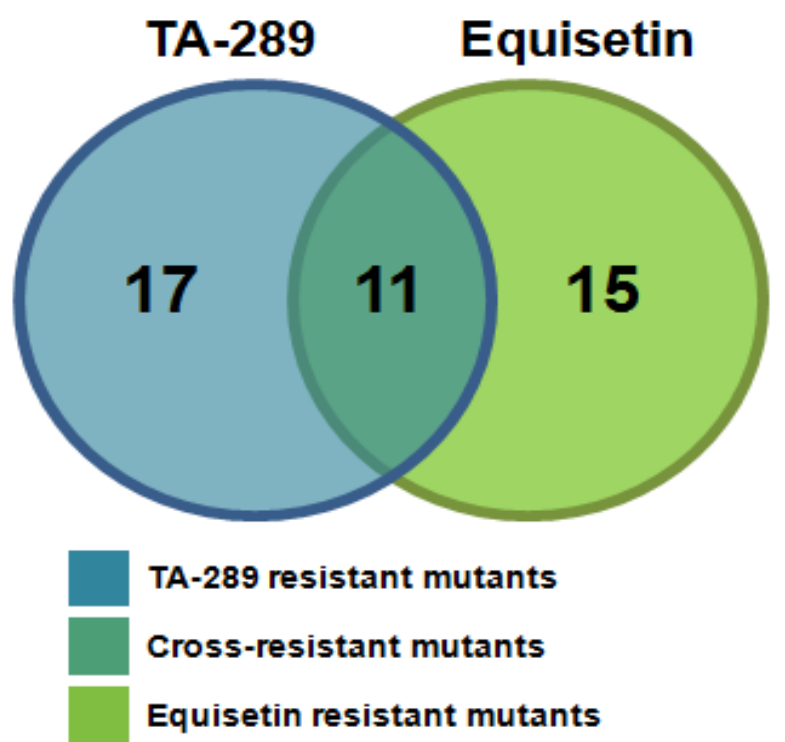

Figure 3.3. TA-289 and equisetin resistant mutants. This Venn diagram illustrates the number of mutants generated from either spontaneous mutations or from EMS-induced mutations resistant to TA-289 and equisetin, as well as the cross-resistant mutants.

\begin{tabular}{|c|c|c|c|c|c|c|c|c|c|c|c|c|c|c|}
\hline \multirow[b]{2}{*}{ Mutant } & \multicolumn{3}{|c|}{$25^{\circ} \mathrm{C}$} & \multicolumn{6}{|c|}{$30^{\circ} \mathrm{C}$} & \multicolumn{4}{|c|}{$37^{\circ} \mathrm{C}$} & \multirow[b]{2}{*}{ Score } \\
\hline & CYH DTT & Gal & $\begin{array}{l}\text { Gly- } \\
\text { EtOH }\end{array}$ & & CYH DT & & $\mathrm{Gal}$ & $\begin{array}{l}\text { Gly- } \\
\text { EtOH }\end{array}$ & & $\mathrm{CYH}$ & DTT & $\mathrm{Gal}$ & $\begin{array}{l}\text { Gly- } \\
\text { EtOH }\end{array}$ & \\
\hline EQ2 & 0 & 0 & 1 & 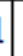 & 0 & 0 & 0 & & 1 & 0 & & 0 & 1 & 1 \\
\hline TA13 & 0 & 0 & 0 & 1 & 0 & 0 & 1 & & 1 & 0 & & 0 & 1 & 0 \\
\hline TA4 & 0 & 0 & 1 & ) & 0 & 0 & 1 & & 0 & 0 & & 0 & 1 & 0 \\
\hline EQ11 & 0 & 0 & 1 & 1 & 0 & 0 & 0 & & 0 & 0 & & 0 & 1 & 0 \\
\hline TA6 & 0 & 0 & 0 & D & 0 & 0 & 1 & & 1] & 0 & & 0 & 0 & 0 \\
\hline $\mathrm{EQ} 3$ & 0 & 0 & 0 & 1 & 0 & 0 & 0 & & 0 & 0 & & 0 & 1 & 0 \\
\hline
\end{tabular}

Table 3.1. Table describing resistant mutants that display differential sensitivity under varying stress conditions compared to the parental strain. Growth/no growth in each condition was determined by scoring either a 0 (normal growth), or 1 (no growth) for each mutant. Each score of 1 indicates sensitivity to the particular condition compared to parental growth. An overall score indicates sensitivity of each mutant to the conditions tested. 


\subsubsection{Mutation in TA8 is dominant}

Almost all but 4 mutants displayed only transient resistance, and so lost resistance to TA-289 and/or equisetin over time. One mutant, TA8, was chosen for further study as it displayed strongest resistance and was not as slow-growing as the other mutants. TA8 did not display cross-resistance to equisetin, nor was it sensitive in any of the conditions tested. To determine whether the mutation in TA8 is dominant or recessive, TA8 was mated to an isogenic wild-type strain YCG387 (see appendix 2.2), and both haploid and heterozygous diploid strains were tested with $60 \mu \mathrm{M}$ TA-289, the concentration that was originally used for resistant mutant generation. The heterozygous diploid mutant was not growth inhibited by TA-289, suggesting that the mutation in TA8 is dominant (Figure 3.4).

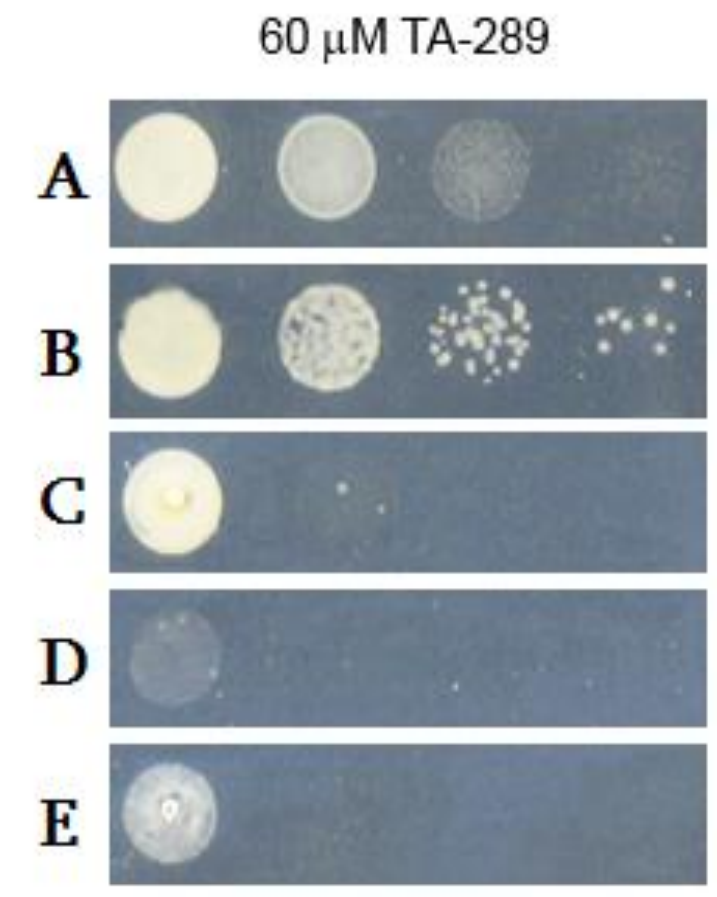

Figure 3.4. Mutation in TA8 is dominant. TA8 (A), the corresponding heterozygous diploid (B), the parental wt haploid strain used to generate the resistant mutant $(C)$, the isogenic wt haploid strain used to mate with TA8 (D), and the corresponding wt heterozygous diploid (E) were serially diluted four-fold and spotted onto $60 \mu \mathrm{M}$ TA-289. 


\subsubsection{Proof-of-principle C-SGAM with microarray}

A pilot C-SGAM with a microarray step at the final was conducted using a $\mathrm{CYH}^{\mathrm{r}}$ mutant. C-SGAM with this mutant was successfully performed by Ploi Yibmantasiri (School of Biological Sciences, Victoria University of Wellington), however measuring growth alterations from pooled deletion mutants in competitive growth assays using the microarray method at the end of the SGA procedure is novel. The $\mathrm{CYH}^{\mathrm{r}}$ mutant was chosen because the locus of resistance is known (rpl28Q208L), allowing us to identify it both on the agar plates as well as in the microarray. A linkage disequilibrium pattern was indeed visible on the expected location in the SGA, where non-essential genes in linkage disequilibrium with RPL28 (YGL103W) show a pattern of growth defect, with the greatest growth defects occurring in genes directly adjacent to the locus of RPL28. (Figure 3.5).

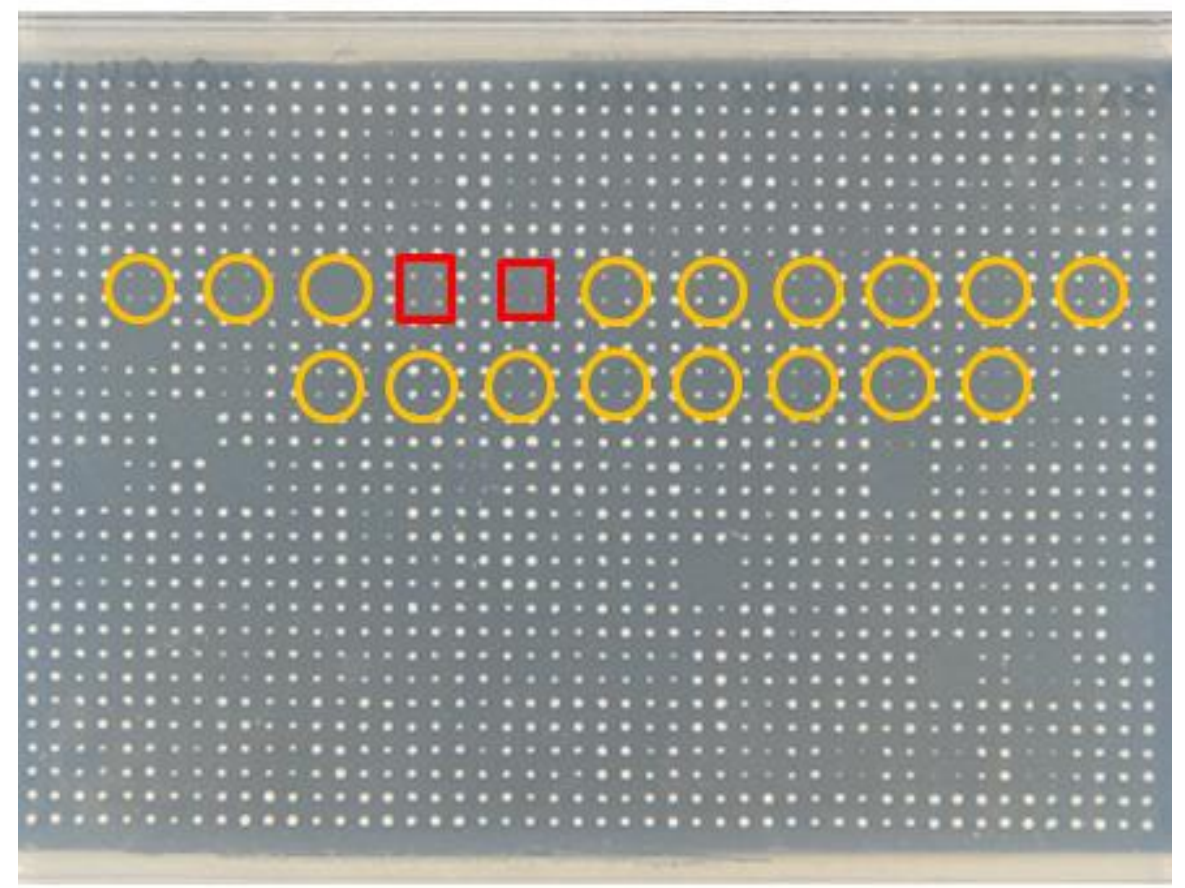

Figure 3.5. Linkage disequilibrium pattern around RPL28 locus. Yellow circles show linkage pattern, where the genes adjacent to RPL28 (red squares), YGL104C and YGL101W showed the greatest growth defect. 
This linkage pattern was not observed in the microarray growth assay. Strains deleted for genes in linkage disequilibrium with $R P L 28$ were expected to be underrepresented (the more negative the $\log _{2}$ ratio value, the more under-represented a given gene) compared to the control, however the $\log _{2}$ ratio of the fluorescence intensities of both up (UP) and down (DN) tags of the open reading frames (ORF) of genes adjacent to RPL28 (YGL104C and YGL101W) showed no difference in intensity compared to the rest of the deletion strains identified in the microarray (figure 3.6).

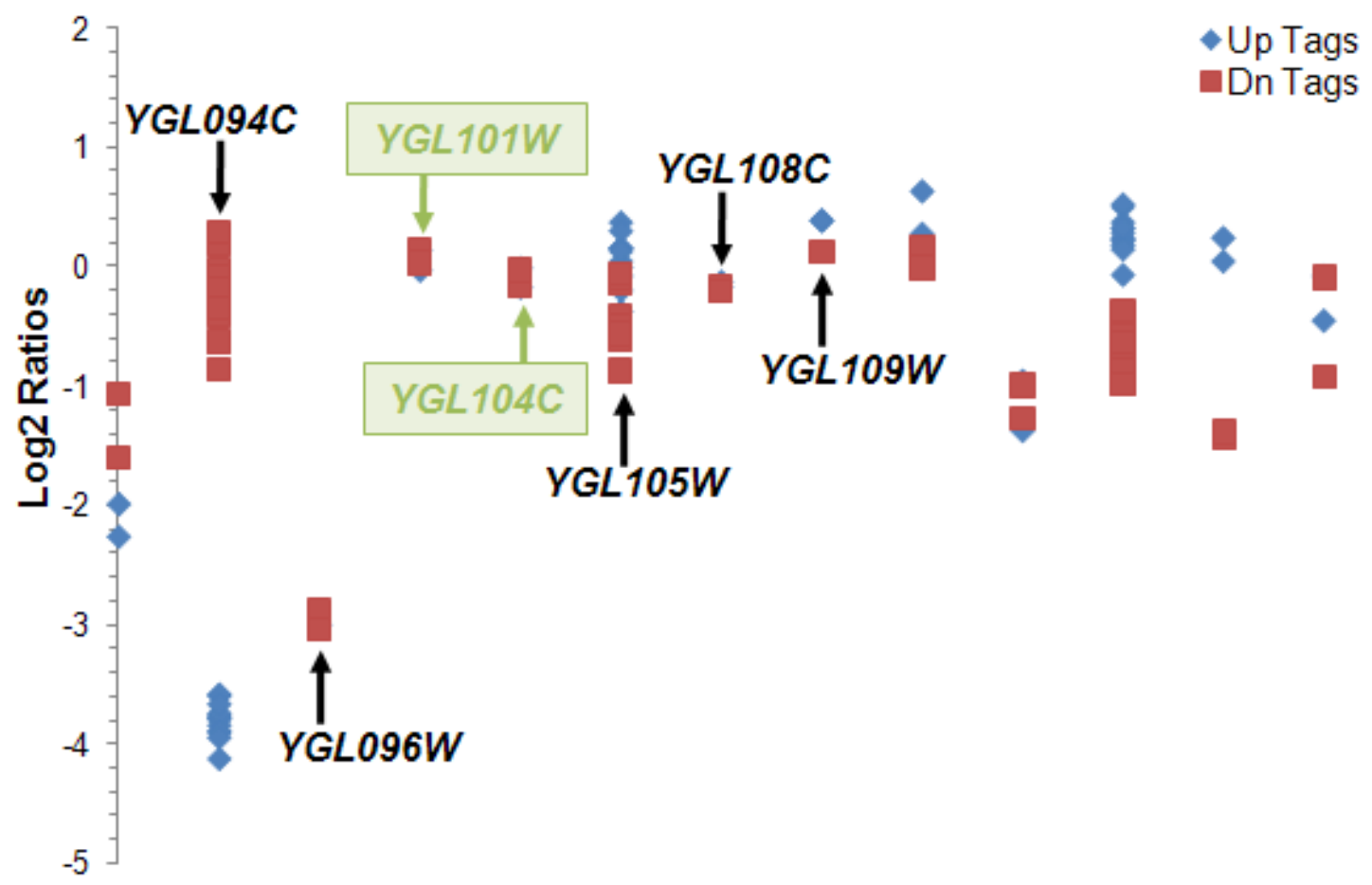

Figure 3.6. A close-up view of neighbouring genes to RPL28. Log2 ratios of both UP and DN tags of the chromosome region corresponding to RPL28 and its neighbouring genes are plotted. Genes adjacent to RPL28, YGL101W and YGL104C (green boxes) are as noted. Neighbouring genes along the chromosome are also noted in black. 


\subsubsection{TA8 mutant displays resistance by upregulating anti-oxidant production}

In combination with the results from chapter two indicating a possible target at the mitochondria for TA-289 and equisetin, and also from previously reported activities suggesting that equisetin acts on the mitochondrial respiratory chain (König, Kapus et al. 1993), an involvement of reactive oxygen species (ROS) was thought to be a likely possibility. Thus, the TA8 mutant was tested for its ability to grow in the presence of TA-289 and equisetin under different carbon sources, as well as the level of intracellular ROS production of the mutant. The oxidant-sensitive fluorescent dye 2,7-dichlorofluoroscein diacetate (DCF) was used to quantitatively measure ROS production within a cell. Propidium iodide was used as a counter stain to detect cells with compromised cell membranes, often indicated as dead cells. $\mathrm{H}_{2} \mathrm{O}_{2}$ treatment served as a positive control for ROS production. Interestingly, untreated TA8 mutant cells displayed a higher basal level of ROS production than the untreated $p d r \Delta$ parental cells, and also displaying more ROS production in an untreated state compared to when $p d r \Delta$ cells were treated with $\mathrm{H}_{2} \mathrm{O}_{2}$ (Figure 3.7). 


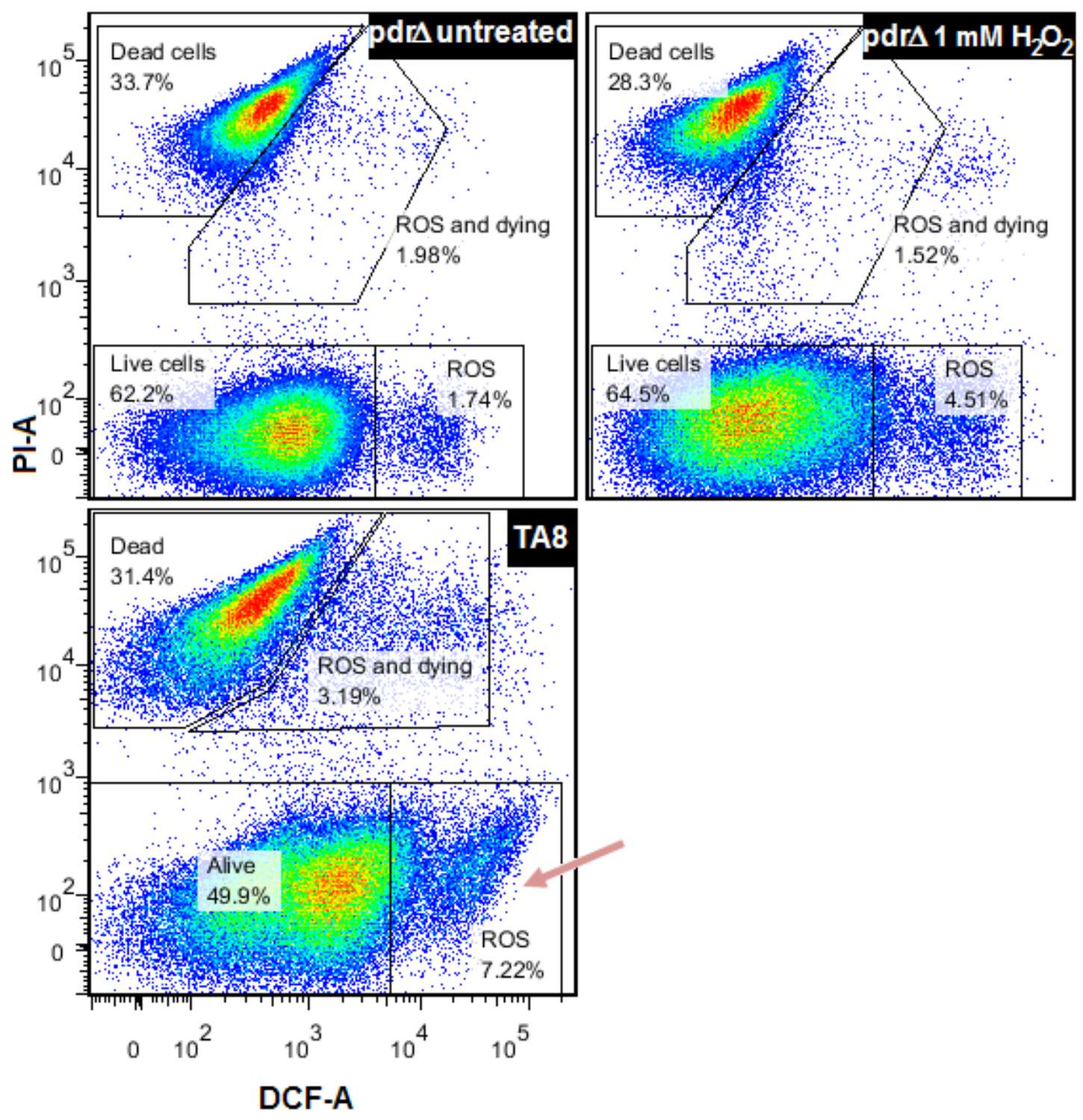

Figure 3.7. TA8 mutant displays a higher basal level of ROS production. pdr $\Delta$ cells were either treated with $1 \mathrm{mM} \mathrm{H} \mathrm{H}_{2} \mathrm{O}_{2}$ (top right: $p d r \Delta 1 \mathrm{mM} \mathrm{H} \mathrm{H}_{2}$ ), or left untreated (Top left: $p d r \Delta$ untreated), and the TA8 mutant untreated (Bottom left: TA8), were stained with DCF ( $x$-axis) to detect ROS, and co-stained with propidium iodide (y-axis) to detect dead cells. Cell populations were gated according to the untreated $p d r \Delta$ control. The pink arrow highlights the increase in ROS level observed in the TA8 mutant under normal untreated conditions. 
As it was suggested that the resistance of the TA8 mutant to TA-289 was due to its higher basal level of ROS production, and thus presumably an overall greater antioxidant capacity, a cell death assay of the TA8 mutant treated with varying concentrations of a known oxidant $\mathrm{H}_{2} \mathrm{O}_{2}$ was performed to truly determine whether this was a specific mutation or a non-specific mutation that allows for the mutant to be resistant to different oxidants. Figure 3.8 shows that TA8 is resistant to $\mathrm{H}_{2} \mathrm{O}_{2}$ even at the highest concentration $(9 \mathrm{mM})$, while the parental $p d r \Delta$ cells were much more sensitive.

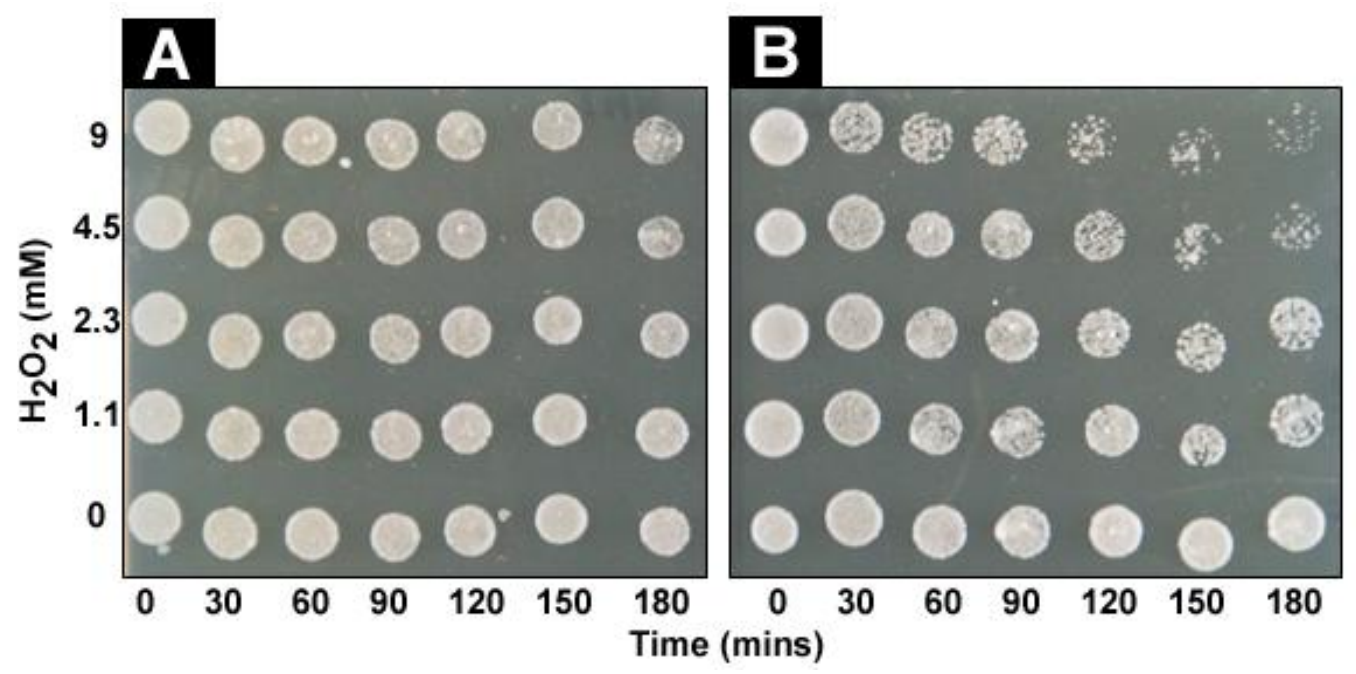

Figure 3.8. TA8 is resistant to $\mathrm{H}_{2} \mathrm{O}_{2}$. TA8 (A) and $p d r \Delta$ (B) cells were treated with varying concentrations of $\mathrm{H}_{2} \mathrm{O}_{2}$ (y-axis) with glucose as a carbon source and incubated at $30{ }^{\circ} \mathrm{C}$ for $3 \mathrm{~h}$. An aliquot of cells treated at each concentration were spotted onto SC plate every 30 mins ( $\mathrm{x}$ axis) and incubated for 2 days at $30^{\circ} \mathrm{C}$.

This suggests that the mechanism of resistance of the TA8 mutant to TA-289 is a result of a non-oxidant specific mutation that caused an overall higher production of ROS, and thus an overall upregulation of anti-oxidants to adapt to the ROS 
production within the cell, and more importantly, ROS induced by TA-289 or other oxidants such as $\mathrm{H}_{2} \mathrm{O}_{2}$. Interestingly, TA8 was more sensitive to $\mathrm{H}_{2} \mathrm{O}_{2}$ when glycerolethanol was used as a carbon source compared to the $p d r \Delta$ parental strain (Figure 3.9), suggesting a possible threshold of antioxidant capacity that may be a result of the resistance-conferring mutation.

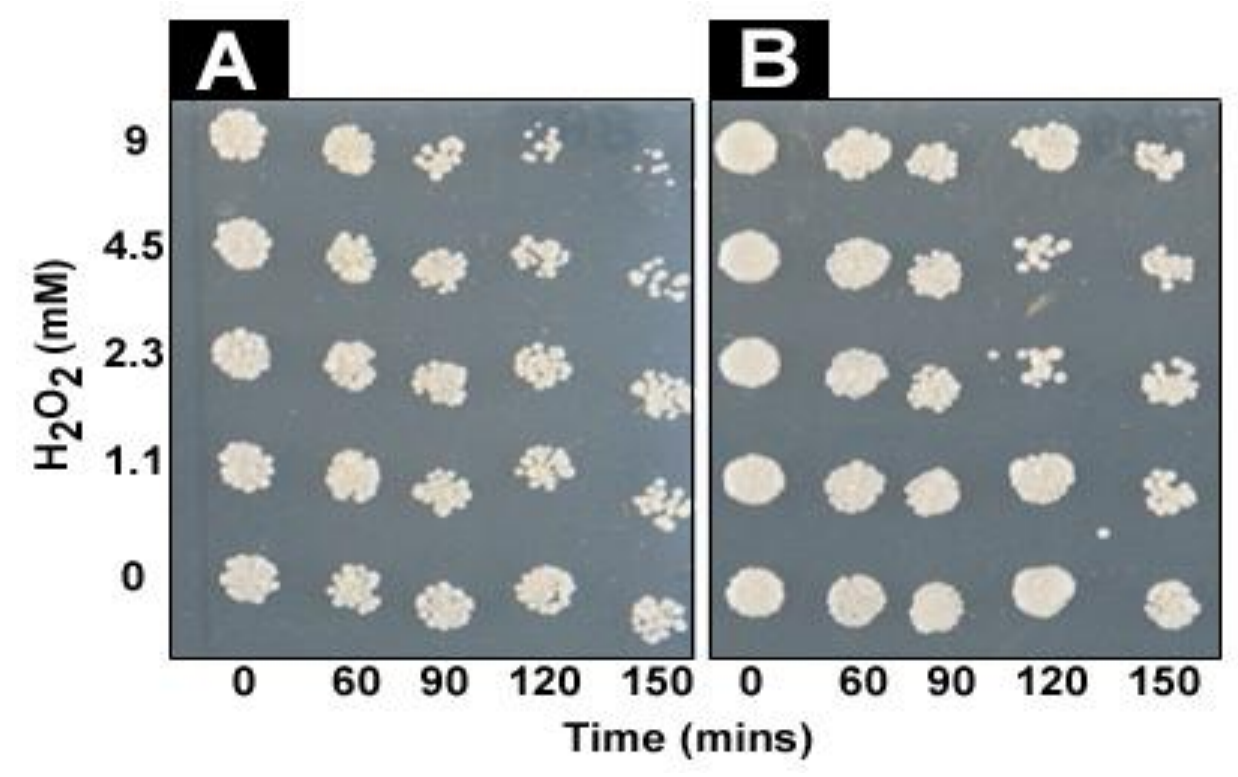

Figure 3.9. $\mathrm{TA} 8$ is more sensitive to $\mathrm{H}_{2} \mathrm{O}_{2}$ under respiratory conditions. TA8 (A) and pdrA (B) cells were treated with varying concentrations of $\mathrm{H}_{2} \mathrm{O}_{2}$ (y-axis) with glycerol-ethanol as a carbon source and incubated at $30^{\circ} \mathrm{C}$ for $2.5 \mathrm{~h}$. An aliquot of cells were spotted every 30 mins (x-axis) after an hour into $\mathrm{H}_{2} \mathrm{O}_{2}$ treatment and incubated for 2 days at $30{ }^{\circ} \mathrm{C}$. 


\subsection{Discussion}

\subsubsection{Resistant mutant generation}

The generation of resistant mutants for drug target identification has been successfully established in $S$. cerevisiae due to its genetic tractability and ease of manipulation. In many drug mode-of-action studies, resistant mutant generation has been an integral part in determining the target of a compound of interest. In this study resistant mutant generation was chosen as a method to aid in determining the modes-of-action of TA-289 and equisetin. Resistant mutants were generated by both spontaneous mutations and by treatment with a mutagen, EMS. Out of 43 resistant mutants isolated (22 TA-289-resistant and 21 equisetin-resistant mutants), only four displayed true resistance, while all other mutants displayed transient resistance to TA-289 and equisetin. The strongest resistant mutant, TA8, was chosen for further characterization. The resistance-conferring mutation in TA8 was found to be dominant. Dominant mutations suggest a gain-of-function in the protein product, such as where a cell is resistant as a result of an upregulation in multi drug resistance pumps.

Subsequently, TA8 also displayed a higher basal level of ROS compared to the parental strain $(p d r \Delta)$ used to generate the resistant mutants. Moreover, when tested against a known oxidant, $\mathrm{H}_{2} \mathrm{O}_{2}$, TA8 displayed resistance at the highest concentration tested $(9 \mathrm{mM})$. This indicated a possible mechanism by which TA8 
displays resistance to TA-289 was by upregulating its antioxidant capacities, as the mutant produces a higher basal level of ROS. The mitochondrion is a major source of ROS production. ROS are generated as a consequence of the mitochondrial respiratory chain, i.e. the ETC (electron transport chain), where these free radicals cause extensive damage to proteins, DNA, and other cellular components. For a cell that is unable to detoxify the ROS induced, prolonged exposure will ultimately lead to cell death (Sies 1985; Salmon, Evert et al. 2004). Moreover, abnormal mitochondrial function has been shown to cause an elevation of endogenous ROS (Perrone, Tan et al. 2008)(this will be further discussed in chapter 4). If the target of TA-289 and equisetin is at the mitochondria, it is possible that treatment with both compounds cause disruption to mitochondrial function, leading to elevated ROS, and cell growth inhibition. As such, a mutant resistant to TA-289 can be resistant simply by up-regulating its ability to detoxify the ROS induced by the compound. This is observed in the ROS-detection assay where the TA8 resistant mutant displays a higher level of ROS, and thus presumably, a higher level of antioxidant capacity also. This allows the mutant to become resistant to TA-289, and other oxidants, such as $\mathrm{H}_{2} \mathrm{O}_{2}$.

Additionally, the gain-of-function mutation provides an explanation to the dominant characteristics of the resistance-conferring mutation of TA8, and also possibly why the majority of the resistant mutants isolated only displayed transient 
resistance. Interestingly, when glycerol-ethanol is used as a carbon source for the cell death assays, TA8 was more sensitive to $\mathrm{H}_{2} \mathrm{O}_{2}$ treatment compared to the pdr $\Delta$ parental strain. It is possible that while TA8 produces more ROS naturally when glucose is the carbon source (i.e. under fermentative conditions), and therefore has higher antioxidant capacities. However under respiratory conditions, where ROS production is often elevated due to respiration, the resistance-conferring mutation in TA8 may prevent the normal adaptive response that would otherwise be observed in wild-type cells, wherein a threshold for upregulation of antioxidant capacities is reached in the case of the TA8 mutant.

\subsubsection{Mapping the resistance-conferring mutation}

One of the many advantages that studies using $S$. cerevisiae benefit from is the availability of tools that are available for high-throughput genome-wide analysis. The yeast genome deletion sets are exploited in a variety of ways to discover genetic and physical interactions, and for this study in particular, to probe for drug mode-ofaction. One feature of the deletion set is the addition of a unique barcode to every gene deletion, thus allowing identification by PCR amplification and hybridization to DNA microarrays. The development of DNA microarrays enables genome-wide analysis offered by the SGA methodology, but the assessment of fitness levels of thousands of genes may be performed in parallel. This method monitors whole gene expression pooled into a single tube, compared to the 14-plate array format in SGA 
analysis, thus this method requires much less drug to determine competitive fitness and functional networks. This approach takes advantage of the unique barcodes generated for each yeast ORF to analyse the barcode representation in each population. This provides a measure of relative fitness and identifies any synthetic interactions (Boone, Bussey et al. 2007).

Moreover, as the data obtained from microarray analysis can be arrayed according to chromosomal location (as they are so arrayed on plates for the SGA), it may therefore be possible to identify linkage patterns on a microarray chip. By combining the C-SGAM method as described in the introduction of this chapter, with the microarray method to analyse drug-target interactions wherein all mutants are pooled at the haploid selection step of the C-SGAM and drug-treating them in parallel (Figure 3.10), en masse, there is a significant reduction on the amount of drug needed. This is a considerable advantage, and is exemplified in the case where there is limited amount of drug to study first hand; often with natural product compounds, quantity is heavily limited. Figure 3.10 (adapted from (Boone, Bussey et al. 2007)) illustrates the main principle of the method. 


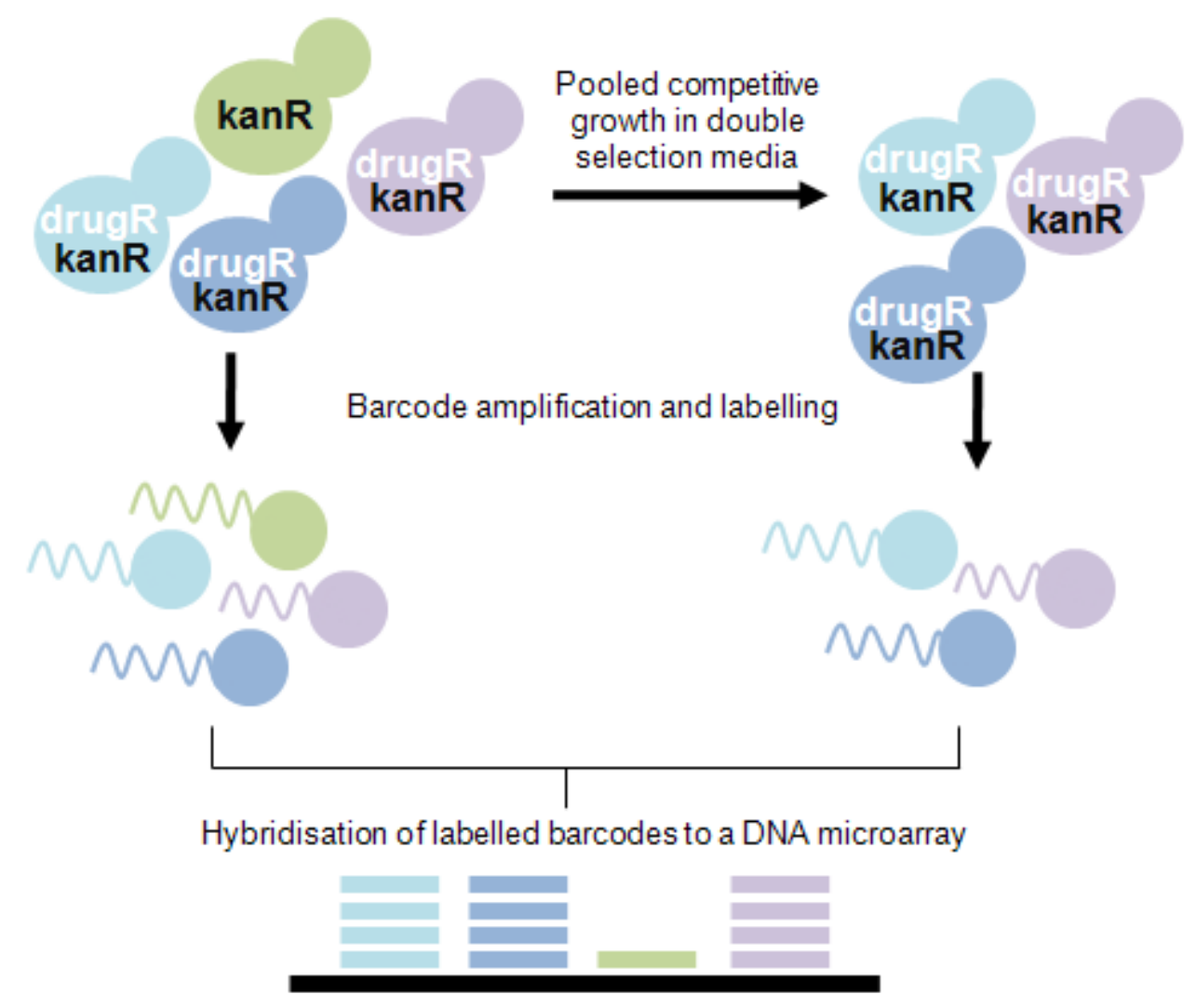

Figure 3.10. Parallel analysis by DNA microarray for resistance mapping. Populations of pooled mutant cells from the haploid $x x x \Delta$ selection in the C-SGAM methodology, each marked with unique molecular barcodes, are grown in the presence or absence of both G418 and the drug of interest (in this case, TA-289). Genomic DNA is purified, and barcodes that represent each strain are amplified by PCR using common primers labelled with fluorescent markers Cy3 (control marker) or Cy5 (drug treatment marker). Competitive hybridization of the barcode PCR products on to a microarray chip that contains corresponding oligonucleotides determines drug sensitivity of each mutant strain. This gives a quantitative read-out of the representation of each mutant in a mixed population, wherein a linkage disequilibrium pattern is identified by fluorescence intensity on the microarray chip.

Unfortunately, while the microarray method requires less drug, a major disadvantage is that by pooling of a large number of mutants and relying on fluorescence intensities, background noise within the system can result in an assay that is too insensitive to discern enough relative growth differences required to identify the mutant series comprising the linkage group. Additionally, 
methodologies used in the microarray protocol, such as genomic DNA purification and PCR amplification of the gene tags are sources of variation, all of which will undoubtedly affect the outcome of the microarray data. The loss of some data as a result of this is compensated to some extent, (to standard chemical genomic profiling screens) as most likely there will always be functionally related genes that will be identified. However, in the case where the identification of a specific pattern relies on a handful of genes located on a particular chromosome, strong signalling from the fluorescent oligonucleotide tags become increasingly important.

Unlike the pilot C-SGAM study where this method was employed to map a known resistance-conferring mutation (rpl28Q208L), a distinct linkage disequilibrium pattern was unidentifiable when mapping using the DNA microarray method (this pattern was identified in the C-SGAM on plate format, indicating that a pattern of linkage around the locus of resistance was achieved). In the microarray growth assay, the expected under-representation of neighbouring genes to $R P L 28$ was not observed (as highlighted by figure 3.6.). Nevertheless, further optimization of the microarray method may allow for a more successful approach to mapping by microarray. 
Since the C-SGAM procedure requires a substantial amount of drug to map the resistance-conferring mutation (a problem that the unsuccessful mapping by microarray method was designed to skirt-around), and as the quantity of TA-289 was limited, another approach of identifying growth conditions in lieu of sufficient quantities of drug can be employed to map the resistance-conferring mutation of the TA8 mutant. As the TA8 mutant also displayed resistance to $\mathrm{H}_{2} \mathrm{O}_{2}, \mathrm{H}_{2} \mathrm{O}_{2}$ was selected as a condition to map the resistance-conferring mutation of TA8 by the normal CSGAM method. Unfortunately, the TA8 mutant seems to either lack the ability to sporulate, or is poor at sporulating under the necessary SGA conditions. Thus the "conditions" approach for mapping of the resistance-conferring mutation of the TA8 mutant may be more successful with further optimization to aid in the sporulation step of the SGA.

Evidently, given the differences in the ability to kill or irreversibly inhibit growth in glucose and in glycerol, i.e. TA-289 and equisetin seem to kill yeast quickly in glucose, but cells do not die with treatment of these compounds in glycerol (as observed in figures 2.14 and 2.15), it is not surprising that the resistant mutant generated in this study displayed resistance most likely by preventing the triggering of cell death, rather than resistance at the actual drug target. The TA8 mutant exhibited a higher level of basal ROS compared to the parental strain, which indicates that its level of antioxidant capacity is also elevated under normal 
physiological conditions. This suggests that up-regulation of antioxidant capacity allows the TA8 mutant to prevent the induction of cell death by TA-289. A more successful approach at finding a resistance-conferring mutation may be through selecting resistant mutants in glycerol. In glucose, TA-289 and equisetin potently kill $p d r \Delta$ cells, presumably because of a combination of two factors; 1) $p d r \Delta$ cells lack an efficient efflux pump network to expel the compounds, and 2) in glucose, there is low level of respiration, and as such, low level of antioxidant capacity. However as observed in chapter two (figure 2.15), in glycerol, $p d r \Delta$ cells were more resistant, again, presumably because when cells are actively fermenting, antioxidant capacities are up-regulated, which are enough to counteract the effects of TA-289 and equisetin even when they are efflux pump-compromised. This suggests that respiring cells are more resistant to killing by TA-289 and equisetin due to the upregulation of antioxidant capacity, and are able to adapt to the stress caused by these compounds. Additionally, in the case where TA-289 and equisetin do target the mitochondria, the target(s) will become more essential under respiratory conditions, and it is assumed that mutation in the target(s) will provide strongest resistance to the compounds. Therefore, under respiratory conditions, it is less likely that a mutant conferring resistance to the compounds will be resistant simply by preventing apoptosis (as observed in the TA8 mutant isolated in this chapter), and as such the mutation may be more associated with the mechanism of TA-289 and equisetin, and upon further optimization of microarray techniques, the resistanceconferring mutation may be mapped. 


\section{REACTIVE OXYGEN SPECIES IN Saccharomyces cerevisiae}

\subsection{The role of mitochondria in the production of reactive oxygen species}

Reactive oxygen species (ROS) are reactive agents produced as a result of the respiratory and metabolic processes in all aerobically growing organisms and microorganisms. These reactive agents include free radicals and peroxides such as superoxide anions $\left(\cdot \mathrm{O}^{2}-\right)$, hydroxyl radicals $(\cdot \mathrm{OH})$, hydrogen peroxide $\left(\mathrm{H}_{2} \mathrm{O}_{2}\right)$, alkoxy (RO.) and peroxy (ROO.) radicals, the accumulation of which results in oxidative stress (Sies 1985; Rice-Evans 1995). Oxidative damage caused by intracellular ROS leads to significant deleterious damage to cellular components such as proteins, lipids, and DNA (Salmon, Evert et al. 2004).

Mitochondria are a major source of endogenous ROS, the production of which stems from leakage of electrons from within the mitochondrial respiratory electron transport chain (ETC), resulting in the generation of ROS. The ETC comprises four complexes (complex I-IV) and F0-F1 ATP-synthase (sometimes referred to as complex V) present in the inner mitochondrial membrane (IMM). Complexes I-IV transfer electrons from donors (such as NADH (reduced nicotinamide adenine dinucleotide)(complex I; NADH dehydrogenase) and succinate (complex II; succinate dehydrogenase)) to the terminal acceptor oxygen $\left(\mathrm{O}_{2}\right)$, and the protons $\left(\mathrm{H}^{+}\right)$released into the intermembrane space by complexes I, III (bc1 complex, 
cytochrome $c$ reductases), and IV (cytochrome $c$ oxidase), are used by ATP-synthase to convert ADP (adenosine diphosphate) to ATP (adenosine triphosphate). This charge distribution is known as the mitochondrial transmembrane potential $\left(\Delta \Psi_{\mathrm{m}}\right)$, and is required for the synthesis of ATP (Ricci, Waterhouse et al. 2003) (Figure 4.1).

\section{Intermembrane}

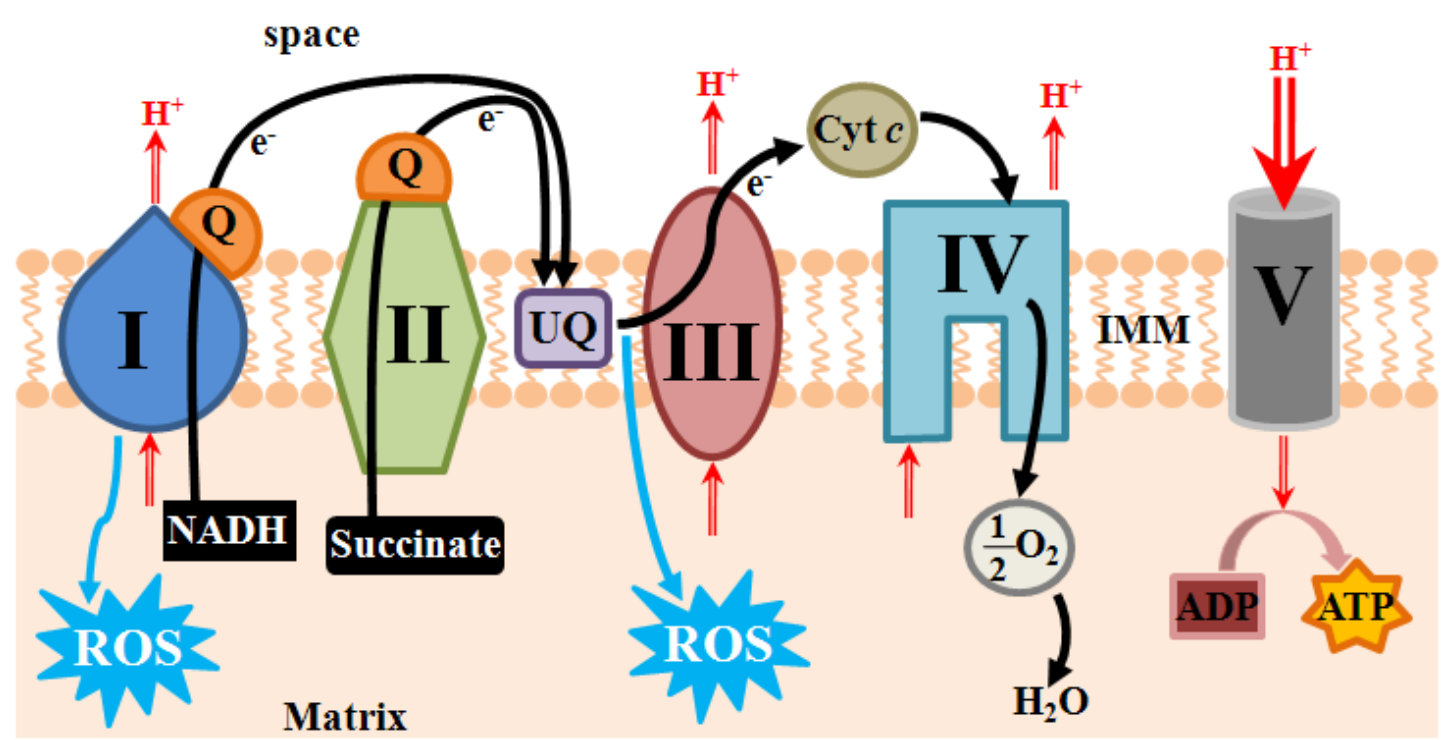

Figure 4.1. A simplified model of the mitochondrial ETC and ROS production. Electrons (e-) enter the ETC via complex I and II. Complex I uses the conversion of NADH to NAD ${ }^{+}$, while complex II, the conversion of succinate to fumarate to transfer e- from coenzyme Q (Q) to ubiquinone (UQ), which then passes e- to complex III. Cytochrome $C$ (Cyt $c$ ) transfers e- from complex III ( $b c 1$ complex, cytochrome $c$ reductase) to complex IV (cytochrome $c$ oxidase), which on acceptance of the e-, converts $\mathrm{H}^{+}$and $\mathrm{O}_{2}$ into $\mathrm{H}_{2} \mathrm{O}$. All complexes except for complex II pump protons $\left(\mathrm{H}^{+}\right)$into the intermembrane space. This charge distribution $\left(\Delta \Psi_{\mathrm{m}}\right.$, the mitochondrial transmembrane potential) is used by ATP-synthase (complex V) to convert ADP into ATP. ROS is mainly produced by complexes I and III during this process (Ricci, Waterhouse et al. 2003).

Complexes in the ETC thought to be responsible for most of the ROS production include the redox-cycling ubiquinone complex III, and complex I, where hyperpolarization of the mitochondrial transmembrane potential $\left(\Delta \Psi_{\mathrm{m}}\right)$ results in 
reverse electron transfer (Turrens, Alexandre et al. 1985; Kushnareva, Murphy et al. 2002; Ricci, Waterhouse et al. 2003), and complex II (succinate dehydrogenase) (Zhang, Yu et al. 1998) has also been previously shown to be a source of ROS.

In the event of premature electron leakage to oxygen, the ROS superoxide anion $\cdot \mathrm{O}_{2}-$, in particular is generated. The detoxification of superoxide by the antioxidant enzymes superoxide disumutases (Gralla, Kosman et al. 1992) produces $\mathrm{H}_{2} \mathrm{O}_{2}$, which can readily cross most biological membranes. The most deleterious effects arise when $\mathrm{H}_{2} \mathrm{O}_{2}$ is converted to the highly reactive hydroxyl radical, which in contrast to the superoxide anion reacts indiscriminately with most macromolecules within the cell, often producing more free radicals in the process (Perrone, Tan et al. 2008). Hydroxyl radicals are generated through the catalysis of superoxides and $\mathrm{H}_{2} \mathrm{O}_{2}$ by transition metal ions such as $\mathrm{Fe}^{2+}$ (via the Fenton-Haber Weiss reaction (Haber and Weiss 1932; Koppenol 2001)). This process is cyclic, reinitiated each time by the superoxide anion (Halliwell 1995; Temple, Perrone et al. 2005; Perrone, Tan et al. 2008). For a cell that is unable to maintain its redox state and ion homeostasis, this detrimental event will eventually lead to cell death (Madeo, Carmona-Gutierrez et al. 2009; Tan, Teo et al. 2009).

There are a few key factors that affect mitochondrial ROS production and oxidative damage to the cell as a result. It has been well established in a number of eukaryotic 
organisms that mitochondrial respiratory capacity and mitochondrial inner membrane potential play significant roles in the degree of ROS generation (Perrone, Tan et al. 2008). Hyperpolarization of the mitochondrial inner membrane leads to disruption of the mitochondrial transmembrane potential $\left(\Delta \Psi_{\mathrm{m}}\right)$, and as a result of this, increased ROS production (Machida and Tanaka 1999). Mutations and/or small molecule inhibitors that impair the ETC in $S$. cerevisiae have been associated with elevated ROS production, of which the magnitude of ROS production is dependent on the nature of the disruption to the mitochondrial respiratory capacity (Barros, Netto et al. 2003).

\subsection{Mitochondrial ROS production and yeast apoptosis}

Mitochondria-mediated cell death often arises through loss of mitochondrial function; collapse of the $\Delta \Psi_{\mathrm{m}}$, mitochondrial fragmentation and/or swelling, and release of cytochrome $c$ from the inner mitochondrial membrane (IMM) to the cytosol. This is the case for drug-induced cell death by amiodarone, where the compound increases cytostolic $\mathrm{Ca}^{2+}$, causing hyperpolarization of the $\Delta \Psi_{\mathrm{m}}$, and induction of ROS from complex III of the ETC leading to an eventual collapse of the $\Delta \Psi_{\mathrm{m}}$, and cytochrome $c$ release (Pozniakovsky, Knorre et al. 2005). Alternatively, cell death can occur through the translocation of mitochondrial endonucleases such as apoptosis-inducing factor $A I F 1$ to the nucleus, where it mediates chromatin condensation and DNA degradation (Madeo, Carmona-Gutierrez et al. 2009). Exposure to oxidant $\mathrm{H}_{2} \mathrm{O}_{2}$ causes ROS generation, leading to the induction of AIF1 
in programmed cell death (Madeo, Frohlich et al. 1999). In both cases, the induction of ROS has been implicated as an important up-stream component in mediating programmed cell death. In the case where cell death occurs through mitochondrialmediated apoptosis, ROS generation is almost always present. However, the mechanisms by which ROS generation mediate the triggering of apoptotic effectors is not fully understood, and additionally, the challenge also remains in defining the exact site of ROS production (Perrone, Tan et al. 2008).

\subsection{Inhibitors of the electron transport chain and the generation of reactive oxygen species}

Many biologically active compounds that cause cell death in yeast do so via mitochondrial pathways, including ETC inhibitors such as the complex I-inhibitor rotenone (Ramsay, Krueger et al. 1991), the complex II-inhibitors atpenins (Miyadera, Shiomi et al. 2003), the complex III-inhibitors antimycin A (Chen, Vazquez et al. 2003; Lai, Zhang et al. 2005) and myxothiazol (Thierbach and Reichenbach 1981; Thierbach and Michaelis 1982), the complex IV-inhibitor potassium cyanide (KCN) (Delhumeau, Cruzmendoza et al. 1994), and the complex V-inhibitor oligomycin (Bertina, Steenstra et al. 1974). Paraquat is a compound that acts as an alternative terminal acceptor at complex IV, becoming a free radical itself and causing damage in that way (Cocheme and Murphy 2008) (figure 4.2).

Notably, while it may be presumed that inhibition of any one complex causes disruption to its function and thus ROS production at that site, it is not necessarily 
the case. Complex I inhibitor rotenone for example, causes ROS production proximal to the rotenone binding site, thought to be located between flavin mononucleotide (a co-chaperone of NADH dehydrogenase (Rao, Mackler et al. 1963)) and the binding site of rotenone. Additionally, ROS generation caused by certain ETC inhibitors can be alleviated by a compound that inhibits the ETC upstream of the binding site, as it was in the case of edelfosine (Zhang, Gajate et al. 2007), and farnesol (Machida, Tanaka et al. 1998), whose inhibitory effects leading to the generation of ROS were perturbed by rotenone. Furthermore, inhibition at any complex of the ETC does not always result in stimulation of ROS production, namely superoxide anions, by the target complex. ROS accumulation and/or production are dependent upon the site at which the inhibitor targets. Take for example complex III inhibitors antimycin A, and myxothiazol. The production of unstable semiquinones at complex III during oxidation of coenzyme $Q$ quinol molecules has been suggested to be the major source of superoxide anion production at this complex (figure 4.2). 


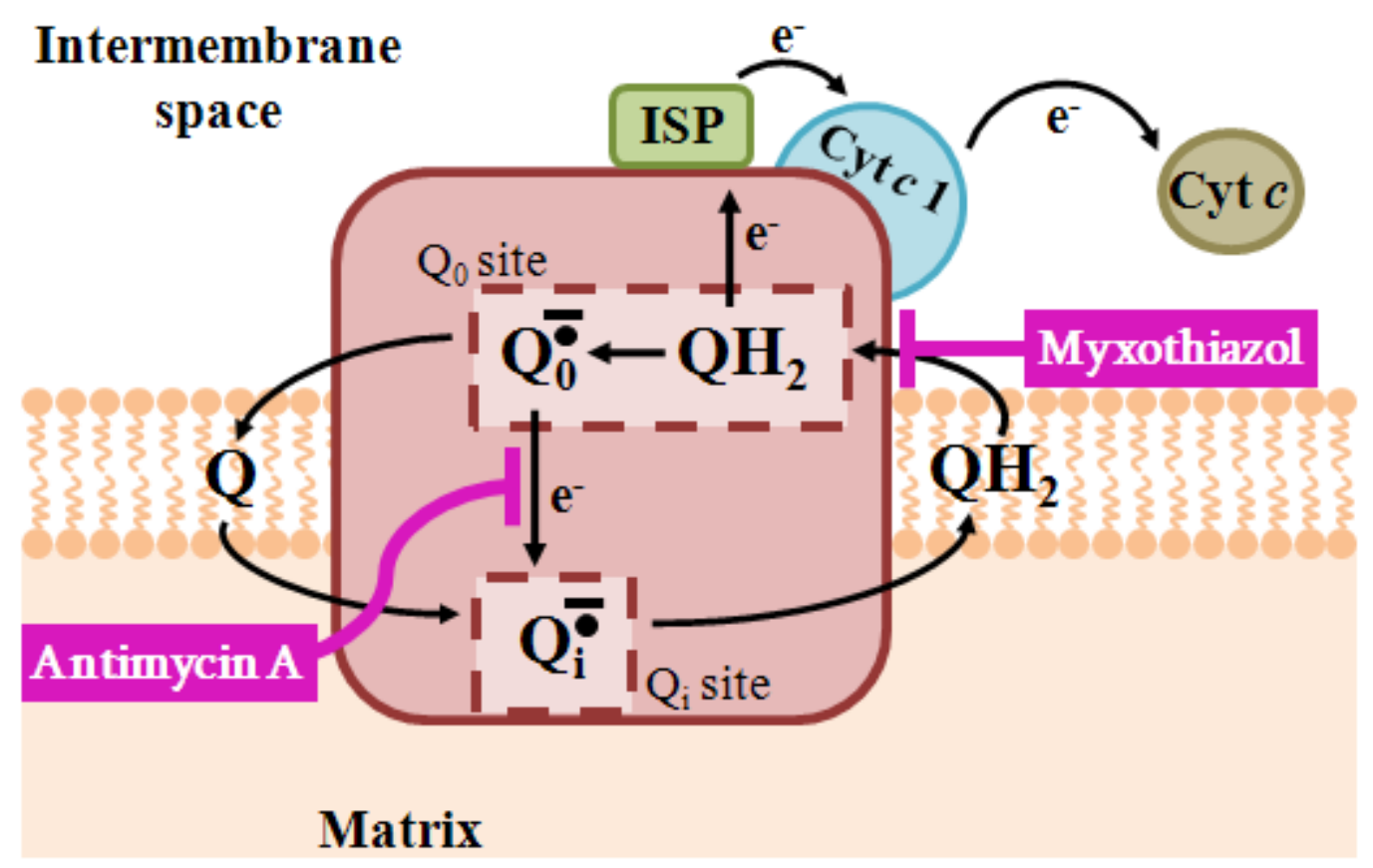

Figure 4.2. A simplified model of the Q-cycle at complex III and the mechanism of superoxide production. Complex III oxidises coenzyme $\mathrm{Q}$ quinol $\left(\mathrm{QH}_{2}\right)$, the cycle of which is known as the Q-cycle. The first step involves oxidation of $\mathrm{QH}_{2}$ at the $\mathrm{Q}_{0}$ site, in which an electron (e-) is transferred to the iron-sulfur protein (ISP), cytochrome $c 1$ (Cyt $c 1$ ), and cytochrome $c$ (Cyt $c$ ), which then passes it along to the next complex in the ETC, cytochrome c oxidase (complex IV, not shown in this figure). This oxidation of QH2 to transfer an e- down the ETC creates an unstable semiquinone ( $\left.\mathrm{Q}_{0}{ }^{-}\right)$, which donates an e- through conducting pathways from the $\mathrm{Q}_{0}$ site to the $\mathrm{Q}_{\mathrm{i}}$ site. At the $\mathrm{Qi}$ site, a coenzyme $\mathrm{Q}$ molecule $(\mathrm{Q})$ is reduced by the donated e- into $\mathrm{QH}_{2}$, and the cycle begins again. The sites of inhibition by antimycin A and myxothiazol are as illustrated (Andreyev 2005).

Antimycin A inhibits the electron transfer of the unstable semiquinone to the site where it is reduced to a quinol molecule, causing an accumulation of unstable semiquinone molecules and thus promoting superoxide anion production (Lai, Zhang et al. 2005). On the other hand, myxothiazol acts at the Qo site of complex III, preventing formation of the unstable semiquinone, and this inhibition at complex III of the ETC has indeed been shown to reduce superoxide production (Thierbach and Reichenbach 1981; Turrens, Alexandre et al. 1985; Korshunov, Skulachev et al. 
1997). Notably, while myxothiazol inhibits superoxide production by complex III, it has been shown to stimulate $\mathrm{H}_{2} \mathrm{O}_{2}$ production at a site distal to its binding site in complex III (Starkov and Fiskum 2001). Hence, ROS production can still occur even when an ETC inhibitor such as myxothiazol prevents superoxide production. In the cases where ROS is produced, the challenge remains in determining the site of ROS production; as described previously, ROS production can occur up-stream or downstream of the binding site, and is also dependent on the type on inhibition. 


\subsection{Aims and objectives}

The aim of this chapter was to determine whether TA-289 and equisetin cause cell death by ROS production in yeast, and whether the production originates from within the mitochondria. This will include:

- In vivo detection of ROS

- Assessment of mitochondrial activity and morphology

Part of this study involved testing a select number of gene deletions hypothesised to be potential targets of TA-289 and equisetin. 


\subsection{Materials and methods}

\subsubsection{Yeast strains}

All $S$. cerevisiae strains used in this study are as follows: * this mutant and the 41

following = mutants used in this study from the yeast gene deletion set.

\begin{tabular}{|c|c|}
\hline Strain & Genotype \\
\hline YCG100 (BY4741) & 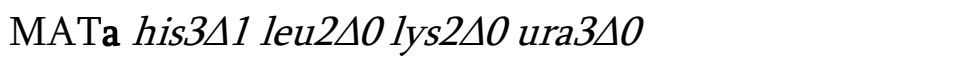 \\
\hline YCG289 (yeGFP) & 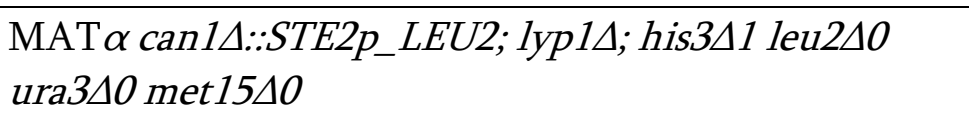 \\
\hline YCG326 (pdr $\Delta)$ & 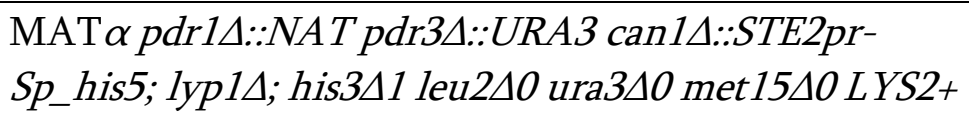 \\
\hline YCG387 & 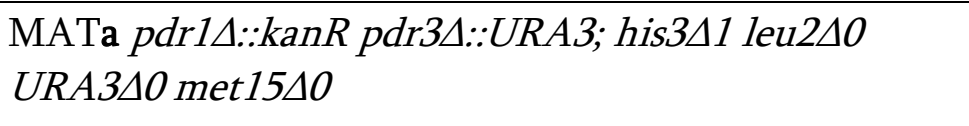 \\
\hline $\begin{array}{l}\text { YOR356W (Cir2p- } \\
\text { GFP) }\end{array}$ & Derived from BY4741 \\
\hline *aac14 & Derived from BY4741; aac1 $\because: k a n R$ \\
\hline aac34 & Derived from BY4741; aac34::kanR \\
\hline atp1D & Derived from BY4741; atp14::kanR \\
\hline $\operatorname{atp} 5 \Delta$ & Derived from BY4741; atp54::kanR \\
\hline acold & Derived from BY4741; aco1 $\triangle:: k a n R$ \\
\hline aco2 2 & Derived from BY4741; aco2 $\triangle:: k a n R$ \\
\hline aif1 & Derived from BY4741; aif1 $:: k a n R$ \\
\hline $\operatorname{ctt} 1 \Delta$ & Derived from BY4741; ctt1 $4:: k a n R$ \\
\hline $\operatorname{dic} 1 \Delta$ & Derived from BY4741; dic1 $\because: k a n R$ \\
\hline $\operatorname{mir1\Delta }$ & Derived from BY4741; mir14::kanR \\
\hline odc1 $1 \Delta$ & Derived from BY4741; odc1 $:: k a n R$ \\
\hline
\end{tabular}




\begin{tabular}{|c|c|}
\hline odc2 $2 \Delta$ & Derived from BY4741; odc24::kanR \\
\hline pic24 & Derived from BY4741; pic24::kanR \\
\hline$s f_{c} 1 \Delta$ & Derived from BY4741; sfc1 $::$ kanR \\
\hline por24 & Derived from BY4741; por $24:: k a n R$ \\
\hline mcald & Derived from BY4741; mca14::kanR \\
\hline rad94 & Derived from BY4741; rad94::kanR \\
\hline $\operatorname{sod} 1 \Delta$ & Derived from BY4741; sod14::kanR \\
\hline $\operatorname{sod} 2 \Delta$ & Derived from BY4741; sod24::kanR \\
\hline ura1A & Derived from BY4741; ura14::kanR \\
\hline gpd1A & Derived from BY4741; gpd14::kanR \\
\hline$g p d 2 \Delta$ & Derived from BY4741; gpd2ム::kanR \\
\hline gut2A & Derived from BY4741; gut2L::kanR \\
\hline $\operatorname{coq} 1 \Delta$ & Derived from BY4741; coq14::kanR \\
\hline $\operatorname{coq} 2 \Delta$ & Derived from BY4741; coq24::kanR \\
\hline $\operatorname{cor} 1 \Delta$ & Derived from BY4741; cor14::kanR \\
\hline $\operatorname{cox} 5 A \Delta$ & Derived from BY4741; cox5A4::kanR \\
\hline $\operatorname{cox} 64$ & Derived from BY4741; cox64::kanR \\
\hline $\operatorname{cox} 7 \Delta$ & Derived from BY4741; cox74::kanR \\
\hline $\operatorname{cox} 94$ & Derived from BY4741; cox94::kanR \\
\hline cyt1A & Derived from BY4741; cyt14::kanR \\
\hline pet1224 & Derived from BY4741; pet1224::kanR \\
\hline pet $494 \Delta$ & Derived from BY4741; pet4944::kanR \\
\hline nde1A & Derived from BY4741; nde14::kanR \\
\hline$q c r 2 \Delta$ & Derived from BY4741; qcr24::kanR \\
\hline qcr64 & Derived from BY4741; qcr64::kanR \\
\hline$q c r 7 \Delta$ & Derived from BY4741; qcr74::kanR \\
\hline
\end{tabular}




\begin{tabular}{|c|c|}
\hline qcr84 & Derived from BY4741; qcr84::kanR \\
\hline rip1s & Derived from BY4741; rip14::kanR \\
\hline sdh1A & Derived from BY4741; sdh14::kanR \\
\hline sdh24 & Derived from BY4741; sdh24::kanR \\
\hline emi54 & Derived from BY4741; emi54::kanR \\
\hline
\end{tabular}

\subsubsection{Growth media}

All yeast cultures were performed in one of the following media as prescribed previously, supplemented with either G418 (200 $\mu \mathrm{g} / \mathrm{mL}$ final concentration) or NAT (100 $\mu \mathrm{g} / \mathrm{mL}$ final concentration), unless otherwise stated.

\subsubsection{Compounds and chemicals}

Purchased from Invitrogen NZ Ltd (Auckland, NZ): MitoTracker ${ }^{\circledR}$ Green FM at a working stock of $20 \mathrm{mM}$ prepared in DMSO and stored in the dark at $-20{ }^{\circ} \mathrm{C}$.

Purchased from Sigma-Aldrich Life Science and Biochemicals: Propidium iodide at a working stock concentration of $10 \mathrm{mg} / \mathrm{mL}$ prepared in $\mathrm{ddH}_{2} \mathrm{O}$ and stored in the dark at $4{ }^{\circ} \mathrm{C}, 2,7$-dichlorofluorescein diacetate (DCF) at a working stock of $1 \mathrm{mM}$ prepared in DMSO and stored in the dark at $-20{ }^{\circ} \mathrm{C}$, rapamycin at a working stock concentration of $0.4 \mathrm{mg} / \mathrm{mL}$ prepared in DMSO and stored at $-20{ }^{\circ} \mathrm{C}$, hydrogen peroxide $\left(\mathrm{H}_{2} \mathrm{O}_{2}\right)$ at a working stock concentration of $0.9 \mathrm{M}$ prepared in $\mathrm{ddH}_{2} \mathrm{O}$ and stored in the dark at $4{ }^{\circ} \mathrm{C}$. 


\subsubsection{Detecting reactive oxygen species by flow cytometry}

Cell concentrations of overnight cultures of $p d r \Delta$ were determined by measuring OD at $600 \mathrm{~nm}$, and subsequently diluted to $1 \times 10^{7}$ cells $/ \mathrm{mL}$ in fresh SC media. $p d r \Delta$ cells were treated with either TA-289 or equisetin (100 $\mu \mathrm{M}$ final concentration) and incubated for either 30 mins or 60 mins at $30{ }^{\circ} \mathrm{C} . \mathrm{H}_{2} \mathrm{O}_{2}$ (1mM final concentration) served as a positive control. The procedure for DCF-staining and visualization were carried out as per chapter three method 3.4.11.

\subsubsection{Detecting mitochondrial activity by flow cytometry}

Briefly, cell concentrations of overnight cultures of wt and $p d r \Delta$ were determined by measuring $\mathrm{OD}$ at $600 \mathrm{~nm}$ and subsequently diluting in SC to given final cell concentrations of $1 \times 10^{7}$ cells $/ \mathrm{mL}$, and divided into $100 \mu \mathrm{L}$ aliquots in sterile microcentrifuge tubes. Cells were treated with either $25 \mu \mathrm{M}$ or $50 \mu \mathrm{M}$ TA-289 and equisetin, mixed by brief vortexing, and incubated at $30{ }^{\circ} \mathrm{C}$ for $1 \mathrm{~h}$. DMSO (1\% final concentration) served as a carrier control. After 15 mins into treatment, cells were stained with MitoTracker ${ }^{\circledR}$ Green FM (200 nm final concentration) and then further incubated. Cells were then collected by centrifugation, washed, and resuspended in $100 \mu \mathrm{L}$ SC.

For flow cytometry analysis of mitochondrial staining by MitoTracker of wt and $p d r \Delta$, cells were diluted with $400 \mu \mathrm{L} \mathrm{ddH}_{2} \mathrm{O}$, kept on ice and co-stained with 6 $\mu \mathrm{g} / \mathrm{mL}$ propidium iodide prior to visualizing. Flow cytometry data obtained was 
analysed on FlowJo version 7.6.1 flow cytometry analysis software (Tree Star Inc., Ashland, OR 97520).

\subsubsection{High-throughput visualization of mitochondria}

Briefly, an overnight culture of YOR356W (a Cir2p-GFP fusion protein expressing strain; Cir2p-GFP has been previously shown to localize in the mitochondria and is highly expressed (Huh, Falvo et al. 2003).) was diluted to $1 \times 10^{7}$ cells/mL in either fresh SC media, or SC-gly-EtOH, from which $100 \mu \mathrm{L}$ aliquots were transferred into sterile microcentrifuge tubes. Cells were then treated with either TA-289 (50 $\mu \mathrm{M}$ final concentration), or equisetin $(50 \mu \mathrm{M}$ final concentration). DMSO (1\% final concentration) served as a carrier control. The cells were mixed by brief vortexing, and then incubated for $1 \mathrm{~h}$ at $30{ }^{\circ} \mathrm{C}$. Photographs of the treated and untreated cells were taken 30 mins into the incubation, and again at the end of the incubation. In preparation for visualization of mitochondria morphology, $50 \mu \mathrm{L}$ aliquots of treated and untreated cells were dispensed into microplates and visualized using the Opera ${ }^{\circledR}$ confocal microplate imaging reader (PerkinElmer Inc, CA, USA).

\subsubsection{Liquid dose-response assays}

All liquid dose-response assays were prepared as per methods previously described in chapter two (method 2.4.5) for dose-response of BY4742 (wt) and pdr $\Delta$ cells to TA-289 and equisetin. 
For dose-response assays conducted using glucose as a carbon source, see chapter two (method 2.4.5). The plate was incubated for three days at $30^{\circ} \mathrm{C}$, and cell density was measured on day three.

For dose-response assays conducted using glycerol-ethanol as a carbon source, glycerol ( $2 \%$ final concentration) and ethanol ( $2 \%$ final concentration) were added to fresh SC media instead of glucose. In addition, $100 \mu \mathrm{L}$ of ddH2O was added to all other wells that did not contain experimental samples, to prevent the experimental wells from drying out. The plate was kept in a plastic sleeve and incubated for six days at $30^{\circ} \mathrm{C}$, taken out each day and mixed vigorously before placing back into the incubator. Optical density was measured (590 nm) on day six.

For dose-response assays in the presence of $0.7 \mathrm{M} \mathrm{NaCl}$, wells containing $\mathrm{NaCl}$ with no drug served as a solvent control. Plates were incubated at $30{ }^{\circ} \mathrm{C}$ for $24 \mathrm{~h}$ and cell density measured as per normal.

\subsubsection{Testing candidate gene targets}

A selection of deletion strains were grown overnight at $30^{\circ} \mathrm{C}$ in YPD media in a $96-$

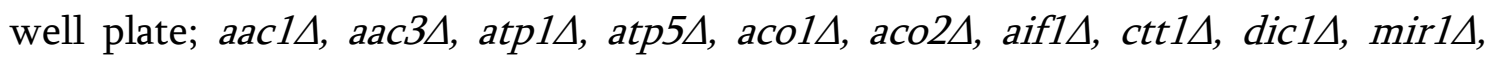

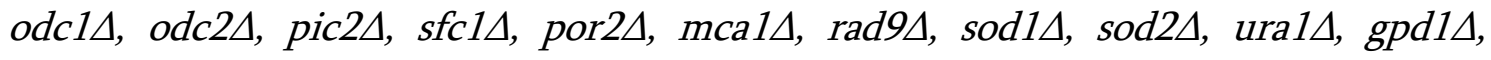

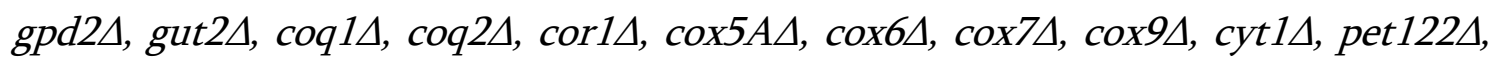

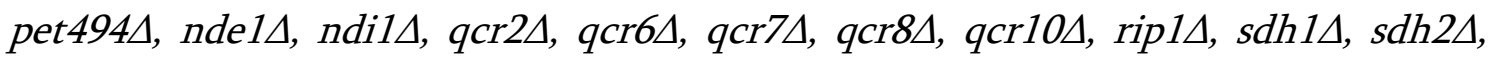


and emi5 4 . Cell concentration of each strain was determined by measuring OD at $600 \mathrm{~nm}$, and subsequently diluted to $1 \times 10^{7}$ cells/mL in fresh SC media.

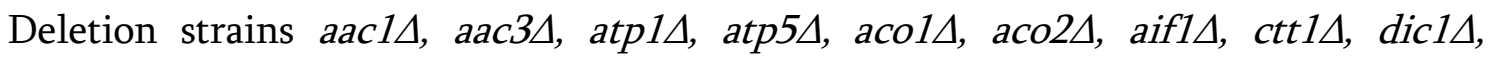

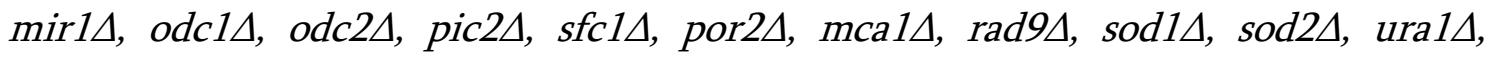
gpd14, gpd2A, and gut2A, and wt (BY4741) were treated with $50 \mu \mathrm{M}$ TA-289 for 18 h at $30{ }^{\circ} \mathrm{C}$, after which cell density (OD $590 \mathrm{~nm}$ ) was measured. Residual growth relative to wt was plotted and resistance/sensitivity of each strain was determined at $1 \%$ significance.

Deletion strains coq14, coq2A, cor14, $\operatorname{cox} 5 A \Delta, \operatorname{cox} 64, \operatorname{cox} 7 \Delta, \operatorname{cox} 9 \Delta, \operatorname{cyt} 1 \Delta$, pet $122 \Delta$,

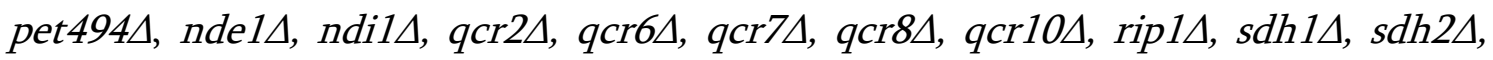
and emi5 , and BY4741 were treated with $15 \mu \mathrm{M}, 30 \mu \mathrm{M}$, and $60 \mu \mathrm{M}$ equisetin for $18 \mathrm{~h}$ at $30^{\circ} \mathrm{C}$, after which cell density (OD $590 \mathrm{~nm}$ ) was measured. Residual growth relative to wt was plotted and resistance/sensitivity of each strain was determined at $1 \%$ significance. 


\section{$\underline{4.4 \text { Results }}$}

\subsubsection{TA-289 causes the production of reactive oxygen species}

Based on the mitochondrial hits attained from the chemical genomic profiling of TA-289 (chapter two, result 2.5.5), and as the resistant mutant generated in chapter three (result 3.6.4) displayed resistance by up-regulating antioxidant capacity, both TA-289 and equisetin were tested for their ability to increase the production of reactive oxygen species (ROS) in yeast. The oxidant sensitive fluorescent stain, DCF, was used to measure ROS production within cells. When DCF crosses the cell membrane, it is hydrolysed, and in the presence of ROS, oxidised to the highly fluorescent dichlorofluorescein (Wang and Joseph 1999). In this study, propidium iodide was also used as a counter stain to detect permeablized cells (which are presumably dead). The $p d r \Delta$ cells treated with TA-289 displayed a large increase in ROS production compared not only to the untreated control, but also to the positive control, $\mathrm{H}_{2} \mathrm{O}_{2}$, within 30 mins of treatment (Figure 4.3). 


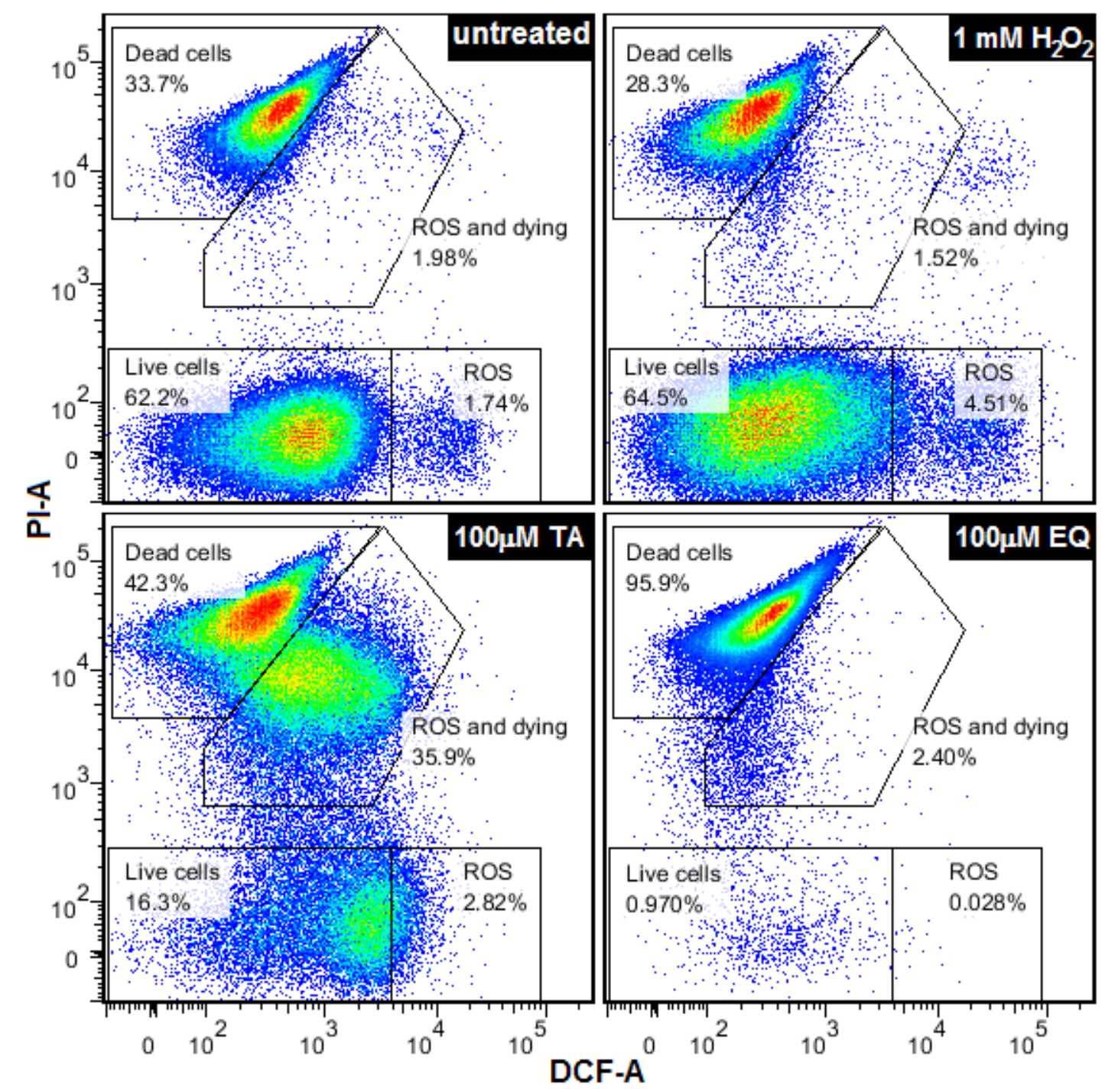

Figure 4.3. TA-289 causes production of ROS. pdrA cells were treated with TA-289 and equisetin, and stained with DCF ( $\mathrm{x}$-axis) to detect ROS and propidium iodide (y-axis) to detect dead cells. Cell populations were gated according to the untreated control.

A large population of TA-289-treated cells were also positively stained for propidium iodide, suggesting that prolonged ROS production eventually leads to cell death; propidium iodide stains cells with damaged membranes, where cell wall permeability is an important characteristic for viable cells (Jones and Senft 1985). After 60 mins of TA-289 treatment, the population of cells producing ROS 
decreased, while the percentage of propidium iodide-permeable cells increased, further indicating that cell wall permeablization occurs as a result of ROS production (Table 4.1). Almost all the cells treated with equisetin were propidium iodide-permeable within 30 mins, with only a very small population producing ROS. These observations indicate that TA-289 may cause cell death by inducing the production of ROS, and that both compounds display fast kinetics in that respect this is especially so in the case for equisetin, mirroring the kinetics observed in the cell death assays (chapter two, result 2.5.6) (Figure 4.3, Table 4.1).

\begin{tabular}{|l|c|c|c|c|}
\hline $\begin{array}{r}\text { \% Cell } \\
\text { population }\end{array}$ & Live & ROS & $\begin{array}{c}\text { ROS and } \\
\text { dying }\end{array}$ & Dead \\
\hline Untreated & 62.20 & 1.74 & 1.98 & 33.70 \\
\hline $100 \mu \mathrm{M}$ TA-289, t30 & 16.30 & 2.82 & 35.90 & 42.30 \\
\hline $100 \mu \mathrm{M}$ TA-289, t60 & 5.18 & 0.18 & 19.70 & 73.10 \\
\hline $100 \mu \mathrm{M}$ EQ, t30 & 0.97 & 0.03 & 2.40 & 95.90 \\
\hline $100 \mu \mathrm{M}$ EQ, t60 & 0.39 & 0.06 & 0.90 & 98.10 \\
\hline
\end{tabular}

Table 4.1. Table showing percentage of live, ROS, ROS and dying, and propidium iodidepermeable cell populations. Untreated samples were compared to cells treated for 30 mins (t30) and 60 mins (t60) with TA-289 and equisetin.

\subsubsection{TA-289 and equisetin effect mitochondria and alter mitochondrial morphology} Altered ROS production is often indicative of perturbed mitochondrial activity (Andreyev 2005; Sedensky and G. Morgan 2006). Cells treated with TA-289 and equisetin were stained with a fluorescent stain specific for mitochondria, and morphological changes assessed by flow cytometry. A leftward shift in fluorescence intensity of the mitochondrial stain, MitoTracker ${ }^{\circledR}$ Green FM, in wt cells treated 
with TA-289 indicates a disruption in mitochondrial uptake of the stain, an effect that is more pronounced in $p d r \Delta$ cells. In cells treated with equisetin, stain uptake is significantly disrupted, with lower cell counts indicating a higher population of cell death in equisetin-treated cells compared to the less potent TA-289 (Figure 4.4).

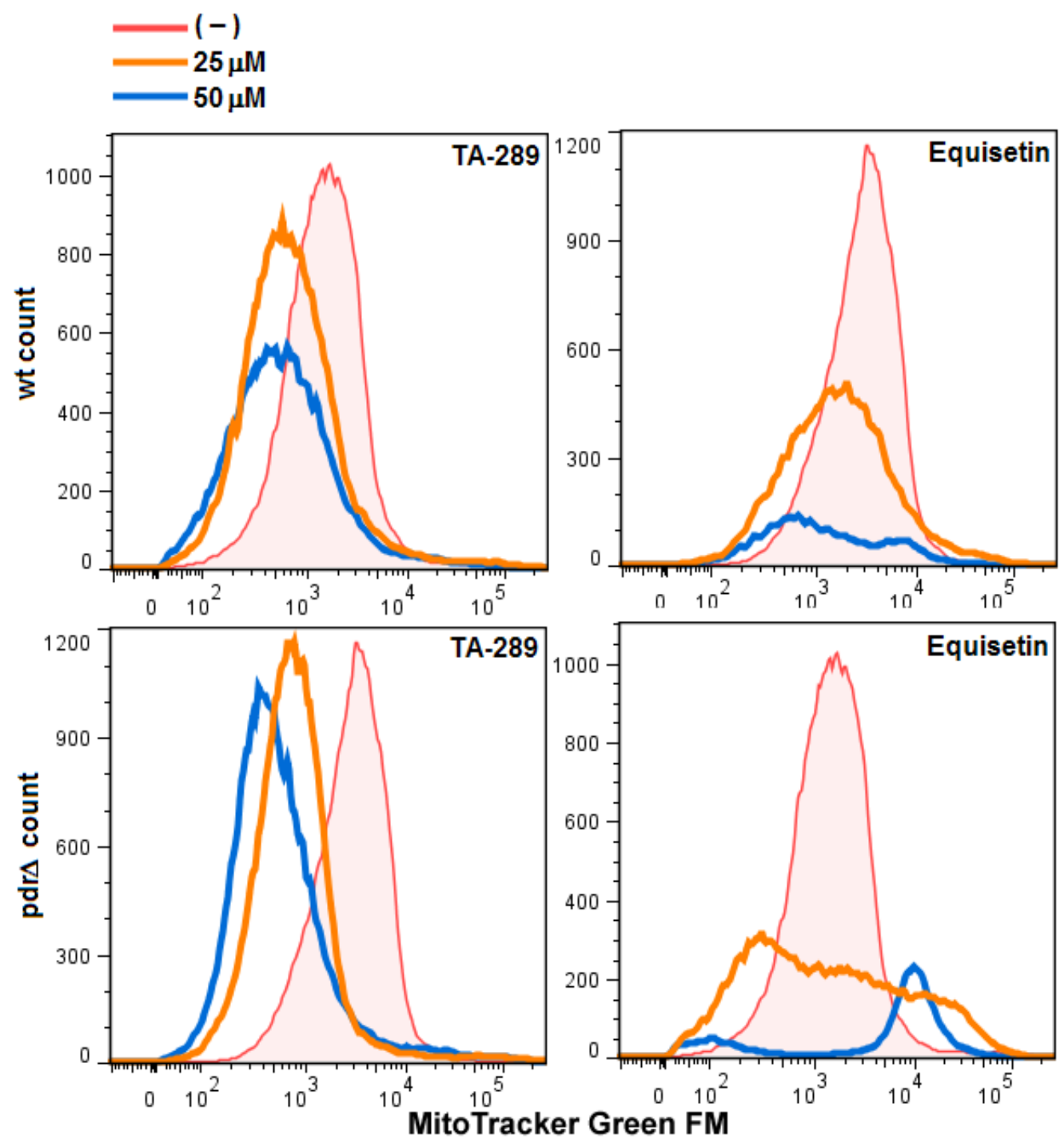

Figure 4.4. TA-289 and equisetin have an affect on mitochondria. wt (Top) and $\operatorname{pdr} \Delta$ (Bottom) cells treated with $25 \mu \mathrm{M}$ (orange line) and $50 \mu \mathrm{M}$ (blue line) TA-289 and equisetin were stained with MitoTracker ${ }^{\odot}$ Green FM and visualized. A shift in fluorescence intensity and a decrease in treated cell populations compared to the untreated controls (pink line) indicate that TA-289 and equisetin have an effect on the mitochondria. 
Mitochondrial morphology was also assessed by confocal microscopy using a strain expressing a Cir2p-GFP fusion protein, which localizes to the mitochondria. Mitochondrial morphology was severely altered in equisetin-treated cells (Figure 4.5.C) after 30 mins treatment compared to untreated controls (Figure 4.5.A). There was some morphological changes observed in TA-289-treated cells (Figure 4.5.B), though only slightly compared to that caused by equisetin. The slight peak observed in $p d r \Delta$ cells treated with equisetin (at $10^{4}$ relative fluorescence unit, figure 4.4 ) displaying strong positive fluorescence intensity can be attributed to dying or dead cells readily taking up more MitoTracker stain and thus have on overall, higher fluorescent intensities. These results indicate that, in addition to the production of ROS, both TA-289 and equisetin are affecting mitochondria in a way that severely alters their morphology. 


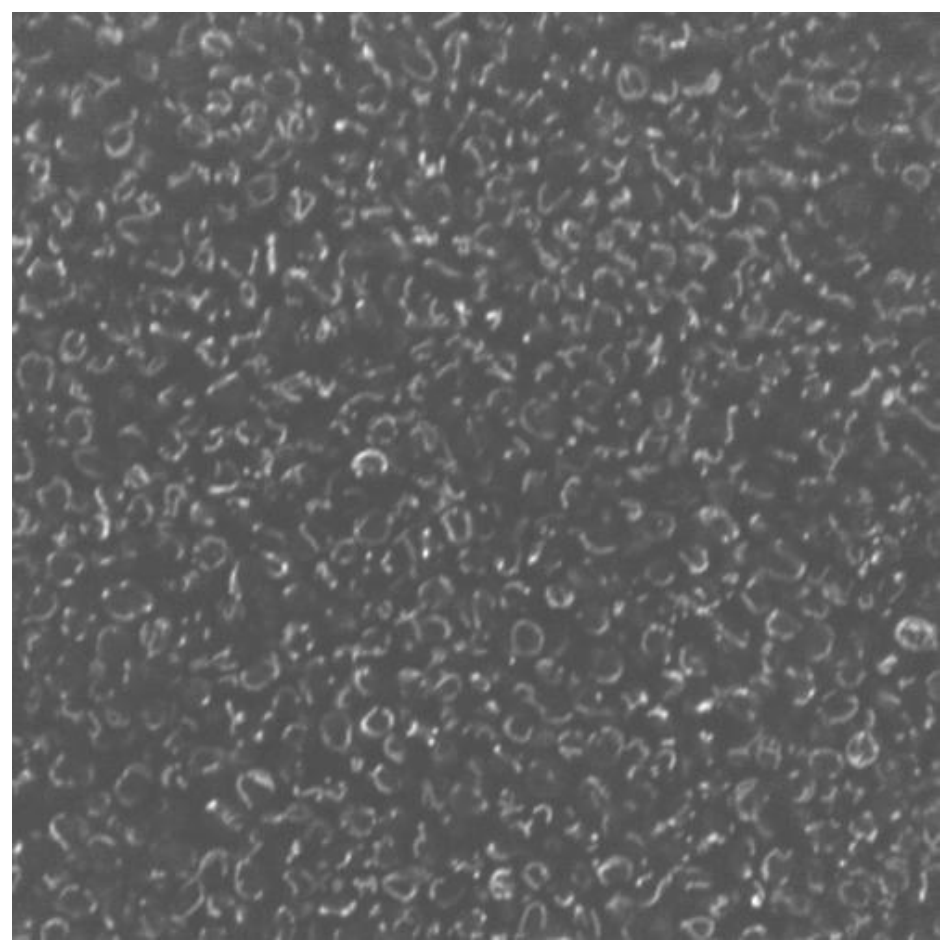

Figure 4.5.A. YOR356W (Cir2p-GFP) Untreated control cells. Normal mitochondrial morphology is displayed. Branched networks of mitochondria run along entire lengths of the cells.

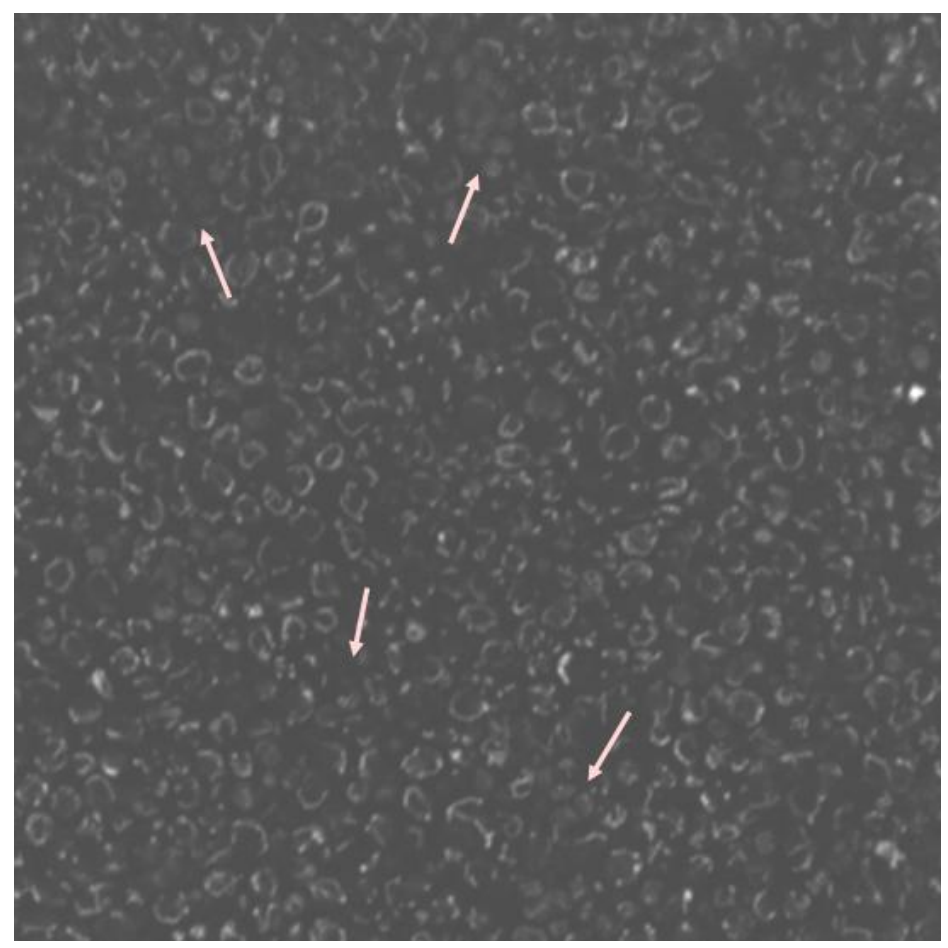

Figure 4.5.B. YOR356W (Cir2p-GFP) Cells treated with $50 \mu M$ TA-289. A slight difference in mitochondrial morphology is displayed. There are lesser amounts of normal branched networks of mitochondria, with more cells displaying diffused mitochondrial bodies across whole cells, and in some cells there are some slight punctate patterns (pink arrows) in a similar fashion to equisetin-treated cells. 


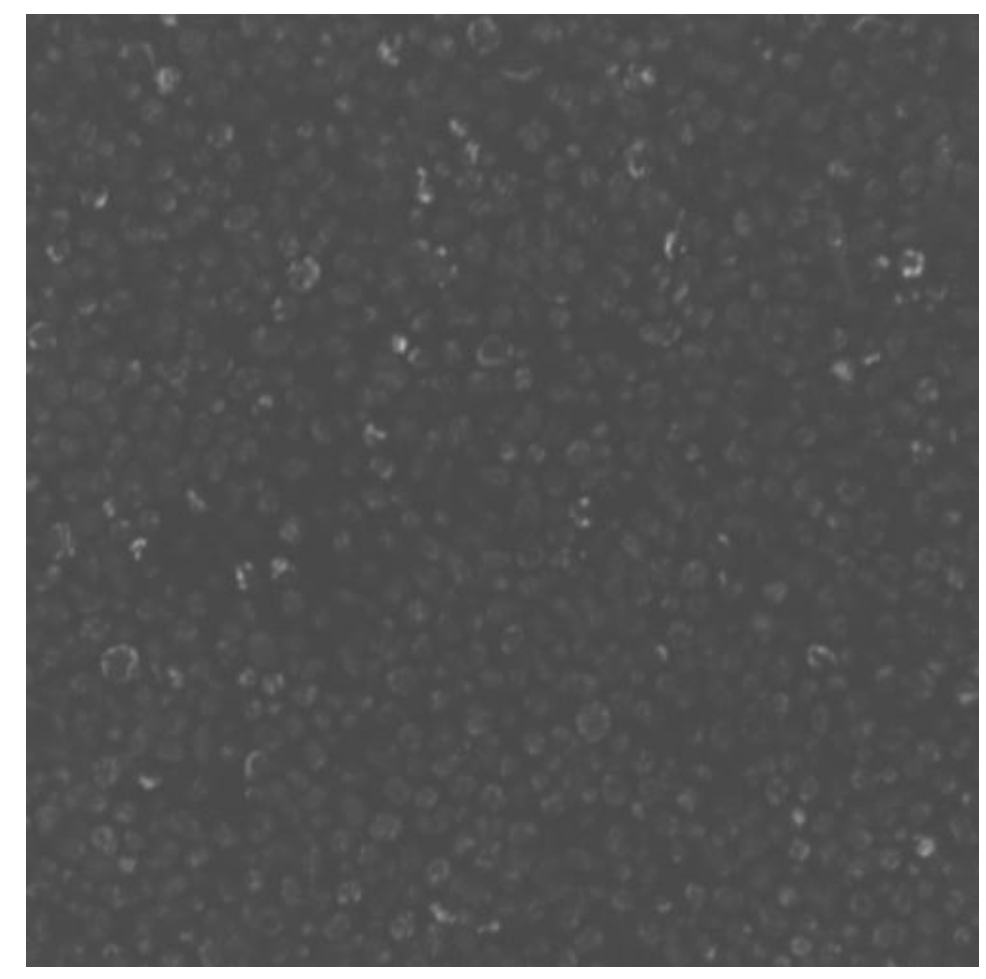

Figure 4.5.C. YOR356W (Cir2p-GFP) Cells treated with $50 \mu M$ equisetin. Branched mitochondrial networks are completely obliterated. Mitochondrial formation is almost punctatelike, diffused across whole cells. 


\subsubsection{High salt does not sequester potency of TA-289 and equisetin}

On the basis that a porin voltage-dependent channel might be involved in these mitochondrial effects, it was tested whether TA-289 and equisetin share a similar mechanism with SFK1, a putative Por1p (mitochondrial porin voltage-dependent anion channel) inhibitor (Butcher and Schreiber 2003). A dose response assay was performed with TA-289 and equisetin in the presence of high salt $(0.7 \mathrm{M} \mathrm{NaCl})$. As this concentration of $\mathrm{NaCl}$ slightly inhibits yeast cell growth, the assay was performed for $24 \mathrm{~h}$ instead of the standard $18 \mathrm{~h}$. Unlike SKF1, which inhibits in the absence of salt but not in its presence, the potencies of neither TA-289 nor equisetin were sensitive to the presence of $\mathrm{NaCl}$. In fact, the opposite was observed, where $\mathrm{NaCl}$ seemed to increase and/or prolong the potency of TA-289 and equisetin (Table 4.2). See Appendix 3.1 for dose-response figures.

Table 4.2. MIC values of TA-289 and equisetin in wt and pdrA strains in the presence or absence of $\mathrm{NaCl}$.

\begin{tabular}{|l|c|c|c|c|}
\hline \multirow{2}{*}{ Condition } & \multicolumn{2}{|c|}{ MIC $(\mu \mathrm{M})$ TA-289 } & \multicolumn{2}{c|}{ MIC $(\mu \mathrm{M})$ Equisetin } \\
\cline { 2 - 5 } & wt & pdr $\Delta$ & wt & pdr $\Delta$ \\
\hline No NaCl & $>100$ & 25 & 50 & 25 \\
\hline $0.7 \mathrm{M} \mathrm{NaCl}$ & 50 & 10 & 30 & 25 \\
\hline
\end{tabular}




\subsubsection{Testing candidate genes: Non-ETC genes}

A selection of strains with gene deletions that may be potential targets of TA-289 and equisetin were tested for sensitivity and/or resistance to either TA-289 or equisetin. The selected panel included genes involved in the mitochondrial ETC (a potential source of reactive oxygen species (ROS), and non-ETC related genes; genes hypothesised to be sources of ROS, genes involved in oxidative stress response, genes identified as putative targets of equisetin (König, Kapus et al. 1993), and genes associated with apoptosis. Non-ETC related genes showed no significant resistance ( $>1$, where 1 is indicated as wt growth) to TA-289 (Figure 4.6).

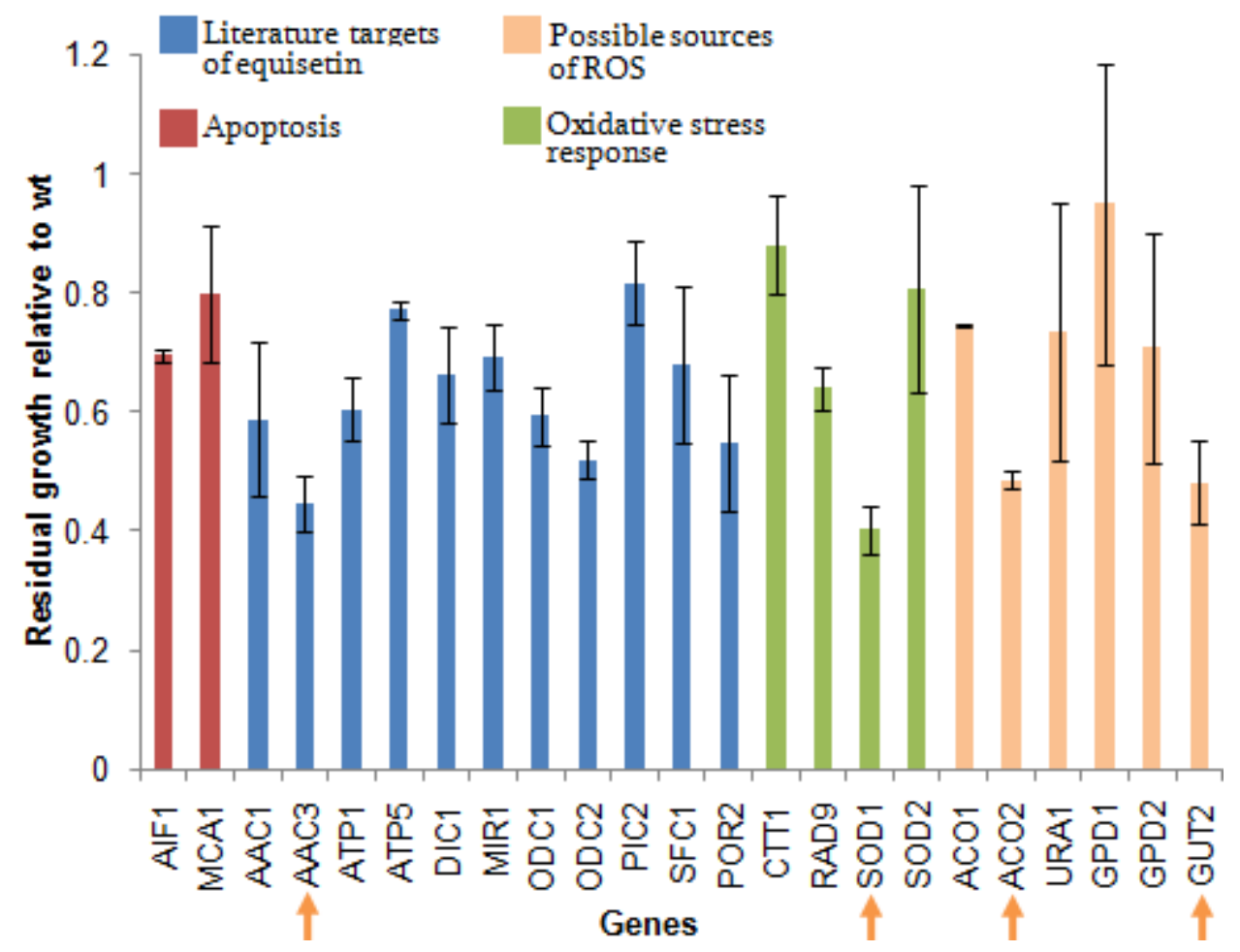

Figure 4.6. Sensitivity of non-ETC genes to TA-289. Deletion strains associated with ROS (pink), response to oxidative stress (green), apoptosis (red), and putative targets of equisetin (blue) were tested for sensitivity/resistance against $50 \mu \mathrm{M}$ TA-289, and residual growth relative to wt (y-axis) was plotted, where 1 on the y-axis indicates wt growth in $50 \mu \mathrm{M}$ TA-289. Orange arrows indicate genes that displayed more than half-fold sensitivity to TA-289 compared to wt. 
Genes encoding for proteins that may be a source of ROS production such as the aconitases $A C O 1$ and $A C O 2$, and dehydrogenases $U R A 1, G P D 1, G P D 2$, and GUT2, also displayed some sensitivity, where only aco2 $\Delta$ and gut $2 \Delta$ were a half-fold sensitive to TA-289. As such, it can be concluded that TA-289 does not cause the generation of ROS in cells through binding of putative non-ETC sources of ROS such as dehydrogenases, and that these genes do not encode the source for ROS.

Apoptotis-inducing factor AIF1 is translocated from the mitochondrion to the nucleus upon oxidative stress, leading to chromatin condensation and DNA degradation, i.e. pyknosis (Wissing, Ludovico et al. 2004). If aif1p was required for the cell death caused by TA-289 and equisetin, the aif1 $\Delta$ mutant strain will be resistant, however this was not observed, with the aif1 1 mutant showing the same sensitivity as BY4741 (wt). Yeast metacaspase $M C A 1$ is another positive regulator of apoptosis upon $\mathrm{H}_{2} \mathrm{O}_{2}$ treatment (Madeo, Herker et al. 2002). Given that the TA-289 resistant mutant generated in the previous study (chapter three, results 3.6.4) is also resistant to $\mathrm{H}_{2} \mathrm{O}_{2}$, it is possible that $M C A 1$ may be required for the cell death caused by TA-289, however wt sensitivity of the mca1 $\Delta$ mutant indicates otherwise.

Genes encoding for proteins involved in oxidative stress response could also be potential targets of TA-289 and equisetin, wherein binding of the enzymes may result in decreased tolerance to ROS (Gralla, Kosman et al. 1992). Additionally, the 
production of ROS observed upon TA-289 treatment may indicate that the compound may be targeting antioxidant functions that result in accumulation of ROS. These genes include catalase CTT1, superoxide dismutases $S O D 1$ and SOD2, and $R A D$ 9, involved in DNA damage repair. In this instance, the sod1 $\Delta$ mutant was sensitive to TA-289, which suggests that 1$)$ superoxide anions $\left(\cdot \mathrm{O}_{2}-\right)$ are being generated because 2) the sod $1 \Delta$ mutant is unable to remove these free radicals and is thus more sensitive compared to wt. However, the sod2 $\Delta$ mutant is not sensitive. Whether this is an artefact of the assay conducted, or because SOD2 is not as highly expressed as $S O D 1$ under normal physiological conditions and so the sod2 $\triangle$ mutant is not as compromised as the sod1 $1 \Delta$ mutant, it is unclear.

Yeast homologues of the putative gene targets of equisetin were also tested, including ATP-associated genes $A A C 1$ and $A A C 3$ (ADP/ATP translocators; $A A C 1$ is expressed under aerobic conditions and $A A C 3$ is expressed under anaerobic conditions), and ATP1 and ATP5 (subunits of F0-F1 ATP synthase), oxo/dicarboxylate carriers $O D C 1, O D C 2$, and $D I C 1$, phosphate carriers $M I R 1$ and PIC2, and succinate/fumarate carrier SFC1. The mitochondrial porin POR2 was included in this screen as Por1p was indicated as the target of mitochondrial inhibitor SFK1 (Butcher and Schreiber 2003), and ATP5 has been shown to be the target of oligomycin (Bertina, Steenstra et al. 1974). These groups of genes showed no dramatic increased in sensitivity to TA-289 aside from $A A C 3$ - the differing 
sensitivites of aac3 $\Delta$ and aac1 $\Delta$ mutants to TA-289 can be explained by their expression levels under anaerobic conditions i.e. glucose, where the expression of $A A C 3$ in glucose plays a role in maintaining cell viability and respiration, and thus the aac3 $\Delta$ mutant is sensitive to TA-289. However $A A C 1$ is not expressed in glucose (the conditions used in this assay), and as such the aac1 $1 \Delta$ mutant will most probably not be as sensitive to TA-289 as that of the aac3 $\Delta$ mutant, as it is observed in figure 4.6. The lack of a pattern of sensitivity to TA-289 in these group of genes suggest that 1) neither TA-289 nor equisetin act in a similar way to SFK1, or oligomycin (as neither the por $2 \Delta$ or atp $5 \Delta$ strains displayed resistance) and 2) that possible targets of equisetin (and by association, TA-289) highlighted by König et. al. may not be the true targets of TA-289 and equisetin in yeast.

\subsubsection{Testing candidate genes: ETC genes}

The mitochondrion is a major source of ROS. The generation of ROS and altered mitochondrial morphology upon treatment of TA-289 and equisetin suggests a possible site of action at the ETC. As such, select genes from each ETC complex were tested with three different concentrations of equisetin and their sensitivity/resistance to the compound was measured. Figure 4.7 shows gene deletions associated with complex I of the ETC (NED1 and NDI1), and complex II (SDH1, SDH2, and EMI5). NDE1 and $S D H 2$ showed significant sensitivity to lower concentrations of equisetin ( $1 \%$ significance). However, SDH2 displayed strong 
resistance (two-fold resistance at the highest concentration). NDI1 and EMI5 were no different to wt growth in equisetin.

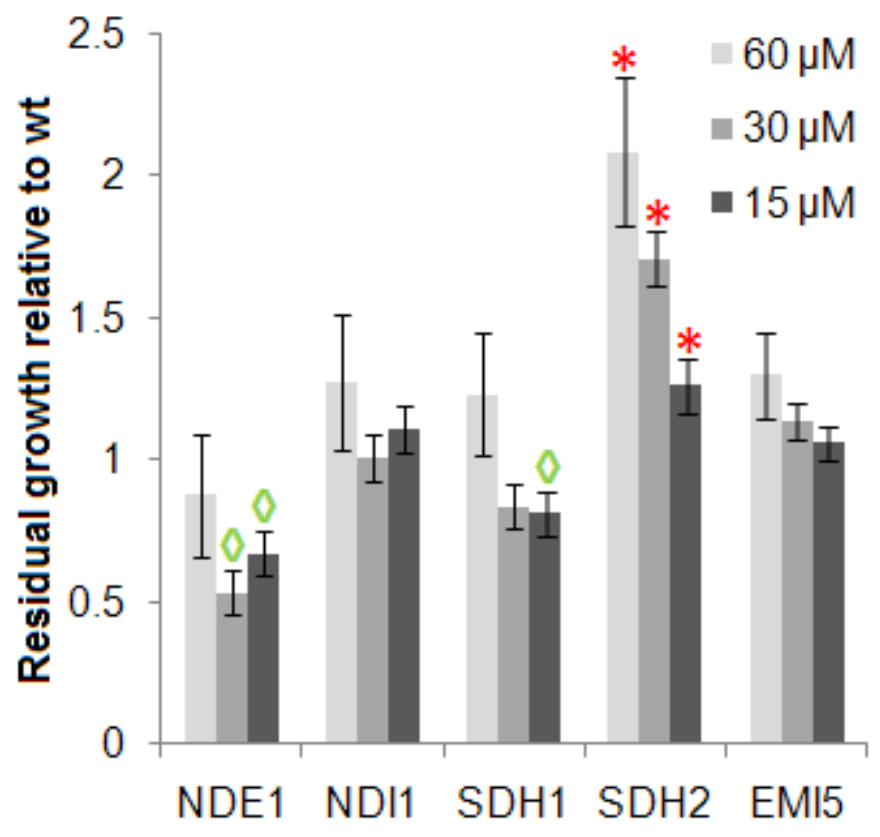

Figure 4.7. Sensitivity of complex I and complex II genes to equisetin. Deletion strains of genes involved in complex I (nde1 $\Delta$, ndi1 $\Delta$ ) and complex II ( $s d h 1 \Delta, s d h 2 \Delta$, and emi5 $\Delta$ ) were tested for sensitivity/resistance against $60 \mu \mathrm{M}, 30 \mu \mathrm{M}$, and $15 \mu \mathrm{M}$ equisetin, and residual growth relative to wt (y-axis) was plotted. Resistance (red asterisk) and sensitivity (green diamond) of $1 \%$ significance are denoted. 
Figure 4.8 below shows gene deletions associated with complex III, including genes required for ubiquinone (coenzyme $\mathrm{Q})$ biosynthesis (COQ1 and COQ2).

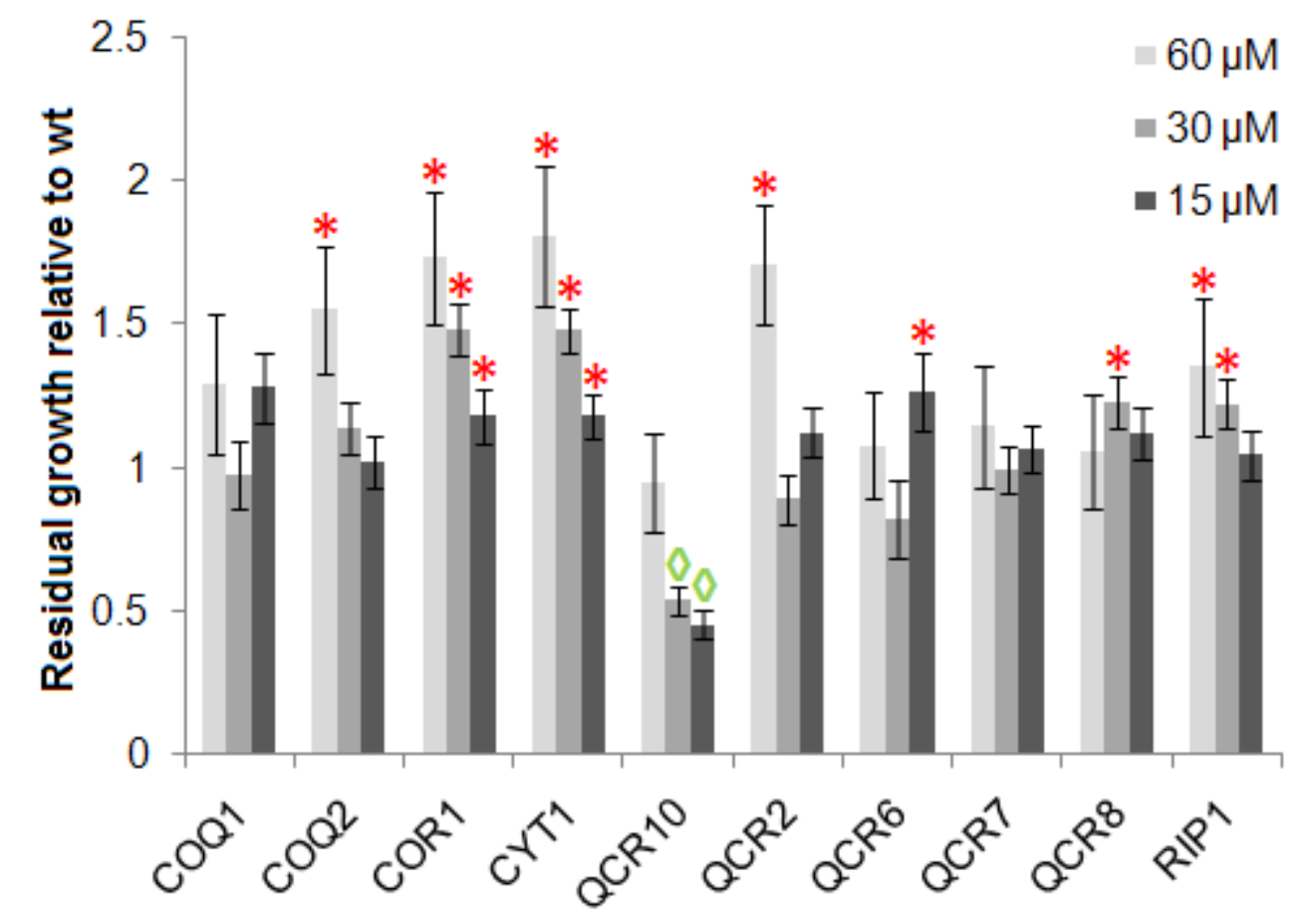

Figure 4.8. Sensitivity of complex III genes to equisetin. Deletion strains of genes involved in complex III were tested for sensitivity/resistance against $60 \mu \mathrm{M}, 30 \mu \mathrm{M}$, and $15 \mu \mathrm{M}$ equisetin, and residual growth relative to wt (y-axis) was plotted. Resistance (red asterisk) and sensitivity (green diamond) of $1 \%$ significance are denoted.

COR1 (core subunit of complex III), and CYT1 (cytochrome c1), displayed significant resistance at all concentrations of equisetin tested. RIP1 (ubiquinol to cytochrome c electron transport) also displayed resistance at 60 and $30 \mu \mathrm{M}$ equisetin. The QCR genes (subunits II of complex III) also displayed resistance at the concentrations of equisetin tested, however QCR10 showed significant sensitivity at 30 and $15 \mu \mathrm{M}$, but normal growth at $60 \mu \mathrm{M}$ equisetin. Notably, only QCR10 
displayed significant sensitivity, with all other genes tested displaying either resistance or normal growth $(\approx 1$, wt growth).

Figure 4.9 below shows gene deletions associated with complex IV, cytochrome c oxidase, and cytochrome $c$ translation (PET122 and PET494).

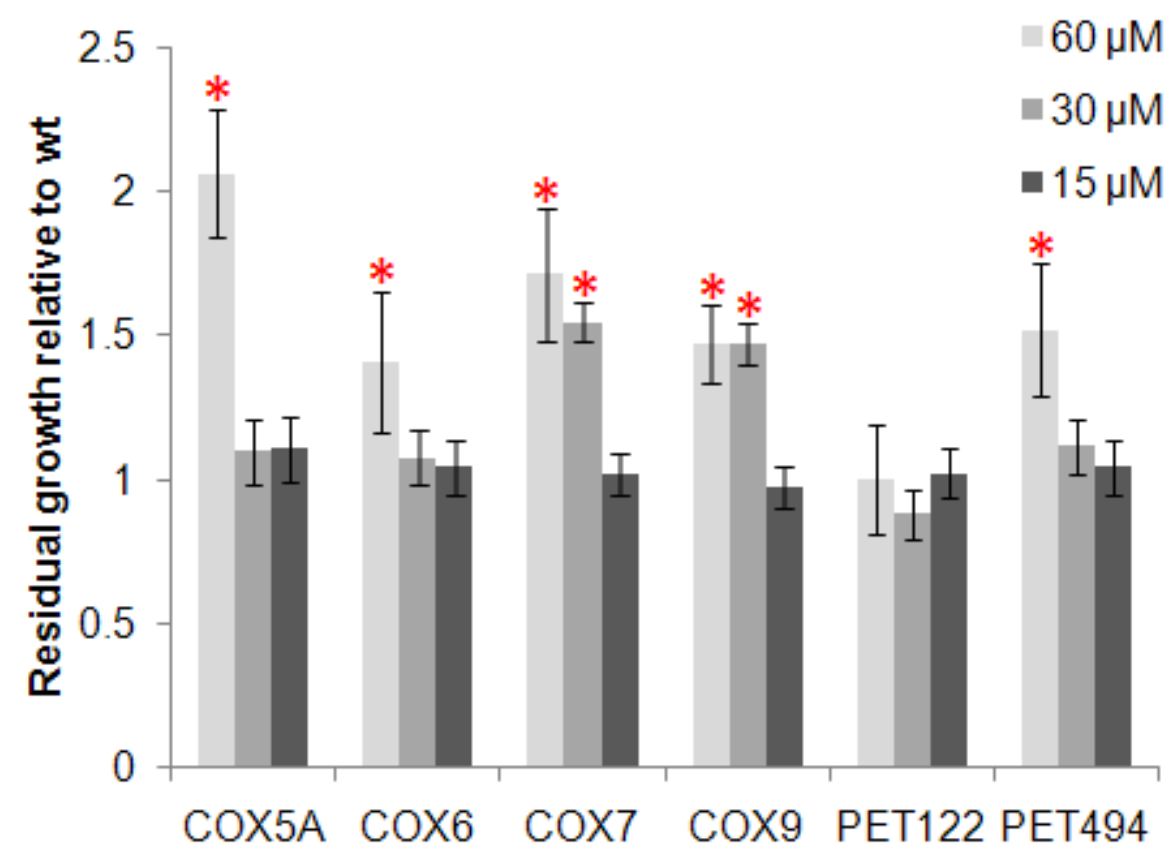

Figure 4.9. Sensitivity of complex IV genes to equisetin. Deletion strains of genes involved in complex IV were tested for sensitivity/resistance against $60 \mu \mathrm{M}, 30 \mu \mathrm{M}$, and $15 \mu \mathrm{M}$ equisetin, and residual growth relative to wt (y-axis) was plotted. Resistance (red asterisk) of $1 \%$ significance are denoted.

COX7 and $\operatorname{COX} 9$ (subunits VII and VIIa of complex IV) displayed resistance at 30 and $60 \mu \mathrm{M}$ equisetin. COX6 (subunit VI), whose expression is regulated by oxygen levels (Wright, Simpson et al. 1995), also displayed resistance to equisetin, at the highest concentration tested. $C O X 5 A$, predominantly expressed during aerobic 
growth, is most resistant at the highest concentration of equisetin tested, displaying two-fold resistance. Mitochondrial translation activators PET122 and PET494 displayed normal growth in all concentrations, with PET494 showing resistance to equisetin only at $60 \mu \mathrm{M}$. Here, all deletion strains of genes encoding for proteins involved in complex IV of the ETC displayed either normal growth $(\approx 1$, wt growth) or were strongly resistant. 


\section{$\underline{4.5 \text { Discussion }}$}

\subsubsection{Effects of TA-289 and equisetin on the redox state of yeast}

$S$. cerevisiae are free-living unicellular organisms, and as such the eukaryote must endure whatever stressors to which it is exposed. Endogenous stress can occur in many ways, one of them being the production of reactive oxygen species (ROS), natural by-products of respiration in all aerobic organisms. Oxidative stress as a result of ROS production will cause damage to major cellular components such as proteins, lipids, and DNA, and ultimately, where a cell is unable to maintain its redox state, cell death (Salmon, Evert et al. 2004). The work presented here shows that the novel fungal metabolite TA-289 and structurally related equisetin both significantly affect the redox state of yeast, likely so by the induction of oxidative stress.

Oxidant sensitive probes such as 2,7-dichlorofluorescein diacetate (DCF) are often used to measure ROS levels within cells. In this instance, the cell permeable dye propidium iodide was also used as a counter stain to DCF. TA-289 caused a marked increase in ROS production in cells within 30 mins treatment, and by the hour, the majority of the cell population were either dead (stained positive for propidium iodide) or dying from elevated ROS production (stained positive for both DCF and propidium iodide). Equisetin treated cells displayed similar characteristics, however over $90 \%$ of the cell population were dead within 30 mins of treatment, with the 
rest staining positive for ROS production. This could be attributed to the fact that equisetin is much more potent than TA-289, and so is more fast-acting on yeast cells. This evidence is supported by the cell death assays conducted in glucose as a carbon source, where TA-289 and equisetin kill within the first hour of treatment (wt and $p d r \Delta$ cells for equisetin, $p d r \Delta$ cells only for TA-289), while a known oxidant, $\mathrm{H}_{2} \mathrm{O}_{2}$, takes up to three hours before yeast are severely compromised. This in turn may explain why cells treated with $\mathrm{H}_{2} \mathrm{O}_{2}$ in the ROS-detection assays had only slight elevated ROS production, where the level of ROS produced will most likely increase given enough exposure time to $\mathrm{H}_{2} \mathrm{O}_{2}$. It is likely that if cells were visualized after 15 mins exposure to equisetin instead of 30 or 60 mins, more ROS-producing cells may be detected with a lesser population of dead cells. Ultimately the best course of determining ROS production would be a time-course analysis with tighter intervals between each visualization to establish elevated production of ROS, and most importantly, that this event appears to precede yeast cell death, or at least precede the increase in cell wall permeability.

Interestingly, a small percentage of cells in the untreated control were stained positive for ROS production. Additionally, there was also a population of dead cells as shown by positive-staining with propidium iodide. It is not uncommon however that in any given population of cells, there will always be a proportion of cells that are dead, or are dying and thus may produce more ROS naturally. The cell 
membrane is a target of ROS, and a damaged membrane will most likely become more permeable. Propidium iodide is able to cross permeablized cell membranes (often an indication of dead cells) (Davey and Hexley 2010), therefore it is possible that cells treated with TA-289 and equisetin stain positive for propidium iodide due to compromised cell membranes, not because the cells are dead from the effects of the compounds. Moreover, cells that stain positive for propidium iodide have been shown to be able to repair the cell membrane if given enough incubation time to do so, and so subsequent exposure to propidium iodide post-repair do not stain positive (Davey and Hexley 2010). Nevertheless, the proportion of cells that are able to repair their membranes enough to exclude propidium iodide is relatively minor, and the inability of $p d r \Delta$ cells to recover from treatment with TA-289 and equisetin as observed in the CFU assays (chapter two, result 2.5.1) and the time course analyses (chapter two, result 2.5.4) is strong indication that pdrA cells are severely irreversibly growth inhibited in their inability to expel the compounds, not in their inability to repair the cell membrane (as wt cells were able to recover from treatment with TA-289 and slow recovery from equisetin). 


\subsubsection{The effects of TA-289 and equisetin on yeast mitochondria}

The mitochondrion is often known as the powerhouse within a cell, where ATP generated within this organelle is transported to various cellular components in the cell that rely on ATP to function. The mitochondrial transmembrane potential $\left(\Delta \Psi_{\mathrm{m}}\right)$ is essential not only for ATP production, but also the regulation of metabolite transport, and is required for mitochondrial protein import (Ricci, Waterhouse et al. 2003). Mitochondria are also a major source of ROS production, where premature electron leakage from the ETC during respiration results in the formation of free radicals such as the superoxide anion $\left(\cdot \mathrm{O}_{2}{ }^{-}\right)$, and the hydroxyl radical $(\cdot \mathrm{OH})$. The generation of ROS can lead to a collapse in the $\Delta \Psi_{\mathrm{m}}$, where cellular damage caused by ROS and prolonged exposure will ultimately lead to cell death. To this end, the site of action of both TA-289 and equisetin has been indicated at the mitochondria. From results attained in these studies, it is strongly suggested that TA-289 and equisetin share the same mechanisms-of-action, wherein both compounds cause a deleterious production of ROS, leading to yeast cell death that is speculated to be mediated and or initiated by/in the mitochondrion.

Following ROS-detection assays, studies into the effect of TA-289 and equisetin on the mitochondria by staining with whole mitochondria stain, MitoTracker ${ }^{\odot}$ Green FM revealed that cells treated with either TA-289 or equisetin exhibited significantly reduced uptake of MitoTracker ${ }^{\odot}$ Green FM. The MitoTracker probe is a mitochondrion-selective dye containing a thiol-reactive chloromethyl moiety. Once 
inside the mitochondria, MitoTracker reacts with accessible thiol groups on peptides and proteins to form a fluorescent conjugate with proteins in the mitochondria (Molecular Probes, Invitrogen Corporation, CA, USA). Although a reduced staining of MitoTracker is observed in treated cells, this is not necessarily due to a disruption in the $\Delta \Psi_{\mathrm{m}}$, as MitoTracker will stain mitochondria regardless of $\Delta \Psi_{\mathrm{m}}$.

Loss of MitoTracker staining in the mitochondria could be a result of oxidation of accessible thiol groups by ROS produced as a result of treatment with TA-289 or equisetin, and where there are less thiol groups for MitoTracker to react with and fluoresce; fluorescent intensity is lessened compared to untreated controls. ROS has been shown to alter mitochondrial membrane, a consequence of lipid peroxidation and thiol oxidation (Batandier, Fontaine et al. 2002). To determine whether this is indeed happening within the mitochondria, staining with MitoTracker prior to treatment with either TA-289 or equisetin should not result in the sequestering of fluorescence intensity as MitoTracker would have already bound to accessible thiol groups. Additional staining with mitochondria-selective probes that are sensitive to changes in the $\Delta \Psi_{\mathrm{m}}$ will establish whether effects of TA-289 and equisetin result in a collapse of the $\Delta \Psi_{\mathrm{m}}$.

Assessment of mitochondrial morphology using a Cir2p-GFP fusion protein expressing strain localized in the mitochondria showed that TA-289, and especially 
equisetin, caused gross morphological changes in the yeast mitochondria. Under normal physiological conditions, yeast mitochondria form a network of tubular membranes located at the cell cortex, on the inner face of the plasma membrane (Hoffmann and Avers 1973) arising from a single, branched mitochondrion that is continuous between the mother cell and daughter cell and throughout a large portion of the cell cycle. The mitochondrion is actively transported by cytoskeletal components to the emerging bud during $\mathrm{G}_{1}-\mathrm{S}$ phase (Stevens 1981).

The tubular networks observed in untreated cells were partially disrupted in TA289-treated cells, and completely disrupted in equisetin-treated cells within $1 \mathrm{~h}$ in what appears to be fragmentation or punctate patterns. Again, the significant effects induced by equisetin compared to that of TA-289 are most likely due to its greater potency in yeast; equisetin is more hydrophobic than TA-289 and thus will be more permeable. Currently, it is unclear whether elevated ROS production causing cellular damage is a result of the changes in mitochondrial morphology, or whether it is the production of ROS that causes morphological changes in the mitochondria. Nevertheless, these results clearly show TA-289 and equisetin are affecting yeast mitochondria, potentially collapsing the $\Delta \Psi_{\mathrm{m}}$, where loss of ATP synthesis and ROS generation are both contributing factors in irreversible growth inhibition. 


\subsubsection{Potential molecular targets of TA-289 and equisetin: The ETC}

To date, a number of suggestions have been made as to the target of equisetin. Equisetin was shown to potently inhibit the activity of purified HIV-1 integrase (Singh, Zink et al. 1998), and SecA activity in vitro (Sugie, al et al. 2002), and the substrate anion carriers of the rat mitochondrial ETC (König, Kapus et al. 1993) have also been suggested. Patham et. al. also showed that equisetin inhibited the translocation of proteins to the ER. In $S$. cerevisiae, there is no homologue of HIV-1 integrase, however observed biological activity in yeast suggests either a different mechanism-of-action of equisetin (and in relation, TA-289), or the likelihood of multiple targets by the compound. Yeast homologues of mammalian translocons include $S B H 1$ and $S B H 2$; $s b h 1 \triangle$ cells have been shown to be sensitive to oxidative stress-inducing compounds, CTBT (7-chlorotetrazolo[5,1-c]benzo[1,2,4]triazine) (Batova, Klobucnikova et al. 2010), and glycoaldehyde (Jayakody, Hayashi et al. 2010).

SBH1 and SBH2 are subunits of the SEC61 translocon complex, and as in bacteria, are involved in translocation of proteins across the ER lumen and into the mitochondria (Boisrame, Chasles et al. 2002). At present, it is unclear whether inhibition of SecA activity will cause ROS production, however, the process by which SecA carries out protein translocation is ATP-dependent (Sugie, al et al. 2002), and it is possible that activity is diminished through interference of ATP by equisetin (notably, ATP uptake is inhibited in another study conducted by König et. 
al.). The inhibition of substrate anion carriers $\left(\mathrm{P}_{\mathrm{i}}\right.$, succinate, and ATP) of the isolated rat mitochondria, in combination with reports that show the ability of equisetin to activate inorganic pyrophosphatase (ppa2p) (Lundin, Deopujari et al. 1992), suggests that equisetin acts on the mitochondria in yeast also.

All the deletion strains comprising genes involved in the ETC that showed significance resistance to equisetin, were identified as sensitive to compounds known to induce oxidative stress, including 2-phenyl-3-nitroso-imidazo[1,2a]pyridine (Yu, Lopez et al. 2008), DTT (1,4-dithiothreitol) (Rand and Grant 2006), 3-amino-1,2,4-triazole (Alamgir, Erukova et al. 2010), and 2,4diacetylphloroglucinol (Kwak, Han et al. 2011). This suggests that the mechanism by which equisetin acts on the cell is different to the described compounds. In saying that however, several genes associated with the ETC that displayed resistance to equisetin as observed in figures $4.7-4.9$, were sensitized to TA-289 in the chemical genomic profiling screens performed in chapter two. This may be a result of cellular tolerance, where at higher concentrations (i.e. $30 \mu \mathrm{M}$ and $60 \mu \mathrm{M}$ equisetin as conducted in this study) cells significantly increase their buffering capacity to counteract the effects of the drug.

Putative genes identified as potential targets for equisetin as described by König et. al. were also tested in this study. König et. al. showed that equisetin inhibited uptake 
of $\mathrm{P}_{\mathrm{i}}$, succinate, and ATP, therefore suggesting genes involved in the import/export of these molecules as targets. Yeast deletion strains of genes showing homology to the putative targets described by König et. al. were tested. These were phosphate carriers $M I R 1$ and $P I C 2$, succinate/fumarate carrier SFC1, and oxo/dicarboxylate carriers $O D C 1, O D C 2$, and $D I C 1$. No one deletion strain of any of these genes described were significantly sensitive to equisetin at all concentrations tested, suggesting that these are not the targets of equisetin.

AIF1 is a mitochondrial pro-apoptotic gene responsive to oxidative stress, the deletion of which increases resistance to oxidative stress (Wissing, Ludovico et al. 2004); its deletion strain aif $\triangle$ showed no significant sensitivity. SOD1 and SOD2, superoxide dismutases regulated in response to oxidative stress, the deletion of which often result in increased sensitivity to ROS-inducing agents (Tan, Teo et al. 2009)). As with $A I F 1$, sod1 1 and sod2A showed little significant sensitivity or resistance, with only the sod $1 \Delta$ mutant displaying a two-fold sensitivity compared to wt. These results indicate that equisetin may act somewhere in the mitochondrial ETC, or its target(s) associates with the ETC, as deletion strains of genes not associated with ETC displayed varying resistance and sensitivity, most likely as a result of indirect effects of equisetin binding to its target. 
A selection of genes associated with complexes I through IV of the ETC were tested with equisetin. Among these, complex III- and complex IV-associated genes were the only group of genes to show either differential sensitivity compared to wt, or were resistant to equisetin (with the exception of $Q C R 10$ at complex III), indicating a possible site of action at either of these complexes in the ETC. It was also noted that deletion strains that were resistant to equisetin displayed strongest resistance at the highest concentration tested $(60 \mu \mathrm{M})$ compared to 15 and $30 \mu \mathrm{M}$. This may be a result of cellular response to oxidant dose, where genes involved in detoxification have been shown to be down-regulated at low oxidant doses, but significantly upregulated at higher doses (Alic, Felder et al. 2004), leading to greater resistance. This possibility should be amenable to testing by expression microarrays to test for specific gene transcription. Likewise, what may seem as strong resistance could be a result of the wild-type strain being severely growth inhibited at higher concentrations, and thus compared to wild-type growth, resistance appears more significant.

Currently, it is unclear whether complex IV is capable of producing ROS, however inhibition of complex IV by equisetin and TA-289 may cause premature leakage of reducing electrons from cytochrome $c$. Additionally, it is possible that the target of equisetin may be downstream of the ETC complexes tested, owing to the pattern of resistance across all four complexes. Similar to paraquat (Tan, Teo et al. 2009), 
equisetin and TA-289 may act as alternative terminal receptors of the ETC, becoming a free radical in itself and generating more ROS. A more probable scenario, taking into consideration the observations of resistance displayed by genes associated with complex III and complex IV (figures 4.6-4.9), is that 1) resistance is observed at complex III because gene deletions involved in that complex result in reduced ROS production rate, and 2) resistance is observed at complex IV because gene deletions involved in complex IV result in an electron flow "back-up" to complex III which, notably, has not been shown to cause a burst of ROS production (Andreyev 2005). There is no evidence for ROS production at complex IV, and in this case, the source of ROS production upon treatment with either TA-289 or equisetin may be generated from complex III of the ETC, a well-known producer of ROS. Notably, not all genes involved in the ETC were tested, and as such the sensitivity of these deletion strains may be worth testing against TA-289 and equisetin in the future.

To this end, TA-289 and equisetin have been shown to elicit ROS production in yeast, wherein elevated ROS levels leads to either irreversible growth inhibition or cell death (as observed by the ROS-detection and propidium iodide staining assays). Treatment with TA-289 and equisetin also cause severe morphological changes in mitochondrial morphology, most probably as a result of ROS production. Currently, it can only be speculated that due to gross morphological changes in the 
mitochondria, there is loss of $\Delta \Psi_{\mathrm{m}}$, however equisetin has been previously demonstrated to not have significant effects on the $\Delta \Psi_{\mathrm{m}}$ (König, Kapus et al. 1993). The disparities between resistance and sensitivity of non-ETC genes tested in this study suggest that TA-289 and equisetin may act at the ETC, where the pattern of resistance observed between genes involved in complexes III and IV indicate a potential site-of-action at those complexes. However, evidence showing equisetin inhibits the translocation of post-translation proteins to the ER (Patham, Duffy et al. 2009) further indicates a possible action outside of the ETC, or a target that is tightly involved in both the mitochondria and the ER. 


\section{FINAL DISCUSSION}

\subsection{Mitochondrial-mediated ROS production in yeast}

The novel fungal metabolite TA-289, and a structurally related compound equisetin, display potent growth inhibitory effects against $S$. cerevisiae at micromolar concentrations. Further characterization of the mechanism by which these two compounds elicit their effects on yeast have been attempted in this study using various genome-wide tools available for $S$. cerevisiae. Evidently, the late-phase cell cycle block and chemical genomic profiling of TA-289 presented in chapter two, as well as previous reports indicating the effects of equisetin on the mitochondria, lead us to hypothesise that both compounds were eliciting their effects by causing elevated ROS production leading to oxidative stress, triggering either programmed yeast cell death or irreversible growth inhibition.

Production of ROS was detected in cells after treatment with TA-289. Treatment with TA-289 also resulted in cells with significantly damaged and permeable cell membranes, a characteristic of dead cells. In this instance however, it is believed that cells treated with TA-289 and equisetin are susceptible to propidium idodidestaining as a result of ROS generation which causes damage to cellular components, including the cell membrane. Thus, a more definite marker for cell death will need to be applied to determine whether cells die from treatment with TA-289 and equisetin, or are simply irreversibly growth inhibited as was noted with isolated rat 
mitochondria (König, Kapus et al. 1993). Moreover, the reagent used to detect the presence of ROS in cells does not discriminate between different types of ROS. However, taking into consideration the late phase cell cycle block observed in the cell cycle analysis in chapter two, it is possible that the highly reactive hydroxyl radical, $\cdot \mathrm{OH}$, is generated upon TA-289 and equisetin-treatment, as the production of these radicals have been known to cause a cell cycle block at $\mathrm{G}_{2}$ phase (Perrone, Tan et al. 2008). Whether the hydroxyl radical is generated as a direct result upon treatment with TA-289 and equisetin, or whether it is through the generation of other free radicals such as superoxides and peroxides (these three ROS are often linked by either spontaneous or enzymatic dismutation (Batandier, Fontaine et al. 2002)), remains unclear.

The growth inhibition of yeast cells by TA-289 and equisetin during fermentative growth indicate that the target(s) of TA-289 and equisetin must be present even under these conditions. If the target(s) is located or is associated with mitochondrial pathways, and if the source of ROS is indeed from the mitochondria, then it is presumed that there is the presence of some basal level of respiration, even when yeast are fermenting, since ROS is produced upon treatment with TA-289 and equisetin. Thus, upon target-binding, premature electron leakage from the ETC causes ROS, gross morphological changes in the mitochondria, and presumably a decrease in cellular ATP. Apoptosis is a process that requires ATP (Ricci, 
Waterhouse et al. 2003). Although yeast display similar hallmarks of mammalian apoptosis such as pyknosis (as it is observed in cells treated with TA-289 and equisetin), it is unclear whether the requirement for yeast apoptosis is dependent upon ATP as it is in mammalian apoptosis (Madeo, Herker et al. 2004; Madeo, Carmona-Gutierrez et al. 2009). If ATP-synthesis is indeed compromised and/or depleted upon TA-289 and equisetin treatment, but yeast cells are still displaying apoptotic markers like pyknosis and also staining positive for cell death (propidium iodide), the requirement for yeast apoptosis in this instance may indeed be different to mammalian apoptosis.

The difference in growth inhibition and/or adaptive resistance observed between treatment with TA-289 and equisetin in fermentable and non-fermentable carbon sources suggest either 1) when yeast are fermenting, the target(s) of TA-289 and equisetin are lowly expressed, requiring less drug to bind to the target(s) and thus the cell is overwhelmed; or 2) under active respiration (non-fermenting conditions), the target(s) is presumably more expressed as it is required for respiration, and the increase in expression of drug target will require more drug to bind to the target and cause an effect. Moreover, when a cell is actively respiring, the cell will most likely produce more ROS compared to fermentative conditions, and thus antioxidant capacities are up-regulated (Drakulic, Temple et al. 2005). The combined effect of the presence of more drug target, and the increased antioxidant capacity may 
explain the increased resistance to TA-289 and equisetin observed in the cell death assays performed in chapter two, where TA-289 and equisetin do not appear to kill $p d r \Delta$ yeast in glycerol-ethanol, but the compounds killed wt yeast (figures 2.14 and 2.15). The seemingly increased sensitivity of wt to TA-289 and equisetin is evidence that respiration plays an important role in cell survival upon treatment with these two compounds. The resistance observed in the cell death assays by the $p d r \Delta$ cells can be explained by these mutants having higher basal level of ROS under normal respiratory conditions, and thus an overall greater antioxidant capacity to be able to buffer the inhibitory effects of TA-289 and equisetin. Longer exposure time performed for cell death assays in glycerol may determine whether the inhibitory effects of TA-289 and equisetin will eventually overcome the initial adaptive resistance mechanisms observed in the $p d r \Delta$ cells.

Often, it can be difficult to ascertain whether the production of ROS precedes or is a result of cell death and/or growth inhibition. Notably, the resistant mutant generated in this study showed that resistance to TA-289 could be achieved by the mutant strain possessing greater antioxidant capacity due to its higher basal level of ROS production. This was determined where the mutant also displayed resistance to a known oxidant, $\mathrm{H}_{2} \mathrm{O}_{2}$. These findings also provide evidence that induction of ROS by TA-289 is a separate process and precedes cell death, as the mutant with upregulated antioxidant capacity has the ability to grow at concentrations of TA-289 at 
which wild-type succumbs. This interpretation is supported by the time-course analyses of cells treated with TA-289 display elevated ROS production prior to cells becoming permeable to propidium iodide. Most importantly, while it has been established that ROS production caused by TA-289 and equisetin may lead to cell death and/or growth inhibition, it is more challenging to determine the source of ROS. In light of the assays performed with the selected genes involved in the ETC complexes, the pattern of resistance observed across the deletion strains of complex III suggest a possible source of ROS at that complex when treated with TA-289. It is important to note however, that the site of ROS production does not necessarily equate to the binding-site (i.e. the target) of TA-289 and equisetin.

Many of the results concerning genetic interactions reported here can be explained by processes linked to the mitochondria or genes involved in mitochondrial inheritance. Gene groupings identified in the chemical genomic profiling (both homozygous profiling and haplo-insufficiency profiling) included mitochondrialrelated processes, DNA damage and oxidative stress, all of which are affected directly by the generation of ROS induced by TA-289, and most probably equisetin, as both compounds have displayed very similar mechanisms so far. In the case where the mitochondrion is the site of action for TA-289 and equisetin, it will be the first organelle to be affected by ROS as these will most likely be generated within the organelle itself. A large number of genes associated with RNA 
polymerase II regulation (both negative and positive regulation), and transcription from RNA polymerase II promoter were also identified. Studies have shown that the regulation of RNA polymerase II plays a role in stress response and cell fate (Pelechano, Jimeno-Gonzalez et al. 2009; Jouvet, Poschmann et al. 2010). As RNA polymerase II is an integral complex in gene expression, for a cell that is unable to repair damaged proteins or DNA, or transcribe mRNA encoded for the response of cellular stress and damage, it will most likely be sensitive to TA-289 and equisetin. This observation does not argue so much for any particular specificity for both compounds as it does that cellular response to TA-289 and equisetin is a living cell response i.e. general up-regulation of gene expression.

Additional processes affected also included those that occurred in the endoplasmic reticulum (ER) and endosome trafficking and vesicular transport, where the close contact sites between mitochondria and ER will most likely be exposed to ROS leakage from the mitochondria. As ROS can interact with many different proteins and lipids (especially so in the case of the hydroxyl radical $\cdot \mathrm{OH}$ ), damage to these cellular components will cause an ER stress response, and in the event where stress is prolonged and the cell is unable to traffic or repair damaged proteins, the result is detrimental. Moreover, in the event where mitochondria-mediated ion signalling such as $\mathrm{Ca}^{2+}$ which plays a role in cellular fate, is affected by TA-289 and equisetin, the resulting dysfunction in ion dynamics between the mitochondria and ER can be 
deleterious to the cell (Decuypere 2011). Equisetin was previously shown to inhibit $\mathrm{Ca}^{2+}$ uptake in isolated rat mitochondria (König, Kapus et al. 1993). This, combined with the identification of genes associated with $\mathrm{Ca}^{2+}$ signalling in the chemical genomic profiling of TA-289, is further evidence that TA-289 and equisetin may share the same mechanism-of-action. Additionally, it illustrates that the effects of TA-289 and equisetin on yeast mitochondria could also result in aberrant $\mathrm{Ca}^{2+}$ signalling. A dysfunction in ion dynamics and signalling, ATP depletion disrupting ATP-dependent processes, ROS generation leading to cellular damage, could all be explained by the effects of TA-289 and equisetin on mitochondria.

A small number of genes involved in cell cycle progression, particularly in the mitotic phase, however a number of $\mathrm{G}_{1}$ cyclins were also identified. As mentioned previously, ROS production, in particular the production of hydroxyl radicals $\cdot \mathrm{OH}$, has been shown to cause a cell cycle block at $\mathrm{G}_{2}$ (Perrone, Tan et al. 2008). Notably, yeast cells also respond to oxidative stress by delaying cell cycle progression (Flattery-O'Brien and Dawes 1998; Chiu, Tactacan et al. 2011), however, the delay would result in delayed entry at $\mathrm{G}_{1}$, whether this is relayed in cells not completing the cell cycle (thus a seemingly $\mathrm{G}_{2}$ block) prior to entry again at $\mathrm{G}_{1}$, it is unclear. Likewise, it could be a combined action, where ROS production induces cell cycle bock and subsequently a delay in entry into $\mathrm{G}_{1}$, which may explain both early and late phase cell cycle-related genes sensitizing to TA-289. 


\subsection{Role of cardiolipin}

Cardiolipin is a phospholipid primarily localized to the mitochondrial inner membrane, constituting about $20 \%$ of total lipid mitochondrial composition (Pangborn 1942; Houtkooper and Vaz 2008). It is an integral component in mitochondrial metabolism, where the lipid has been shown to be required for structural maintenance of the ETC complexes, in particularly complex IV and V which require cardiolipin molecules to maintain full enzymatic function (Eble, Coleman et al. 1990; Robinson, Zborowski et al. 1990). Depletion of cardiolipin significantly reduces complex IV and V activity and mitochondrial protein import, collapsing the mitochondrial transmembrane potential $\left(\Delta \Psi_{\mathrm{m}}\right)$ and disrupting mitochondrial function (Jiang, Ryan et al. 2000). Moreover, oxidation of cardiolipin leads to the formation of hydroperoxides, which can easily break down into the highly reactive hydroxyl radicals (Belikova, Vladimirov et al. 2006).

Considering the pattern of resistance observed across the ETC associated genes tested, it is possible that equisetin and TA-289 target cardiolipin or the biosynthesis of cardiolipin. Binding of cardiolipin may result in many of the observations noted in this study, including 1) ROS generation; as cardiolipin is strongly associated with almost all complexes of the ETC, disrupting cardiolipin will inevitably disrupt the function of the ETC complexes, most likely resulting in induction of ROS from complexes known to be sources of ROS, including complex III ( $b c 1$ complex) and complex I. Aditionally, oxidation of cardiolipin itself will create free radicals; and 2) 
changes in mitochondrial morphology; conformational changes in cardiolipin will result in changes in mitochondrial morphology, and also protons trapped in the lipid (Haines and Dencher 2002) are released, disrupting the proton gradient and potentially disrupting ATP synthesis.

\subsection{Microsomal translocation}

Previous evidence showing inhibition of SecA in vitro (Sugie, al et al. 2002) and translocation of post-translational proteins to the ER (Patham, Duffy et al. 2009) by equisetin, coupled with the ER and endosome/vacuole processes identified in the chemical genomic profile of TA-289 suggests a possible interaction of TA-289 and equisetin at multiple sites, namely the ER and the mitochondrion. The experiments conducted in these chapters do not allow for definite distinction of either a direct site-of-action at the mitochondrion or at the ER, as they are intimately associated organelles. The ER and mitochondria are organelles that co-mediate many key processes, including phospholipid biosynthesis (Gaigg, Simbeni et al. 1995), and ion signalling and homeostasis (Decuypere 2011). Mitochondrial phospholipid synthesis is restricted to the formation of three main lipids: phosphatidylethanolamine, phosphatidylglycerol, and cardiolipin. Microsomal transport of phosphatidylserine from the ER is required in order for mitochondria to synthesise phosphatidylethanolamine (Gaigg, Simbeni et al. 1995). Specialized microsomal fractions have been shown to form a complex with the outer and inner 
mitochondrial membrane to synthesize phosphatidylserine, phosphatidylethanolamine, and phosphatidylcoline (Ardail, Gasnier et al. 1993).

The assays performed in this study identify a probable similar mode-of-action for TA-289 and equisetin, most likely owing to their structural similarity. Our results from the cell cycle analyses, chemical genomic profiling screens, ROS detection and mitochondrial morphology assays indicate that the effects by which TA-289 and equisetin induce cell growth inhibition is dependent on mitochondrial-mediated ROS production as a result of drug-target binding, of which the production presumably stems from complex III of the ETC. Furthermore, resistance to other oxidants displayed by the TA-289 resistant mutant generated in this study indicates the importance of the cell's survival through mediating ROS production and antioxidant capacity in the presence of TA-289. Follow-up testing of genes involved in the ETC and previously described putative gene targets of equisetin have not allowed for strong conclusions on the exact target(s) of TA-289 and equisetin. This, in combination with the reported inhibitory activity of equisetin on the translocation of post-translational protein import to the ER (Patham, Duffy et al. 2009), suggests a possible site-of-action not directly at one of the ETC complexes, but at a site that is tightly associated with the respiratory chain and ER, such as through targeting cardiolipin. Therefore, further studies into the mechanisms of both TA-289 and equisetin will need to be conducted to determine the exact sites- 
of-action of the two compounds, with particular emphasis on the mitochondria and the ER. 


\section{FUTURE DIRECTIONS}

\subsection{Chemical genomic profiling}

The chemical genomic profiling screens of TA-289 performed in this study were conducted under fermenting conditions. As such, genes essential for respiration that may be hypersensitive to TA-289 and equisetin may not be truly represented. To compare the list of genes sensitizing to either compound, a chemical genomic profile screen conducted in non-fermentable carbon sources such as glycerol will allow the identification of genes and processes affected by TA-289 and equisetin that may not have been significantly affected in glucose. Additionally, performing these screens in glycerol may avoid ROS-related hits, and may thus be more directed towards the actual targets of TA-289 and equisetin.

\subsection{Mitochondria}

\subsubsection{Mitochondrial transmembrane potential}

Currently, there is no direct evidence from the studies conducted here that treatment with TA-289 and equisetin ultimately results in the collapsing of the mitochondrial transmembrane potential $(\Delta \Psi \mathrm{m})$; this was not observed in a previous study performed in isolated rat mitochondria (König, Kapus et al. 1993). Probes specifically sensitive to the $\Delta \Psi \mathrm{m}$ can be applied to detect any changes of the $\Delta \Psi \mathrm{m}$ upon TA-289 and equisetin treatment. ATP synthesis is a process that is dependent 
on the mitochondrial proton gradient. As such, ATP depletion can be an indicator of a collapse in the $\Delta \Psi \mathrm{m}$. Measuring intracellular ATP may also provide insight into the effects of TA-289 and equisetin on the energy generating capacity of the mitochondria, as a functioning $\Delta \Psi \mathrm{m}$ is required for ATP-synthesis.

\subsubsection{Mitochondrial deficient mutants}

To determine that TA-289 and equisetin do indeed act on the mitochondria, it can be postulated that mitochondria deficient mutants such as petite yeast mutants will be resistant to TA-289 and equisetin. If mitochondria deficient mutants prove not to be resistant, it could suggest that TA-289 and equisetin either do not act at the mitochondria, or have multiple targets within the cell.

\subsubsection{Cytochrome c release}

The release of cytochrome $c$ from the mitochondria triggers a sequence of proapoptotic effectors leading to cell death. If treatment with TA-289 and equisetin causes the release of cytochrome $c$, it is likely that these compounds kill yeast by apoptosis. However, caspase-independent cell death can also occur. Although the deletion mutant did not display resistance, the detection of the translocation of apoptosis-inducing factor $A I F 1$ from the mitochondria to the nucleus for example, will determine whether induction of cell death (if there is cell death) is caspasedependent or caspase-independent. 


\subsubsection{Cardiolipin}

To determine whether TA-289 and equisetin bind directly to cardiolipin, a cardiolipin rescue assay can be performed by competitive-binding of exogenous cardiolipin with TA-289 and equisetin. Observation of cell growth upon treatment with TA-289 and equisetin in the presence of exogenous cardiolipin, compared to growth inhibition in the absence of exogenous cardiolipin will indicate that both compounds bind cardiolipin. The effects of TA-289 and equisetin on a mutant strain lacking in cardiolipin synthase CRD1 may also be tested to determine the significance of cardiolipin in the ability for both compounds to elicit damage via the production of ROS. In the case where the $\operatorname{crd} 1 \Delta$ mutant displays strong resistance to TA-289 and equisetin suggests that $C R D 1$ may indeed be the target.

\subsubsection{Calcium signalling}

Intracellular calcium $\left(\mathrm{Ca}^{2+}\right)$ homeostasis is part regulated by mitochondria and the ER. Disruption in the dynamics of $\mathrm{Ca}^{2+}$ signalling has been shown to result in a cascade of processes (including mitochondrial and ER dysfunction and ROS generation), leading to cell death (Pozniakovsky, Knorre et al. 2005). Treatment with amiodarone leads to an increase in intracellular $\mathrm{Ca}^{2+}$, causing a loss of mitochondrial membrane potential, ROS production at complex III, and cytochrome $c$ release (Pozniakovsky, Knorre et al. 2005). Equisetin has been previously shown to potently inhibit $\mathrm{Ca}^{2+}$ uptake (König, Kapus et al. 1993). Testing for the increase or 
decrease in cytostolic $\mathrm{Ca}^{2+}$ in TA-289 and equisetin treated cells may infer a similar or dissimilar mechanism to amiodarone.

\subsection{The effect of fermentable and non-fermentable carbon sources}

Further study can be done on the growth inhibitory effects of TA-289 and equisetin in different carbon sources. The ability for cells to grow in the presence of TA-289 and equisetin under one carbon source and when forced to switch metabolism upon exposure to a different carbon source will assess whether this will result in increased sensitivity to TA-289 and equisetin, or resistance. The ability for TA-289 and equisetin to induce ROS in glycerol should also be tested to determine if these effects are more pronounced in glucose (fermentative) or in glycerol (respiratory).

\subsection{Oxidative stress rescue}

Cells have various defence mechanisms which are employed against ROS and oxidative stress. In the case where TA-289 and equisetin cause ROS production leading to oxidative stress in yeast cells, antioxidants and uncouplers may be used to determine if cell growth inhibition by TA-289 and equisetin can be rescued. Glutathione plays an integral role in detoxifying intracellular ROS (Madeo, Frohlich et al. 1999). Addition of glutathione may rescue ROS generated as a result of TA-289 and equisetin. Other antioxidants may be tested, including caffeic acid and ascorbate. Uncouplers such as 2,4-dinitrophenol, which inhibit ATP synthesis 
without affecting the ETC by inhibiting the coupling between electron transfer and phosphorylation reactions, have been shown to prevent mitochondrial-ROS production (Terada 1990; Barros, Bandy et al. 2004). Likewise, co-treatment with known inhibitors of the ETC may alleviate the ROS production caused by TA-289 and equisetin. Moreover, the inhibition of TA-289- and equisetin-induced ROS by ETC inhibitors may give an indication as to where in the ETC TA-289 and equisetin target. For example, the complex III inhibitor antimycin A causes ROS production at complex III (Lai, Zhang et al. 2005). Inhibition at complex IV by KCN does not alleviate the ROS production caused by antimycin A (Turrens 2003). However, inhibition with myxothiazol reduces ROS production caused by antimycin A as myxothiazol prevents the oxidation of Coenzyme Q quinol molecules required to generate ROS (Thierbach and Reichenbach 1981). Moreover, inhibition with the complex I inhibitor rotenone may also reduce ROS induction by antimycin $A$, as electron flow is halted up-stream of complex III (Chen, Vazquez et al. 2003; Li, Ragheb et al. 2003).

\subsection{Testing additional genes}

Validation of select genes significantly sensitizing to TA-289 in the HET screen should be performed to identify any potential gene targets not directly related to the ETC. These include the autophagy-related gene AUT7, which displayed greatest sensitivity to TA-289, and mitochondrial inorganic pyrophosphatase $P P A 2$, which 
has been shown to be required for mitochondrial function. A more comprehensive collection of genes associated with the ETC may also be tested.

\subsection{ER stress and ROS}

The mitochondrion, while it is a major source of ROS, is not the only source. The ER has been implicated in producing $\mathrm{H}_{2} \mathrm{O}_{2}$ during protein folding (Pollard, Travers et al. 1998; Sevier, Qu et al. 2007). The accumulation of mis-folded proteins that lead to ER stress can lead to ROS production within both the ER and the mitochondria (Haynes, Titus et al. 2004). If TA-289 and equisetin disrupt the ER and cause stress which leads to mitochondrial ROS, then it can be implied that both compounds may have multiple targets within the cell, as equisetin was previously shown to act directly at isolated mitochondria (König, Kapus et al. 1993). The source of ROS in this instance can be determined by using mitochondria-deficient petite yeast mutants. ER morphology of petite mutants treated with TA-289 and equisetin can be assessed using fluorescent protein probes visualized using a high-throughput confocal microscope.

\subsection{Phosphatidylserine import}

Phosphatidylserine is transported by specialized subfractions of the ER known as microsomes into the mitochondria in order for the synthesis of phosphatidylethanolamine, one of the key phospholipids synthesized at the 
mitochondria. The conversion of phosphatidylserine into phosphatidylethanolamine indicates the level of phosphatidylserine import into the mitochondria, and may serve to suggest that if TA-289 and equisetin target microsomal translocation (which may explain the previously described inhibitory activities of equisetin on transport by translocon (Patham, Duffy et al. 2009)), there will be reduced conversion of phosphatidylserine to phosphatidylethanolamine. 


\section{APPENDIX 1}

\subsection{Biologically active compounds}

Of the 88 compounds screened initially, 22 small molecules were identified as bioactive in wt (BY4742) cells (> 70\% growth inhibition compared to DMSO carrier controls). Ten additional "hits" were observed in the pdr1 $\operatorname{mutant}$ strain (highlighted in blue) which, did not appear when screened against the wt strain. Commercial names of the small molecules were provided by Dr. Stephen Bloor, and a brief description of each compound is detailed; tetramic-acid 289 (TA-289) and equisetin (highlighted in pink) were chosen to further target identification studies. A hit of "1" indicates that the compound is biologically active in the described yeast strain. A hit of "0" indicates that the compound displayed no biological activity in the described yeast strain.

\begin{tabular}{|l|l|l|l|}
\hline $\begin{array}{l}\text { Wt } \\
\text { "hit" }\end{array}$ & $\begin{array}{l}\text { pdr1s } \\
\text { "hit" }\end{array}$ & $\begin{array}{l}\text { Compound } \\
\text { name }\end{array}$ & Description \\
\hline 1 & 1 & 402 Pyrone & Novel, uncharacterized. \\
\hline 1 & 1 & citrinin & $\begin{array}{l}\text { Induces mitochondrial permeability } \\
\text { transition and inhibits microtubule } \\
\text { polymerization (Chan 2007; Iwahashi, } \\
\text { Kitagawa et al. 2007). }\end{array}$ \\
\hline 1 & 1 & $\begin{array}{l}\text { tetramic acid- } \\
289 \text { (TA-289) }\end{array}$ & Novel, uncharacterized. \\
\hline 1 & 1 & traversianal & $\begin{array}{l}\text { Causes cellular leakage, shown to lyse } \\
\text { human red blood cells (Stoessl, Cole et al. } \\
\text { 1989). }\end{array}$ \\
\hline 1 & 1 & sterigmatocystin & $\begin{array}{l}\text { DNA synthesis inhibitor. Precursor of } \\
\text { aflatoxin, a known carcinogen (Cary, }\end{array}$ \\
\hline
\end{tabular}




\begin{tabular}{|c|c|c|c|}
\hline & & & Ehrlich et al. 2009). \\
\hline 1 & 1 & zaragozic acid & $\begin{array}{l}\text { Inhibits both fungal and mammalian } \\
\text { squalene synthases. Stimulates alpha- } \\
\text { secretase activity (Kojro, Fuger et al. } \\
\text { 2010). }\end{array}$ \\
\hline 1 & 1 & fumonisin B2 & $\begin{array}{l}\text { Analogue of fumonisin B1. Inhibits } \\
\text { ceramide synthase and induces apoptosis } \\
\text { (Desai, Sullards et al. 2002). }\end{array}$ \\
\hline 1 & 1 & pamamycin & $\begin{array}{l}\text { Alters membrane-associated cellular } \\
\text { functions, inhibits uptake of nucleosides } \\
\text { (Chou and Pogell 1981; Chou and Pogell } \\
\text { 1981). }\end{array}$ \\
\hline 1 & 1 & phoslactomycin & $\begin{array}{l}\text { Inhibits phosphotase 2A (Usui, Marriott et } \\
\text { al. 1999). }\end{array}$ \\
\hline 1 & 1 & actinomycin & $\begin{array}{l}\text { DNA synthesis inhibitor (Flamee 1985; } \\
\text { Sobell 1985). }\end{array}$ \\
\hline 1 & 1 & talaro TS1-14 & Novel, uncharacterized. \\
\hline 1 & 1 & candin & $\begin{array}{l}\text { Inhibits beta-1,3-glucan synthase } \\
\text { (Denning 2003). }\end{array}$ \\
\hline 1 & 1 & $\begin{array}{c}\mathrm{N}-\mathrm{OH} \\
\text { apiosporamide }\end{array}$ & $\begin{array}{l}\text { Antifungal activity (Alfatafta, Gloer et al. } \\
\text { 1994). }\end{array}$ \\
\hline 1 & 1 & reveromycin & $\begin{array}{l}\text { Inhibits isoleucyl-tRNA synthetase } \\
\text { activity. Induces apoptosis (Shimizu, Usui } \\
\text { et al. 2002; Woo 2007). }\end{array}$ \\
\hline 1 & 1 & avenociolide & $\begin{array}{l}\text { Uncharacterized (Chen, Narkunan et al. } \\
\text { 1999). }\end{array}$ \\
\hline 1 & 1 & arthrinic acid & Novel, Antifungal activity (Bloor 2008). \\
\hline 1 & 1 & pycnidione & Novel, uncharacterized. \\
\hline 1 & 1 & fumifungin & $\begin{array}{l}\text { Antifungal activity (Mukhopadhyay, Roy } \\
\text { et al. 1987). }\end{array}$ \\
\hline 1 & 1 & equisetin & $\begin{array}{l}\text { Antibacterial activity. Inhibitory activity } \\
\text { against human immunodeficiency virus } \\
\text { type } 1 \text { (HIV-1) integrase (Vesonder, Tjarks } \\
\text { et al. 1979; Hazuda, Blau et al. 1999). }\end{array}$ \\
\hline
\end{tabular}




\begin{tabular}{|c|c|c|c|}
\hline 1 & 1 & borrelidin & $\begin{array}{l}\text { Inhibits threonyl tRNA synthase and } \\
\text { activates Caspase-8/3 pathway (Saisivam, } \\
\text { Bhikshapathi et al. 2008; Bhikshapathi, } \\
\text { Kumar et al. 2010). }\end{array}$ \\
\hline 1 & 1 & niphimycin A & $\begin{array}{l}\text { Antifungal activity Structurally related to } \\
\text { amphotericin B, a known ergosterol } \\
\text { binder (Usuki, Matsumoto et al. 2006; } \\
\text { Ogita, Matsumoto et al. 2007). }\end{array}$ \\
\hline 1 & 1 & $\begin{array}{l}\text { viridicatum } \\
\text { toxin }\end{array}$ & Antibiotic activity (Zheng, Yu et al. 2008). \\
\hline 0 & 1 & angucyclinone & $\begin{array}{l}\text { Antibiotic activity (Schimana, Fiedler et } \\
\text { al. 2000). }\end{array}$ \\
\hline 0 & 1 & calbistrin & $\begin{array}{l}\text { Antifungal activity (Tatsuta, Itoh et al. } \\
\text { 1997; Stewart, Capon et al. 2005). }\end{array}$ \\
\hline 0 & 1 & cladosporin & $\begin{array}{l}\text { Inhibits uptake of nucleosides (Scott, } \\
\text { Vanwalbe.W et al. 1971; Anke 1979). }\end{array}$ \\
\hline 0 & 1 & paxisterol & $\begin{array}{l}\text { Antifungal activity (Nakano, Hara et al. } \\
\text { 1988). }\end{array}$ \\
\hline 0 & 1 & epoxyphomin & Novel, uncharacterized. \\
\hline 0 & 1 & cytochalasin F & $\begin{array}{l}\text { Actin depolymerizer (Binder and Tamm } \\
\text { 1973). }\end{array}$ \\
\hline 0 & 1 & S39163F1 & Novel, uncharacterized. \\
\hline 0 & 1 & radicicol & $\begin{array}{l}\text { Antibiotic activity. Binds to N-terminal } \\
\text { domain of heat shock protein } 90 \text { (Hsp90) } \\
\text { (Nozawa and Nakajima 1979; Schulte, } \\
\text { Akinaga et al. 1998). }\end{array}$ \\
\hline 0 & 1 & kampanol A & $\begin{array}{l}\text { Farnesyl-protein transferase (FPTase) } \\
\text { inhibitor (Singh, Zink et al. 1998). }\end{array}$ \\
\hline 0 & 1 & calbistrin & $\begin{array}{l}\text { Antifungal activity (Tatsuta, Itoh et al. } \\
\text { 1997; Stewart, Capon et al. 2005). }\end{array}$ \\
\hline
\end{tabular}




\subsection{Liquid-dose response assays of TA-289 and equisetin in varying $\mathrm{pH}$ conditions}

The graphs below display the biological activity of TA-289 and equisetin against wt

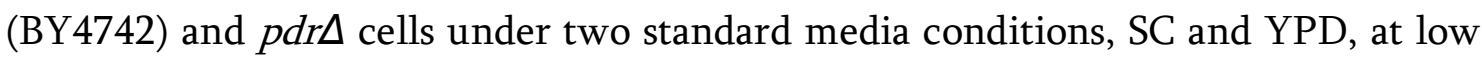
and high $\mathrm{pH}(4.0$ and 7.0-8.0).
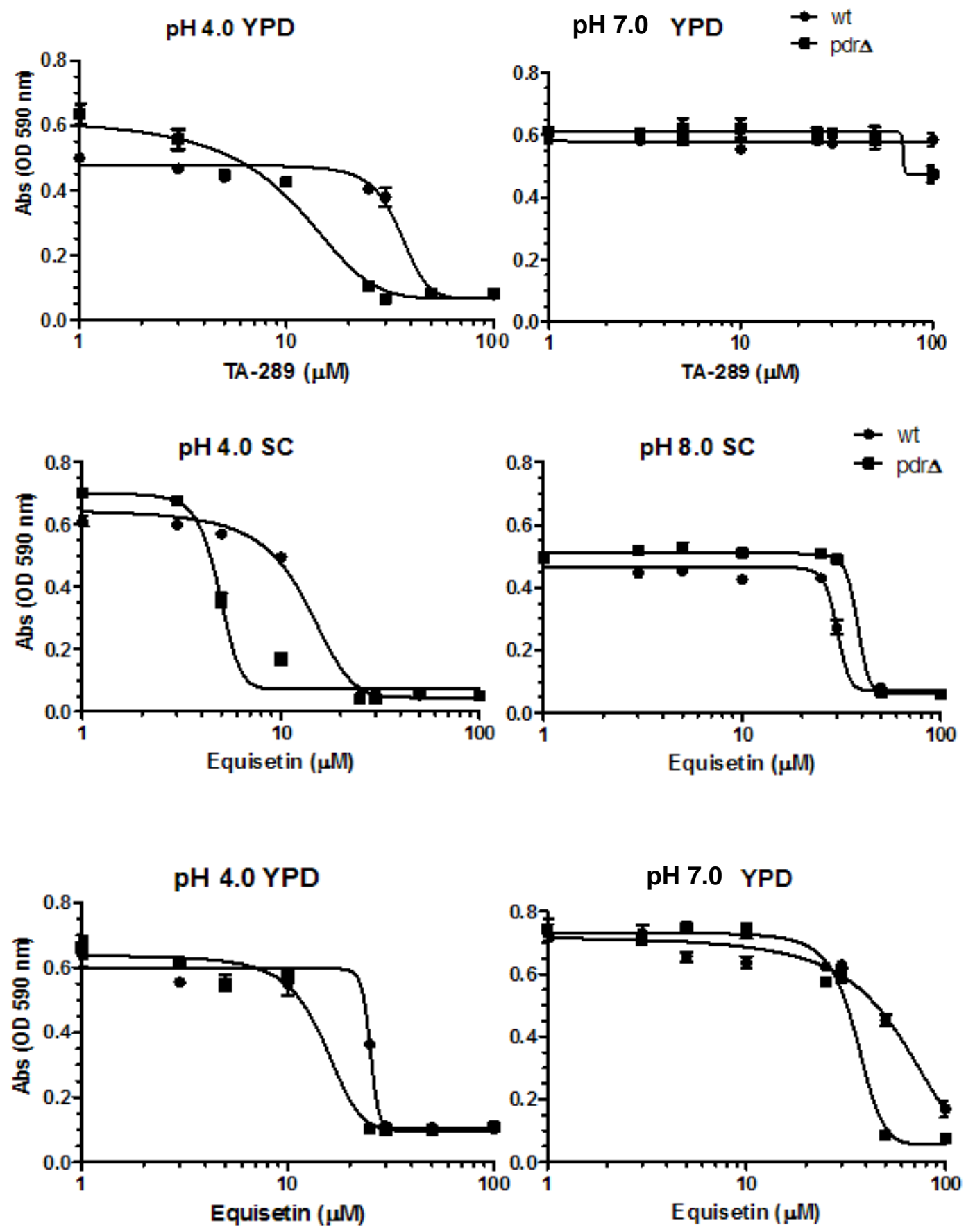


\subsection{Cell morphology of TA-289 cells}

The photographs below show the small-size phenotype displayed by $p d r \Delta$ cells after

$18 \mathrm{~h}$ treatment with TA-289 at $30 \mu \mathrm{M}$ and $100 \mu \mathrm{M}$ compared to untreated controls.

\section{Untreated control:}

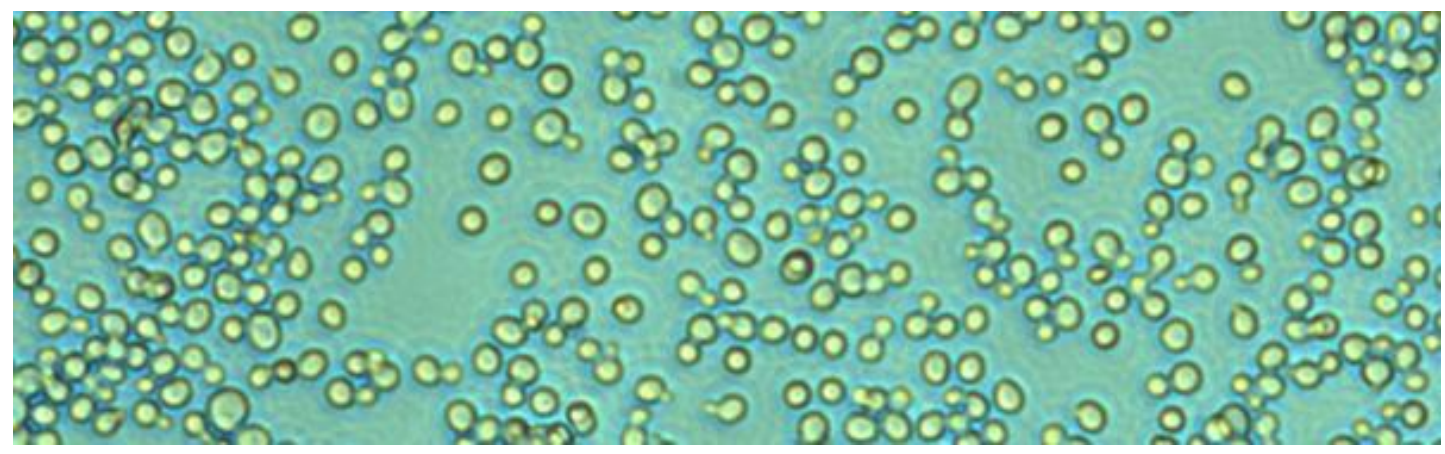

$30 \mu \mathrm{M}$ TA-289:

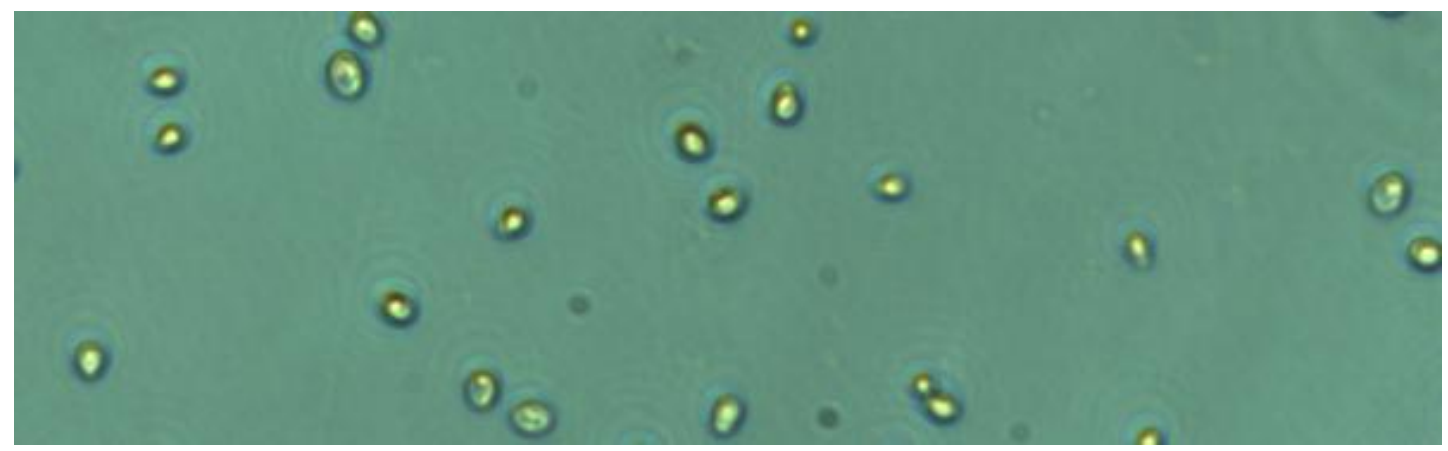

$100 \mu \mathrm{M}$ TA-289:

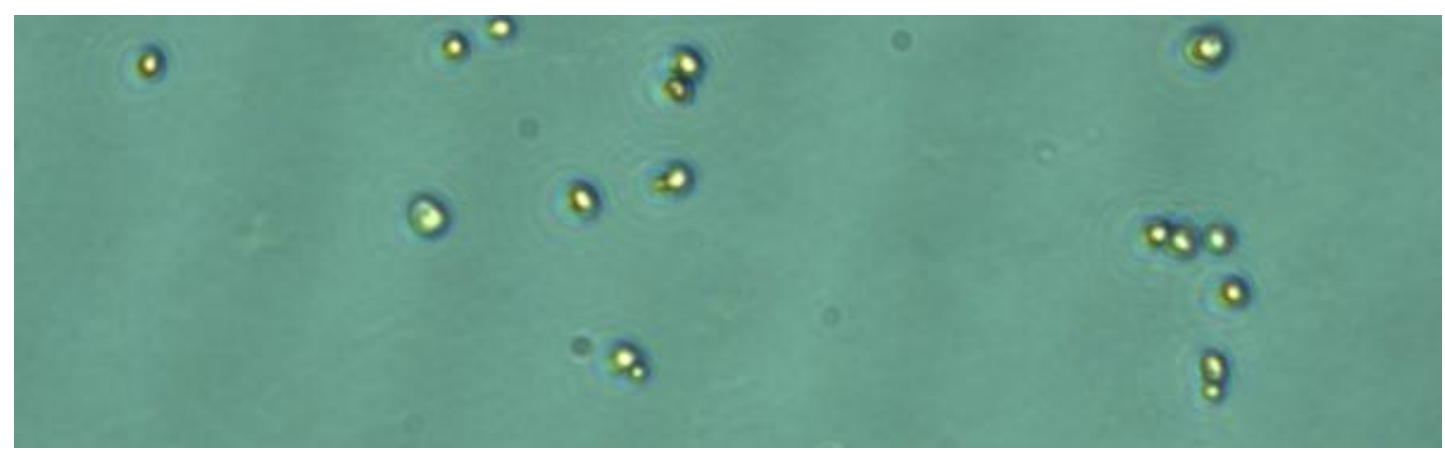




\subsection{Cell cycle analysis by flow cytometry: Time-course histograms}

The figures below show time-course cell cycle analysis of wt (BY4742) and pdr $\Delta$ cells treated with TA-289 and equisetin. The following histograms show time-course of equisetin-treated cells, and TA-289-treated wt cells, and the corresponding wt control. Reported time-course histograms for $p d r \Delta$ cells treated with TA-289 and the corresponding $p d r \Delta$ control have been previously described in chapter two results figure 2.8. $p d r \Delta$ cells treated with equisetin (top right) did not recover from treatment with equisetin after 90 mins of washing. In both wt cell samples treated with TA-289 (bottom left) and equisetin (bottom right), cells recovered slowly after washing.
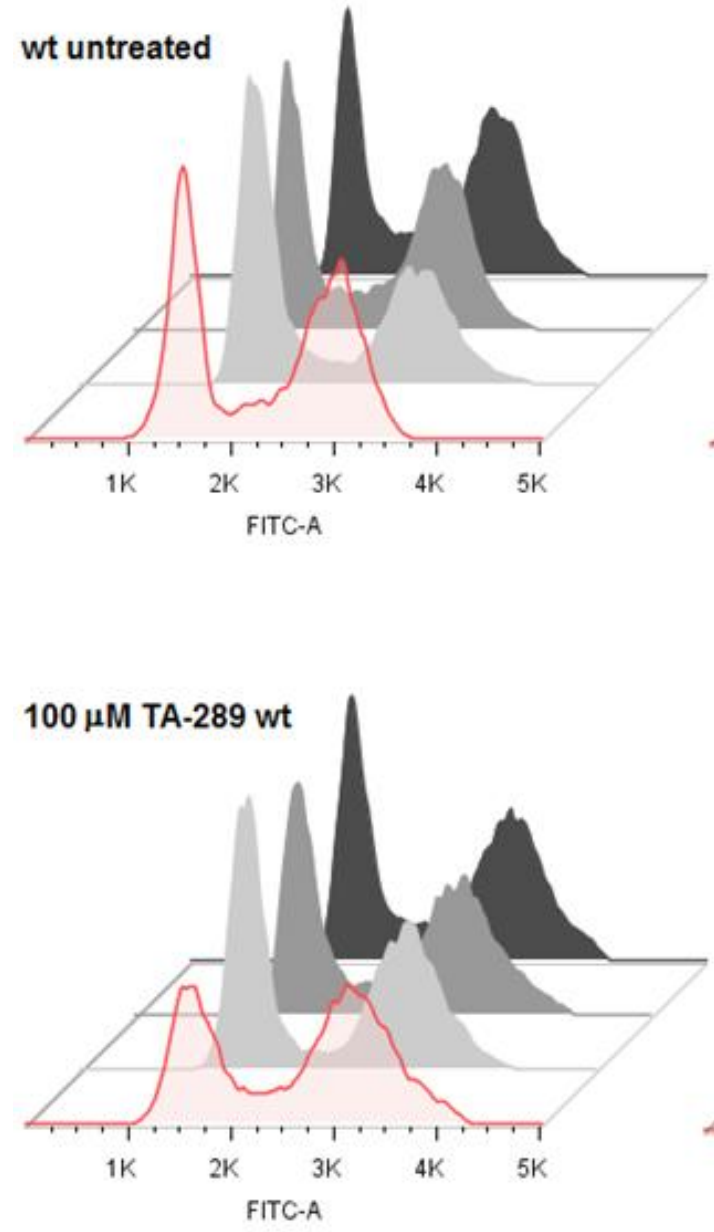

$100 \mu \mathrm{M}$ Equisetin (pdr $\Delta$ )

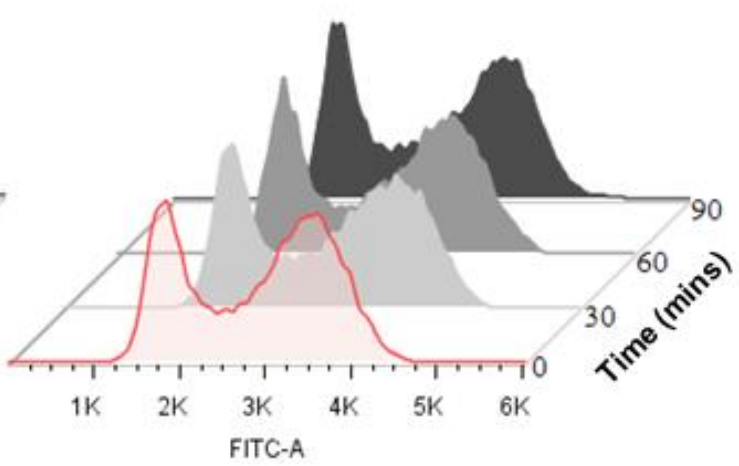

$100 \mu \mathrm{M}$ Equisetin (wt)

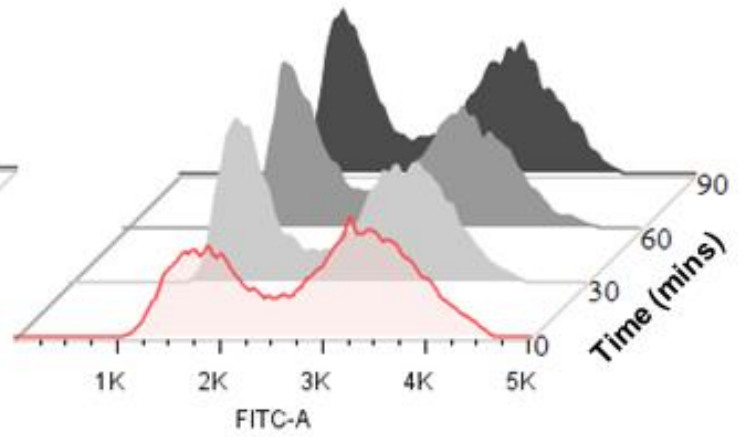




\subsection{Cluster analysis}

Full clustergram with both TA-289 HOM screens highlighted in blue.

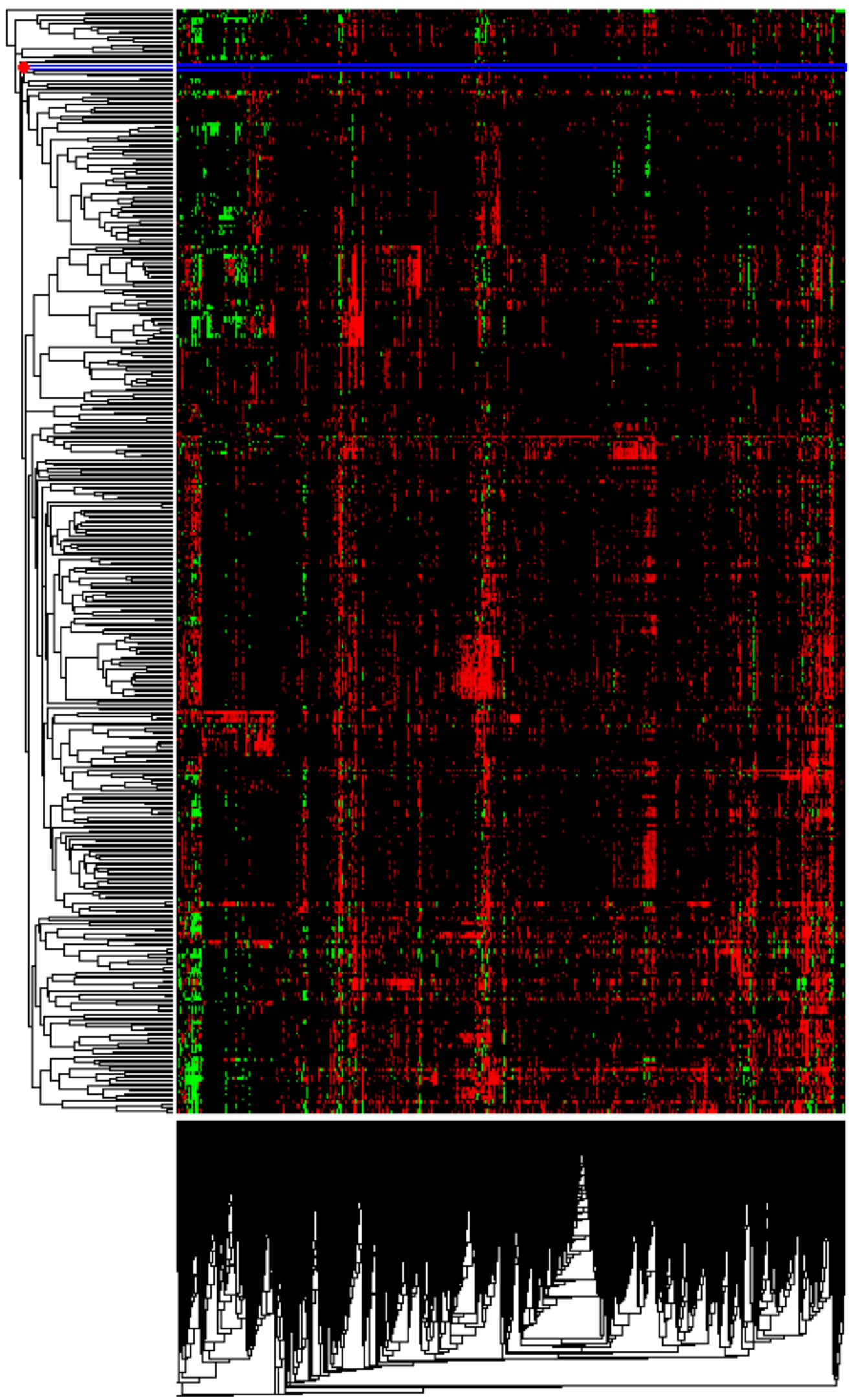




\subsection{TA-289 HOM screen overlaps with L-Dopa and MPP+}

The Venn diagram below illustrates the number of gene overlaps identified between the two combined HOM screens for TA-289 and L-Dopa and MPP+.

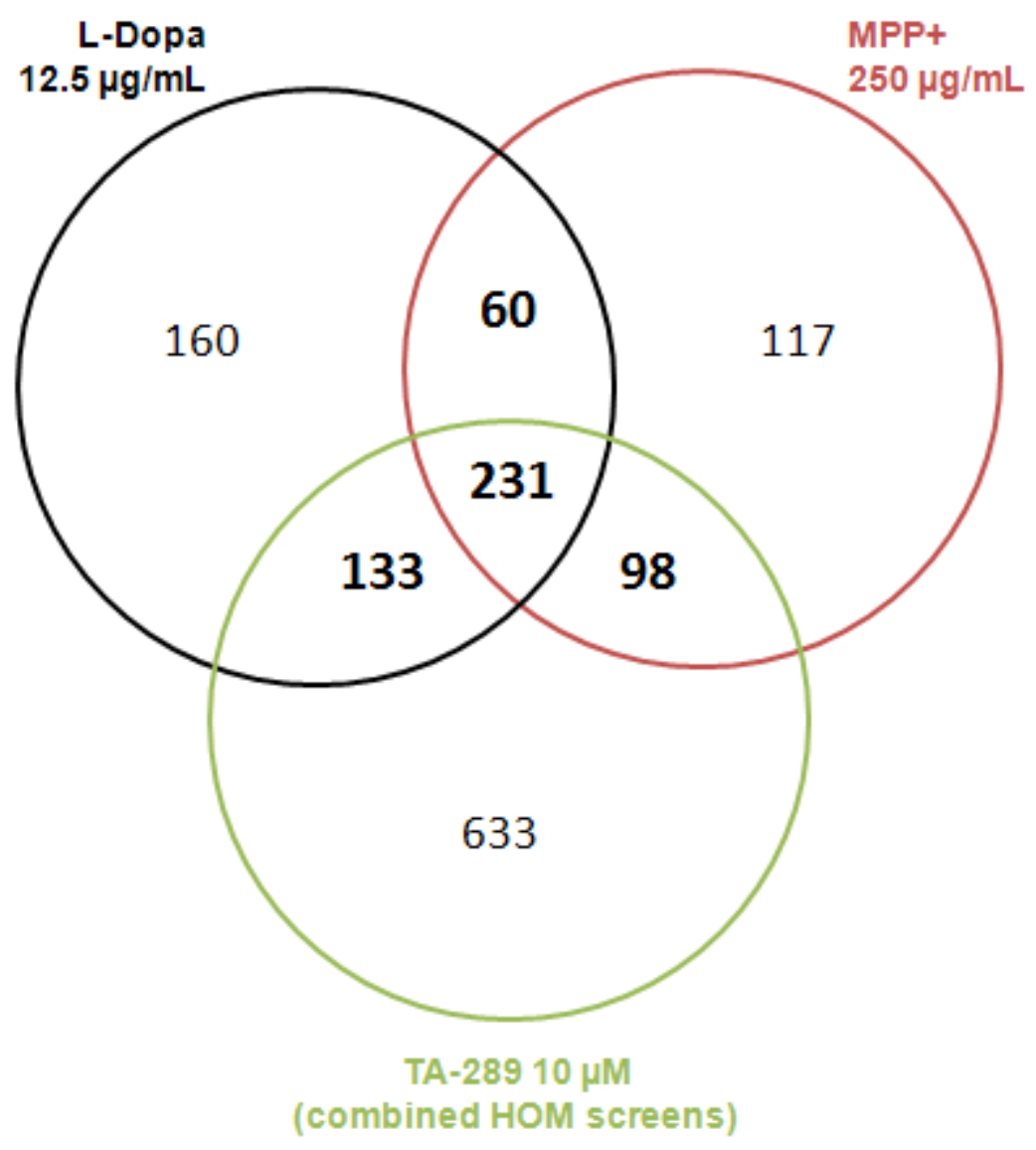


The table below details the gene hits of L-Dopa and MPP+ (Hillenmeyer 2008) compared to the two HOM screens of TA-289. The values indicate the $\log _{2}$ ratios of treated/untreated control, and a threshold was set where values $>0.5$ were deemed as a sensitive hit and values $<-1$ were deemed as resistant. Highlighted in blue are the genes localised to the mitochondrion and the ER (41 genes), and in pink are genes involved in DNA damage and repair and/or oxidative stress (16 genes) that overlap between the TA-289 HOM screens and the L-Dopa and MPP+ screens.

KEY: Golgi $=$ Golgi apparatus, Mito $=$ Mitochondrion, Cyt $=$ Cytoplasm, ER = Endoplasmic reticulum, Nuc $=$ Nucleus, $\mathrm{Vac}=$ Vacuole, Mem $=$ Plasma membrane, Perox $=$ Peroxisome, Endo $=$ Endosome

\begin{tabular}{|c|c|c|c|c|c|}
\hline ORF & $\begin{array}{l}\text { L-Dopa } \\
12.5 \mu \mathrm{g} / \mathrm{mL}\end{array}$ & $\begin{array}{l}\mathrm{MPP}+ \\
250 \mu \mathrm{g} / \mathrm{mL}\end{array}$ & $\begin{array}{l}\text { TA-289 } \\
\text { HOM1 }\end{array}$ & $\begin{array}{l}\text { TA-289 } \\
\text { HOM2 }\end{array}$ & $\begin{array}{l}\text { Cellular } \\
\text { Component }\end{array}$ \\
\hline YAL026C & 0.73 & 0.00 & 0.00 & 0.59 & Golgi \\
\hline YAR014C & 0.84 & 0.00 & 0.00 & 0.56 & Bud neck \\
\hline YBL022C & 3.75 & 0.00 & 0.00 & 0.90 & Mito \\
\hline YBL047C & 0.00 & 2.27 & 0.00 & 0.72 & Bud neck \\
\hline YBL051C & 2.57 & 2.57 & 0.57 & 0.00 & Cyt \\
\hline YBL065W & 0.52 & 0.81 & 0.00 & 3.56 & N/A \\
\hline YBL081W & 0.00 & 0.64 & 0.00 & 1.10 & N/A \\
\hline YBL101C & 0.00 & 1.26 & 0.00 & 0.55 & Cyt \\
\hline YBR003W & 0.55 & 0.00 & 0.00 & 0.58 & Mito \\
\hline YBR006W & 0.00 & 1.58 & 0.00 & 0.65 & Cyt \\
\hline YBR015C & 2.05 & 0.00 & 0.00 & 0.62 & Golgi \\
\hline YBR036C & 0.00 & 1.13 & 0.00 & 2.70 & ER \\
\hline YBR075W & 0.62 & 1.20 & 0.00 & 0.52 & N/A \\
\hline YBR098W & 1.00 & 0.00 & 0.00 & 0.00 & Nuc \\
\hline YBR134W & 0.00 & 0.71 & 0.00 & 0.56 & N/A \\
\hline YBR173C & 1.42 & 1.51 & 0.00 & -1.53 & Cyt, Nuc \\
\hline YBR174C & 1.22 & 0.00 & 0.66 & 0.00 & N/A \\
\hline YBR175W & 0.00 & 0.63 & 0.55 & 0.00 & Nuc \\
\hline YBR213W & 0.00 & 0.82 & 0.00 & -1.35 & N/A \\
\hline YBR244W & 0.53 & 0.00 & 0.00 & 0.00 & Cyt, Nuc \\
\hline YBR278W & 0.00 & 1.81 & 0.00 & 1.23 & Nuc \\
\hline
\end{tabular}




\begin{tabular}{|c|c|c|c|c|c|}
\hline YBR284W & 0.00 & 1.48 & 0.54 & 0.00 & N/A \\
\hline YBR295W & 0.00 & 1.54 & 0.00 & 0.62 & Mem \\
\hline YBR298C & 0.00 & 4.05 & 0.00 & 1.61 & Mem \\
\hline YBR300C & 0.00 & 1.36 & 0.00 & 0.91 & N/A \\
\hline YCL005W & 1.24 & 0.00 & 1.36 & 0.55 & Mito \\
\hline YCL008C & 1.34 & 4.77 & 0.00 & 0.80 & Endo \\
\hline YCL011C & 0.81 & 0.00 & 0.60 & 0.72 & Nuc \\
\hline YCL032W & 1.22 & 0.00 & 0.51 & 0.00 & Cyt \\
\hline YCL037C & 3.13 & 0.00 & 0.00 & 0.00 & Polysome \\
\hline YCL061C & 0.91 & 0.00 & 0.61 & 0.73 & Nuc \\
\hline YCR008W & 0.00 & 0.91 & 0.00 & 0.62 & N/A \\
\hline YCR010C & 1.41 & 0.00 & 0.00 & 0.62 & Mito \\
\hline YCR024C & 0.00 & 3.12 & 0.00 & -1.54 & Mito \\
\hline YCR025C & 2.34 & 2.47 & 0.00 & 0.54 & N/A \\
\hline YCR049C & 0.00 & 1.29 & 0.56 & 0.00 & N/A \\
\hline YCR061W & 2.93 & 3.80 & 0.84 & 0.70 & Cyt \\
\hline YCR065W & 2.61 & 0.00 & 0.00 & 0.60 & Cyt, Nuc \\
\hline YCR076C & 0.88 & 0.00 & 0.56 & 0.00 & N/A \\
\hline YCR087W & 3.84 & 1.01 & 0.00 & 0.66 & N/A \\
\hline YCR095C & 0.00 & 1.96 & 0.00 & 0.67 & Cyt \\
\hline YDL001W & 1.55 & 0.65 & 0.00 & 0.75 & Cyt \\
\hline YDL062W & 0.52 & 0.00 & 0.00 & 0.51 & N/A \\
\hline YDL074C & 0.66 & 0.80 & 0.00 & 0.56 & Nuc \\
\hline YDL100C & 0.00 & 0.96 & 0.00 & 0.53 & ER \\
\hline YDL133W & 0.00 & 1.34 & 0.00 & 0.91 & Mem \\
\hline YDL162C & 3.49 & 0.00 & 0.55 & 0.00 & N/A \\
\hline YDL241W & 4.10 & 0.00 & 0.62 & 0.59 & N/A \\
\hline YDR034C & 0.95 & 0.00 & -1.07 & 0.00 & Nuc \\
\hline YDR076W & 2.75 & 1.16 & 0.00 & 0.00 & Nuc \\
\hline YDR105C & 0.87 & 0.64 & 0.00 & -1.10 & Vac \\
\hline YDR126W & 0.77 & 0.00 & 0.00 & 0.75 & ER \\
\hline YDR149C & 3.31 & 0.74 & 0.00 & 0.78 & N/A \\
\hline YDR162C & 2.11 & 0.00 & 0.00 & 0.00 & Cyt, Nuc \\
\hline YDR270W & 0.83 & 1.21 & 0.00 & 0.51 & Golgi \\
\hline YDR272W & 0.78 & 0.56 & 0.00 & 0.97 & Cyt \\
\hline YDR293C & 2.62 & 0.00 & 0.00 & 1.27 & Bud neck \\
\hline YDR310C & 0.00 & 7.61 & 0.53 & 0.00 & Nuc \\
\hline YDR335W & 0.57 & 0.00 & 0.00 & 0.68 & Cyt, Nuc \\
\hline YDR438W & 1.87 & 0.00 & 0.68 & 0.00 & Mito \\
\hline YDR441C & 0.72 & 0.00 & 0.51 & 2.08 & Cyt \\
\hline YDR452W & 0.00 & 0.69 & 0.00 & 1.02 & Vac \\
\hline YDR463W & 0.62 & 0.54 & 0.00 & 0.73 & Cyt, Nuc \\
\hline YDR484W & 3.62 & 0.76 & 0.00 & 0.80 & Golgi \\
\hline YDR520C & 0.94 & 1.35 & 0.00 & 1.19 & Cyt, Nuc \\
\hline YDR522C & 2.12 & 0.00 & 0.53 & 0.00 & Mem \\
\hline YEL011W & 0.59 & 0.00 & 0.00 & 0.50 & Cyt \\
\hline
\end{tabular}




\begin{tabular}{|c|c|c|c|c|c|}
\hline YEL015W & 2.11 & 0.00 & 0.00 & 0.96 & Cyt \\
\hline YEL031W & 0.00 & 4.45 & 0.00 & -1.45 & ER \\
\hline YER034W & 0.57 & 0.00 & 0.00 & 0.59 & N/A \\
\hline YER078C & 1.42 & 1.94 & 0.00 & 2.30 & Mito \\
\hline YER114C & 4.92 & 0.00 & 0.00 & 0.68 & Bud neck \\
\hline YER169W & 0.00 & 1.14 & 0.56 & 0.00 & Nuc \\
\hline YFL025C & 3.24 & 0.00 & 0.80 & 0.61 & ER \\
\hline YFR007W & 0.00 & 0.68 & 0.61 & 0.70 & N/A \\
\hline YFR040W & 2.82 & 0.00 & 0.00 & 0.78 & Cyt \\
\hline YGL054C & 1.01 & 0.00 & 0.00 & 0.75 & ER \\
\hline YGL066W & 2.51 & 0.00 & 0.00 & 1.55 & Cyt, Nuc \\
\hline YGL071W & 5.16 & 1.44 & 0.00 & 2.09 & Cyt, Nuc \\
\hline YGL086W & 1.02 & 0.00 & 0.00 & 0.89 & $\mathrm{Nuc}$ \\
\hline YGL087C & 0.00 & 1.71 & 0.00 & 0.74 & Cyt, Nuc \\
\hline YGL115W & 1.92 & 0.00 & 0.76 & 0.00 & Cyt, Nuc \\
\hline YGL147C & 0.00 & 2.58 & 0.58 & 0.73 & Cyt, Nuc \\
\hline YGL148W & 0.61 & 0.00 & 0.00 & 0.69 & Cyt \\
\hline YGL198W & 0.63 & 0.00 & 0.66 & 0.00 & Golgi \\
\hline YGL212W & 0.71 & 0.00 & 0.00 & -1.24 & Vac \\
\hline YGL229C & 0.64 & 0.73 & 0.00 & 1.66 & Mito \\
\hline YGL236C & 0.51 & 0.00 & 0.59 & 0.00 & Mito \\
\hline YGL249W & 0.59 & 0.00 & 0.00 & 0.71 & Nuc \\
\hline YGL254W & 0.98 & 0.00 & 0.77 & 0.82 & Nuc \\
\hline YGR034W & 0.65 & 0.00 & 0.00 & -1.05 & Cyt, Nuc \\
\hline YGR106C & 0.53 & 0.00 & 0.00 & 0.57 & ER \\
\hline YGR135W & 1.60 & 0.87 & 0.00 & -0.50 & Mito, Nuc \\
\hline YGR166W & 0.57 & 0.00 & 0.00 & 0.60 & Golgi \\
\hline YGR184C & 0.00 & 0.52 & 0.00 & 0.73 & Cyt \\
\hline YGR201C & 0.59 & 0.55 & 0.00 & 0.00 & N/A \\
\hline YGR210C & 0.96 & 0.74 & 0.00 & 0.73 & Cyt \\
\hline YHL005C & 1.92 & 1.67 & 0.00 & 0.00 & N/A \\
\hline YHL026C & 2.08 & 0.00 & 0.00 & 0.56 & N/A \\
\hline YHR039C & 1.61 & 1.03 & 0.00 & 1.20 & ER \\
\hline YHR046C & 2.07 & 0.00 & 0.00 & 1.02 & Cyt/ Nuc \\
\hline YHR051W & 1.05 & 0.71 & 0.00 & 0.71 & Mito \\
\hline YHR129C & 5.07 & 0.00 & 0.00 & 1.01 & Cyt \\
\hline YHR134W & 2.13 & 0.00 & 0.00 & 0.00 & Nuc \\
\hline YHR154W & 1.41 & 1.02 & 0.00 & 1.13 & Nuc \\
\hline YHR179W & 5.89 & 0.00 & 0.57 & 0.00 & Mito \\
\hline YIL012W & 5.63 & 0.00 & 0.00 & 0.59 & N/A \\
\hline YIL029C & 0.55 & 2.47 & 0.56 & 0.00 & N/A \\
\hline YIL034C & 1.20 & 0.00 & 0.00 & -1.11 & Bud neck \\
\hline YIL035C & 2.72 & 1.63 & 0.00 & 1.16 & Cyt, Nuc \\
\hline YIL085C & 0.00 & 0.75 & 0.54 & 0.00 & Golgi \\
\hline YIL097W & 0.00 & 0.77 & 0.58 & 0.00 & Cyt, Nuc \\
\hline YIL103W & 2.36 & 1.56 & 0.00 & 0.78 & Cyt, Nuc \\
\hline
\end{tabular}




\begin{tabular}{|c|c|c|c|c|c|}
\hline YIL105C & 0.88 & 0.00 & 0.00 & 0.58 & Mem \\
\hline YIL132C & 0.84 & 0.00 & 0.00 & 0.00 & Cyt, Nuc \\
\hline YIL139C & 0.76 & 1.59 & 0.00 & 0.00 & Mito \\
\hline YIL157C & 4.03 & 1.53 & 0.00 & 1.83 & Mito \\
\hline YIL170W & 0.66 & 0.00 & 0.53 & 0.00 & N/A \\
\hline YIR037W & 0.50 & 1.00 & 0.00 & 0.64 & N/A \\
\hline YJL047C & 2.92 & 0.00 & 0.00 & 0.00 & Cyt, Nuc \\
\hline YJL092W & 1.83 & 3.08 & 0.00 & 0.00 & Nuc \\
\hline YJL126W & 0.00 & 1.05 & 0.00 & 0.65 & N/A \\
\hline YJL141C & 5.14 & 0.00 & 0.00 & 0.79 & Cyt, Nuc \\
\hline YJL145W & 0.00 & 0.74 & -1.07 & 0.00 & ER \\
\hline YJL154C & 0.79 & 0.00 & 0.65 & 0.75 & Endo \\
\hline YJL162C & 0.00 & 1.43 & 0.63 & 0.00 & N/A \\
\hline YJL192C & 5.50 & 0.00 & 0.00 & 0.63 & ER \\
\hline YJL204C & 0.61 & 0.00 & 0.00 & 0.00 & Golgi, Endo \\
\hline YJL217W & 1.41 & 0.00 & 0.72 & 0.56 & Cyt \\
\hline YJR010C-A & 0.00 & 0.80 & 0.00 & 0.56 & ER \\
\hline YJR043C & 2.37 & 0.00 & 0.00 & 0.50 & Nuc \\
\hline YJR102C & 3.45 & 4.31 & 0.00 & 1.90 & Endo, Vac \\
\hline YJR128W & 0.00 & 0.61 & 0.00 & 0.59 & N/A \\
\hline YJR140C & 0.00 & 1.31 & 0.00 & 0.57 & Nuc \\
\hline YKL037W & 0.00 & 1.11 & 0.00 & 1.47 & Mito \\
\hline YKL136W & 1.18 & 0.00 & 0.63 & 0.00 & N/A \\
\hline YKL149C & 4.24 & 0.00 & 0.00 & -1.33 & Nuc \\
\hline YKL150W & 0.67 & 1.46 & 0.00 & 0.63 & Mito \\
\hline YKL197C & 0.00 & 3.50 & 0.00 & -1.14 & Perox \\
\hline YKR017C & 0.70 & 0.00 & 0.00 & 0.65 & N/A \\
\hline \multicolumn{6}{|l|}{ YKR035W- } \\
\hline A & 1.30 & 0.00 & 0.00 & 0.56 & Cyt, Endo \\
\hline YKR074W & 3.38 & 2.34 & 0.00 & 0.54 & Cyt \\
\hline YLL014W & 1.03 & 0.00 & 0.00 & 0.58 & ER \\
\hline YLR025W & 0.00 & 5.10 & 0.00 & 1.53 & Endo \\
\hline YLR027C & 4.88 & 0.00 & 0.00 & 1.10 & Perox \\
\hline YLR032W & 3.21 & 0.73 & 0.63 & 0.00 & Nuc \\
\hline YLR049C & 0.56 & 0.66 & 0.00 & 0.83 & N/A \\
\hline YLR125W & 0.81 & 0.00 & 0.72 & 0.00 & N/A \\
\hline YLR386W & 0.51 & 0.00 & 0.00 & -1.38 & Vac \\
\hline YLR402W & 3.71 & 0.00 & 0.00 & 0.51 & N/A \\
\hline YLR417W & 0.54 & 5.33 & 0.00 & 1.12 & Endo \\
\hline YLR418C & 0.00 & 1.92 & 0.00 & 0.55 & Nuc \\
\hline YML013W & 4.37 & 0.00 & 0.00 & 0.70 & ER \\
\hline YML071C & 0.60 & 0.00 & 0.61 & 0.73 & Golgi \\
\hline YML097C & 0.57 & 0.00 & 1.15 & 0.00 & Vac \\
\hline YML121W & 0.87 & 0.00 & 0.00 & 1.04 & Cyt, Endo \\
\hline YMR077C & 0.87 & 4.30 & 0.00 & 1.54 & Endo \\
\hline YMR098C & 1.31 & 1.26 & 0.00 & 1.53 & Mito \\
\hline
\end{tabular}




\begin{tabular}{|c|c|c|c|c|c|}
\hline YMR115W & 0.53 & 0.00 & 0.00 & -1.00 & Mito \\
\hline YMR121C & 0.00 & 1.16 & 0.00 & 0.69 & Cyt \\
\hline YMR152W & 1.36 & 0.00 & 0.00 & 1.09 & Mito, Cyt \\
\hline YMR179W & 1.50 & 0.66 & 0.00 & 0.93 & Nuc \\
\hline YMR263W & 1.04 & 3.85 & 0.00 & 0.66 & Nuc \\
\hline YMR272C & 0.00 & 1.15 & 0.00 & 1.13 & ER \\
\hline \multicolumn{6}{|l|}{ YMR304C- } \\
\hline $\mathrm{A}$ & 0.80 & 0.51 & 0.00 & 0.50 & N/A \\
\hline YNL041C & 5.73 & 0.00 & 0.67 & 0.00 & Golgi \\
\hline YNL071W & 2.20 & 4.27 & 0.00 & 0.55 & Mito \\
\hline YNL077W & 0.00 & 2.41 & 0.00 & 0.56 & Mito \\
\hline YNL084C & 3.02 & 0.00 & 0.00 & 1.21 & Bud neck \\
\hline YNL097C & 0.00 & 4.03 & 0.78 & 0.75 & Nuc \\
\hline YNL098C & 0.94 & 0.64 & 0.00 & 0.64 & Mem \\
\hline YNL129W & 0.00 & 0.54 & 0.00 & 0.54 & Cyt \\
\hline YNL148C & 1.05 & 0.96 & 0.00 & 0.61 & Cyt, Nuc \\
\hline YNL187W & 0.69 & 0.77 & 0.00 & 2.38 & Nuc \\
\hline YNL196C & 1.35 & 1.51 & 0.52 & 0.00 & N/A \\
\hline YNL201C & 0.00 & 0.90 & 0.00 & 0.00 & Nuc \\
\hline YNL211C & 5.22 & 0.58 & 0.00 & 0.64 & Mito \\
\hline YNL212W & 2.24 & 1.28 & 0.59 & 0.00 & Cyt \\
\hline YNL299W & 0.00 & 0.62 & 0.00 & -1.06 & Nuc \\
\hline YNL316C & 2.43 & 0.00 & 0.00 & 0.59 & Cyt \\
\hline YNL322C & 6.07 & 0.00 & 0.00 & 0.71 & Mem \\
\hline YNR050C & 0.60 & 0.00 & 0.00 & 0.60 & Cyt \\
\hline YOL003C & 0.69 & 0.00 & 0.00 & 0.70 & ER \\
\hline YOL059W & 0.00 & 0.69 & 0.58 & 0.00 & Mito \\
\hline YOL070C & 0.00 & 0.66 & 0.00 & 0.69 & Bud neck \\
\hline YOL081W & 6.93 & 4.59 & 0.00 & 0.57 & Mito, Cyt \\
\hline YOL089C & 1.28 & 0.00 & 0.00 & 0.56 & Mito, Nuc \\
\hline YOL106W & 0.66 & 0.00 & 0.00 & 0.56 & N/A \\
\hline YOL118C & 0.00 & 0.51 & 1.05 & 0.00 & N/A \\
\hline YOL128C & 0.68 & 0.00 & 0.00 & 0.53 & N/A \\
\hline YOR051C & 0.00 & 1.23 & 0.00 & 0.57 & Nuc \\
\hline YOR182C & 2.36 & 0.80 & 0.00 & 0.61 & Cyt \\
\hline YOR253W & 0.52 & 0.00 & 0.00 & 0.53 & Cyt \\
\hline YOR265W & 2.68 & 2.12 & 0.00 & -1.06 & Cyt \\
\hline YOR316C & 0.86 & 0.73 & 0.55 & 0.00 & Vac \\
\hline YOR352W & 0.53 & 0.00 & 0.00 & 3.88 & Cyt, Nuc \\
\hline YOR359W & 1.54 & 0.00 & 0.57 & 0.00 & Cyt, Nuc \\
\hline YOR360C & 1.09 & 1.71 & 0.63 & 0.98 & Cyt, Nuc \\
\hline YOR364W & 0.00 & 1.19 & 0.00 & 0.65 & N/A \\
\hline YOR371C & 1.77 & 1.74 & 0.53 & 0.00 & Mem, Cyt \\
\hline YOR384W & 0.76 & 0.00 & 0.00 & 0.52 & Mito \\
\hline YPL002C & 0.00 & 5.19 & 0.00 & 1.08 & Endo \\
\hline YPL037C & 0.68 & 0.00 & 0.00 & 0.51 & Cyt \\
\hline
\end{tabular}




$\begin{array}{llllrl}\text { YPL056C } & 0.00 & 0.67 & 0.55 & 0.00 & \text { N/A } \\ \text { YPL057C } & 0.00 & 1.60 & 0.00 & 2.07 & \text { Mem } \\ \text { YPL065W } & 1.24 & 5.69 & 0.00 & 0.68 & \text { Endo } \\ \text { YPL164C } & 0.57 & 0.00 & 0.00 & 0.72 & \text { Nuc } \\ \text { YPL180W } & 2.38 & 0.00 & 0.00 & 1.47 & \text { Vac } \\ \text { YPL194W } & 0.00 & 0.64 & 0.00 & 0.00 & \text { Nuc } \\ \text { YPL197C } & 0.64 & 0.00 & 0.00 & -1.17 & \text { N/A } \\ \text { YPL215W } & 0.74 & 0.00 & 0.00 & 0.52 & \text { Mito } \\ \text { YPL232W } & 1.08 & 0.00 & 0.00 & 0.71 & \text { Mem } \\ \text { YPL260W } & 0.00 & 0.98 & 0.00 & 0.76 & \text { Cyt, Nuc } \\ \text { YPL269W } & 1.38 & 0.00 & 0.00 & 0.62 & \text { Bud neck } \\ \text { YPR040W } & 2.65 & 0.00 & 0.00 & 0.00 & \text { Cyt, Nuc } \\ \text { YPR173C } & 2.99 & 0.00 & 0.00 & 0.66 & \text { Cyt, Endo } \\ \text { YPR197C } & 0.00 & 10.15 & 0.52 & 0.00 & \text { N/A }\end{array}$




\subsection{Cell death assays: Glucose vs. Glycerol as a carbon source}

The cell death assays were also performed with equisetin. As with TA-289, equisetin killed BY4742 wt (A) and pdrA (B) yeast cells within the first hour of treatment when glucose is used as a carbon source, including wt as equisetin is more potent than TA-289.

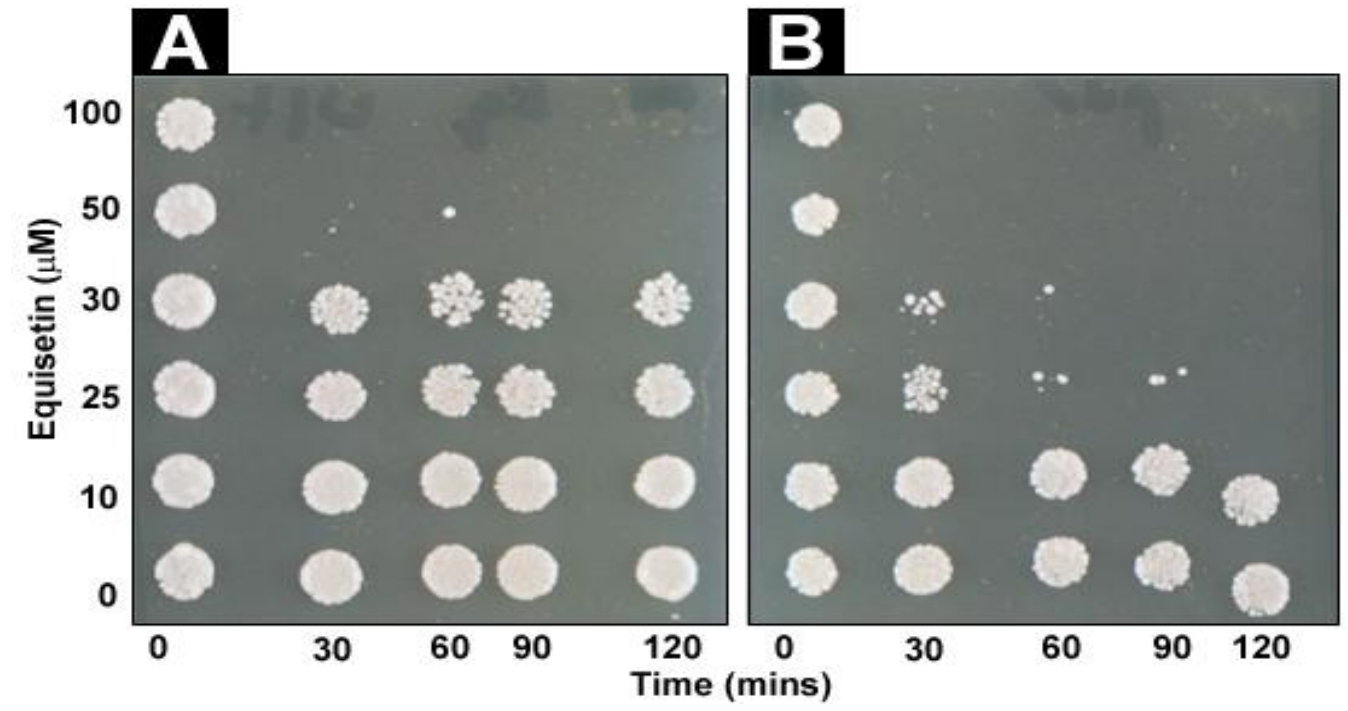

Wherein glycerol-ethanol is used, cells are more resistant to equisetin (below).

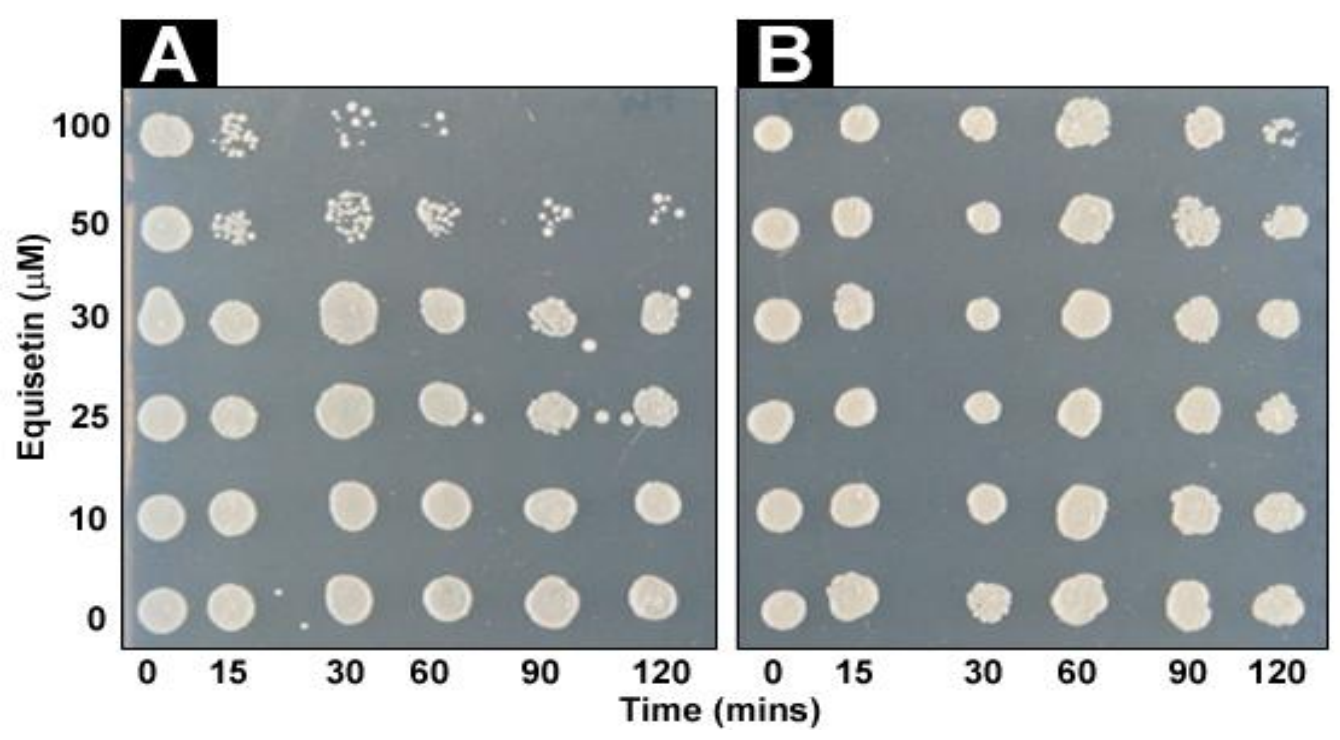




\section{APPENDIX 2}

\subsection{Resistant mutants and cross-resistance scores}

The table below details the 22 TA-289 resistant mutants (TA1-22) and 21 equisetin resistant mutants (EQ1-21) isolated from both EMS mutagenesis and resistance that arose spontaneously. The mutants were tested for cross-resistance to both compounds and were scored according to their level of resistance. TA-289 resistant mutants were sorted according to their resistance score to TA-289 $(60 \mu \mathrm{M})$, and equisetin resistant mutants according to their score to equisetin of the same concentration.

\begin{tabular}{|c|c|c|c|c|c|c|c|c|}
\hline \multirow{3}{*}{\begin{tabular}{|l|} 
Drug \\
TA-289
\end{tabular}} & \multicolumn{4}{|c|}{$\begin{aligned} 1 & =\text { Little growth (Weak resistance) } \\
3 & =\text { Good growth }\end{aligned}$} & \multicolumn{4}{|c|}{$\begin{array}{l}2=\text { Fair growth } \\
4=\text { Near wild-type (Resistant) }\end{array}$} \\
\hline & \multirow{2}{*}{\begin{tabular}{|l|l}
$\begin{array}{l}\text { Mutant } \\
\text { name }\end{array}$ \\
TA3
\end{tabular}} & \multirow{2}{*}{\multicolumn{2}{|c|}{$\begin{array}{l}\begin{array}{l}\text { Spontaneous EMS-treate } \\
\text { mutation }\end{array} \\
\text { mutation }\end{array}$}} & \multicolumn{2}{|c|}{$\begin{array}{l}\text { ed Score on } \\
\text { SC control }\end{array}$} & \multicolumn{2}{|l|}{$\begin{array}{l}\text { Score on } \\
\text { TA-289 }\end{array}$} & $\begin{array}{l}\text { Score on } \\
\text { Equisetin }\end{array}$ \\
\hline & & & & 0 & 4 & 4 & 4 & 3.5 \\
\hline TA-289 & TA1 & & 1 & 0 & 4 & 4 & 4 & 1.5 \\
\hline TA-289 & TA5 & & 1 & 0 & 4 & 4 & 4 & 2 \\
\hline TA-289 & TA8 & & 1 & 0 & 4 & 4 & 4 & 1 \\
\hline TA-289 & TA2 & & 1 & 0 & 4 & 4 & 4 & 0 \\
\hline TA-289 & TA17 & & 0 & 1 & 4 & 4 & 4 & 0 \\
\hline TA-289 & TA20 & & 0 & 1 & 4 & 4 & 4 & 0 \\
\hline TA-289 & TA13 & & 0 & 1 & 4 & 3 & 3.5 & 0.5 \\
\hline TA-289 & TA18 & & 0 & 1 & 4 & 3 & 3.5 & 0 \\
\hline TA-289 & TA10 & & 1 & 0 & 4 & 2 & 2.5 & 3 \\
\hline TA-289 & TA15 & & 0 & 1 & 4 & 2 & 2.5 & 0 \\
\hline TA-289 & TA16 & & 0 & 1 & 4 & 4 & 2 & 0 \\
\hline TA-289 & TA19 & & 0 & 1 & 2 & 2 & 2 & 0 \\
\hline TA-289 & TA21 & & 0 & 1 & 4 & 4 & 2 & 0 \\
\hline TA-289 & TA7 & & 1 & 0 & 4 & 1 & 1.5 & 0 \\
\hline TA-289 & TA4 & & 1 & 0 & 3.5 & & 1.5 & 0 \\
\hline TA-289 & TA6 & & 1 & 0 & 3 & 1 & 1.5 & 0 \\
\hline TA-289 & TA12 & & 1 & 0 & 3.5 & & 1.5 & 0 \\
\hline TA-289 & TA22 & & 0 & 1 & 3.5 & & 1.5 & \\
\hline TA-289 & TA14 & & 0 & 1 & 4 & 4 & 1 & 1.5 \\
\hline TA-289 & TA9 & & 1 & 0 & 4 & 4 & 1 & 1 \\
\hline TA-289 & TA11 & & 1 & 0 & 3 & 3 & 1 & 0 \\
\hline
\end{tabular}




\begin{tabular}{|c|c|c|c|c|c|c|c|c|}
\hline Drug & $\begin{array}{l}\text { Mutant } \\
\text { name }\end{array}$ & $\begin{array}{l}\text { Spontaneous } \\
\text { mutation }\end{array}$ & $\begin{array}{l}\text { EMS-treate } \\
\text { mutation }\end{array}$ & & $\begin{array}{l}\text { core on } \\
\text { C control }\end{array}$ & $\begin{array}{l}\text { Score or } \\
\text { TA-289 }\end{array}$ & & $\begin{array}{l}\text { core on } \\
\text { quisetin }\end{array}$ \\
\hline Equisetin & EQ9 & 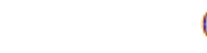 & 0 & 1 & 3 & & 1 & 3 \\
\hline Equisetin & EQ12 & ( & 0 & 1 & 3.5 & & 0 & 3 \\
\hline Equisetin & EQ15 & ( & 0 & 1 & 4 & & 1 & 2 \\
\hline Equisetin & EQ1 & & 1 & 0 & 3.5 & & .5 & 1.5 \\
\hline Equisetin & EQ5 & 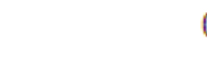 & 0 & 1 & 4 & & 2 & 1.5 \\
\hline Equisetin & EQ7 & ( & 0 & 1 & 4 & & .5 & \\
\hline Equisetin & EQ6 & 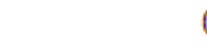 & 0 & 1 & 4 & & .5 & 1 \\
\hline Equisetin & EQ10 & 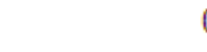 & 0 & 1 & 2 & & 0 & 1 \\
\hline Equisetin & EQ11 & 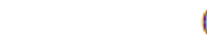 & 0 & 1 & 3 & & .5 & 0.5 \\
\hline Equisetin & EQ2 & & 1 & 0 & 3.5 & & 3 & 0 \\
\hline Equisetin & EQ3 & 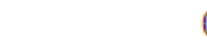 & 0 & 1 & 4 & & 3 & 0 \\
\hline Equisetin & EQ14 & ( & 0 & 1 & 3.5 & & .5 & 0 \\
\hline Equisetin & EQ16 & ( & 0 & 1 & 4 & & .5 & 0 \\
\hline Equisetin & EQ13 & 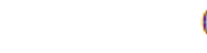 & 0 & 1 & 4 & & 1 & 0 \\
\hline Equisetin & EQ4 & ( & 0 & 1 & 4 & & .5 & 0 \\
\hline Equisetin & EQ8 & ( & 0 & 1 & 4 & & .5 & 0 \\
\hline Equisetin & EQ17 & 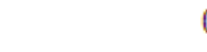 & 0 & 1 & 4 & & .5 & 0 \\
\hline Equisetin & EQ18 & 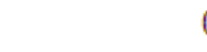 & 0 & 1 & 1 & & .5 & 0 \\
\hline Equisetin & EQ20 & ( & 0 & 1 & 1 & 0 & .5 & 0 \\
\hline Equisetin & $E Q 21$ & 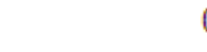 & 0 & 1 & 3 & & .5 & 0 \\
\hline Equisetin & EQ19 & ( & 0 & 1 & 2 & & 0 & 0 \\
\hline
\end{tabular}




\subsection{Generating an isogenic MATa wild-type strain}

\subsubsection{Isogenic wild-type creation and confirmation}

An isogenic MATa strain of the same parental background as the $p d r \Delta$ strain used to generate resistant mutants was created in order to cross with the resistant mutants for dominance/recessiveness studies. This involved transforming a $p d r 14:: k a n R$ yeast strain with a $U R A 3$ deletion cassette containing region homology with the ORFs of PDR3, creating a $p d r 14:: k a n R$ pdr34::URA3 strain (YCG387). The URA3 deletion cassette was amplified from a purified pAG60 carrier plasmid by PCR. The figure below shows the expected band sizes for the pAG60 plasmid (4000 bp) and the amplified $U R A 3$ deletion cassette (1600 bp).

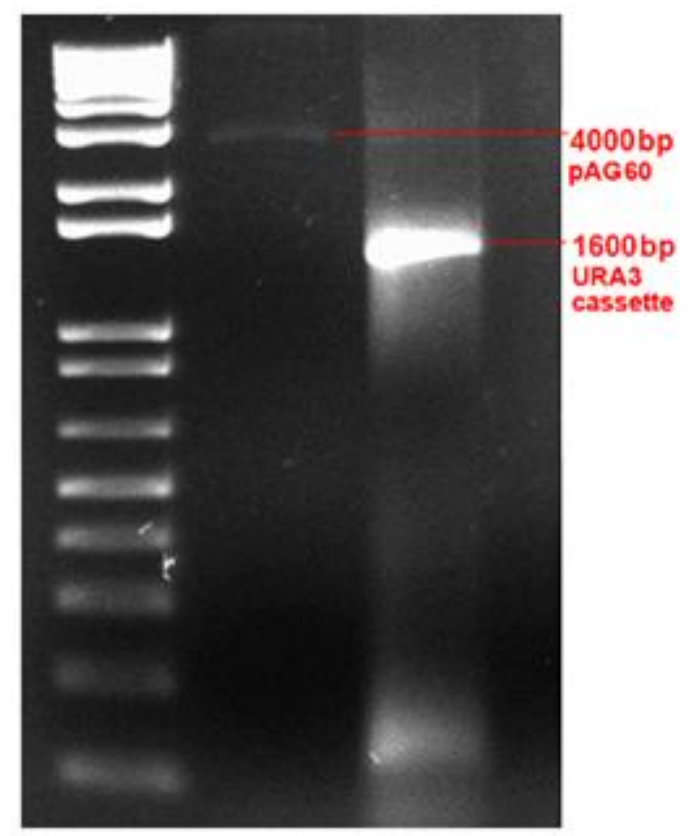


Then, the pdr1 $1 \triangle:$ kan $R$ strain was transformed with the PCR product, after which a colony PCR was performed to confirm the correct insertion of the $U R A 3$ cassette (and thus the deletion of $P D R 3$ ). Expected band sizes (880 bp and $710 \mathrm{bp}$ ) of both forward and reverse internal and external primers observed under gel electrophoresis validates that the transformation was indeed successful.

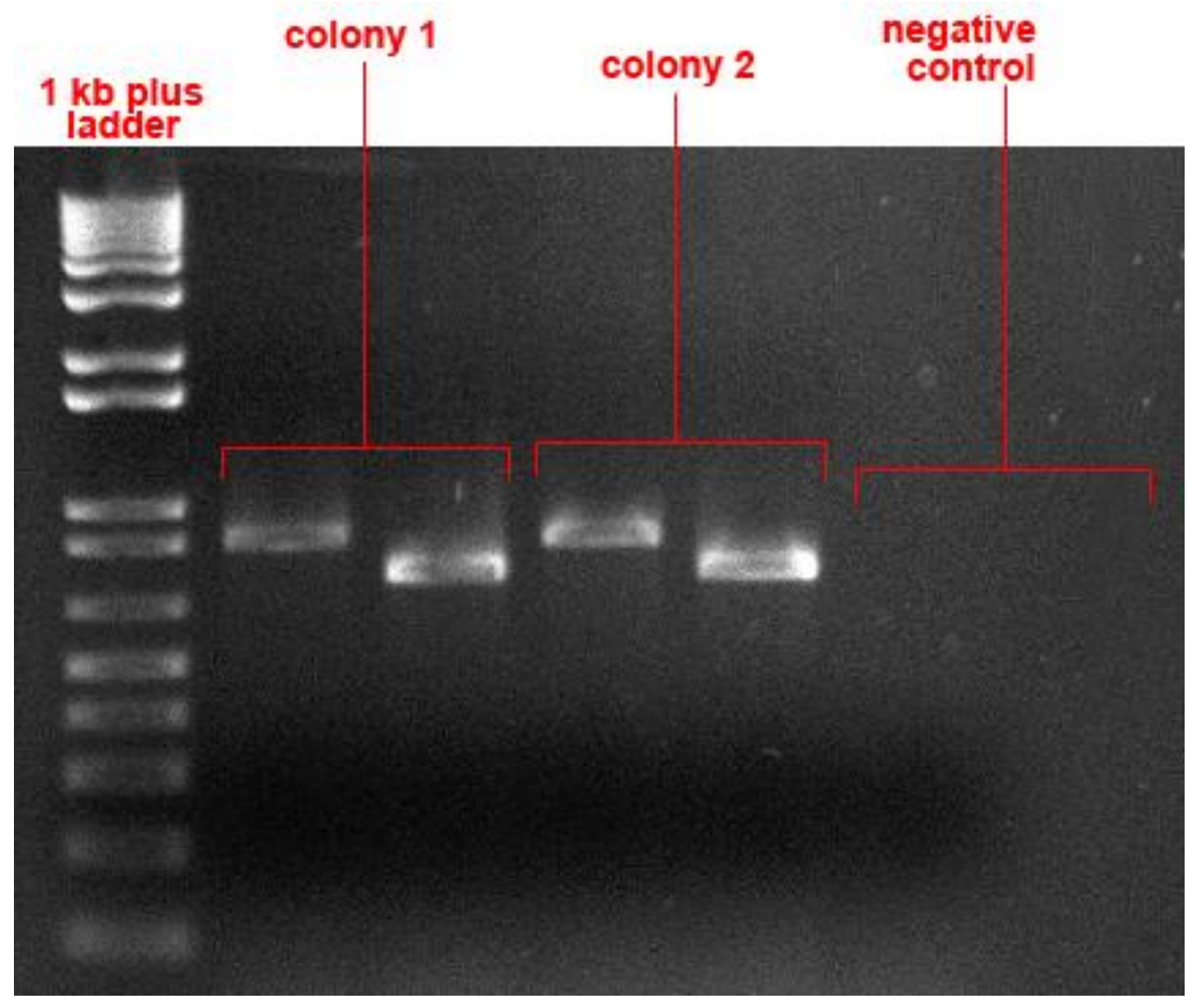

\subsubsection{Making heterozygous diploid mutants}

Heterozygous diploid mutants were generated by crossing the isogenic wt strain YCG387 with the selected resistant mutant, TA8. This was achieved by chevron creation on rich media, then replica-plated onto selection media. To select for 
diploids specifically, diploid strains (successful mating of YCG387 with the resistant mutants) were selected on SD -Ura + G418 +NAT. These heterozygous diploids were then re-streaked onto SD -Ura $+\mathrm{G} 418+\mathrm{NAT}$ petri plates to attain single colonies for determining dominance and/or recessiveness of the mutants. See figure below for the method of chevron creation and selection of heterozygous diploids.

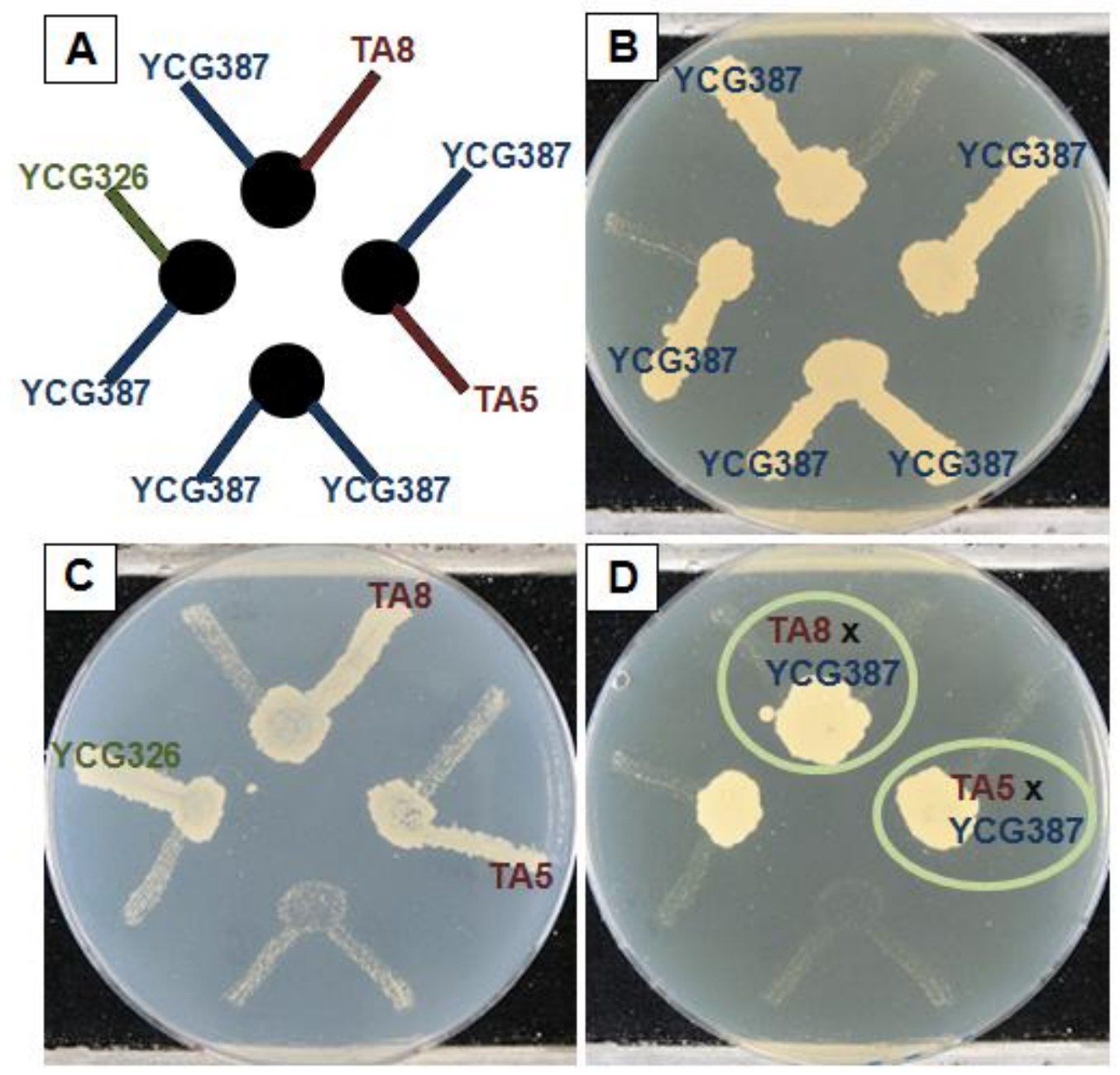

(A) Method of chevron creation; (B) YPD +G418 selection to select for YCG387; (C) SD -Ura +NAT to select for parental strain YCG326 and the resistant mutants; (D) Diploid selection on SD -Ura +G418 +NAT. 


\subsection{Calculating growth rates}

The growth rate of the TA8 mutant was determined and compared to parental pdr $\Delta$ cells. OD of both strains was plotted against time, and the area of exponential growth for both strains was used to calculate growth rates.

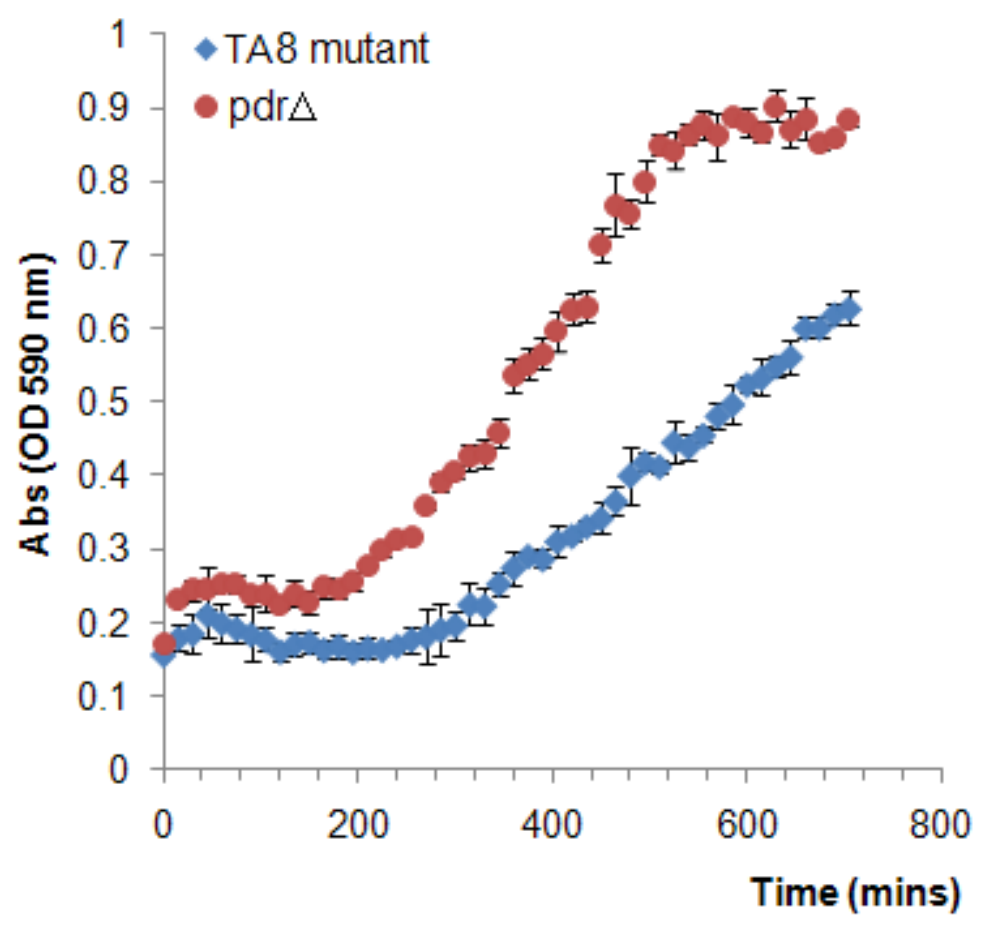

The model equation for exponential growth used was:

$N t=N i \cdot e^{\mu t}$

Where $N t$ is the OD value at any given time point, $N i$ is the y-intercept, $\mu$ is the gradient, and $t$ is time. This was log-transformed to fit a straight line:

$\ln N t=\mu t+\ln N i$

The gradient and $\mathrm{y}$-intercept for both TA8 and pdr $\Delta$ strains were obtained by performing a LINEST function in Microsoft Excel on the log-transformed data: 


\begin{tabular}{|l|l|l|}
\hline Strain & y-intercept $(\ln \boldsymbol{N} \boldsymbol{i})$ & gradient $(\mu)$ \\
\hline TA8 & -2.4 & 0.003 \\
\hline$p d r \Delta$ & -2.08 & 0.0039 \\
\hline
\end{tabular}

Using these values, the doubling time for the TA8 mutant strain was calculated as follows:

Therefore $\ln N t=0.003 t+(-2.4)$

When $\ln N t=-2.4, \mathrm{t}=0$. When $\ln N t=-1.2($ double of -2.4$), \mathrm{t}=\chi$.

Therefore $t=(-1.2)+2.4 / 0.003$

Therefore $t=400$ mins $/ 60$ mins

Therefore $\mathrm{t}=6.6 \mathrm{~h}$

The doubling time for the $p d r \Delta$ parental strain was calculated as follows:

$\ln N t=0.0039 t+(-2.08)$

When $\ln N t=-2.08, \mathrm{t}=0$. When $\ln N t=-1.04($ double of -2.08$), \mathrm{t}=\chi$.

Therefore $t=(-1.04)+2.08 / 0.0039$

Therefore $t=260$ mins $/ 60$ mins

Therefore $\underline{\mathrm{t}=\mathbf{4 . 3 \mathrm { h }}}$ 


\section{APPENDIX 3}

\subsection{High salt does not sequester potency of TA-289 and equisetin}

The figures below indicate $24 \mathrm{~h}$ liquid dose response assays performed with TA-289 and equisetin in the presence (red squares) or absence (green triangles) of high salt (0.7 $\mathrm{M} \mathrm{NaCl}$ ). Both wt (A above and below) and pdrA (B above and below) strains were tested.
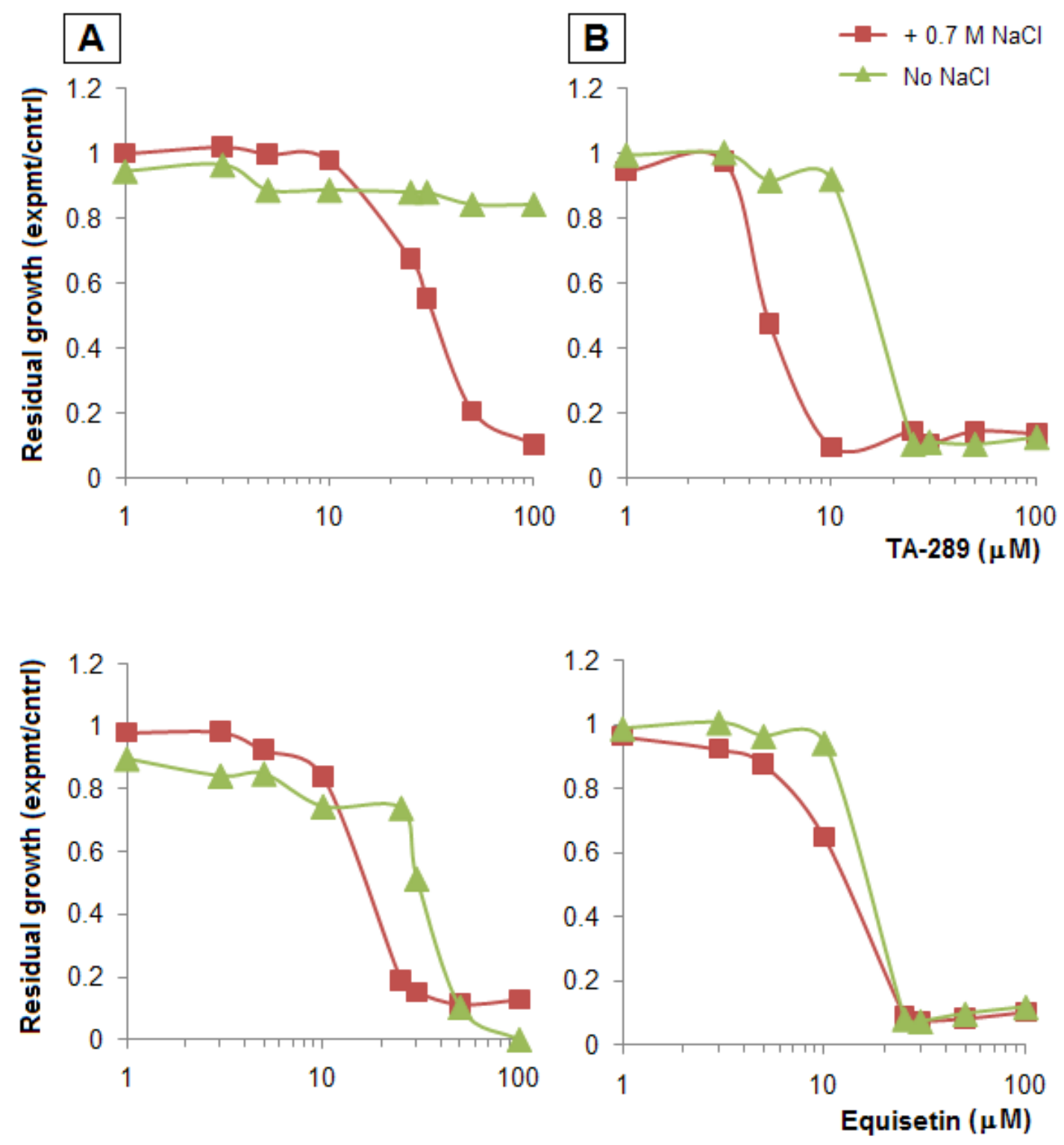


\section{LIST OF REFERENCES}

Ahmed, K. A. and R. A. Woods (1967). "A genetic analysis of resistance to nystatin in Saccharomyces cerevisiae." Genetical Research 9(2): 179-\&.

Akita, M., Shinkai, A., Matsuyama, S.I., Mizushima, S. (1991). "SecA, an essential component of the secondary machinery of Escherichia coli, exists as a homodimer." Biochem Biophys. Res. Comm 174: 211-216.

Alamgir, M., V. Erukova, et al. (2010). "Chemical-genetic profile analysis of five inhibitory compounds in yeast." BMC Chemical Biology 10(1): 6.

Alfatafta, A. A., J. B. Gloer, et al. (1994). "Apiosporamide, a new antifungal agent from the coprophilous fungus Apiospora montagnei." Journal of Natural Products-Lloydia 57(12): 1696-1702.

Alic, N., T. Felder, et al. (2004). "Genome-wide transcriptional responses to a lipid hydroperoxide: adaptation occurs without induction of oxidant defenses." Free Radical Biology and Medicine 37(1): 23-35.

Alpert, D. and J. Vilcek (2000). "Inhibition of IkappaB kinase activity by sodium salicylate in vitro does not reflect its inhibitory mechanism in intact cells." Journal of Biological Chemistry 275(15): 10925-10929.

Altmann, K. and B. Westermann (2005). "Role of essential genes in mitochondrial morphogenesis in Saccharomyces cerevisiae." Mol. Biol. Cell 16(11): 54105417.

Amberg, D., D. Burke, et al. (2005). Methods in yeast genetics : A Cold Spring Harbor Laboratory course manual. Cold Spring Harbor, N.Y., Cold Spring Harbor Laboratory Press.

Amberg, D. C., Burke, D.J., Strathern J.N. (2005). "Methods in yeast genetics : a Cold Spring Harbor Laboratory course manual." Cold Spring Harbor Laboratory Press. 
Andreyev, A. Y., Kushnareva, Y.E., Starkov, A.A. (2005). "Mitochondrial metabolism of reactive oxygen species." Biochemistry (Moscow) 70(2): 200214.

Anke, H. (1979). "Metabolic products of microorganisms. 184. On the mode of action of cladosporin." Journal of Antibiotics 32(9): 952-958.

Ardail, D., F. Gasnier, et al. (1993). "Involvement of mitochondrial contact sites in the subcellular compartmentalization of phospholipid biosynthetic enzymes." Journal of Biological Chemistry 268(34): 25985-25992.

Auerbach, D., A. Arnoldo, et al. (2005). "Drug discovery using yeast as a model system: A functional genomic and proteomic view." Current Proteomics 2: 113.

Ayscough, K. R., J. Stryker, et al. (1997). "High rates of actin filament turnover in budding yeast and roles for actin in establishment and maintenance of cell polarity revealed using the actin inhibitor latrunculin-A." Journal of Cell Biology 137(2): 399-416.

Baetz, K., L. McHardy, et al. (2004). "Yeast genome-wide drug-induced haploinsufficiency screen to determine drug mode of action." Proceedings of the National Academy of Sciences of the United States of America 101(13): 4525-4530.

Baker, G. B., T. I. Prior, et al. (2002). "Chirality and drugs used to treat psychiatric disorders." Journal of Psychiatry \& Neuroscience 27(6): 401-403.

Balzi, E., W. Chen, et al. (1987). "The multidrug resistance gene PDR1 from Saccharomyces cerevisiae." L Biol Chem 262: 16871 - 16879.

Balzi, E. and A. Goffeau (1995). "Yeast multidrug-resistance - the PDR network." Journal of Bioenergetics and Biomembranes 27(1): 71-76.

Barros, M. H., B. Bandy, et al. (2004). "Higher respiratory activity decreases mitochondrial reactive oxygen release and increases life span in Saccharomyces cerevisiae." Journal of Biological Chemistry 279(48): 4988349888. 
Barros, M. H., L. E. S. Netto, et al. (2003). " $\mathrm{H}_{2} \mathrm{O}_{2}$ generation in Saccharomyces cerevisiae respiratory pet mutants: Effect of cytochrome c." Free Radical Biology and Medicine 35(2): 179-188.

Batandier, C., E. Fontaine, et al. (2002). "Determination of mitochondrial reactive oxygen species: methodological aspects." Journal of Cellular and Molecular Medicine 6(2): 175-187.

Batova, M., V. Klobucnikova, et al. (2010). "Chemogenomic and transcriptome analysis identifies mode of action of the chemosensitizing agent CTBT (7chlorotetrazolo[5,1-c]benzo[1,2,4]triazine)." Bmc Genomics 11(1): 153.

Belikova, N. A., Y. A. Vladimirov, et al. (2006). "Peroxidase activity and structural transitions of cytochrome c bound to cardiolipin-containing membranes." Biochemistry 45(15): 4998-5009.

Bertina, R. M., J. A. Steenstra, et al. (1974). "The mechanism of inhibition by oligomycin of oxidative phosphorylation in mitochondria." Biochimica et Biophysica Acta (BBA) - Bioenergetics 368(3): 279-297.

Bhikshapathi, D., Y. S. Kumar, et al. (2010). "Borrelidin: A prospective drug." Indian Journal of Biotechnology 9(1): 18-23.

Bieker, K. L., Phillips, G.J., Silhavy, T.J. (1990). "The sec and prl genes of Escherichia coli." Journal of Bioenergetics and Biomembranes 22(3): 291-310.

Binder, M. and C. Tamm (1973). "Cytochalasans - new class of biologically-active microbial metabolites." Angewandte Chemie-International Edition in English 12(5): 370-380.

Birrell, G. W., J. A. Brown, et al. (2002). "Transcriptional response of Saccharomyces cerevisiae to DNA-damaging agents does not identify the genes that protect against these agents." Proceedings of the National Academy of Sciences of the United States of America 99(13): 8778-8783.

Bloor, S. (2008). "Arthrinic acid, a novel antifungal polyhydroxyacid from Arthrinium phaeospermum." Journal of Antibiotics 61(8): 515-517. 
Blunt, J. W., B. R. Copp, et al. (2008). "Marine natural products." Natural Product Reports 25(1): 35-94.

Boisrame, A., M. Chasles, et al. (2002). "Sbh1p, a subunit of the Sec61 translocon, interacts with the chaperone calnexin in the yeast Yarrowia lipolytica." Journal of Cell Science 115(24): 4947-4956.

Boone, C., H. Bussey, et al. (2007). "Exploring genetic interactions and networks with yeast." Nature Reviews Genetics 8(6): 437-449.

Botchkarev, V. (2003). "Molecular mechanisms of chemotherapy-induced hair loss." Journal of Investigative Dermatology Symposium Proceedings 8(1): 72-75.

Bradner, J. E., O. M. McPherson, et al. (2006). "A method for the covalent capture and screening of diverse small molecules in a microarray format." Nat. Protocols 1(5): 2344-2352.

Burke, L. T., D. J. Dixon, et al. (2005). "Total synthesis of the Fusarium toxin equisetin." Organic \& Biomolecular Chemistry 3(2): 274-280.

Burmeister, H. R., G. A. Bennett, et al. (1974). "Antibiotic produced by Fusarium equiseti NRRL 5537." Antimicrob. Agents Chemother. 5(6): 634-639.

Butcher, R. A., B. S. Bhullar, et al. (2006). "Microarray-based method for monitoring yeast overexpression strains reveals small-molecule targets in TOR pathway." Nature Chemical Biology 2(2): 103-109.

Butcher, R. A. and S. L. Schreiber (2003). "A small molecule suppressor of FK506 that targets the mitochondria and modulates ionic balance in Saccharomyces cerevisiae." Chemistry \& Biology 10(6): 521-531.

Butler, M. S. (2004). "The role of natural product chemistry in drug discovery." Journal of Natural Products 67(12): 2141-2153.

Cabelli, R. J., Dolan, K.M., Qian, L., Oliver, D.B. (1991). "Characterization of membrane-associated and soluble states of SecA protein from wild-type and SecA52 (Ts) mutant strains of Escherichia coli." Journal of Biological Chemistry 266: 24420-24427. 
Cardenas, M. E., M. C. Cruz, et al. (1999). "Antifungal activities of antineoplastic agents: Saccharomyces cerevisiae as a model system to study drug action." Clin. Microbiol. Rev. 12(4): 583-611.

Cardone, J. M., L. F. Revers, et al. (2006). "Psoralen-sensitive mutant pso9-1 of Saccharomyces cerevisiae contains a mutant allele of the DNA damage checkpoint gene MEC3." DNA Repair 5(2): 163-171.

Cary, J. W., K. C. Ehrlich, et al. (2009). "Characterization of the Aspergillus ochraceoroseus aflatoxin/sterigmatocystin biosynthetic gene cluster." Mycologia 101(3): 352-362.

Chan, T. F., J. Carvalho, et al. (2000). "A chemical genomics approach toward understanding the global functions of the target of rapamycin protein (TOR)." Proceedings of the National Academy of Sciences of the United States of America 97(24): 13227-13232.

Chan, W. H. (2007). "Citrinin induces apoptosis via a mitochondria-dependent pathway and inhibition of survival signals in embryonic stem cells, and causes developmental injury in blastocysts." Biochemical Journal 404: 317326.

Chen, M. J., K. Narkunan, et al. (1999). "Total synthesis of natural bicyclic lactones (+)-dihydrocanadensolide, (+/-)-avenociolide, and (+/-)-isoavenociolide via tungsten-pi-allyl complexes." Journal of Organic Chemistry 64(22): 83118318.

Chen, Q., E. J. Vazquez, et al. (2003). "Production of reactive oxygen species by mitochondria." Journal of Biological Chemistry 278(38): 36027-36031.

Chen, S.-R., D. D. Dunigan, et al. (2003). "Bcl-2 family members inhibit oxidative stress-induced programmed cell death in Saccharomyces cerevisiae." Free Radical Biology and Medicine 34(10): 1315-1325.

Chiu, J., C. M. Tactacan, et al. (2011). "Cell cycle sensing of oxidative stress in Saccharomyces cerevisiae by oxidation of a specific cysteine residue in the 
transcription factor Swi6p." Journal of Biological Chemistry 286(7): 52045214.

Chou, W. G. and B. M. Pogell (1981). "Mode of action of pamamycin in Stapphylococcus aureus." Antimicrobial Agents and Chemotherapy 20(4): 443-454.

Chou, W. G. and B. M. Pogell (1981). "Pamamycin inhibits nucleoside and inorganic-phosphate transport in Stapphylococcus aureus." Biochemical and Biophysical Research Communications 100(1): 344-350.

Clardy, J. and C. Walsh (2004). "Lessons from natural molecules." Nature 432(7019): 829-837.

Cocheme, H. and M. Murphy (2008). "Complex I is the major site of mitochondrial superoxide production by paraquat." J Biol Chem 283: 1786 - 1798.

Collins, S., M. Schuldiner, et al. (2006). "A strategy for extracting and analyzing large-scale quantitative epistatic interaction data." Genome Biology 7(7): 114.

Cui, Z., D. Hirata, et al. (1996). "The multidrug resistance-associated protein (MRP) subfamily (Yrs1/Yor1) of Saccharomyces cerevisiae is important for the tolerance to a broad range of organic anions." Journal of Biological Chemistry 271(25): 14712-14716.

Davey, H. M. and P. Hexley (2010). "Red but not dead? Membranes of stressed Saccharomyces cerevisiae are permeable to propidium iodide." Environmental Microbiology 13(1): 163-171.

Dawes, I. W. (2004). Yeast stress responses. Boca Raton, CRC Press.

Decuypere, J. P., Monaco, G., Missiaen, L., De Smedt, H., Parys, J.B., Bultynck, G. (2011). "IP3 Receptors, Mitochondria, and Ca2+ Signaling: Implications for Aging." Journal of Aging Research: 1-20.

Delhumeau, G., A. M. Cruzmendoza, et al. (1994). "Protection of cytochrome $c$ oxidase against cyanide inhibition by pyruvate and [alpha]-ketoglutarate: 
Effect of aeration in vitro." Toxicology and Applied Pharmacology 126(2): 345-351.

Denning, D. W. (2003). "Echinocandin antifungal drugs." Lancet 362(9390): 11421151.

Desai, K., M. C. Sullards, et al. (2002). "Fumonisins and fumonisin analogs as inhibitors of ceramide synthase and inducers of apoptosis." Biochimica Et Biophysica Acta-Molecular and Cell Biology of Lipids 1585(2-3): 188-192.

Dimmer, K. S., S. Fritz, et al. (2002). "Genetic basis of mitochondrial function and morphology in Saccharomyces cerevisiae." Mol. Biol. Cell 13(3): 847-853.

Dorer, R. K., S. Zhong, et al. (2005). "A small-molecule inhibitor of Mps1 blocks the spindle-checkpoint response to a lack of tension on mitotic chromosomes." Current Biology 15(11): 1070-1076.

Drakulic, T., M. D. Temple, et al. (2005). "Involvement of oxidative stress response genes in redox homeostasis, the level of reactive oxygen species, and ageing in Saccharomyces cerevisiae." FEMS Yeast Research 5(12): 1215-1228.

Drews, J. (2000). "Drug discovery: A historical perspective." Science 287(5460): 1960-1964.

Duggar, B. M. (1948). "Aureomycin: A product of the continuing search for new antibiotics." Annals of the New York Academy of Sciences 51(2): 177-181.

Eble, K. S., W. B. Coleman, et al. (1990). "Tightly associated cardiolipin in the bovine heart mitochondrial ATP synthase as analyzed by 31P nuclear magnetic resonance spectroscopy." Journal of Biological Chemistry 265(32): 19434-19440.

Eisenberg, T., S. Büttner, et al. (2007). "The mitochondrial pathway in yeast apoptosis." Apoptosis 12(5): 1011-1023.

Elrod, J. W., R. Wong, et al. (2010). "Cyclophilin D controls mitochondrial poredependent $\mathrm{Ca} 2+$ exchange, metabolic flexibility, and propensity for heart failure in mice." The Journal of Clinical Investigation 120(10): 3680-3687. 
Feher, M. and J. M. Schmidt (2003). "Property distributions: Differences between drugs, natural products, and molecules from combinatorial chemistry." Journal of Chemical Information and Computer Sciences 43(1): 218-227.

Flamee, P. A. (1985). "The action of actinomycin D on the transcription of T7 coliphage DNA by Escherichia coli RNA polymerase." Biochemical Journal 230(2): 557-560.

Flattery-O'Brien, J. A. and I. W. Dawes (1998). "Hydrogen peroxide causes RAD9dependent cell cycle arrest in $\mathrm{G}_{2}$ in Saccharomyces cerevisiae whereas menadione causes $\mathrm{G}_{1}$ arrest independent of RAD9 function." Journal of Biological Chemistry 273(15): 8564-8571.

Foury, F. (1997). "Human genetic diseases: A cross-talk between man and yeast." Gene 195(1): 1-10.

Fried, H. M. and J. R. Warner (1981). "Cloning of yeast gene for trichodermin resistance and ribosomal protein L3." Proceedings of the National Academy of Sciences of the United States of America 78(1): 238-242.

Fried, H. M. and J. R. Warner (1982). "Molecular-cloning and analysis of yeast gene for cycloheximide resistance and ribosomal-protein L29." Nucleic Acids Research 10(10): 3133-3148.

Funder, J. W. (2005). "The nongenomic actions of aldosterone." Endocr. Rev. 26(3): 313-321.

Gaigg, B., R. Simbeni, et al. (1995). "Characterisation of a microsomal subfraction associated with mitochondria of the yeast, Saccharomyces cerevisiae: Involvement in synthesis and import of phospholipids into mitochondria." Biochimica Et Biophysica Acta-Biomembranes 1234(2): 214-220.

Ghannoum, M. and L. Rice (1999). "Antifungal agents: mode of action, mechanisms of resistance, and correlation of these mechanisms with bacterial resistance." Clin Microbiol Rev 12: 501 - 517.

Giaever, G., A. M. Chu, et al. (2002). "Functional profiling of the Saccharomyces cerevisiae genome." Nature 418(6896): 387-391. 
Giaever, G., D. D. Shoemaker, et al. (1999). "Genomic profiling of drug sensitivities via induced haploinsufficiency." Nature Genetics 21(3): 278-283.

Gietz, R. D. and R. H. Schiestl (2007). "High-efficiency yeast transformation using the LiAc/SS carrier DNA/PEG method." Nat. Protocols 2(1): 31-34.

Gottesman, M. M. (2002). "Mechanisms of cancer drug resistance." Annual Review of Medicine 53: 615-627.

Gralla, E. B., D. J. Kosman, et al. (1992). Molecular genetics of superoxide dismutases in yeasts and related fungi. Advances in Genetics, Academic Press. Volume 30: 251-319.

Grozinger, C. M., Chao, E.D., Blackwell, H.E., Mozed, D., Schreiber, S.L. (2001). "Identification of a class of small molecule inhibitors of the sirtuin family of NAD-dependent deacetylases by phenotypic screening." Journal of Biological Chemistry 276(42): 38837-38843.

Gullo, V. P., J. McAlpine, et al. (2006). "Drug discovery from natural products." Lournal of Industrial Microbiology \& Biotechnology 33(7): 523-531.

Haase, S. B. (2001). Cell cycle analysis of budding yeast using SYTOX green, John Wiley \& Sons, Inc.

Haber, F. and J. Weiss (1932). "Über die Katalyse des Hydroperoxydes." Naturwissenschaften 20(51): 948-950.

Haines, T. H. and N. A. Dencher (2002). "Cardiolipin: a proton trap for oxidative phosphorylation." FEBS Letters 528(1-3): 35-39.

Halliwell, B. (1995). The biological significant of oxygen-derived species. Glasgow, Blackie Academic and Professional.

Hamel, E. (1996). "Antimitotic natural products and their interactions with tubulin." Medicinal Research Reviews 16(2): 207-231.

Haynes, C. M., E. A. Titus, et al. (2004). "Degradation of misfolded proteins prevents ER-derived oxidative stress and cell death." Molecular cell 15(5): 767-776. 
Hazuda, D., C. U. Blau, et al. (1999). "Isolation and characterization of novel human immunodeficiency virus integrase inhibitors from fungal metabolites." Antiviral Chemistry \& Chemotherapy 10(2): 63-70.

Heidler, S. and J. Radding (1995). "The AUR1 gene in Saccharomyces cerevisiae encodes dominant resistance to the antifungal agent aureobasidin A (LY295337)." Antimicrob. Agents Chemother. 39(12): 2765-2769.

Heitman, J., N. R. Movva, et al. (1991). "Targets for cell-cycle arrest by the immunosuppressant rapamycin in yeast." Science 253(5022): 905-909.

Hellwig, V., T. Grothe, et al. (2003). "Altersetin, a new antibiotic from cultures of endophytic Alternaria spp. Taxonomy, fermentation, isolation, structure elucidation and biological activities." ChemInform 34(12): no-no.

Hillenmeyer, M. E., E. Fung, et al. (2008). "The chemical genomic portrait of yeast: Uncovering a phenotype for all genes." Science 320(5874): 362-365.

Ho, C. H., L. Magtanong, et al. (2009). "A molecular barcoded yeast ORF library enables mode-of-action analysis of bioactive compounds." Nature Biotechnology 27(4): 369-377.

Hoffmann, H.-P. and C. J. Avers (1973). "Mitochondrion of yeast: Ultrastructural evidence for one giant, branched organelle per cell." Science 181(4101): 749751.

Hood, K. A., L. M. West, et al. (2002). "Peloruside A, a novel antimitotic agent with paclitaxel-like microtubule-stabilizing activity." Cancer Research 62(12): 3356-3360.

Houtkooper, R. and F. Vaz (2008). "Cardiolipin, the heart of mitochondrial metabolism." Cellular and Molecular Life Sciences 65(16): 2493-2506.

Hudson, J. R., E. P. Dawson, et al. (1997). "The complete set of predicted genes from Saccharomyces cerevisiae in a readily usable form." Genome Research 7(12): 1169-1173.

Hughes, T. (2002). "Yeast and drug discovery." Functional \&amp; Integrative Genomics 2(4): 199-211. 
Huh, W.-K., J. V. Falvo, et al. (2003). "Global analysis of protein localization in budding yeast." Nature 425(6959): 686-691.

Iwahashi, H., E. Kitagawa, et al. (2007). "Evaluation of toxicity of the mycotoxin citrinin using yeast ORF DNA microarray and Oligo DNA microarray." $\underline{\mathrm{Bmc}}$ Genomics 8.

Iwasaki, S. (1992). "Antimitotic agents: Chemistry and recognition of tubulin molecule." ChemInform 23(17): no-no.

Jayakody, L., N. Hayashi, et al. (2010). "Identification of glycolaldehyde as the key inhibitor of bioethanol fermentation by yeast and genome-wide analysis of its toxicity." Biotechnology Letters 33(2): 285-292.

Jiang, F., M. T. Ryan, et al. (2000). "Absence of cardiolipin in the crd1 null mutant results in decreased mitochondrial membrane potential and reduced mitochondrial function." Journal of Biological Chemistry 275(29): 2238722394.

Jones, K. H. and J. A. Senft (1985). "An improved method to determine cell viability by simultaneous staining with fluorescein diacetate-propidium iodide." Journal of Histochemistry \& Cytochemistry 33(1): 77-9.

Jorgensen, P., B. Nelson, et al. (2002). "High-resolution genetic mapping with ordered arrays of Saccharomyces cerevisiae deletion mutants." Genetics 162(3): 1091-1099.

Jouvet, N., J. Poschmann, et al. (2010). "Rrd1 isomerizes RNA polymerase II in response to rapamycin." BMC Molecular Biology 11(1): 92.

Kaaufer, N. F., H. M. Fried, et al. (1983). "Cycloheximide resistance in yeast: the gene and its protein." Nucleic Acids Research. 11(10): 3123-3135.

Koehn, F. E. and G. T. Carter (2005). "The evolving role of natural products in drug discovery." Nature Reviews Drug Discovery 4(3): 206-220.

Kojro, E., P. Fuger, et al. (2010). "Statins and the squalene synthase inhibitor zaragozic acid stimulate the non-amyloidogenic pathway of amyloid-beta 
protein precursor processing by suppression of cholesterol synthesis." Journal of Alzheimers Disease 20(4): 1215-1231.

König, T., A. Kapus, et al. (1993). "Effects of equisetin on rat liver mitochondria: Evidence for inhibition of substrate anion carriers of the inner membrane." Journal of Bioenergetics and Biomembranes 25(5): 537-545.

Kopp, E. and S. Ghosh (1994). "Inhibition of NF-kappa B by sodium salicylate and aspirin." Science 265(5174): 956-959.

Koppenol, W. H. (2001). "The Haber-Weiss cycle: 70 years later." Redox Rep 16(4): 229-34.

Korshunov, S. S., V. P. Skulachev, et al. (1997). "High protonic potential actuates a mechanism of production of reactive oxygen species in mitochondria." FEBS Letters 416(1): 15-18.

Kumar, N., M. Kiuchi, et al. (2005). "Small-molecule diversity using a skeletal transformation strategy." Organic Letters 7(13): 2535-2538.

Kushnareva, Y., A. N. Murphy, et al. (2002). "Complex I-mediated reactive oxygen species generation: Modulation by cytochrome $c$ and $\mathrm{NAD}(\mathrm{P})^{+}$oxidationreduction state." Biochemical Journal 384: 545-553.

Kwak, Y.-S., S. Han, et al. (2011). "Saccharomyces cerevisiae genome-wide mutant screen for sensitivity to 2,4-diacetylphloroglucinol, an antibiotic produced by Pseudomonas fluorescens." Appl. Environ. Microbiol. 77(5): 1770-1776.

Lai, B., L. Zhang, et al. (2005). "Inhibition of Qi site of mitochondrial complex III with antimycin A decreases persistent and transient sodium currents via reactive oxygen species and protein kinase C in rat hippocampal CA1 cells." Experimental Neurology 194(2): 484-494.

Langston, J. W., Forno, L.S., Tetrud, J., Reeves, A.G., Kaplan, J.A., Karluk, D. (1999). "Evidence of active nerve cell degeneration in the substantia nigra of humans years after 1-methyl-4-phenyl-1,2,3,6-tetrahydropyridine exposure." Annals of Neurology 46(4): 598-605. 
Laport, M. S., O. C. S. Santos, et al. (2009). "Marine sponges: Potential sources of new antimicrobial drugs." Current Pharmaceutical Biotechnology 10(1): 86105.

Larsen, T. O., J. Smedsgaard, et al. (2005). "Phenotypic taxonomy and metabolite profiling in microbial drug discovery." Natural Product Reports 22(6): 672695.

Launhardt, H., A. Hinnen, et al. (1998). "Drug-induced phenotypes provide a tool for the functional analysis of yeast genes." Yeast 14(10): 935-942.

Li, N., K. Ragheb, et al. (2003). "Mitochondrial complex I inhibitor rotenone induces apoptosis through enhancing mitochondrial reactive oxygen species production." Journal of Biological Chemistry 278(10): 8516-8525.

Li, W., W. Mo, et al. (2005). "Yeast model uncovers dual roles of mitochondria in the action of artemisinin." PLoS Genet 13.

Luesch, H., T. Y. H. Wu, et al. (2005). "A genome-wide overexpression screen in yeast for small-molecule target identification." Chemistry \& Biology 12(1): 55-63.

Lum, P. Y., C. D. Armour, et al. (2004). "Discovering modes of action for therapeutic compounds using a genome-wide screen of yeast heterozygotes." Cell 116(1): 121-137.

Lundin, M., S. W. Deopujari, et al. (1992). "Characterization of a mitochondrial inorganic pyrophosphatase in Saccharomyces cerevisiae." Biochimica Et Biophysica Acta 1098(2): 217-223.

Lundin, M., S. W. Deopujari, et al. (1992). "Characterization of a mitochondrial inorganic pyrophosphatase in Saccharomyces cerevisiae." Biochimica et Biophysica Acta (BBA) - Bioenergetics 1098(2): 217-223.

Machida, K. and T. Tanaka (1999). "Farnesol-induced generation of reactive oxygen species dependent on mitochondrial transmembrane potential hyperpolarization mediated by F0F1-ATPase in yeast." FEBS Letters 462(1-2): 108-112. 
Machida, K., T. Tanaka, et al. (1998). "Farnesol-induced generation of reactive oxygen species via indirect inhibition of the mitochondrial electron transport chain in the yeast Saccharomyces cerevisiae." Journal of Bacteriology 180(17): 4460-4465.

Madeo, F., D. Carmona-Gutierrez, et al. (2009). "Caspase-dependent and caspaseindependent cell death pathways in yeast." Biochemical and Biophysical Research Communications 382(2): 227-231.

Madeo, F., E. Frohlich, et al. (1999). "Oxygen stress: A regulator of apoptosis in yeast." Journal of Cell Biology 168: 353-358.

Madeo, F., E. Herker, et al. (2002). "A caspase-related protease regulates apoptosis in yeast." Molecular cell 9(4): 911-917.

Madeo, F., E. Herker, et al. (2004). "Apoptosis in yeast." Current Opinion in Microbiology 7(6): 655-660.

Maia, J. (1994). "Hexosamine and cell wall biogenesis in the aquatic fungus Blastocladiella emersonii." FASEB I 8(11): 848-853.

Marfori, E. C., S. i. Kajiyama, et al. (2003). "Phytotoxicity of the tetramic acid metabolite trichosetin." Phytochemistry 62(5): 715-721.

Marfori, E. C., Kajiyama, S., Fukusaki, E., Kobayashi A. (2002). "Trichosetin,a novel tetramic acid antibiotic produced in dual culture of Trichoderma harzianum and Catharanthus roseus callus." Verlag der Zeitschrift für Naturforschung 57: $465-470$.

Middlekauff, J. E., S. Hino, et al. (1957). "Gene control of resistance vs. sensitivity to actidione in Saccharomyces." Genetics 42(1): 66-71.

Miyadera, H., K. Shiomi, et al. (2003). "Atpenins, potent and specific inhibitors of mitochondrial complex II (succinate-ubiquinone oxireductase)." Proceedings of the National Academy of Sciences 100(2): 473-477.

Miyamoto, Y., K. Machida, et al. (2002). "Identification of Saccharomyces cerevisiae Isoleucyl-tRNA synthetase as a target of the G1-specific inhibitor reveromycin A." Journal of Biological Chemistry 277(32): 28810-28814. 
Moore, R. N., G. Bigam, et al. (1985). "Biosynthesis of the hypocholesterolemic agent mevinolin by Aspergillus terreus. Determination of the origin of carbon, hydrogen, and oxygen atomes by C-13 NMR and mass spectrometry." Journal of the American Chemical Society 107(12): 3694-3701.

Morris, P. G. and M. N. Fornier (2008). "Microtubule active agents: Beyond the taxane frontier." Clinical Cancer Research 14(22): 7167-7172.

Mukhopadhyay, T., K. Roy, et al. (1987). "Fumifungin, a new antifungal antibiotic from Aspergillus fumigatus fresenius 1863." Journal of Antibiotics 40(7): 1050-1052.

Nagiec, M. M., E. E. Nagiec, et al. (1997). "Sphingolipid synthesis as a target for antifungal drugs." Journal of Biological Chemistry 272(15): 9809-9817.

Nakano, H., M. Hara, et al. (1988). "Paxisterol, a new analgesic sterol without antiinflammation activity from Penicillium." Journal of Antibiotics 41(3): 409410.

Newman, D. J. and G. M. Cragg (2007). "Natural products as sources of new drugs over the last 25 years." Journal of Natural Products 70(3): 461-477.

Niedenthal, R. K., L. Riles, et al. (1996). "Green fluorescent protein as a marker for gene expression and subcellular localization in budding yeast." Yeast 12(8): 773-786.

Nozawa, K. and S. Nakajima (1979). "Studies on fungal products. 6. Isolation of radicicol from Penicillium luteo aurantium, and meleagrin, a new metabolite from Penicillium meleagrinium." Lournal of Natural Products 42(4): 374-377.

Nyren, P. and A. Strid (1989). "The effect of equisetin on energy-linked reactions in Rhodospirillum rubrum chromatophores." Archives of Biochemistry and Biophysics 268(2): 659-666.

Ogita, A., K. Matsumoto, et al. (2007). "Synergistic fungicidal activities of amphotericin B and N-methyl-N "-dodecylguanidine: A constituent of polyol macrolide antibiotic niphimycin." Journal of Antibiotics 60(1): 27-35. 
Okuma, Y., K. Kiguchi, et al. (2003). "Correlation between expression of oncogene products and resistance to anticancer drugs in cultured ovarian cancer cell lines." Human Cell 16(3): 131-139.

Orij, R., J. Postmus, et al. (2009). "In vivo measurement of cytosolic and mitochondrial $\mathrm{pH}$ using a $\mathrm{pH}$-sensitive GFP derivative in Saccharomyces cerevisiae reveals a relation between intracellular $\mathrm{pH}$ and growth." Microbiology 155(1): 268-278.

Pangborn, M. C. (1942). "Isolation and purification of a serologically active phospholipid from beef heart." Journal of Biological Chemistry 143(1): 247256.

Parsons, A., A. Lopez, et al. (2006). "Exploring the mode-of-action of bioactive compounds by chemical-genetic profiling in yeast." Cell 126: 611 - 625.

Parsons, A. B., R. L. Brost, et al. (2004). "Integration of chemical-genetic and genetic interaction data links bioactive compounds to cellular target pathways." Nature Biotechnology 22(1): 62-69.

Patham, B., J. Duffy, et al. (2009). "Post-translational import of protein into the endoplasmic reticulum of a trypanosome: An in vitro system for discovery of anti-trypanosomal chemical entities." Biochemical Journal 419: 507-517.

Pelechano, V., S. Jimeno-Gonzalez, et al. (2009). "Regulon-specific control of transcription elongation across the yeast genome." PLoS Genet 5(8): e1000614.

Perrone, G. G., S.-X. Tan, et al. (2008). "Reactive oxygen species and yeast apoptosis." Biochimica et Biophysica Acta (BBA) - Molecular Cell Research 1783(7): 1354-1368.

Planta, R. J. and W. H. Mager (1998). "The list of cytoplasmic ribosomal proteins of Saccharomyces cerevisiae." Yeast 14(5): 471-477.

Pollard, M. G., K. J. Travers, et al. (1998). "Ero1p: A novel and ubiquitous protein with an essential role in oxidative protein folding in the endoplasmic reticulum." Molecular cell 1(2): 171-182. 
Pozniakovsky, A. I., D. A. Knorre, et al. (2005). "Role of mitochondria in the pheromone- and amiodarone-induced programmed death of yeast." Journal of Cell Biology 168: 257-269.

Przedborski, S., Jackson-Lewis V., Muthane, U., Jiang, H., Ferreira, M., Naini, A.B., Fahn, S. (1993). "Chronic levodopa administration alters cerebral mitochondrial respiratory chain activity." Annals of Neurology 34(5): 715723.

Puig, O., F. Caspary, et al. (2001). "The tandem affinity purification (TAP) method: A general procedure of protein complex purification." Methods 24(3): 218229.

Ramsay, R. R., M. J. Krueger, et al. (1991). "Interaction of 1-methyl-4phenylpyridinium ion $(\mathrm{MPP}+)$ and its analogs with the rotenone/piericidin binding site of NADH dehydrogenase." Journal of Neurochemistry 56(4): 1184-1190.

Rand, J. D. and C. M. Grant (2006). "The thioredoxin system protects ribosomes against stress-induced aggregation." Mol. Biol. Cell 17(1): 387-401.

Rao, N. A., B. Mackler, et al. (1963). "Flavin mononucleotide: Coenzyme of reduced diphosphopyridine nucleotide dehydrogenase." Journal of Biological Chemistry 238(1): 449-\&.

Raskin, I. (1992). "Salicylate, a new plant hormone." Plant Physiology 99(3): 799803.

Ricci, J. E., N. Waterhouse, et al. (2003). "Mitochondrial functions during cell death, a complex (I-V) dilemma." Cell Death Differ 10(5): 488-492.

Rice-Evans, C. A., Gopinathan, V. (1995). "Oxygen toxicity, free radicals and antioxidants in human disease: biochemical implications in atherosclerosis and the problems of premature neonates." Essays Biochem 29: 39-63.

Rigaut, G., A. Shevchenko, et al. (1999). "A generic protein purification method for protein complex characterization and proteome exploration." Nat Biotech 17(10): 1030-1032. 
Rine, J., W. Hansen, et al. (1983). "Targeted selection of recombinant clones through gene dosage effects." Proceedings of the National Academy of Sciences of the United States of America-Biological Sciences 80(22): 6750-6754.

Robinson, N. C., J. Zborowski, et al. (1990). "Cardiolipin-depleted bovine heart cytochrome c oxidase: binding stoichiometry and affinity for cardiolipin derivatives." Biochemistry 29(38): 8962-8969.

Roos, W. P., E. Jost, et al. (2011). "Intrinsic anticancer drug resistance of malignant melanoma cells is abrogated by interferon- $\tilde{A} \ddot{Y}$ and valproic acid." Cancer Research.

Ross-Macdonald, P., P. S. R. Coelho, et al. (1999). "Large-scale analysis of the yeast genome by transposon tagging and gene disruption." Nature 402(6760): 413418.

Royles, B. J. L. (1995). "Naturally-occuring tetramic acids - Structure, isolation, and synthesis." Chemical Reviews 95(6): 1981-2001.

Saisivam, S., D. Bhikshapathi, et al. (2008). "Isolation of borrelidin from Streptomyces californicus - An Indian soil isolate." Indian Journal of Biotechnology 7(3): 349-355.

Salmon, T. B., B. A. Evert, et al. (2004). "Biological consequences of oxidative stressinduced DNA damage in Saccharomyces cerevisiae." Nucleic Acids Research 32(12): 3712-3723.

Schimana, J., H. P. Fiedler, et al. (2000). "Simocyclinones, novel cytostatic angucyclinone antibiotics produced by Streptomyces antibioticus Tu $6040 \mathrm{I}$. Taxonomy, fermentation, isolation and biological activities." Journal of Antibiotics 53(8): 779-787.

Schobert, R. and A. Schlenk (2008). "Tetramic and tetronic acids: An update on new derivatives and biological aspects." Bioorganic \& Medicinal Chemistry 16(8): 4203-4221.

Schreiber, S. L. (2000). "Target-oriented and diversity-oriented organic synthesis in drug discovery." Science 287(5460): 1964-1969. 
Schulte, T. W., S. Akinaga, et al. (1998). "Antibiotic radicicol binds to the Nterminal domain of Hsp90 and shares important biologic activities with geldanamycin." Cell Stress \& Chaperones 3(2): 100-108.

Scott, P. M., Vanwalbe.W, et al. (1971). "Cladosporin, a new antifungal metabolite from Cladosporium-cladosporioides." Journal of Antibiotics 24(11): 747-755.

Sedensky, M. M. and P. G. Morgan (2006). "Mitochondrial respiration and reactive oxygen species in mitochondrial aging mutants." Experimental Gerontology 41(3): 237-245.

Serkova, N. J., Christians, U., Benet, L.Z. (2004). "Biochemical mechanisms of cyclosporin neurotoxicity." Molecular Interventions 4(2): 97-107.

Sevier, C. S., H. Qu, et al. (2007). "Modulation of cellular disulfide-bond formation and the ER redox environment by feedback regulation of Ero1." Cell 129(2): 333-344.

Shannon, P., A. Markiel, et al. (2003). "Cytoscape: A software environment for integrated models of biomolecular interaction networks." Genome Research 13(11): 2498-2504.

Shimizu, T., T. Usui, et al. (2002). "Chemical modification of reveromycin A and its biological activities." Bioorganic \& Medicinal Chemistry Letters 12(23): 33633366.

Sies, H. (1985). "Oxidative stress: Introductory remarks." Oxidative Stress: 147-160.

Simmons, T. L., E. Andrianasolo, et al. (2005). "Marine natural products as anticancer drugs." Molecular Cancer Therapeutics 4(2): 333-342.

Sims, J. W., J. P. Fillmore, et al. (2005). "Equisetin biosynthesis in Fusarium heterosporum." Chemical Communications(2): 186-188.

Singh, B. K., S. S. Bisht, et al. (2006). "An efficient synthesis of tetramic acid derivatives with extended conjugation from L-Ascorbic Acid." Beilstein Journal of Organic Chemistry 2.

Singh, K., S. Sun, et al. (1979). "Rapamycin (AY-22,989), a new antifungal antibiotic. 4. Mechanism of action." Journal of Antibiotics 32(6): 630-645. 
Singh, M. P., J. Zaccardi, et al. (1998). "LL-49F233 alpha, a novel antibiotic produced by an unknown fungus: Biological and mechanistic activities." Journal of Antibiotics 51(12): 1109-1112.

Singh, S. B., D. L. Zink, et al. (1998). "Equisetin and a novel opposite stereochemical homolog phomasetin, two fungal metabolites as inhibitors of HIV-1 integrase." Tetrahedron Letters 39(16): 2243-2246.

Singh, S. B., D. L. Zink, et al. (1998). "Kampanols: Novel ras farnesyl-protein transferase inhibitors from Stachybotrys kampalensis." Bioorganic \& Medicinal Chemistry Letters 8(16): 2071-2076.

Skulachev, V. P. (1996). "Why are mitochondria involved in apoptosis? Permeability transition pores and apoptosis as selective mechanisms to eliminate superoxide-producing mitochondria and cell." FEBS Letters 397(1): 7-10.

Sobell, H. M. (1985). "Actinomycin and DNA transcription." Proceedings of the National Academy of Sciences of the United States of America 82(16): 53285331.

Sopko, R., D. Huang, et al. (2006). "Mapping pathways and phenotypes by systematic gene overexpression." Mol Cell 21: 319 - 330.

Starkov, A. A. and G. Fiskum (2001). "Myxothiazol induces $\mathrm{H}_{2} \mathrm{O}_{2}$ production from mitochondrial respiratory chain." Biochemical and Biophysical Research Communications 281(3): 645-650.

Stearns, T., M. A. Hoyt, et al. (1990). "Yeast mutants sensitive to antimicrotubule drugs define three genes that affect microtubule function." Genetics 124(2): 251-262.

Stevens, B. (1981). Mitochondrial structure. Cold Spring Harbor, NY, Cold Spring Harbor Laboratory Press.

Stewart, M., R. J. Capon, et al. (2005). "Calbistrin E and two other new metabolites from an Australian isolate of Penicillium striatisporum." Journal of Natural Products 68(4): 581-584. 
Stocklein, W. and W. Piepersberg (1980). "Altered ribosomal-protein L29 in a cycloheximide-resistant strain of Saccharomyces cerevisiae." Current Genetics 1(3): 177-183.

Stoessl, A., R. J. Cole, et al. (1989). "Some biological properties of traversianal, a strongly molluscicidal diterpenoid aldehyde from Cercospora traversiana." Mycopathologia 106(1): 41-46.

Sturgeon, C. M., D. Kemmer, et al. (2006). "Yeast as a tool to uncover the cellular targets of drugs." Biotechnology Journal 1(3): 289-298.

Sugie, Y., e. al, et al. (2002). "CJ-21,058, a new SecA inhibitor isolated from a fungus." ChemInform 33(27): no-no.

Sutton, C. A., M. Ares, et al. (1978). "Cycloheximide resistance can be mediated through either ribosomal-subunit." Proceedings of the National Academy of Sciences of the United States of America 75(7): 3158-3162.

Tan, S.-X., M. Teo, et al. (2009). "Cu, Zn superoxide dismutase and NADP(H) homeostasis are required for tolerance of endoplasmic reticulum stress in Saccharomyces cerevisiae." Mol. Biol. Cell 20(5): 1493-1508.

Tatsuta, K., M. Itoh, et al. (1997). "The first total synthesis of calbistrin A, a microbial product possessing multiple bioactivities." Tetrahedron Letters 38(4): 583-586.

Temple, M., G. Perrone, et al. (2005). "Complex cellular responses to reactive oxygen species." Trends Cell Biol 15: 319 - 326.

Terada, H. (1990). "Uncouplers of oxidative phosphorylation." Environmental Health Perspectives 87: 213-218.

Thierbach, G. and G. Michaelis (1982). "Mitochondrial and nuclear myxothiazol resistance in Saccharomyces cerevisiae." Mol Gen Genet 186(4): 501-6.

Thierbach, G. and H. Reichenbach (1981). "Myxothiazol, a new inhibitor of the cytochrome b-c1 segment of th respiratory chain." Biochim Biophys Acta 638(2): 282-9. 
Thomas, J. H., N. F. Neff, et al. (1985). "Isolation and characterisation of mutations in the beta-tubulin gene of Saccharomyces cerevisiae." Genetics 111(4): 715734.

Thorpe, G., C. Fong, et al. (2004). "Cells have distinct mechanisms to maintain protection against different reactive oxygen species: oxidative-stress-response genes." Proc Natl Acad Sci USA 101: 6564 - 6569.

Tong, A. H. Y., M. Evangelista, et al. (2001). "Systematic genetic analysis with ordered arrays of yeast deletion mutants." Science 294(5550): 2364-2368.

Turrens, J. F. (2003). "Mitochondrial formation of reactive oxygen species." The Journal of Physiology 552(2): 335-344.

Turrens, J. F., A. Alexandre, et al. (1985). "Ubisemiquinone is the electron donor for superoxide formation by complex III of heart mitochondria." $\underline{\text { Archives of }}$ Biochemistry and Biophysics 237(2): 408-414.

Usui, T., G. Marriott, et al. (1999). "Protein phosphatase 2A inhibitors, phoslactomycins. Effects on the cytoskeleton in NIH/3T3 cells." Journal of Biochemistry 125(5): 960-965.

Usuki, Y., K. Matsumoto, et al. (2006). "Structure-activity relationship studies on niphimycin, a guanidylpolyol macrolide antibiotic. Part 1: The role of the Nmethyl-N "-alkylguanidinium moiety." Bioorganic \& Medicinal Chemistry Letters 16(6): 1553-1556.

Valli, M., M. Sauer, et al. (2005). "Intracellular pH distribution in Saccharomyces cerevisiae cell populations, analyzed by flow cytometry." Appl. Environ. Microbiol. 71(3): 1515-1521.

Vane, J. (1994). "Towards a better aspirin." Nature 367(6460): 215-216.

Vesonder, R. F., L. W. Tjarks, et al. (1979). "Equisetin, an antibiotic from Fusarium equiseti NRRL-5537, identified as a derivative of N-methyl-2,4-pyrollidone." Journal of Antibiotics 32(7): 759-761. 
Vezina, C., A. Kudelski, et al. (1975). "Rapamycin (AY-22,989), a new antifungal antibiotic. 1. Taxonoomy of producing Streptomycete and isolation of active principle." Journal of Antibiotics 28(10): 721-726.

Wakabaya.K and N. Gunge (1970). "Extrachromosomal inheritance of oligomycin resistance in yeast." FEBS Letters 6(4): 302-\&.

Walsh, C. (2000). "Molecular mechanisms that confer antibacterial drug resistance." Nature 406(6797): 775-781.

Wang, H. and J. A. Joseph (1999). "Quantifying cellular oxidative stress by dichlorofluorescein assay using microplate reader." Free Radical Biology and Medicine 27(5-6): 612-616.

Wani, M. C., H. L. Taylor, et al. (1971). "Plant antitumor agents .6. Isolation and structure of taxol, a novel antileukemic and antitumor agent from Taxus brevifolia." Journal of the American Chemical Society 93(9): 2325-2327.

Wen, K.-K. and P. A. Rubenstein (2009). "Differential regulation of actin polymerization and structure by yeast formin isoforms." Journal of Biological Chemistry 284(25): 16776-16783.

Wheeler, M. H., R. D. Stipanovic, et al. (1999). "Phytotoxicity of equisetin and epiequisetin isolated from Fusarium equiseti and F. pallidoroseum." Mycological Research 103: 967-973.

Wheeler, W. J. (1977). "Cephalosporins and other beta-lactam antibiotics." Lloydiathe Journal of Natural Products 40(6): 519-542.

White, T. C., K. A. Marr, et al. (1998). "Clinical, cellular, and molecular factors that contribute to antifungal drug resistance." Clin. Microbiol. Rev. 11(2): 382402.

Wilkie, D. and B. K. Lee (1965). "Genetic analysis of actidione resistance in Saccharomyces cerevisiae." Genetical Research 6(1): 130-\&.

Winzeler, E. A., D. D. Shoemaker, et al. (1999). "Functional characterization of the $S$. cerevisiae genome by gene deletion and parallel analysis." Science 285(5429): 901-906. 
Winzeler, E. A., D. D. Shoemaker, et al. (1999). "Functional characterization of the $S$. cerevisiae genome by gene deletion and parallel analysis." Science 285(5429): 901-906.

Wissing, S., P. Ludovico, et al. (2004). "An AIF orthologue regulates apoptosis in yeast." The Journal of Cell Biology 166(7): 969-974.

Woo, J. T. (2007). "Reveromycin A, a novel agent for osteoporosis inhibits bone resorption by inducing apoptosis specifically in osteoclasts." Lournal of Pharmacological Sciences 103: 27P-27P.

Woo, J. T., M. Kato, et al. (1999). "Reveromycin A induces osteoclast apoptosis and inhibits bone resorption." Journal of Bone and Mineral Research 14: SA207.

Wright, R. M., S. L. Simpson, et al. (1995). "Oxygen regulation of the cytochrome c oxidase subunit VI gene, COX6, in Saccharomyces cerevisiae." Biochemical and Biophysical Research Communications 216(2): 676-685.

Xiao, W., B. L. Chow, et al. (1998). "Mms4, a putative transcriptional (co)activator, protects Saccharomyces cerevisiae cells from endogenous and environmental DNA damage." Molecular \& General Genetics 257(6): 614-623.

Yin, M.-J., Y. Yamamoto, et al. (1998). "The anti-inflammatory agents aspirin and salicylate inhibit the activity of IkappaB kinase-beta." Nature 396(6706): 7780.

Yu, L., A. Lopez, et al. (2008). "Chemical-genetic profiling of imidazo[1,2a]pyridines and -pyrimidines reveals target pathways conserved between yeast and human cells." Plos Genetics 4(11).

Yuichi S., H. S., Koji Y. (2004). "IPC synthase as a useful target for antifungal drugs." Current Drug Targets - Infectious Disorders 4: 311-322.

Yuki, K., M. Shindo, et al. (2001). "Enantioselective total synthesis of (-)-equisetin using a Me3Al-mediated intramolecular Diels-Alder reaction." Tetrahedron Letters 42(13): 2517-2519. 
Zhang, H., C. Gajate, et al. (2007). "Mitochondrial-derived ROS in edelfosineinduced apoptosis in yeasts and tumor cells." Acta Pharmacol Sin 28(6): 888894.

Zhang, L., L. Yu, et al. (1998). "Generation of superoxide anion by succinatecytochrome $c$ reductase from bovine heart mitochondria." Journal of Biological Chemistry 273(51): 33972-33976.

Zhang, Y., H. Qi, et al. (2007). "The role of autophagy in mitochondria maintenance: Characterization of mitochondrial functions in autophagy-deficient $S$. cerevisiae strains." Autophagy 3(4): 337-346.

Zhao, R., M. Davey, et al. (2005). "Navigating the Chaperone Network: An Integrative Map of Physical and Genetic Interactions Mediated by the Hsp90 Chaperone." Cell 120(5): 715-727.

Zheng, C. J., H. E. Yu, et al. (2008). "Viridicatumtoxin B, a new anti-MRSA agent from Penicillum sp FR11." Journal of Antibiotics 61(10): 633-637. 
